\title{
Enhancing AI-based Game Playing Using Adaptive Data Structures
}

\author{
By \\ Spencer Polk \\ A thesis submitted to \\ the Faculty of Graduate Studies and Research \\ in partial fulfilment of \\ the requirements for the degree of \\ Doctor of Philosophy \\ Ottawa-Carleton Institute for Computer Science \\ School of Computer Science \\ Carleton University \\ Ottawa, Ontario
}

June 2016

(C) Copyright

2016, Spencer Polk 
The undersigned hereby recommend to the Faculty of Graduate Studies and Research acceptance of the thesis,

\section{Enhancing AI-based Game Playing}

\section{Using Adaptive Data Structures}

submitted by

Spencer Polk

Dr. Douglas Howe

(Director, School of Computer Science)

Dr. B. John Oommen

(Thesis Supervisor)

(External Examiner)

Carleton University

June 2016 


\begin{abstract}
The design and analysis of strategies for playing strategic board games, is a core area of Artificial Intelligence (AI), that has been studied extensively since the inception of the field. However, while two-player board games are very well known, comparatively little research has been done on Multi-Player games, where the number of self-interested, competing players is greater than two. Furthermore, known strategies for multi-player games have difficulties performing on a level of sophistication comparable to their two-player counterparts.

When progress in a field is stymied, promising approaches can be discovered through drawing inspiration from other, completely unrelated areas of Computer Science. The premise of this thesis is the hypothesis that game playing in general, and the problem of multi-player games in particular, can benefit from efficient ranking mechanisms, for moves, board positions, or even players, in the multi-player scenario. The research done in this work confirms the hypothesis. Indeed, we have discovered that this information can be applied to improve game tree pruning, through the concept of move ordering, and within other possibilities. In this thesis, we observe that the formerly-unrelated field of Adaptive Data Structures (ADSs), which provide mechanisms by which a data structure can reorganize itself internally in response to queries, can provide a natural ranking mechanism. The primary motivation of this thesis is to demonstrate that the low-cost ADS-based data structures can provide this ranking mechanism to game-playing engines, and furthermore generate statistically significant improvements to their efficiency.

In this work, we will conclusively prove that ADS-based techniques are able to enhance existing multi-player game playing strategies, and perform competitively with state-of-the-art two-player techniques, as well. We demonstrate, through two general-use, domain independent move ordering heuristics, the Threat-ADS heuristic
\end{abstract}


for multi-player games, and the History-ADS heuristic for both two-player and multiplayer games, that ADSs are, indeed, capable of achieving this improvement. We present an examination of their performance in a very wide range of game models, configurations, and by employing differing ADSs and refinements. We thus conclusively demonstrate that ADSs are able to achieve strong performance, in game playing engines, in the vast majority of cases. Our work in this thesis provides not only these domain-independent, formal move ordering heuristics, but furthermore serves as a strong example for future investigation into combinations between the fields of ADSs and game playing. 
Dedicated with all my love to

my parents and grandparents

Nancy, Jeff, Elaine, and Leo 


\section{Acknowledgements}

Above all, I express my utmost gratitude to my thesis advisor, Professor John Oommen, for his continuing patience, support, and kindness during this part of my voyage through life. Without his encouragement throughout this process, I would be looking back on what I had done with doubt, and forward to my future in academia and beyond, with fear. Through his generous guidance and advice, the experience has rather been one of exploration and discovery. I would also like to sincerely thank him for providing me with the opportunity to augment my studies through lecturing. I will be grateful to him for all he has done for me for the rest of my life.

I would like to thank the members of the thesis proposal committee, for their feedback on earlier drafts of this work. I am grateful for their experienced advice, which has helped guide me in my research and the design of my experiments, as well as the formulation of this final work.

I would also like to express my thanks to my co-workers and and supervisors at Purple Forge for allowing me unprecedented leeway in shaping my work schedule to fit my academic goals. I would like to especially thank the founder and CEO, Brian Hurley, who not only accommodated my unpredictable work/school schedule, but also provided me with valuable advice on the commercial applications of my research in the industry, as well as an opportunity to experience "real-world" AI systems.

I am grateful to the School of Computer Science, and its administrative staff, and faculty, for all of their support throughout these long years. In particular, I am thankful to Dr. Douglas Howe, for providing me with the opportunity to teach a course during my time as a graduate student. 
Finally, I thank my family for encouraging and supporting me well beyond anything I could have asked for through this process, and for showing me infinite patience when it concerned my tendency to ramble on about my research. I am sincerely grateful for being shown such love and patience. Thank you all from the bottom of my heart! 


\section{Contents}

1 Introduction 1

1.1 Problem Overview and Motivation . . . . . . . . . . . . . 2

1.2 Problem Approach . . . . . . . . . . . . . . . . . . . . . 4

1.2.1 Verification of the Approach . . . . . . . . . . . 7

1.2.2 Complexity of the Verification . . . . . . . . . . . 7

1.3 Contributions of the Thesis . . . . . . . . . . . . . . 9

1.4 Structure of the Thesis . . . . . . . . . . . . . . . . 10

2 Survey of the Field $\quad 13$

2.1 Chapter Overview . . . . . . . . . . . . . . . . . 13

2.2 Families of Games . . . . . . . . . . . . . . . . . . . . . . . . . . 14

2.3 Game Playing: Overview . . . . . . . . . . . . . . . . . . 17

2.3.1 Historical Overview . . . . . . . . . . . . . . . 17

2.3.2 Game Trees . . . . . . . . . . . . . . . . 18 
2.3 .3 Branch and Bound . . . . . . . . . . . . . . . . . . . 19

2.4 Two-Player Game Playing . . . . . . . . . . . . . . . . 20

2.4.1 The Mini-Max Algorithm _. . . . . . . . . . . 20

2.4 .2 Alpha-Beta Search . . . . . . . . . . . . . . . . . . 21

2.4 .3 Move Ordering . . . . . . . . . . . . . . . . . . . 24

2.4.3.1 Killer Moves and the History Heuristic . . . . . . . 25

2.4.4 Other Alpha-Beta Related Techniques . . . . . . . . . . 27

2.4.4.1 Transposition Tables . . . . . . . . . . . . 27

2.4.4.2 The Horizon Effect and Quiescence Search . . . . . 29

2.4.4.3 Stochastic Improvements . . . . . . . . . . . . . . 29

2.4.4.4 Handling Imperfect Information . . . . . . . . . . 30

2.4.5 Non-Alpha-Beta Strategies for Two-Player Games . . . . . . . 31

2.5 Multi-Player Game Playing . . . . . . . . . . . . . . . . 33

2.5.1 Challenges of Multi-Player Games . . . . . . . . . . . . . . 33

2.5.2 The Paranoid Algorithm . . . . . . . . . . . . . . . . 34

2.5.3 The Max-N Algorithm . . . . . . . . . . . . . . . . . 36

2.5.3.1 Extensions to Max-N . . . . . . . . . . . 38

2.5.4 The Best-Reply Search . . . . . . . . . . . . . . . . . . . . . . 39

2.6 Stochastic Methods . . . . . . . . . . . . . . . . . . . . . . 41

2.6.1 Monte Carlo Tree Search . . . . . . . . . . . . . . . . . . 42 
2.6.2 The UCT Algorithm . . . . . . . . . . . . . . . . . . 43

2.7 The Improving Agent: Adaptive Data Structures $\ldots \ldots \ldots \ldots \ldots$

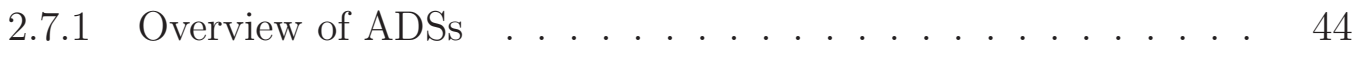

$2.7 .2 \quad$ List-Based ADSs . . . . . . . . . . . . . . . . . . . 46

2.7.2.1 Move-to-Front . . . . . . . . . . . . 46

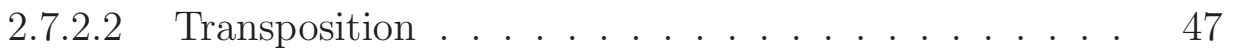

2.7.2.3 Move-Ahead-k . . . . . . . . . . . . . . . 48

$2.7 .2 .4 \quad \mathrm{POS}(\mathrm{k}) \ldots \ldots \ldots \ldots \ldots$

2.7.2.5 Stochastic Variants . . . . . . . . . . . . 50

$2.7 .3 \quad$ Tree-Based ADSs . . . . . . . . . . . . . . . . . . . 51

2.8 Chapter Summary . . . . . . . . . . . . . . . . . . . . . . . 53

3 Improving the Best-Reply Search using ADS 54

3.1 Chapter Overview . . . . . . . . . . . . . . . . . . . . 54

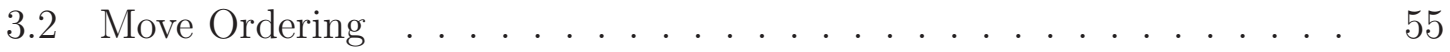

3.2 .1 Benefits of Move Ordering . . . . . . . . . . . 55

3.2 .2 Examples of Move Ordering . . . . . . . . . . . 56

3.3 Opponent Threat Level . . . . . . . . . . . . . . . . . . . . . . 58

3.3.1 Properties of Opponent Threat . . . . . . . . . . . 59

3.3.2 Opponent Threat and Move Ordering . . . . . . . . . . 60 
3.4 Managing Opponent Threat Level Using an ADS . . . . . . . . . . . 62

3.5 The Threat-ADS Heuristic . . . . . . . . . . . . . . 67

3.5.1 Developing the Threat-ADS Heuristic . . . . . . . . . . . . . 67

3.5.2 Salient Features of the Threat-ADS Heuristic . . . . . . . . . 69

3.6 Experimental Model . . . . . . . . . . . . . . . . . . . 71

3.7 Prima Facie Experiments . . . . . . . . . . . . . . . . . . 73

3.8 Variation on the Number of Players . . . . . . . . . . . . . . . 75

3.8.1 Results ..................... 76

3.9 Variation on the Starting State of the Game . . . . . . . . . . . . 78

3.9.1 Results ....................... . . 79

3.10 Evaluation of Results . . . . . . . . . . . . . . . . . . . . 82

3.11 Chapter Conclusions . . . . . . . . . . . . . . . 85

4 Improvements to the Threat-ADS Heuristic $\quad 86$

4.1 Chapter Overview . . . . . . . . . . . . . . 86

4.2 Open Questions . . . . . . . . . . . . . . . . 87

4.3 Threat-ADS' Behaviour Using Different ADSs . . . . . . . . . . 88

4.4 Ergodic versus Absorbing ADSs . . . . . . . . . . . . . . . . 90

4.5 Investigating the Threat-ADS' Performance at Different Ply Levels 92

4.6 Experimental Model . . . . . . . . . . . . . . . . 93 
4.7 Initial Board Position Experiments . . . . . . . . . . . . . 95

4.8 Midgame Starting Position Experiments . . . . . . . . . . . . . 99

4.9 Discussion . . . . . . . . . . . . . . . . . . . . . . 102

4.10 Chapter Conclusions . . . . . . . . . . . . . . 105

5 History-Based Move Ordering using ADSs 106

5.1 Chapter Overview . . . . . . . . . . . . . . . 106

5.2 Motivation ........................... 107

5.3 Move Ordering Using Move History . . . . . . . . . . . . . . . . . . . 108

5.4 Managing Move History Using an ADS . . . . . . . . . . . . . . . . . 109

5.5 The History-ADS Heuristic . . . . . . . . . . . . . . . . . . . . . 112

5.5.1 Developing the History-ADS Heuristic . . . . . . . . . . . . . 112

5.5.2 Salient Features of the History-ADS Heuristic . . . . . . . . . 113

5.6 Experimental Model . . . . . . . . . . . . . . . . 115

5.7 Results for Two-Player Games . . . . . . . . . . . . . . . . . . . 118

5.8 Results for Multi-Player Games . . . . . . . . . . . . . . . . . 121

5.9 Discussion . . . . . . . . . . . . . . . . . . . . 123

5.10 Chapter Conclusions . . . . . . . . . . . . . . 126

6 Refinements of the History-ADS Heuristic 127

6.1 Chapter Overview . . . . . . . . . . . . . . . . . 127 
6.2 Open Questions . . . . . . . . . . . . . . . . . . 128

6.3 Restricting the Length of the ADS . . . . . . . . . . . . . . 129

6.3.1 Strategy and Technique . . . . . . . . . . . . . . 129

6.3.2 Experimental Model . . . . . . . . . . . . . . . . 130

6.3.3 Results for Bounded ADSs . . . . . . . . . . . . . . . . 132

6.4 Multi-level ADSs . . . . . . . . . . . . . . . . . . . . 138

6.4.1 Strategy and Technique . . . . . . . . . . . . . . . 138

6.4.2 Experimental Model . . . . . . . . . . . . . . . 140

6.4.3 Experimental Results for Multi-Level ADSs . . . . . . . . . . 140

6.5 Discussion . . . . . . . . . . . . . . . . . . . . 143

6.6 Chapter Conclusions . . . . . . . . . . . . . . . . . 145

7 History-ADS Compared to Existing Move-Ordering Heuristics 147

7.1 Chapter Overview . . . . . . . . . . . . . . . . 147

7.2 Motivation . . . . . . . . . . . . . . . . . 148

7.3 ADS-based implementation of the History Heuristic . . . . . . . . . . 149

7.4 Experimental Model . . . . . . . . . . . . . . . . . . 152

7.5 Results for the History Heuristic . . . . . . . . . . . . . . . . . . 153

7.6 Implementing the Killer Moves heuristic in the context of the HistoryADS Heuristic . . . . . . . . . . . . . . . . . . . 157

7.7 Experimental Model . . . . . . . . . . . . . . . . 159 
7.8 Results for Killer Moves . . . . . . . . . . . . . . . . . . . . . 160

7.9 Discussion . . . . . . . . . . . . . . . . 163

7.10 Chapter Conclusions . . . . . . . . . . . . . 166

8 Summary, Contributions, and Future Work 167

8.1 Summary by Chapters . . . . . . . . . . . . . . . 167

8.2 Contributions of the Thesis . . . . . . . . . . . . 170

8.3 Limitations and Future Work . . . . . . . . . . . . . . . . . 172

$\begin{array}{ll}\text { A Appendix: Game Models } & 175\end{array}$

A.1 Overview . . . . . . . . . . . . . . . . 175

A.2 The Virus Game . . . . . . . . . . . . . . . . . 176

A.3 Focus. . . . . . . . . . . . . . . . . . . . 181

A.4 Chinese Checkers . . . . . . . . . . . . . . . . . . 185

A.5 Relaxed Checkers . . . . . . . . . . . . . . . . . . . . . . 189

A.6 Othello. . . . . . . . . . . . . . . . . . . . . . . . 192

A.7 Summary . . . . . . . . . . . . . . . . . . . . 194

$\begin{array}{ll}\text { Bibliography } & 195\end{array}$ 


\section{List of Tables}

3.1 Results of the Threat-ADS for the various Game Models . . . . . . . 74

3.2 Results by Game Model - Three Players . . . . . . . . . . . . . . 76

3.3 Results by Game Model - Four Players . . . . . . . . . . . . . . . . . 77

3.4 Results by Game Model - Five Players . . . . . . . . . . . . . . . . . 78

3.5 Results by Game Model - Six Players . . . . . . . . . . . . . . . . . . 78

3.6 Results by Game Model - Three Players - Midgame Start . . . . . . . 80

3.7 Results by Game Model - Four Players - Midgame Start . . . . . . . 80

3.8 Results by Game Model - Five Players - Midgame Start . . . . . . . . 81

3.9 Results by Game Model - Six Players - Midgame Start . . . . . . . . 81

4.1 Results for the 4-ply Virus Game when the measurement started from an initial game position. . . . . . . . . . . . . . 96

4.2 Results for the 6-ply Virus Game when the measurement started from an initial game position. . . . . . . . . . . . . . . 96

4.3 Results for the 4-player Chinese Checkers scenario when the measurement started from an initial game position. . . . . . . . . . . . . . 97 
4.4 Results for the 6-player Chinese Checkers scenario when the measurement started from an initial game position. . . . . . . . . . . . 98

4.5 Results for Focus when the measurement started from an initial game position. . . . . . . . . . . . . . . . . . 98

4.6 Average Tree Size for the 4-ply Virus Game when the measurement started from a midgame game position. . . . . . . . . . . . . . . 99

4.7 Average Tree Size for the 6-ply Virus Game when the measurement started from a midgame game position. . . . . . . . . . . 100

4.8 Average Tree Size for the 4-player Chinese Checkers scenario when the measurement started from a midgame game position. . . . . . . . . . 100

4.9 Average Tree Size for the 6-player Chinese Checkers scenario when the measurement started from a midgame game position. . . . . . . . . . 101

4.10 Average Tree Size for Focus when the measurement started from a midgame game position, with a five-turn setup. . . . . . . . . . . 102

4.11 Average Tree Size for Focus when the measurement started from a midgame game position, with a three-turn setup. . . . . . . . . . 102

5.1 Results of applying the History-ADS heuristic for Othello in various configurations. . . . . . . . . . . . . . . . . . . . . . 119

5.2 Results of applying the History-ADS heuristic to Relaxed Checkers in various configurations. . . . . . . . . . . . . . . 120

5.3 Results of applying the History-ADS heuristic to two-player Focus in initial and midgame states. . . . . . . . . . . . . . . . . . . 121

5.4 Results of applying the History-ADS heuristic to the Virus Game in various configurations. . . . . . . . . . . . . . 122 
5.5 Results of applying the History-ADS heuristic to multi-player Focus in initial and midgame cases. . . . . . . . . . . . . . . . . 123

5.6 Results of applying the History-ADS heuristic to Chinese Checkers in various configurations. . . . . . . . . . . . . . . . 124

6.1 Results of applying the History-ADS heuristic to Othello with a varying maximum length on the ADS. . . . . . . . . . . . . . . . . 133

6.2 Results of applying the History-ADS heuristic to Relaxed Checkers with a varying maximum length on the ADS. . . . . . . . . . . . . . 134

6.3 Results of applying the History-ADS heuristic to Focus with a varying maximum length on the ADS. . . . . . . . . . . . . . . . 135

6.4 Results of applying the History-ADS heuristic to the Virus Game with a varying maximum length on the ADS. . . . . . . . . . . . . 136

6.5 Results of applying the History-ADS heuristic to Chinese Checkers with a varying maximum length on the ADS. . . . . . . . . . . . 137

6.6 Results of applying the History-ADS heuristic to Othello with multilevel ADSs. . . . . . . . . . . . . . . . . . . . . . . . . . 141

6.7 Results of applying the History-ADS heuristic to Relaxed Checkers with multi-level ADSs. . . . . . . . . . . . . . . . . . . . . . 142

6.8 Results of applying the History-ADS heuristic to the Virus Game with multi-level ADSs. . . . . . . . . . . . . . . . . . . . . . . . 143

7.1 Results comparing the History-ADS, the HH-ADS, and the History Heuristic in Othello.

7.2 Results comparing the History-ADS, the HH-ADS, and the History Heuristic in Relaxed Checkers. . . . . . . . . . . . . . . . . . . . . . 155 
7.3 Results comparing the History-ADS, the HH-ADS, and the History Heuristic in Focus. . . . . . . . . . . . . . . . . 156

7.4 Results comparing the History-ADS, the HH-ADS, and the History Heuristic in the Virus Game. . . . . . . . . . . . . . . . . . . 157

7.5 Results comparing the History-ADS, the HH-ADS, and the History Heuristic in Chinese Checkers. . . . . . . . . . . . . . . . . . 158

7.6 Results comparing the History-ADS and Killer Moves in Othello. . . 161

7.7 Results comparing the History-ADS and Killer Moves in Relaxed Checkers. . . . . . . . . . . . . . . . . . . . 162

7.8 Results comparing the History-ADS and Killer Moves in Focus. . . . 163

7.9 Results comparing the History-ADS and Killer Moves in the Virus Game.164

7.10 Results comparing the History-ADS and Killer Moves in Chinese Check-

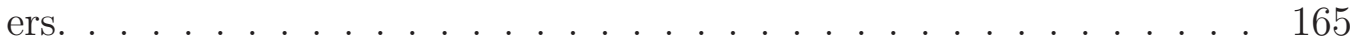




\section{List of Figures}

2.1 Complete game tree for the two-player five-stick Substraction Game .

2.2 A sample Mini-Max tree. Nodes that are tinted red are Max nodes, and nodes that are tinted blue are Min nodes. . . . . . . . . . . . . .

2.3 Sample Paranoid Tree in which the red nodes are MAX nodes, and the blue nodes are MIN nodes. . . . . . . . . . . . . . . . . . . . 35

2.4 Sample Max-N Tree in which each colour represents a different player. 37

2.5 The operation of a single level of the Best-Reply Search. . . . . . . . 40

2.6 Execution of the Move-to-Front update mechanism for the singly-linked list $\mathrm{ADS}$ when the order of the elements accessed is $C, A, B \ldots 47$

2.7 Execution of the Transposition update rule for the singly-linked list where the order of accesses is $C, C, D$. . . . . . . . . . . . . 49

3.1 An example of alpha cutoff when the move ordering is poor. . . . . . 57

3.2 An example of alpha cutoff when the move ordering is planned efficiently. 58

3.3 An example layout of the Virus Game with a threatening opponent. . 60

3.4 The subtree associated with the BRS without considering the various opponents' threat levels. . . . . . . . . . . . . . . . . . . . . . . 
3.5 The subtree associated with the BRS when it is guided by the threat levels of the opponents. . . . . . . . . . . . . . . 63

3.6 An instance of the Focus game showcasing opponent threat levels. . . 64

3.7 A demonstration of how an ADS can be used to learn relative opponent threat levels over time (P1: Red, P2: Blue, P3: Green, P4: Yellow). . 66

3.8 A demonstration of how the Threat-ADS heuristic operates over time.

4.1 A demonstration of the Threat-ADS heuristic updating using a Moveto-Front ADS, obtained by moving player P3 to the front of the list. .

4.2 A demonstration of the Threat-ADS heuristic updating using a Transposition ADS, obtained by moving player P3 one step closer to the front of the list. . . . . . . . . . . . . . . . . . . . . . . . . . . 90

4.3 An example of two players threatening each other in the Virus Game. 91

4.4 An example of how the relative threat levels of opponents can change with time. . . . . . . . . . . . . . . . . . . .

5.1 An example of an alpha-beta search without including move ordering that is based on move history. . . . . . . . . . . . . . . . . . . . . . 109

5.2 An example of an alpha-beta search augmented with move ordering that is based on move history. . . . . . . . . . . . . . . . . . . . . 110

5.3 A demonstration of how an ADS can be used to manage move history over time. . . . . . . . . . . . . . . . . . . . 111

6.1 An example of a History-ADS's list sequence updating over several queries, with a maximum length of 5. . . . . . . . . . . . 130 
6.2 An example of the utility of multiple ADSs in the context of the History-ADS heuristic. . . . . . . . . . . . . . . . 139

7.1 A demonstration of how our approximation of the History Heuristic using an ADS updates the list of available moves. . . . . . . . . . . . 151

7.2 A demonstration of how the Killer Moves technique can be implemented using an ADS. . . . . . . . . . . . . . . . . . 159

A.1 The starting position for the Virus Game. . . . . . . . . . . . . 177

A.2 A possible move for the red player in the Virus Game. . . . . . . . 178

A.3 A comparison between a top-heavy (unbalanced) virulence and a balanced virulence. . . . . . . . . . . . . . . . . . . . 180

A.4 A set of virulences that are considered to be "isomorphic" to each other.181

A.5 The two, three, and four player starting positions for Focus. . . . . . 182

A.6 Possible moves for the grey player in Focus, playing against white. . . 183

A.7 The two, three, four, and six player starting positions for Chinese Checkers. . . . . . . . . . . . . . . . . 186

A.8 A series of jumping moves by the purple player in Chinese Checkers. . 187

A.9 The starting position for the game Checkers. . . . . . . . . . . . 190

A.10 The starting position for the game Othello. . . . . . . . . . . . . . 193

A.11 An example of a valid move in Othello. . . . . . . . . . . . . . . . . . 194 


\section{Chapter 1}

\section{Introduction}

In the broad spectrum of problems addressed by the field of Artificial Intelligence (AI), a particularly common and recurring theme is the need for executing a "search", within an explosively large space. Indeed, we see this problem emerge under a vast number of applications, including data mining, text parsing, and managing network traffic. Traditional search algorithms, such as the Depth-First and Breadth-First searches, are insufficient to manage explosively large search spaces in an acceptable amount of time, and thus, the field of AI has introduced a range of techniques, particularly Branch and Bound strategies, such as the $\mathrm{A}^{*}$ search [48].

In order for strategies, such as the $\mathrm{A}^{*}$ search, to perform expediently, it is necessary to explore candidate solutions in an intelligent order, so that strong constraints can be enforced so as to effectively prune the search space. This can be accomplished through a range of heuristic methods, which achieve an intelligent ranking of candidate solutions, at each level of the expanding solution space. Therefore, a method to improve the performance of intelligent search techniques is to provide stronger ranking mechanisms for these candidates.

A particularly challenging search domain, where the issue of ranking is a canonical 
one in the context of available options, is found in the context of playing an adversarial board game. In this situation, we must deal with an explosively large search space, considering the number of possible moves available to every player. This is further augmented by the presence of opponents who work against our objectives, and introduce a level of uncertainty that any search strategy must account for.

We observe that a formerly-unrelated field of Computer Science, namely, that of Adaptive Data Structures (ADSs), also seeks to solve the problem of intelligent ranking, in the context of the access frequencies of objects within a data structure. In this thesis, our aim is to employ the ranking properties of ADSs, not to merely rank objects in a data structure based on their access probabilities, but rather as a mechanism to intelligently, and efficiently, rank objects in the context of a game's search tree. By employing techniques from the field of ADSs, we will provide novel and powerful strategies for this complex domain, and conclusively demonstrate that they have a positive impact on the efficiency of the game's search tree.

\subsection{Problem Overview and Motivation}

The problem of achieving robust game playing, against an intelligent opponent, is a canonical one in the field of AI. It has seen a great deal of emphasis in the literature surrounding the field, from its inception to the modern day $[24,48,51,54,58]$. The importance of game playing to the field of AI stretches beyond its extensive body of research, as advances in game playing receive significant media attention and become a focal point for the discipline in the public eye. One has to but consider the news coverage surrounding Deep Blue's defeat of Kasparov in the '90s, Watson's victory on the game show "Jeopardy", or the very recent victory of Google's AlphaGo over Lee Sedol, to see how integral to the public's view of AI, the sub-field of game playing is $[30,5,59]$.

The dominant techniques within game playing have been those based on the work done by von Neumann, and elaborated upon by Turing and Shannon [58]. Typically 
referred to as the Mini-Max or Maximin strategy, this technique dictates how a game "tree" may be explored in a logical manner, assuming that the player in question is interacting with an intelligent, adversarial opponent. Briefly, the algorithm operates by exploring the tree in a depth-first manner, and assigning to each node in the tree a value for the "thinking" AI player (hereafter referred to as the perspective player). Further, during the analysis, it assumes that it confronts an equally-intelligent opponent who seeks to hamper his ${ }^{1}$ position. The specification of this algorithm initially targeted the traditional board game Chess, and Chess playing remains a very important task within game playing to this day $[57,58]$. The dominance of the Mini-Max technique is supported by the powerful alpha-beta pruning strategy ${ }^{2}$, which can drastically reduce the size of its search space, achieving a greater "lookahead" in a game $[25]$.

In more recent years, other traditional games, such as the ancient Chinese game Go, have been explored in earnest, expanding the emphasis of research in the field well beyond its roots in Chess [46]. The need for new techniques to tackle this expanded problem domain have led to the introduction of powerful approaches, such as the popular UCT algorithm [19, 26]. The broad success of the UCT algorithm demonstrates the value in addressing a more diverse domain of problems in game playing, as techniques such as UCT, developed to achieve success in new areas, can advance the general state-of-the-art of the field.

While the direction of research has expanded from Chess to a wider variety of games, the majority of work in the field of game playing remains focused on twoplayer games [49, 63]. Multi-player games (where the term "multi-player" is here defined to refer to games with more than two players), such as Chinese Checkers, Focus, and multi-player forms of Go, have seen comparatively little examination. It is also noteworthy that techniques for multi-player games have been largely limited to

\footnotetext{
${ }^{1}$ Throughout this thesis, to simplify matters, we shall refer to the players with the masculine pronoun.

${ }^{2}$ All of these concepts will be explained in the body of the thesis.
} 
extensions of two-player game playing algorithms such as the Paranoid and the Max$\mathrm{N}$ approaches $[28,52,62,63]$. More critically, however, while recent years have seen more work dedicated to addressing the problem of multi-player game playing, multiplayer strategies have issues performing on a level comparable with their two-player counterparts for a wide variety of reasons, including involving unrealistic assumptions and the absence of good tree pruning schemes [52, 62, 65, 66, 75].

Despite this lack of focus within the literature, games that feature a number of players larger than two are, in fact, very common. The majority of commercial video games (a multi-billion dollar industry) fall into this category, with many players attempting to outplay each other, or with players seeking to work together within a coalition. Furthermore, a rise in popularity of more complex board games, such as the enormously popular Settlers of Catan, has helped to emphasize multi-player strategy games in the public eye [67]. Even considering only "traditional" strategy games, one will observe that many well-known examples involve multiple players, such as Chinese Checkers, Hearts, Cribbage, or multi-player variants of Go.

Given the prevalence of multi-player games in the real world, and the issues discussed above in handling the multi-player case with existing game playing strategies, it is a worthwhile endeavor to seek to improve existing multi-player game playing techniques. It is this problem which motivates the work presented in this thesis. We will furthermore demonstrate that, while our original motivating premise is the specific improvement of multi-player strategies, the novel methods that we have proposed can be extended, in the general case, to two-player environments as well. We will investigate these avenues of research, as well, in the body of this work.

\subsection{Problem Approach}

When considering AI game playing engines and strategies, for both two-player and multi-player environments, there are two distinct methods to achieve stronger performance. The first of these concerns the evaluation function, or selection criterion, as 
a more refined board evaluation strategy will, in turn, lead to better play. However, while highly-refined heuristics have been developed over the years for a range of wellknown board games (such as Chess), improvements of this variety, naturally, only apply to a single game. They can, thus, be classified as domain-dependent strategies.

The second approach is to search a larger section of the game space, which can be achieved through either a more powerful hardware platform, or through better tree pruning schemes so as to use our available hardware resources more efficiently. In the case of the alpha-beta search, this is usually achieved through the concept of move ordering, where we attempt to order moves based on how promising they are, leading to improvements to tree pruning $[48,54]$.

The second of these methods is particularly interesting, because while domaindependent techniques (that could lead to better move ordering and tree pruning) can certainly be envisioned, this category also includes domain-independent methods that have the potential to be applicable in a wide variety of games. Indeed, the alpha-beta pruning strategy itself, is one of these domain-independent techniques. Given that we are interested in improving the state-of-the-art of multi-player game playing techniques in the general case, such domain-independent schemes constitute the central focus of this thesis.

In this regard, we observe that the concept of move ordering is based upon constructing a ranking of available moves, and searching the best one first. Furthermore, in the specific context of multi-player game playing, we also have the ability to rank the opponents themselves. It is therefore reasonable to conclude, that novel, and effective, ranking mechanisms can be applied to improve searching in game trees, in the general case.

To this end, we consider the formerly unrelated field of Adaptive Data Structures (ADSs). The field of ADSs is based upon an observation related to certain forms of data structures, when one must, in some way, traverse through the data structure to find the element queried for, usually from the head (if a list) or the root (if a tree). While the position of the elements in such a data structure is irrelevant if all of them 
are queried with a uniform frequency, it is well known that this is not the case in the vast majority of practical cases $[20,21]$. In such scenarios, when the assumption of uniformity cannot be justified, it is clearly advantageous to place frequently-accessed elements near the head of the list or the root of the tree, so that they are located faster, and the less frequently accessed ones near the tail of the list or the leaves of the tree.

This would be the obvious solution if the access probabilities were known. However these access probabilities (frequencies) are, without loss of generality, unknown. Further, they may even change over time [21]. The field of ADSs, therefore, concerns itself with learning these frequencies, without resorting to average-based estimation, and with altering the data structure's internal arrangement to suit the access patterns. An ADS, then, is a data structure with some sort of update mechanism that reorganizes elements, usually whenever a query is made. A simple example (which will be explored in far more detail later in this work) is the "Move-to-Front" update scheme, which specifies that an element that is accessed is then moved to the front of the list [2]. The field of ADSs contains a great number of these update mechanisms, each characterized by its own benefits and drawbacks [13, 20, 21].

It is well known that that the mechanisms governing the operation of an ADS ensure that it converges to a structure that represents a specific ranking of the objects that it contains. While this ranking is specifically concerned with the access frequencies, we will employ ADSs here as a system for ranking objects in a search based on their importance, and construct techniques where the most important elements are "accessed" more frequently. Due to this natural ranking capability, and their general performance, we choose to employ ADSs to address the problem of improving performance in multi-player games. The work in this thesis represents the first combination of ADSs with game playing, in general, presented in the literature. 


\subsubsection{Verification of the Approach}

Given the known difficulties of performing a useful, theoretical analysis of game playing algorithms [54, 63], we have, instead, elected to demonstrate the efficacy of our new techniques, derived from ADSs, via practical experimentation in real-world game trees. This is precisely what is done by the vast majority of researchers in the field, given that existing theoretical results tend to be relatively weak, and not typically representative of how game playing techniques will perform in a "real-world" situations $[54,63]$. A broad range of configurations also allows us to observe the performance of our novel techniques in highly variable, and potentially unexpected situations.

To accomplish this, we have implemented a variety of intelligent AI game players, for both multi-player and two-player games, and have had them play a large numbers of games against each other, retrieving metrics on the efficiency of the search in each case. The specific experimental model varied depending on the objectives of our current work, and detailed descriptions of our technique are provided in each relevant chapter.

\subsubsection{Complexity of the Verification}

Rather than test our players in a single multi-player or two-player game, we have opted to implement a range of games, which are detailed in Appendix A. While experiments within a single game model would enable us to demonstrate the power of a novel technique in that specific domain, by employing a range of testing domains, we provide much stronger evidence of the reliability of our new techniques, and demonstrate that they continue to perform well under different configurations. Furthermore, the specific qualities of individual games lead to different pieces of information about the performance of varied techniques, in general.

It is extremely pertinent to mention that the implementation of such a range of multi-player, as well as two-player, strategic board games represents a substantial 
expenditure of effort. Quite modestly, stated, the implementation of each game has been a project-sized undertaking in its own right. First of all, we emphasize that each board game has completely distinct board representations implying that the so-called "knowledge representation" has to be unique for each game. Further, each game has its own rules, objectives, and potential moves available to the players. All of these issues must be properly accounted for within the game engine's design. Even the layout of the board can be highly variable, as while a checkerboard, or a grid of some sort, is a common arrangement, it is far from universal. For example, the game of Chinese Checkers, which we have employed in this work, is played on a star shaped board arranged as a series of hexes. Furthermore, in order to prevent implementation issues skewing our results, it was necessary to develop all these game models within the context of a universal engine, so as to ensure that "identical search" algorithms may be employed.

Beyond the need to account for the specific rules of the games in question, while the search techniques we examine and propose in this work are universal, they must be able to employ a heuristic, unique to the game in question, that correctly evaluates the board layout at leaf nodes [48]. While the objective of our research is not to create a world-class player for any of the specific games we have employed as our test basis, it was necessary to employ reasonable evaluation methods to insure realistic play, requiring an even greater level of effort and examination. Our evaluation methods are also described in Appendix A.

Any reader who has implemented or worked on real-life games will certainly appreciate the monumental amount of work that has been undertaken to inspect all these games in a real-world setting, and to test them with various heuristics, ADSs, and configurations. The statistical validation of the tests also represents a non-trivial undertaking. 


\subsection{Contributions of the Thesis}

Our work presented in the body of the thesis provides a detailed analysis of applications of ADSs to the field of multi-player game playing, and then extends these techniques to the two-player domain. We examine a wide range of different experimental configurations, to provide a complete and informative analysis of our new techniques in a wide range of situations. Specifically, we examine, in detail, the following:

- Use of ADSs to rank opponents in a multi-player game;

- Ordering moves through move history in two player and multi-player games using ADSs;

- Performance of ADS-based techniques in a range of game models;

- Applicability of ADS-based techniques in multi-player games with varying numbers of opponents;

- Effectiveness of ADSs at both initial board positions, and a wide range of reasonable mid-game board states;

- ADS performance in game trees of different ply depths;

- Relative strengths and weaknesses of a broad range of known ADS update mechanisms in game playing;

- A comparison of ADS-based techniques and the existing state of the art for two-player and multi-player games.

In the course of this exploration, we have developed distinct, novel move ordering techniques, which we have named the Threat-ADS and History-ADS heuristics. The first of these, the Threat-ADS heuristic, employs an ADS in conjunction with the Best-Reply Search, a state of the art multi-player strategy, to rank opponents based 
on the threat that they pose to the perspective player. It then uses this knowledge to achieve improvements to the move ordering. Our findings conclude that the ThreatADS heuristic is able to produce statistically significant, reliable improvements to tree pruning in a wide range of cases.

The second, the History-ADS heuristic, generalizes ADS-based techniques to both two-player and multi-player games, by employing an ADS to rank moves based on their prior performance in the game tree, which we refer to as move history. This is analogous to the strategy employed by well-known move ordering techniques, including the acclaimed Killer Moves strategy and the History Heuristic. Similarly, this information is efficiently employed elsewhere in the game tree to provide improved move ordering. As with the Threat-ADS heuristics, our findings demonstrate that the History-ADS heuristic is able to consistently produce reliable, and in some cases very large, improvements to tree pruning in a range of cases.

The specific contributions presented in each chapter of the thesis are summarized in the following section. The publications that have resulted from these works are mentioned in each respective chapter (the specific publications related to this thesis are: [37], [38], [39], [40], [41], [42], [43], and [44]).

\subsection{Structure of the Thesis}

Chapter 2: This chapter provides a survey of the field of two-player and multi-player game playing, as well as that of the improving agent, ADSs. In the context of each, a discussion is given of the state-of-the-art techniques, and the benefits and possible drawbacks or weaknesses of these approaches. Emphasis is placed on move ordering techniques for two-player games, adaptations of the Mini-Max approach to multiplayer games, and techniques for applicable adaptive lists.

Chapter 3: In this chapter, we present the idea of ranking opponents, through the use of an ADS, in the context of multi-player game playing. We introduce the concept 
of relative opponent threat levels, and show how an ADS may be used to learn these relative opponent threats. We then describe how these pieces of information can be used by a state-of-the-art multi-player game playing algorithm, the Best-Reply Search, to improve move ordering and, therefore, lead to a reduction in the search space and the number of nodes expanded. This specifies the Threat-ADS heuristic. In this chapter we especially demonstrate,that the Threat-ADS can produce a meaningful decrease in the tree size by incurring, essentially, no additional cost, in each of our examined multi-player games, with a range of opponents. We also show that it can produce improvements in tree pruning at both initial and midgame board positions.

Chapter 4: This chapter elaborates on the work presented in the previous chapter, exploring how the Threat-ADS heuristic performs in a wider range of environments, and with a spectrum of update mechanisms. We show that the Threat-ADS heuristic can reliably produce useful reductions in the tree size, and that these reductions also remain in deeper trees.

Chapter 5: Based on the successes of the Threat-ADS heuristic, and the established capabilities of the acclaimed History Heuristic, this chapter explores a method by which ADSs can be used to order moves based on move history. The reader will observe that this is the same metric that the History Heuristic and a number of other highly-regarded move ordering techniques employ. We develop a novel technique based on this approach, called the History-ADS heuristic, and demonstrate how it can achieve vast improvements in tree pruning in both two-player and multi-player games.

Chapter 6: This chapter continues our research into the History-ADS approach, exploring two possible refinements to the technique, so as to mitigate costs, and to improve performance. Specifically, we examine the effects of limiting the size of the ADS to improve the efficiency of the update mechanism, and in prioritizing information based on the level of the game tree at which it was gained.

Chapter 7: Based on the successes and strong performance of the History-ADS heuristic in earlier chapters, we compare it here to two well-known and acclaimed 
move ordering strategies widely employed in game engines. These strategies, which inspired the History-ADS' use of move history, are the Killer Moves technique and the History Heuristic. We demonstrate that not only is the History-ADS able to perform on their level, but that it can additionally outperform these techniques in a wide range of situations.

Chapter 8: This chapter concludes the thesis, summarizes our contributions, and proposes areas for future work. 


\section{Chapter 2}

\section{Survey of the Field}

\subsection{Chapter Overview}

In this chapter, we will present a survey of the current understanding and conventions of game playing, considering both two-player and multi-player scenarios. We will also report briefly on the relevant state-of-the-art AI techniques for solving or playing them. We will cover, as well, the field of ADSs, which serve as our improving agent. We begin our discussion with a very brief overview of the various classes of games, and game playing, in general, before discussing strategies for two-player games. We then move on to multi-player strategies, and include a brief presentation of stochastic methods for both classes of games, which are relevant to the field in general. We conclude with our survey of the available strategies from the field of ADSs, which we will employ later in this work. 


\subsection{Families of Games}

Before we can present our survey of strategies for two-player and multi-player strategic games, it is necessary to present some level of classification for different families of games, to better portray the varieties of scenarios to which our work applies. The field of Game Theory offers a rich classification scheme that meets our needs, and has a history of acceptance among the scientific and mathematical communities from which useful and interesting formal results can be drawn [18]. We present here a subset of the parameters of Game Theory that are necessary to classify those games that are important to the field of AI and our research.

Cooperative vs Non-cooperative Games: The first major distinction that we make between classes of games, is between those games that are considered cooperative, and those that are non-cooperative, or competitive. A cooperative game is distinguished from its non-cooperative counterparts by the fact that the players are encouraged to form mutually beneficial coalitions, in order to maximize their payoffs within the game. In such games, a single player does not necessarily emerge as a clear winner [18]. Cooperative games, including the well-known Stag Hunt game and the Battle of the Sexes, are studied not only in the context of game theory, but within the social sciences too, as useful models of mutually-beneficial interaction [60]. A well-studied example is the Commons Game, which has served as a learning and modeling tool for real-world resource management situations [23]. In this work, we are concerned with competitive, non-cooperative games, and thus, while cooperative games are an interesting field, we do not dwell on them any further. However, the use of the techniques introduced in this thesis (i.e., those "borrowed" from ADSs) can quite easily be applied here ${ }^{1}$.

\footnotetext{
${ }^{1}$ The area of applying the features of ADSs to cooperative games is an avenue we intend to pursue further after this doctoral research.
} 
Zero-Sum vs Non-Zero-Sum Games: Another important distinction in classifying two-player and N-player games is the concept of zero-sum games. In a zero-sum game, the gain of one player, by necessity, penalizes another player, or at least removes their potential future rewards [18]. By necessity, a zero-sum game is also a non-cooperative game between the players as a whole ${ }^{2}$. Most classical board games, as well as card games such as Poker, are zero-sum. From this basis, even stronger parameters can be derived. A constant-sum game is a zero-sum game in which all possible "points" are, at any time, distributed amongst the players, and the total number never changes. Another related property is whether a game is monotonic, in which case, once a player receives a number of points, they can never lose those points due to future actions. These qualities are important in multi-player game playing, and will be revisited later in this chapter [63]. Although all the games analyzed in this thesis will be zero-sum in nature, the application of the novel concepts introduced here to non-zero sum games, holds potential.

Perfect vs Imperfect Information: A game is said to have perfect information if the entire game state is known to all players at all times. Classical board games such as Chess, Backgammon, and Checkers are examples of perfect information games, as all the pieces are in the open and can be observed freely by both players. The most common class of games that do not have perfect information are card games, in which one player's cards are kept secret from the opponents [18]. A related, although distinct, concept is that of complete information vs incomplete information. These refer not to the game state, but to the available payoffs, where in a game of complete information, the payoffs for all actions are known and certain, while this is not the case when dealing with scenarios of incomplete information. An example of where one encounters incomplete information is seen when a player has to draw a card from a deck. Clearly, the identity of the card to be drawn, and thus the payoffs of the move, are unknown. While we will discuss methods to handle games of these varieties later in this chapter, the work in this thesis is concerned with games of perfect, complete information.

\footnotetext{
${ }^{2}$ It is conceivable that in a zero-sum game, coalitions of players could act as a single unit, as is the case that occurs in Bridge. However, only one coalition, or team, can emerge as the final victor.
} 
Sequential vs Simultaneous Games: A less well-known but still very important distinction in Game Theory is between simultaneous and sequential games. Sequential games are those in which players take set turns in acting, and where there is some limit on the number of actions a player can take in any given turn [18]. Thus, all the classical board games mentioned thus far, fall into the class of sequential games. Simultaneous games are less common, and a well-known example of this class would be the Pursuers-Evaders game [6]. This game involves a group of pursuers chasing a group of evaders through a graph, with the evaders being able to move an unlimited distance through the graph, constrained only by the position of the pursuers. Thus, the objective of the pursuers is to systematically move through the graph, clearing all of its areas without allowing the evaders any chance to escape. As we are concerned with augmenting traditional game playing strategies, in this work, we concern ourselves exclusively with sequential games. Again, we believe that the potential of applying the novel contributions of this thesis to simultaneous games, is great.

Combinatorial Games: Combinatorial games are those games that have an expanding number of possible moves for at least one player, the nature of which depend upon those moves made previously in the game (normally thought of as changes in the board state) [18]. These are contrasted with simple games, such as the Prisoner's Dilemma, where even if the game repeats, each player only has two possible moves and where each of these two moves returns a fixed reward. There is a further distinction to be made between combinatorial games and combinatorially explosive games, where the number of possible moves and board states are so great that it becomes infeasible to calculate all possible board positions. Popular board games such as Chess, Checkers, and Go are combinatorially explosive, and are the primary focus of research in AI game playing, and in our work in this thesis.

As the reader can infer from the above, the specific family that our current research deals with, are non-cooperative, zero-sum games, which further involve perfect, complete information, and that are sequential, and combinatorially-explosive games. This category contains the vast majority of well-known, classical board games, which do not include dice or cards. Examples include the well-known Checkers, Chess, 
and Go, as well as modern, commercially-successful board games, such as Settlers of Catan [67].

\subsection{Game Playing: Overview}

Before we detail specific strategies, in this section, we will briefly discuss some concepts related to AI game playing as a whole. Thus we visit its historical context, and the phenomenon of game trees, and the need for branch and bound strategies, in game engines.

\subsubsection{Historical Overview}

The idea of designing machines and algorithms intelligent enough to play strategic games competitively against human players, has a substantial history in culture and the computing sciences. This history far predates the advent of practical game-playing engines. Indeed, in the early 19th Century, a game playing automaton named "The Turk" was presented as being capable of competitively playing Chess, and played against many famous monarchs and dignitaries of the time [56]. While, obvious to us now, this machine was a hoax, it demonstrates the interest that intelligent game playing holds, even to those who are not involved in the field. It is no coincidence that many of AI's most recognizable and popular achievements, such as Deep Blue defeating the world Chess champion Gary Kasparov in 1997, and IBM's Watson defeating Ken Jennings on the game show Jeopardy in 2011, are in the field of game playing [59].

Attempts to create a true Chess playing machine date back as far as 1914, with automata that could play a Chess endgame, at the point where explicit rules could be defined for the best possible move. However the major premises of intelligent game play were introduced by Shannon in $1950[48,58]$. The methods proposed by Shannon still, indeed, provide the basis for many state-of-the-art combinatorial game 
playing techniques, which will be detailed presently. While this work established the theoretical basis of a Chess engine, it was not until the 1970s that Chess playing computer programs came into existence, which were accessible to everyday consumers. Further, it was not until Deep Blue's landmark victory over Kasparov in 1997 that one could emphatically confirm that a computer could play Chess at the level of a world champion [59]. The victory of Deep Blue preceded a branching out of the field to areas outside Chess, including multi-player games, and necessitated new techniques. These set the context for the work we undertake here, as well as a vast amount of modern game playing literature.

\subsubsection{Game Trees}

Before we describe techniques for playing combinatorial games, we must briefly discuss the well-known concept of game trees, which are the standard by which the possible moves in an $N$-player sequential game are represented. A game tree is constructed by taking the starting, or current, state of the game as the root of the tree, and then considering all possible moves for the current player as branches from the root. This operation is repeated at each of the resultant nodes, until a terminal state is reached (usually, when a player has won, or a draw has been reached). These terminal states then become the leaves of the game tree.

To serve as a simple example of a game tree, we present a simplified version of the mathematical strategy game known as Nim, or the Subtraction Game. In this version of the Subtraction Game, two players alternate taking one or two sticks from a pile of five sticks. The player who is forced to take the final stick from the pile loses the game. The complete game tree of this five-stick variant of the Subtraction Game is shown in Figure 2.1. 


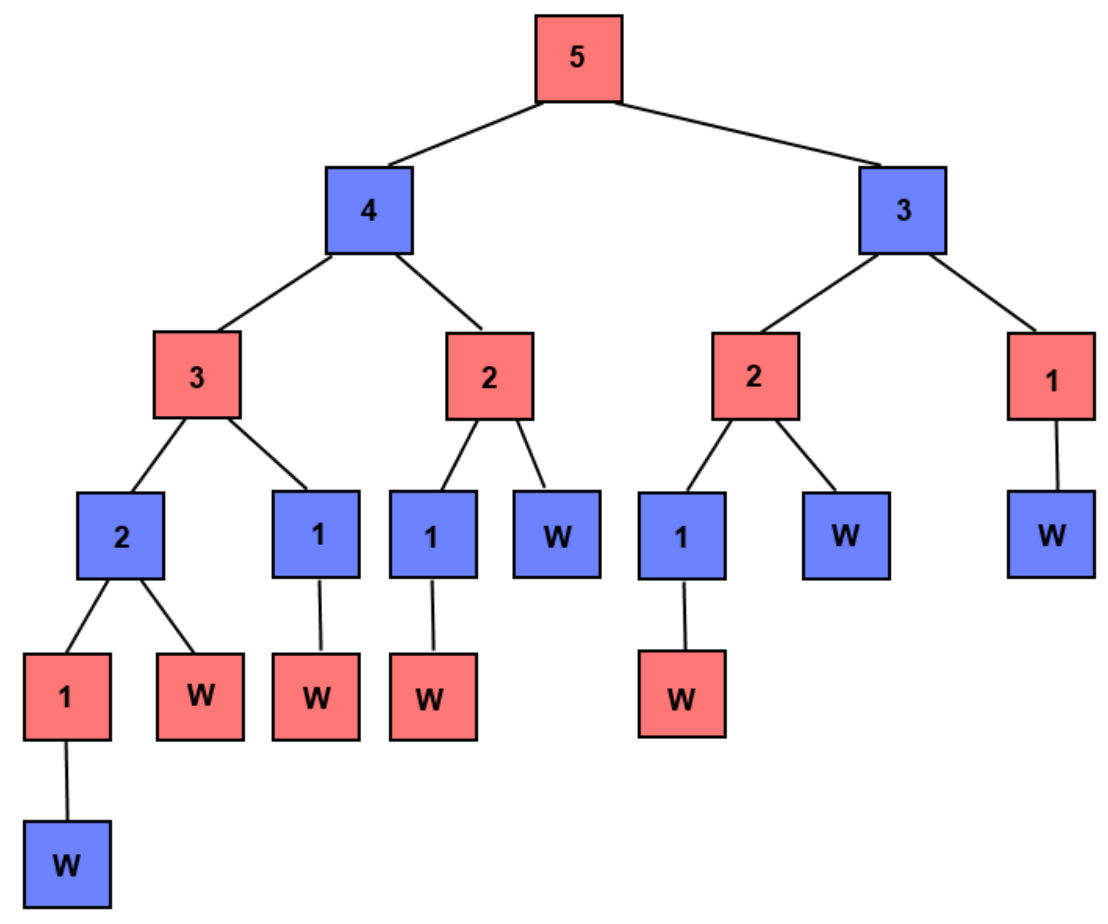

Figure 2.1: The complete game tree for the two-player five-stick Subtraction Game. Nodes where it is Player 1's turn are highlighted in red, and nodes where it is Player 2's turn are highlighted in blue. The leaves of the tree (where there are 0 sticks remaining) are coloured red when player 1 wins, and blue when player 2 wins.

\subsubsection{Branch and Bound}

In the case of a simple game like Nim, it is trivial to see how the first player can always force a win, as the entire game tree is available to any player. However, in the general case, it is not practical to construct a complete game tree for a combinatoriallyexplosive board game, as the size of the complete tree renders this operation infeasible [48]. Indeed, Shannon, in his original paper, estimated the size of the game tree of Chess to be approximately $10^{120}$ (the so-called "Shannon Number"), well outside the realm of feasibility [58]. Thus, it is necessary to bound the depth of the tree in some 
way, resulting in leaf nodes representing a midgame state, rather than a terminal position. To evaluate these leaf nodes, and how good they are for any given player, game engines employ evaluation functions, which return a value that is higher, for a given player, when the board state is estimated to be positive for them. An example of this, would be a summation of pieces, by relative value, on the board, in a game of Chess, as was proposed in Shannon's paper [58]

The use of these evaluation functions allow us to meaningfully search a feasible game tree. However, many branches of a game tree are unlikely to be very strong, and in fact, can be cut off through the use of bounds [48]. A combination of strong heuristic functions, and efficient bounding strategies, make up the core of traditional AI game playing algorithms.

\subsection{Two-Player Game Playing}

Now that we have introduced the concepts of game trees, and the ideas of heuristic evaluation functions, and branch and bound searches techniques within these trees, we will now present a survey of historical and modern strategies for two-player game playing. Specifically, we focus on deterministic strategies in this section, and discuss stochastic methods, such as the recent UCT algorithm, later in this chapter.

\subsubsection{The Mini-Max Algorithm}

By combining the use of evaluation functions, with the idea of game trees, Shannon proposed a conceptual Chess playing program, based on some earlier work done in game theory by von Neumann and others, which is currently referred to as the MiniMax algorithm [58]. While the Mini-Max approach is a well-known strategy, which has seen many refinements in terms of efficiency and speed, it serves as the basis for many modern game playing techniques [48]. The Mini-Max algorithm considers a limited game tree, usually with a certain preset depth (commonly referred to as 
"ply"), where the leaf nodes are assigned values from a given evaluation function. The algorithm operates on the assumption that the opponent's evaluation function is as strong as that of the perspective player. It thus assumes that when the opponent has the opportunity to make a decision in the game tree during his turn, he will choose the path that will give the lowest possible score to the perspective player, thus minimizing the score. Conversely, it is known that the perspective player, when given a choice, will choose the move that grants him the highest possible score, or maximizing it.

Following this rule, the Mini-Max algorithm proceeds in the same manner as a Depth-First Search of the game tree would, limited by the ply depth. When all of the branches of a node are assigned a value, the minimum value is chosen for that branch as a whole, if it represented the opponent's turn, or conversely, the maximum, if it represents the perspective player's turn. This sequence is repeated until the root node has a value, at which point the perspective player can determine which path in the game tree is the most favourable. For further clarity, a sample complete Mini-Max tree is shown in Figure 2.2.

\subsubsection{Alpha-Beta Search}

It is intuitive to see that the greater the search depth is in a Mini-Max search, the better the perspective player will play. This is because he will have access to more of the information resident in the complete game tree, from which he will be able to make a strategic decision. In order to search more nodes in a smaller time, naturally, greater computational resources are required. However, it is also possible to employ a branch and bound strategy, to limit the cost of searching to a given depth, and the most well-known method for improving a Mini-Max search, is the alpha-beta search $[25,48,63]$.

Instead of maintaining a single value, which is passed up the tree, the alpha-beta search maintains two values, $\alpha$ and $\beta$, which together provide a bound on the possible 


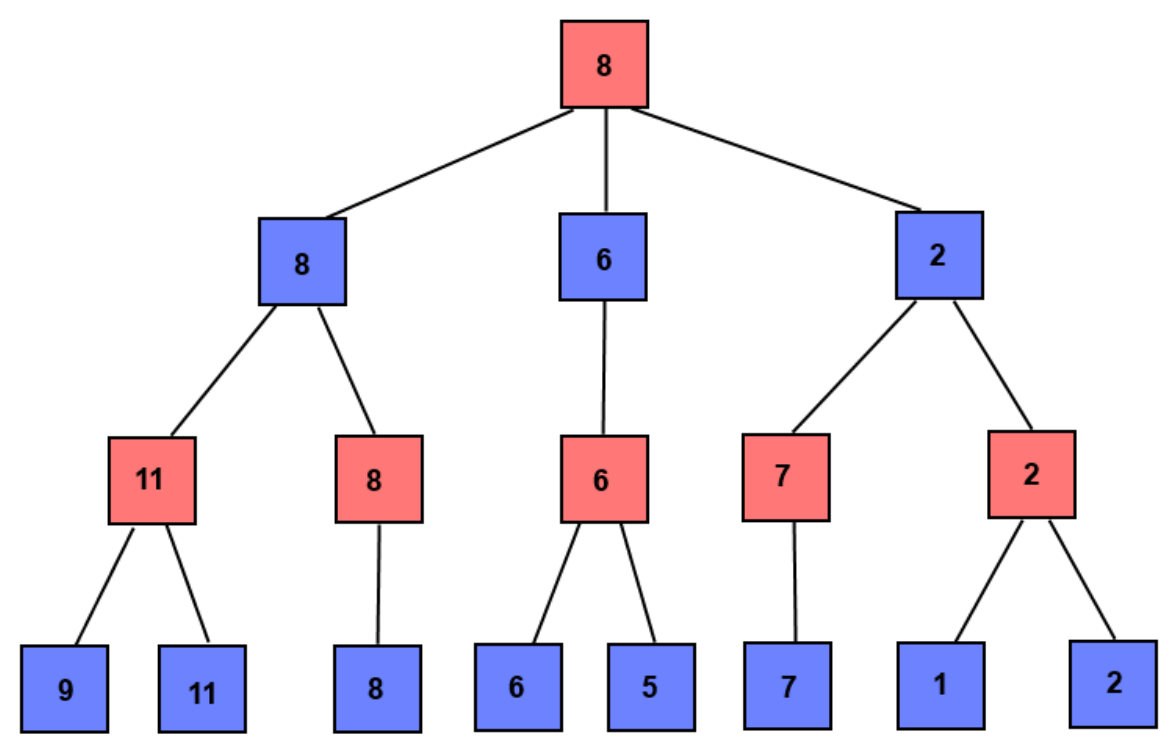

Figure 2.2: A sample Mini-Max tree. Nodes that are tinted red are Max nodes, and nodes that are tinted blue are Min nodes.

values of the Mini-Max tree at any given point. At any given juncture, $\alpha$ represents the minimum value that the player can expect to receive, and $\beta$ represents the maximum value the player can expect to receive. If it is ever the case that this bound is reversed, or has a range of $0(\beta \leq \alpha)$, then one can infer that better options exist for the player at other pre-explored nodes. Thus, the current node cannot provide the value of the tree as a whole, and consequently, it may be pruned immediately[4, 25]

In the worst case scenario, it is possible that the bounds will never be reduced to 0 or be inverted, implying that no time will be saved at all, and that the number of leaf positions evaluated will be $O\left(b^{d}\right)$, where $b$ is the branching factor and $d$ is the depth of the tree - which is exactly what is encountered in the naive Mini-Max scheme [4]. However, in the best case - achieved by always exploring the best (or worst, in the case of minimizing turns) move first - it has been shown that the number of leaf positions evaluated is $O\left(b^{d / 2}\right)$, which implies that with the same level of computation, 
the search could be extended to be twice as deep [25]. In the case of most game models, this can lead to a significantly more intelligent game playing algorithm. As we will refer to it several times throughout the thesis, the formal $\alpha-\beta$ procedure is shown in Algorithm 2.1.

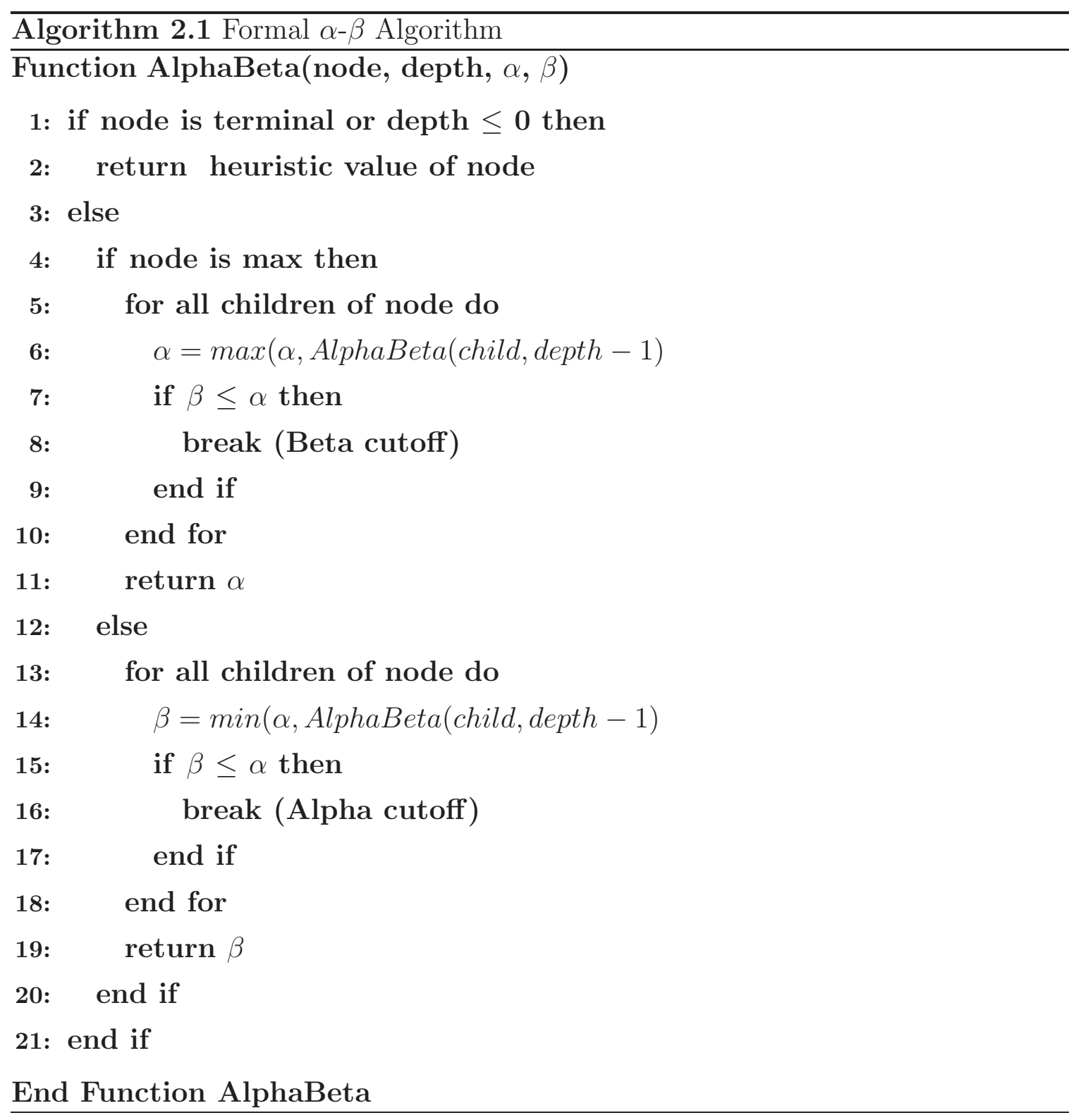




\subsubsection{Move Ordering}

With a little insight we see that the alpha-beta search benefits from always searching the best possible moves first, as this leads to a tighter bound, and to a more efficient pruning scheme [25]. However, if we were aware of the best possible move at each level of the tree, there would be no need to search at all. Consequently, estimating the correct move ordering is an entire category of potential improvements to the alphabeta search. The simplest way of achieving this is to use knowledge of the rules of the game, and to create rules that explore moves that are likely to be the best, first. For example, in Chess, moves which capture pieces could be explored first, as they are far more likely to be considered "best" by most reasonable Chess heuristic algorithms than those that do not. Of course, this tactic does not translate well to other games, and thus, effective move ordering strategies that do not rely on expert knowledge, are particularly valuable.

One method that can accomplish move ordering in the general sense, is related to the concept of iterative deepening. Iterative deepening is an extension to the DFS designed to give it some of the properties of a breadth-first search (BFS), including that of allowing it to function as an any-time algorithm [45]. Iterative deepening works by running the DFS algorithm repeatedly, and increasing the depth each time it is repeated. The intention of this exercise is that if processing time runs out, the program can return the result calculated from the previous level [45]. As alpha-beta pruning applied to Mini-Max is essentially a DFS-style algorithm, it is easy to see how iterative deepening can be applied to it, and indeed, it often is $[48,52]$. The previous results from the last iteration can be applied to achieve move ordering, as each node above the lowest level already has a score associated with it, and can be explored in the order of these scores, with the expectation that the final board state will trend near to those scores [45]. The optimal path from the previous level, often called the Principle Variation move in the literature [52], will thus be the leftmost branch of the tree. As this style of move ordering is completely domain-independent, it can be considered quite valuable. 
A wide range of domain-independent move ordering strategies have been introduced, such as the CLAMP system, which divides a game's board into dynamically ordered chunks, or the aforementioned Principle Variation move [12, 48, 52]. Two particularly well-known and studied strategies of this nature, are the Killer Moves technique, and the History Heuristic, which are closely related to our work in this thesis, and are thus afforded more detail presently. These techniques are related, in that both attempt to remember effective moves encountered ("effective" being defined as those likely to produce a cut, resulting in a smaller tree), and to explore them first if they are encountered elsewhere in the tree. Indeed, the History heuristic is often seen as a generalization of the Killer Moves heuristic, from a local to a global environment, within the tree. However, in many situations, they are also used in tandem $[52,54]$.

\subsubsection{Killer Moves and the History Heuristic}

The Killer Moves heuristic (also sometimes called the Killer heuristic) operates by prioritizing moves that were found to be good (that is, that produced a cut) in sibling nodes. For example, in the case of Chess, if it was found at some level of the game tree that White moving a bishop from $\mathrm{C} 1$ to A3 produced a cut, and that same move is encountered in another branch at the same level of the tree, it will be examined before other moves [54]. The heuristic is based on the assumption that each move does not change the board state that much. Therefore, if a move produced a cut in another position, it is likely to do well elsewhere, even if the preceding moves are different. Of course, this means that the Killer Moves heuristic will be less effective in games where single moves do, in fact, produce large changes within the game.

The Killer Moves heuristic accomplishes this prioritization by maintaining a table in memory that is indexed by the depth. Within each memory location, a small number of "killer" moves are maintained (usually two or at most, three) [54]. If a new move produces a cut at a level of the tree where the list is full, older moves are replaced according to some arbitrary replacement scheme. When new moves are 
encountered, the "killer" moves in the table at the current depth are analyzed first, if they are applicable. Note that, as the algorithm can check if the killer moves are available when expanding a new node, and examine them immediately, the Killer Moves heuristic does not require the nodes to first be sorted. In fact additional time can be saved by not even generating the remaining moves if one of the killer moves produces a cut. Therefore, the Killer Moves heuristic is particularly effective.

The History Heuristic is an attempt to apply the Killer Moves heuristic on a global scale, allowing moves from other levels in the tree to influence decisions. While a simplistic approach would be to maintain only a single list of "killer" moves and to apply it at all levels of the tree, this would allow moves that produce cuts near the leaves (as there will be many more of them, due to the explosive nature of the game tree), to have a disproportionate effect on the moves within the list. The history heuristic therefore employs a mechanism by which cuts produced higher in the game tree have a greater impact on deciding which move to analyze first.

This is accomplished by maintaining a large array, usually of three dimensions, where the first index is 0 , for the maximizing player, and 1, for the minimizing player. The next two indices indicate a move in some way. For example, in the case of Chess, the array is normally indexed by [from][to] where each of [from] and [to] are one of the 64 squares on the board. Within each of these cells is a counter, which is incremented when the corresponding move is found to produce a cut [54]. This counter is incremented by the value depth $*$ depth, or $2^{\text {depth }}$, thereby insuring the value increases more if the cut is higher in the tree [54]. When moves are generated, they are ordered by their value in this array, from the greatest to the least. In this way, moves that have produced a cut more often, and moves that produced cuts higher in the tree, are examined first.

The History heuristic is a particularly effective move ordering technique, especially when paired with Transposition Tables [54]. However, it does have some drawbacks. The array of counters that it stores is relatively large, although not a serious problem 
for modern computers, being two 64-by-64 arrays in the case of Chess. More importantly, unlike the Killer Moves heuristic, moves cannot be generated from the History heuristic; they must be sorted, thus adding non-linear time at every node in the tree. Consequently, it is desirable to look at other heuristics if they are capable of cutting branches of the tree without adding this additional sorting time. Furthermore, in very deep trees, the History heuristic is known to become less effective, to the point where modern Chess-playing programs either do not use it, or limit its application $[57]$.

\subsubsection{Other Alpha-Beta Related Techniques}

Aside from move ordering strategies, which this work is directly concerned with, and to which we pay special attention, a number of other possible improvements, or alternatives, to the alpha-beta search have been proposed over the years. While we do not deal with these directly in our research, we will detail a number of them here, for the sake of completeness. The methods that we propose could, however, be useful to enhance these in the future.

\subsubsection{Transposition Tables}

The use of transposition tables is a method to prune game trees. This is sometimes considered to be a form of move ordering, although it does not interact with the $\alpha$ or $\beta$ values. It is, however, capable of producing significant reductions in the size of the search space. Transposition tables are based on the observation that, in fact, the game tree is in its general form a graph, rather than a tree, as the same position may occur more than once within the tree. A transposition table is, therefore, a large, direct-access table, such as a hash table, wherein values for previously known board positions are recorded, and indexed by some encoding of the board position [7]. Thus, when a move that is already located within the transposition table is

encountered again, rather than explore it to depth 0 we may substitute the value 
from the tree, potentially saving a considerable amount of time, if this move was located close to the root [7].

While being conceptually very powerful, transposition tables suffer from some drawbacks, in their implementation. As can be expected, having a large table, with many possible address locations, results in better play. But due to the vast number of board positions in most interesting board games, collisions can and will occur at some point, regardless of how good the hashing function is [7]. Therefore, not only do transposition tables require a noticeable amount of memory, but they also require a method for collision resolution. Collisions cause two kinds of errors, the first being when a position being analyzed hashes to the same value as a position in the table, thus implying that it can be replaced by the value from the table, although the positions are not the same [7]. This can normally be resolved by examining the legality of the board position in question. The second error occurs when trying to store a new board position when its position in the transposition table is already occupied [7]. In this case, the question of which value is to be kept must be determined through a so-called replacement scheme.

Many replacement schemes exist for transposition tables. Exhaustively detailing them individually is outside the scope of this work. However, a particularly popular method is the "Deep" and associated "2-Deep" schemes, which are relatively easy to implement and effective. The "Deep" method maintains additional information about the depth in the tree where the board position was encountered, and keeps the one encountered closer to the root, with the hope that it would generate more savings if the board state is more common higher in the tree [7]. "2-Deep" is related to the "Deep" scheme, except that this time a two-level transposition table is employed, and potentially two board states are held at each level. The "2-Deep" rule is commonly applied in modern game engines and has been found to be particularly effective $[52$, $54]$. 


\subsubsection{The Horizon Effect and Quiescence Search}

The so-called "Horizon Effect" is an identified problem with Mini-Max style algorithms, where a sequence of moves leads to an inevitably bad position. The issue arises, when it is possible to delay the outcome of that bad position, for example, by repeatedly checking the king in Chess, sometimes long enough that the ply depth is used up [48]. A potential solution to this problem is referred to as "quiescence search", where moves that are "noisy" (they themselves appear good, but all moves around them look to be bad) are investigated further with a localized leaf search, in an attempt to identify delaying tactics and modifying the seemingly-good value of the node [51]. Quiescence search is a very natural extension of Mini-Max, as it is achieved by simply running the search again at a leaf node until that leaf node becomes "quiet". As with iterative deepening, the fact that the search is combinatorially explosive means that, for a reasonable number of "noisy" positions explored, to a reasonably small ply depth, the running time of the algorithm will not increase by more than a constant factor.

There are some difficulties that can arise with the implementation of quiescence search in certain domains. Some games can possess the property that potential delaying tactics can go on for quite a long period of time, and thus a move remains "noisy" for a period longer than expected, resulting in delays [51]. For games that are generally considered to be "difficult", a good solution to this remains an open problem. However, a fixed depth allowable for the leaf searches can, at least, prevent the algorithm from running for too long, as described in the previous paragraph.

\subsubsection{Stochastic Improvements}

A significant advantage of the alpha-beta search, and why we have referred to it earlier as a "deterministic" method, is that, for a given ply depth, it will always return the best possible move under our evaluation function. It thus does not make "mistakes" [25]. Phenomena such as tree pruning, move ordering, and transposition 
tables improve the performance of the deterministic algorithm, without altering the value of the tree as a whole, or, implicitly, the AI's decisions. However, we may arrive at a point where it becomes unreasonably difficult to achieve further improvements, or to a game state at which some "common-sense" decisions can be made about what is a bad move without considering them further. It is then possible to immediately prune moves that are considered bad due to expert knowledge, or by invoking statistical foreknowledge of the game, although this does eliminate the algorithm's deterministic guarantees [48]. For example, in the multi-player game Chinese Checkers, it is almost never advantageous to move backwards, although the rules allow it. Thus, a strict heuristic could immediately prune all nodes representing backwards moves.

A general-purpose implementation of this sort of forward pruning can be found in the PROBCUT algorithm, which achieves forward pruning not by a strict, gamespecific heuristic, but by statistical inference [8]. The PROBCUT algorithm works by performing an initial search at a lesser ply-depth in its completion, then running the main, deeper search. When a node that was a leaf in the original search is considered, its heuristic value in the shallow search is used to calculate the probability that it lies outside the current $\alpha-\beta$ window of the algorithm [8]. If this derived probability falls outside a certain threshold, the node is immediately pruned, thus permitting the algorithm a deeper search in other regions of the game tree. Given the simplicity of its implementation, the PROBCUT algorithm, has been applied in conjunction with other methods described here to achieve even better strategies in recent game-playing algorithms [53].

\subsubsection{Handling Imperfect Information}

Given that the Mini-Max paradigm operates on building a game tree to achieve a level of look-ahead in the game's state space, it is directly concerned only with games of complete, perfect information. However, it is possible to fill in the blanks created by imperfect or incomplete information, assuming that the game is (like most games of chance) limited in what the unknown information could possibly be. An extremely 
simple way to do this would be to simply randomly fill in the missing nodes. For example, in a card game, one can quite simply, randomly determine the opponent's hand, although this has obvious problems and as such, it is not seriously used outside of considering the theoretical applications of deterministic algorithms to these games [63].

A more reasonable attempt to overcome this weakness is found in the Expectiminimax algorithm. The Expectiminimax algorithm creates "chance nodes" at each point in the tree where unknown information is present, and then continues based on the expected value of these chance nodes [48]. It can also be beneficial for certain games which utilize Expectiminimax trees to maintain a limited set of chance nodes, rather than a single value, while traversing deeper into the tree, so as to capture potentially catastrophic situations. Such a strategy, of course, increases the computational requirements of the game engine [51].

Another possibility in handling imperfect-information games is to perform MonteCarlo sampling, a technique detailed in Section 2.6. There are also other techniques that can be applied to games with imperfect or incomplete information, such as neural networks and fuzzy decision trees, that can outperform Mini-Max variations, but we will not go into any further depth with these methods due to their lack of relevance to the work presented here [48].

\subsubsection{Non-Alpha-Beta Strategies for Two-Player Games}

While the alpha-beta search has a very strong historical basis, and remains in use in a variety of forms in cutting-edge research today $[48,52]$, there are other techniques to enhance Mini-Max style search that are in use, and that can perform better for certain games or under certain circumstances. We will briefly discuss some of these here. 
One such alternative is the Scout algorithm, also referred to as Principal Variation Search (Distinct from the PV-move in move ordering) [9]. The Scout algorithm functions by searching the leftmost node of any node immediately, and then attempting to obtain immediate pruning by considering the bounds constructed from that leftmost node [9]. This philosophy can perform better than alpha-beta pruning if and only if the moves are properly ordered, which can be enhanced through techniques such as iterative deepening, as it assumes that the leftmost node creates a good bound, and it needs to re-scout if another node disproves this hypothesis. Given that it is highly dependent on move-ordering, it functions much better in the case of games for which strong move-ordering techniques are known, such as Chess [48]. The methods for inferring move ordering proposed later in this work could also be potentially applicable here.

Another potential improvement on alpha-beta pruning is the $\operatorname{MTD}(\mathrm{f})$ algorithm [24]. The MTD(f) algorithm is a breadth-first algorithm, that constructs a Mini-Max tree faster than alpha-beta pruning in cases where an existing transposition table or other expert information is available to direct the search, resulting in fewer leaf nodes being explored [24]. While it produces some improvements in Chess and other highknowledge games, its shortcomings should be clear from its description, as it is heavily dependent on expert knowledge and the breadth-first nature of the algorithm causes it to be significantly more memory intensive. Furthermore, there are considerable obstacles to extending the algorithm in terms of parallelization (even over alpha-beta search) and applying other useful techniques found in the field. Thus, as such, Scout variants remain state-of-the-art for Chess and most other high-knowledge games [24].

Both MTD(f) and Scout rely on an older alpha-beta alternative known as SSS* [36]. SSS* is similar to Scout and $\operatorname{MTD}(\mathrm{f})$ in that it assumes a good move ordering and directs its search along a beam of likely candidates [36]. In spite of the fact that the alternatives to beam search normally lead to stochastic improvements (similar to PROBCUT), it can be shown that SSS* dominates alpha-beta. In other words, it can be shown that SSS* returns a value equivalent to the alpha-beta search and that it will never search a node that alpha-beta will not [24]. However, SSS* has 
been neglected in favour of alpha-beta because of alpha-beta's generality, and the complexity of SSS*. As such, it is impractical to include a complete description of the SSS* algorithm and its derivatives, $\operatorname{MTD}(\mathrm{f})$ and Scout, and so the reader is advised to the technical report due to Plaat [36] titled "A Minimax Algorithm Better than Alpha-Beta? No and Yes" for a complete explanation.

\subsection{Multi-Player Game Playing}

In this section, we will detail the methods by which two-player game playing strategies can be extended, or adapted, to the $N$-player case. We will discuss briefly the specific challenges associated with multi-player games, and report the state-of-the-art strategies to play multi-player board games.

\subsubsection{Challenges of Multi-Player Games}

The principles of multi-player games do not vary substantially from those already laid out for two-player games. Indeed, multi-player games can still be easily categorized as zero-sum, perfect information, etc. The only difference is that the number of players is increased from two, to an arbitrary $N$ self-interested agents. However, the ramifications of this, in terms of strategy, are extensive, as we no longer have a solitary perspective player, and a single minimizing opponent.

There are two principles of game playing, in particular, that vary significantly when more than two players are involved, and both of these must be addressed. Firstly, in the domain of two-player games, non-cooperative games are quite simple, as every action that one player takes, by necessity, harms the other player [18]. However, in a multi-player game, even one with a single winner at the end and with no difference in reward for finishing in any other position, certain strategies may benefit more individuals than merely the player who takes the action [63]. Thus, temporary coalitions of players may arise. This can, in fact, be a valid strategy, even though 
such coalitions will inevitably fall apart $[63,74]$.

The other related factor that bears mention concerns the zero-sum nature of games. Similar to the previous point, while a multi-player game may be zero-sum when considered in its entirety, it is possible for individual actions to benefit more players than merely the player taking the move in question, or damage one of the players significantly more than another. This property means that it is not always sufficient to assume that each player is playing with the same, personally-optimal strategy, because a more complex "opponent modeling" may be necessary to avoid bad plays [65].

Despite these concerns, the strength of the alpha-beta search, and the vast body of research done on it, makes it desirable to extend it to the multi-player case. There are, in fact, several different ways by which the Mini-Max approach can be extended to multi-player games, each with its own benefits and drawbacks [62]. Introducing and comparing these extensions will comprise the remainder of this section.

\subsubsection{The Paranoid Algorithm}

The first, simplest attempt to extend the Mini-Max algorithm to multi-player game playing, results in what is commonly termed the "Paranoid algorithm" in the literature $[52,62,63]$. This approach is the most intuitive, and requires the fewest changes from the core Mini-Max algorithm. In the Paranoid algorithm, the score at leaf nodes remains the output of a specific evaluation function, as in the alpha-beta search. However, as the game is now of a multi-player nature, there will be multiple opponent turns in between each of the perspective player's turns. The Paranoid algorithm handles this by treating every opponent turn as a min node [62]. Thus, for a three player game, the Paranoid algorithm could be referred to as Max-Min-Min, or for a four player game, Max-Min-Min-Min. A sample game tree for the Paranoid Algorithm is presented in Figure 2.3. 


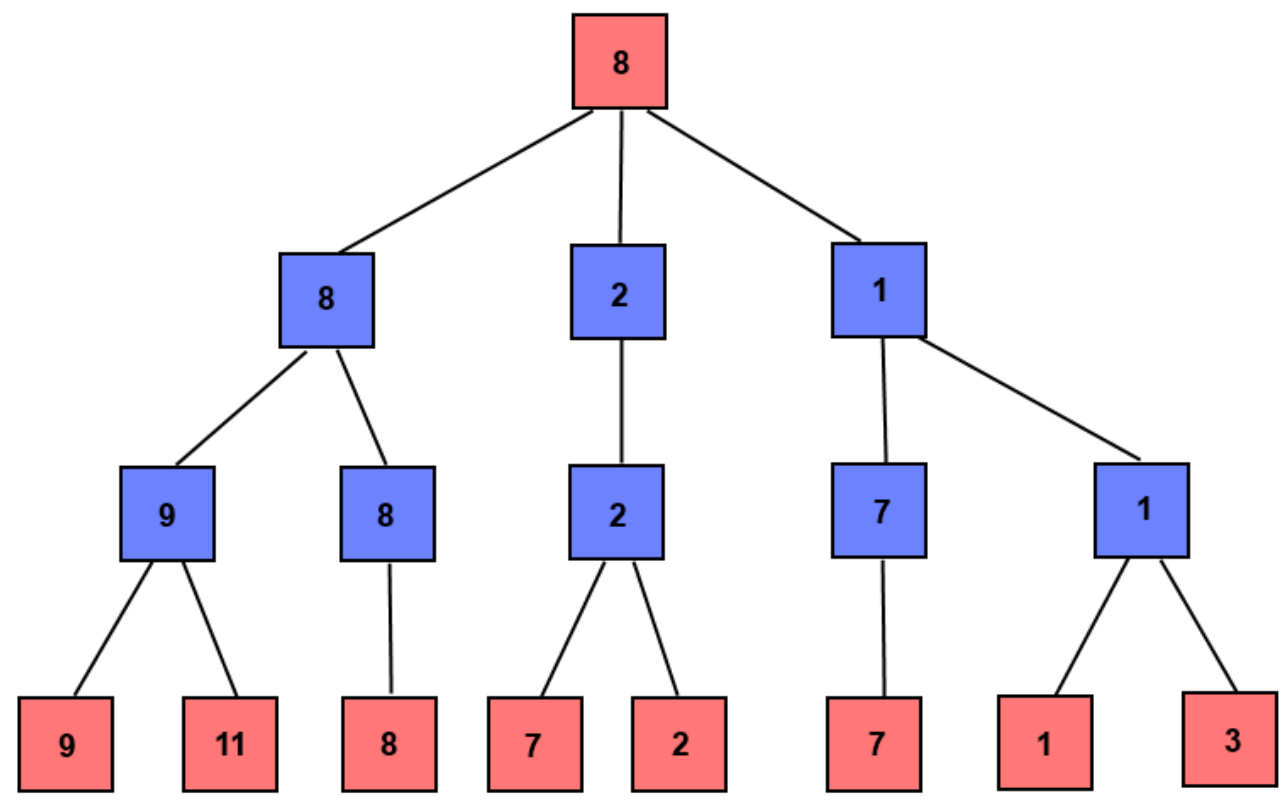

Figure 2.3: Sample Paranoid Tree in which the red nodes are MAX nodes, and the blue nodes are MIN nodes.

The Paranoid algorithm, therefore, gets its name from how it treats all opponents as an implicit coalition against the perspective player, and this leads us to its primary drawback. While it makes the "safest" decisions by assuming that all players will work against the perspective player, if the game has a single winner, and the objective is the same for all players, this assumption is, clearly, not reasonable [62]. Indeed, opponents will be assumed to do whatever harms the perspective player most, even if these moves harm themselves more! Furthermore, as there are multiple MIN levels in a row, the Paranoid algorithm affords worse look-ahead, at the same ply-depth, for the perspective player, and recent results show that the task of planning ahead for the perspective player is significantly more valuable to good play than the same level of planning ahead for the opponents [52]. Thus, it can lead to unrealistic play, or poor use of computational resources, relative to an equivalent alpha-beta search. 
Despite its obvious disadvantages, the Paranoid algorithm possesses some positive qualities as well, relative to other multi-player algorithms. Firstly, it is easy to understand, and extend existing alpha-beta implementations to handle multi-player situations. More importantly, its primary advantage is that a single value is assigned to each node, and thus, alpha-beta pruning may be used in conjunction with it. This is not the case for its most direct competitor, the Max-N algorithm described presently [63]. Although, its use of alpha-beta pruning is not as strong as in the two-player case, given that pruning can only take place on the boundary between Max and Min nodes, and never between multiple Min nodes, which represent the successive turns of the opponents [63].

\subsubsection{The Max-N Algorithm}

While the Paranoid algorithm is arguably the most intuitive extension of the MiniMax approach to multi-player games, another technique, called the Max-N algorithm, serves to more naturally model the idea of maximizing one's own score, in a multiplayer environment [28]. In the case of a two-player, zero-sum game, any gain made by one player must represent an equal loss for the other. Thus, an evaluation function that returns a single number can be employed. However, this is not the case if we wish to model each player to be maximizing his own score, in a multi-player environment. The Max-N algorithm resolves this by employing an evaluation function that returns a tuple of values of size $N$, where $N$ is the number of players, and where each value represents the evaluation of the board for a given player [28]. True to its name, the Max- $\mathrm{N}$ algorithm operates on the assumption that each player will choose the move that represents his greatest potential payoff (typically, player $i$ is represented by the ith value in the tuple), and this move, and the entire tuple, are passed up the tree towards the root [28]. Figure 2.4 shows a sample Max-N tree after the expansion of all the leaf nodes. 


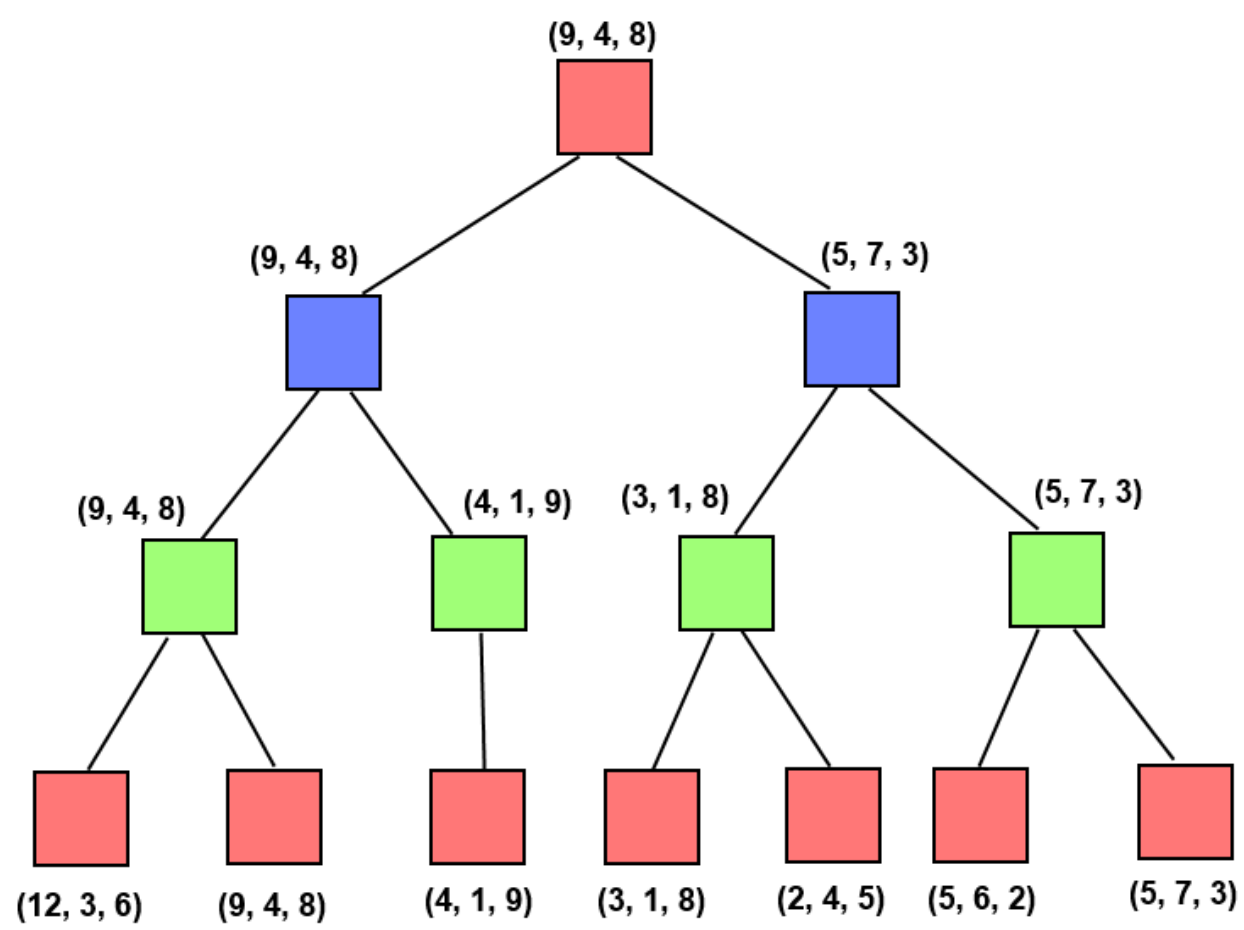

Figure 2.4: Sample Max-N Tree in which each colour represents a different player.

The Max- $\mathrm{N}$ algorithm can be said to be the formal generalization of the Mini-Max approach to an $N$-player game [28]. Indeed, a Max-N implementation will function identical to the corresponding Mini-Max search, should the algorithm be used on a two-player zero-sum game [63].

The fact that it represents "realistic" play, with each player favouring the move that most benefits his own score, is the most obvious advantage of the Max-N approach. This is because the more "relaxed" pressure on the perspective player allows him to take advantage of the various opportunities that may have been overlooked if the assumption of a "coalition of opponents" is made [62]. Unfortunately, aside from this, the Paranoid algorithm beats or matches the Max- $\mathrm{N}$ algorithm on nearly all other fronts. As with the Paranoid algorithm, Max-N suffers from poor look-ahead 
for the perspective player, and as it must run an evaluation heuristic for each player, rather than for a single opponent, it is necessarily computationally more expensive at the same ply depth.

The more important drawback to the Max-N algorithm, however, is its inability to attain alpha-beta pruning [63]. Pruning strategies do exist for the Max-N algorithm. However, these are based on constructing bounds on the range of values in a Max-N tuple. Furthermore, deep pruning is not possible under normal circumstances, as a node may be ruled out as the final value, but it may still influence its neighbors $[27,28]$. Those pruning strategies that do exist, perform better if the game in question has the properties of being a constant sum game, and/or monotonic in nature, because these characteristics influence the possible bounds on the Max-N tuple. However, given that this is an unlikely scenario, it is possible to prune speculatively, and revisit pruned nodes if it is determined to be possible that they could still influence the tree [63]. The end result is that while some pruning may be applied to the Max-N algorithm, it is far less effective than alpha-beta, and thus, the Paranoid algorithm can outperform it due to a deeper search, despite an unrealistic assumption [52, 63].

\subsubsection{Extensions to Max-N}

While not directly relevant to our work, there have been, more recently, two extensions to the Max-N algorithm proposed. These seek to remedy some possible weaknesses with the strategy. These are briefly summarized below.

An observed drawback to the Max-N approach is that it commonly breaks ties to the left. While this is no issue in the case of an alpha-beta search, as tied nodes are necessarily equivalent in a zero-sum game, this does not necessarily hold for Max$\mathrm{N}$. This is because nodes that have the same value for a given player may be very different for each of his opponents $[63,65]$. An extension to Max-N, called Softmax-N, attempts to address this [65]. Given two tied options, a player may have different priorities, in how they break these ties. He may simply break ties to the left, or at 
random, or he may favour the move that minimizes his strongest opponent as much as possible. The Softmax-N algorithm seeks to resolve this through opponent modeling, by attempting to determine, through observation over the course of the game, how each opponent breaks ties [65]. If a tie exists where we are unsure of the acting player's model, the algorithm maintains both of the nodes, passing a set of tuples up the tree, to insure the perspective player is aware of possible tiebreaking risks later on $[65]$.

Another approach, related to Softmax-N, is the Probmax-N strategy. Softmax-N insures that the player is aware of possible tiebreaking outcomes, but does not specify the likelihood of these events. Rather than maintaining a set of opponent models and using them only in the case of ties, Probmax-N maintains a set of opponent models and weights, so that for each action the probability of the action being taken by the player can be determined [66]. Thereafter, the value of each node is not selected from its children, but it is rather taken to be the expected value of that node [66]. This, arguably, provides the most realistic assessment of opponents' behaviour. But by necessity, the Probmax- $\mathrm{N}$ is unable to benefit from even the limited pruning available to the Max-N algorithm, as many nodes can contribute to the expected value.

\subsubsection{The Best-Reply Search}

The Paranoid and Max-N algorithms, and their variants, have remained the standard for deterministic multi-player game playing for many years. However, quite recently (February 2011), a new Mini-Max style multi-player game playing algorithm was introduced, which can, in some cases, significantly outperform both the Paranoid and Max-N algorithms. This is algorithm is called the Best-Reply Search (BRS) [52]. In the case of the BRS, all the perspective players' opponents are again considered to operate as in a coalition, as in the Paranoid algorithm. However, between each of the perspective player's turns, it allows only one opponent to act [52]. The opponent who is allowed to act is the one who has the most minimizing move to the perspective player at this point in time, which is why the algorithm is named the "Best-Reply Search". 
In essence, the scheme pretends that all opponents are not simply a coalition, but that the coalition represents a single player with significantly more resources available than what the perspective player possesses. Figure 2.5 shows a single level of a BRS tree (only a single level is shown for space considerations, as the branching factor is considerably higher for opponent turns in BRS), where the minimum of all opponent turns is being selected.

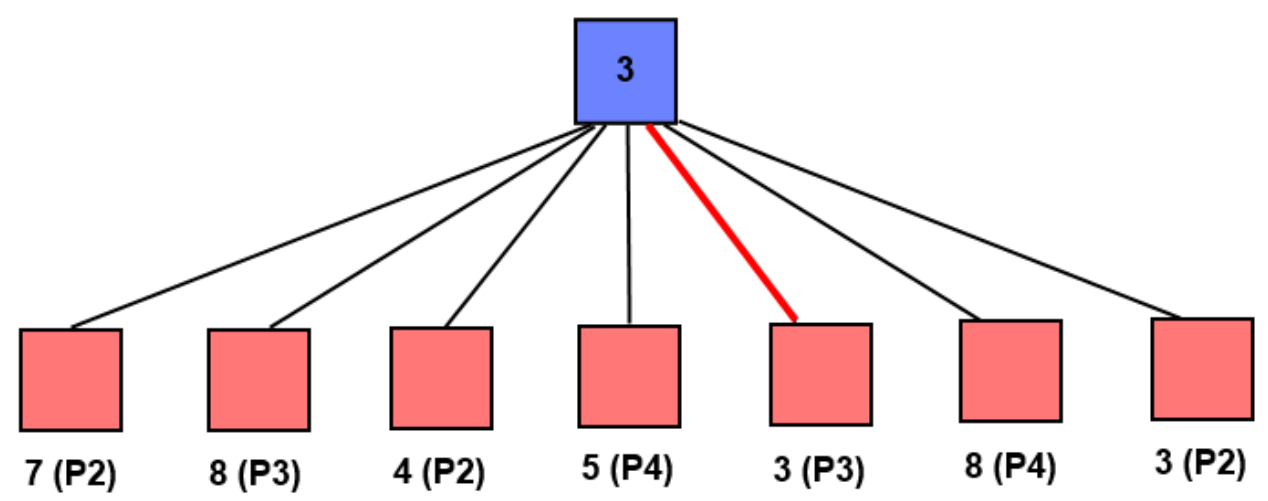

Figure 2.5: The operation of a single level of the Best-Reply Search. The scores that are reported have the opponent's player number listed next to them (in parenthesis) to assist in the clarification.

By necessity, the BRS considers illegal move states, and this is arguably its most serious limitation, as it, in fact, restricts the games to which BRS can be applied [52]. The BRS, in its originally proposed form, can only be applied to those games where it is meaningful for players to act out of turn, and performs best when the board state does not change too dramatically in between turns [52]. An example of a game to which BRS cannot be easily applied is Bridge, because scoring in Bridge is based on tricks, and thus, allowing players to act out of order renders the game tree to be void of meaning. In a game where the game state changes significantly between turns, there is a serious risk of the BRS arriving at a model of the game which is 
significantly different from reality. However, more recently, a proposed extension to the BRS, named $B R S^{+}$, was proposed, which seeks to remedy the issue of the BRS arriving at illegal game states. This is accomplished by allowing one opponent to make the best reply, as usual, but specifying that every other opponent is assumed to make a special move, which is determined based on a move ordering heuristic [16]. This move ordering heuristic can operate in a manner similar to either the Paranoid or Max-N algorithms, when choosing special moves [16].

However, in cases where it can be applied, the BRS has many benefits over the Paranoid and the Max-N algorithms. Indeed, it often outperforms them quite dramatically, as in the case of Chinese Checkers [52]. As can be intuitively observed, when all opponents are considered to be a single entity, the game is again modeled as a two-player game, and played using the Mini-Max algorithm. Thus, alpha-beta pruning, and all other two-player alpha-beta search improvements, can be applied without the need for multi-player specific alterations. Also, as each level alters between Min and Max nodes, the BRS has the best possible pruning of all multi-player Mini-Max style algorithms $[52,63]$. Secondly, as it simplifies things to work with a model analogous to a two-player game, the BRS also allows better look-ahead for the perspective player than either the Paranoid or the Max-N paradigms. The benefits of this look-ahead are considered to be a significant factor in the performance of the BRS over the Paranoid and the Max-N in a broad range of games [52].

The BRS will be central to the research that we have undertaken.

\subsection{Stochastic Methods}

In recent years, due to a need for strategies apart from traditional alpha-beta search in a number of domains, such as the Far Eastern board game Go, a new variety of game playing strategies has risen to prominence. This is caused by Go's exceptionally large branching factor, with a very large number of moves available to each player at each board position, limiting the potential search depth of the alpha-beta strategy. 
This is augmented by the fact that it is known to be difficult to construct a good evaluation function for its midgame states [71]. Thus, a new set of techniques, based upon random game playing, have been developed for this and other domains, which play games in a stochastic manner, as opposed to alpha-beta's deterministic approach. Our work in this thesis does not involve these stochastic methods, so they are only described here in brief.

\subsubsection{Monte Carlo Tree Search}

This family of strategies which have risen to prominence due to their success in handling the game of Go, are referred to as "Monte-Carlo Tree Search" (MCTS) techniques $[26,71]$. The basic idea behind MCTS is that one can avoid the need for a highly-refined evaluation function, by simply playing random games from leaf nodes in a tree, and then using the average number of wins and losses as a substitute for a heuristic [26]. Therefore, an extremely simple Monte-Carlo algorithm (although not strictly speaking MCTS) would be to simply play 50 random games from each end node in a Mini-Max tree, played to a certain ply depth, and to then treat the result as a heuristic value. Of course, while this avoids the need for a strong heuristic, it does compromise one of the advantages of deterministic, Mini-Max style methods. This advantage is that the result is always optimal for perfect-information games, according to its evaluation function, which represents a possible drawback of MCTS [63].

The benefit of a true MCTS algorithm is that rather than simply replacing the evaluation function with random game playing, the exploration of the tree is directed by it as well, focusing the search, as opposed to searching to a fixed depth [26]. In this way, each node is treated as a Bandit Problem, and MCTS algorithms balance between exploitation and exploration, according to the number of times a node is visited, and its so-called "Win Rate" [19, 26]. The most well-known example of an MCTS algorithm used in the literature, is the UCT algorithm, which has been applied to a wide range of games with a great deal of success $[22,68,72]$. 


\subsubsection{The UCT Algorithm}

The now-famous UCT algorithm, operates based on the UCTValue, which orders the nodes in the order in which they should be explored, and serves as a balance between exploitation and exploration [26]. At each iteration of the algorithm, we navigate from the root node, choosing the next node to explore based on its UCTValue, or, if it has not been explored, selecting it first [19]. Upon reaching the first unexplored node, the UCT algorithm plays one or more random games, from that position, and returns a Win Rate (WR). This WR is then passed up the tree to the root, and used to update the WR of its parents $[19,26]$. Fundamental to the UCT algorithm is the UCTValue equation presented below. As the reader will observe, it increases as the WR increases, but decreases the more times the node is visited. The exploration/exploitation balance is controlled by the constant $C$, where a larger value of $C$ indicates a greater exploration [64].

$$
U C T V \text { alue }(\text { parent }, \text { node })=\text { winrate }(\text { node })+C \cdot \sqrt{\frac{\ln (\text { parent.visits })}{n . v i s i t s}}
$$

The WR at a given node is stored in terms of the WR for the active player [26]. This is intuitive, as a player would be assumed to be more likely to choose a move that will allow him to win the game. The UCT Algorithm is normally given a pre-specified number of time steps for which it is allowed to run, during each of which a new node is expanded. This grants it the advantages of being an "any-time" algorithm [73].

The UCT algorithm has also been extended, with enhancements including various forms of quiescence search and forward pruning, as well as applications to a very wide range of games, rendering this to be a very active area of research [22, 46, 72$]$.

In terms of multi-player games, the UCT algorithm very easily extends to an $N$-player environment. Its extensions, however, do not have the same issues with pruning and poor play that extensions of the alpha-beta search do, which would necessitate the creation of a specialized multi-player UCT algorithm [64]. Due to this observation, recent attempts to apply the UCT algorithm to multi-player games 
have been made, with varying levels of success, after it was initially shown to be able to strongly outperform the Max-N algorithm in the context of Chinese Checkers [64]. It has since been applied to a number of multi-player games, including Military Chess, and the popular board game Settlers of Catan, with modest success, although necessitating specific refinements beyond the scope of our current discussion [67, 73].

\subsection{The Improving Agent: Adaptive Data Struc- tures}

In previous sections, we introduced the core concepts of both two-player and multiplayer games, as well as the established methods for playing them. We will now present the formerly unrelated field of Adaptive Data Structures (ADSs), and give an overview of its challenges and most common methodologies. The rest of this thesis describes methodologies by which game playing can be improved using the ADS-based techniques that are described in this section.

\subsubsection{Overview of ADSs}

It is well-known in the study of data structures that the access frequencies of the elements in the structure are not uniform [20, 21]. For example, in a linked list consisting of five elements, $A, B, C, D$, and $E$, in that order, the corresponding access probabilities may be $20 \%, 5 \%, 10 \%, 40 \%$ and $25 \%$. Using a traditional alphabeticallysorted singly-linked list, this would pose a problem, as the two elements accessed most frequently, i.e., $D$ and $E$, could be located at the rear of the list, thus requiring a longer access time. We can intuitively see and formally prove that another linked list, holding the same five elements in the order $D, E, A, C, B$ will achieve a faster average performance. Thus, by restructuring the list, one can obtain an improved functionality for the data structure. 
In the trivial example above, the reorganization is obvious, as the access probabilities are assumed to be known and stationary. However, in the real world, the access probabilities are usually not known when the structure is first created. The field of ADSs concerns itself with finding good resolutions to this problem [21]. As the access probabilities are not known, the data structure must learn them as queries proceed, and adapt to this changing information by altering its internal structure to better serve future queries [20]. Individual types of ADSs provide update mechanisms that lead to this sort of behaviour for the specific data structure.

The most trivial kind of ADS imaginable uses basic estimation (often called Frequency-Count) [2]. A Frequency-Count strategy simply maintains an access counter for each element, and reorganizes itself after each query, or at regular intervals [20]. The Law of Large Numbers confirms that this sort of ADS will, after a sufficiently large number of queries, converge to the optimal structure for a set of fixed access probabilities. However, this requires maintaining an additional access counter alongside each element in the structure, which may be prohibitively expensive [20]. Furthermore, even if this is deemed irrelevant given the power of modern computer technology, these data structures do not respond quickly to changes in the access probabilities for each element, as they are based on history.

Given this shortcoming of Frequency-Count style data structures, the majority of research into ADSs has focused on memoryless update mechanisms, or state-based ADSs which require significantly less memory than the Frequency-Count strategy $[2,20]$. As we will see, these update mechanisms tend to be very simple and easy to implement. However, their mathematical properties can render them to be quite interesting and powerful [2]. We will focus the remainder of this chapter on showcasing the most common of these mechanisms, with a particular focus on adaptive lists, given that they are employed in our research. 
It should be noted that the data structures presented here, by no means, represent an exhaustive exploration of all that is available in the field of ADSs. A very large number of update mechanisms could be proposed for even a simple singly-linked list, and many variants have been presented over the years. Therefore, our goal is to present a set of ADS mechanisms that represent a good "starting point" for merging the fields of game playing and ADSs.

\subsubsection{List-Based ADSs}

In this section, we will introduce ADS update mechanisms for list-based data structures, in both the singly- and doubly-linked cases. We will present examples, and important results, related to the well-known update mechanisms employed in our work.

\subsubsection{Move-to-Front}

The Move-to-Front update rule is one of the oldest and most well-studied update mechanisms in the field of ADSs $[2,20,47,61]$. Not coincidentally, it is also one of the most intuitive rules. As its name suggests, when an element is accessed by a query, in a singly-linked list, it is moved to the head, or front, of the list. Thus, if an element is accessed with a very high frequency, it will tend to stay near the front of the list, and therefore will be less expensive to access. Furthermore, in a list where each element has $O(1)$ pointers to the next element, the operation of moving an element to the front also requires $O(1)$ time, being a simple matter of updating a few pointers. An example of the Move-to-Front rule in execution is shown in Figure 2.6, where the reader can obtain clear picture of how it responds to queries and updates its structure. 
Given that elements are always moved to the head of the list, when using the Move-to-Front rule, the list changes quite dramatically in response to each query, which can cause worse performance in many cases [47]. However, it has been shown that the Move-to-Front rule provides a system that costs no more than twice that of the optimal ordering, keeping it competitive against other update mechanisms, which can outperform it, but do not offer this performance guarantee $[2,20]$. In the case of doubly-linked lists, the Move-to-Front is commonly called the Move-to-End, and operates by moving the accessed element to the end of the list from where the search is initiated, thus allowing for two different forms of prioritization [17].

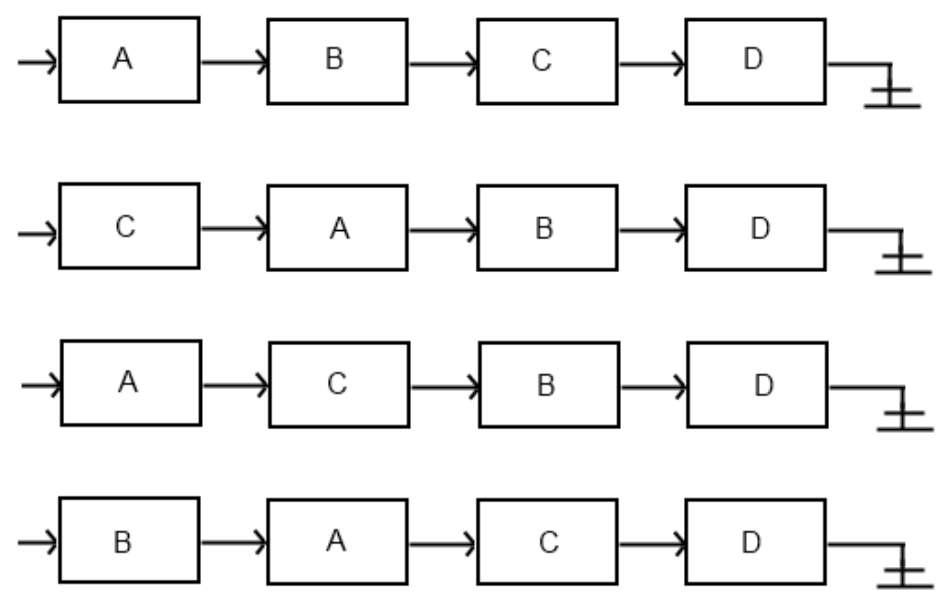

Figure 2.6: Execution of the Move-to-Front update mechanism for the singly-linked list $\mathrm{ADS}$ when the order of the elements accessed is $C, A, B$.

\subsubsection{Transposition}

The most common contrast against the Move-to-Front rule, also studied extensively in the ADS literature, is the Transposition rule $[2,17,20]$. When an element is accessed under the Transposition rule, it is swapped with the element immediately ahead of 
it in the list. Thus, as an element is accessed more and more frequently, it will approach the head of the list, which is in contrast to the in Move-to-Front, where it is immediately placed there. As can be deduced from its behaviour, the Transposition rule is less sensitive to change than the Move-to-Front rule, which, depending on the problem domain, can be a good or bad thing [2]. Under many circumstances, in fact, the Transposition rule will be much closer to the optimal rule than the Move-to-Front rule over a long period of time $[17,47]$. However, it can be shown that there exist situations where Move-to-Front is definitively better, and thus the question of which of these is to be employed depends on the problem domain [47, 61].

The biggest drawback that the Transposition rule has is a lack of any lower bound on cost, when compared to the optimal ordering [2]. Furthermore, arguments using amortized analysis have been made to show an improved performance from the Moveto-Front rule over a long period of time [61], although the definitiveness of these results have proven controversial $[2,17]$. Also, as one can intuitively imagine, the Transposition rule will take longer to converge to an optimal list arrangement, due to its incremental updates [17]. In the case of doubly-linked lists, the Transposition rule is commonly called the Swap rule, and operates by transposing the queried element one space towards the direction from where the search was initiated [17].

To showcase its execution, an example of the Transposition rule for a singly-linked list is presented in Figure 2.7.

\subsubsection{Move-Ahead-k}

The Move-Ahead- $k$ rule attempts to strike a compromise between the Move-to-Front and Transposition rules [21]. As its name suggests, when an element is accessed with a query, it is transposed $k$ spaces forward, or to the front of the list if $k$ spaces do not remain. Thus, the change is larger than for the Transposition rule, but not as dramatic as it is in the case of the Move-to-Front rule. Indeed, the Transposition rule could be defined as the Move-Ahead-1 scheme, and the Move-to-Front rule could be 


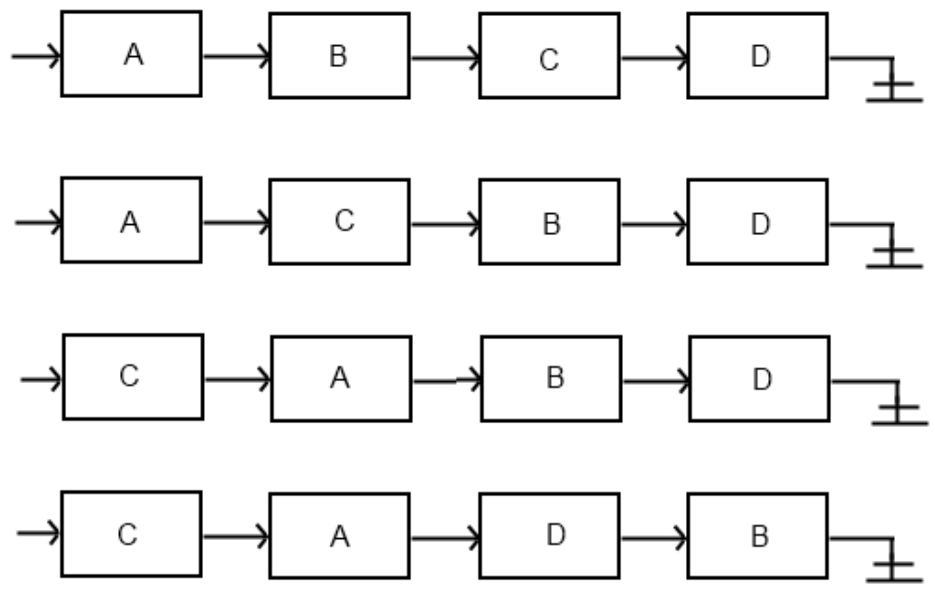

Figure 2.7: Execution of the Transposition update rule for the singly-linked list where the order of accesses is $C, C, D$.

defined as Move-Ahead- $n$, where $n$ is the length of the list [21].

The Move-Ahead- $k$ rule has been found, via empirical evidence, to converge faster when $k$ is larger, but, generally, at the expense of a greater asymptotic cost. This, clearly, parallels the observations of the Move-to-Front and Transposition rules [21]. Although the Move-Ahead- $k$ was defined in terms of singly-linked lists, an extension to doubly-linked lists similar to Move-to-End is both intuitive and natural.

\subsubsection{POS $(\mathrm{k})$}

The $\operatorname{POS}(k)$ updating mechanism is yet another method which balances between the extremes of the Move-to-Front and Transposition rules, although it does so in a more complex and, arguably, sophisticated fashion than the Move-Ahead- $k$ [21]. It has been proposed that, as a data structure approaches convergence, it would be best to switch from a Move-to-Front paradigm to a Transposition scheme. The issue here is 
that it is not easy to infer when convergence has occurred, and a series of queries can always be constructed that will "fool" attempts to do so with estimation [21]. The $\operatorname{POS}(k)$ mechanism was therefore developed to hybridize and balance between the two.

Under the $\operatorname{POS}(k)$ rule, for a value of $k$ such that $1 \leq k \leq n$, where $n$ is the length of the list, if an object in a position less than $k$ is accessed, it is transposed with the element ahead of it in the list, identically to what the Transposition rule does [33]. However, if the object is in a position greater than $k$, it is instead brought to position $k$, thus behaving like the Move-to-Front rule, where position $k$ is treated as the front of the list. The reader should again observe that we can express the Transposition and the Move-to-Front rules in terms of the $\operatorname{POS}(k)$ since the former is the same as $\operatorname{POS}(n)$, and the Move-to-Front rule can be expressed as $\operatorname{POS}(1)$.

\subsubsection{Stochastic Variants}

All of the ADS updating mechanisms for linked lists discussed until now, have been deterministic. However, it is certainly possible to consider update mechanisms that probabilistically reorganize the list when an element is accessed. As a trivial example, the Move-to-Front rule could be adapted so that the accessed element is only moved to the front $50 \%$ of the time (although this rule is strictly worse than deterministic Move-to-Front [34]). A more practical example, introduced by Oommen [32], involves moving the element to the front with a decreasing probability, which has the advantage of changing the ADS from being an ergodic system to an absorbing system. However, even in this case, the deterministic Move-to-Front rule performs better, and the stochastic version is at best expedient [32].

In the same work, Oommen introduced an asymptotically optimal stochastic Move-to-Rear updating mechanism [32]. In this version, accessed elements are moved to the rear of the list with a probability that reduces based on the number of times 
the element has been accessed [32]. Thus, when the list converges, as the probability of moving each element approaches zero over time, it is an absorbing system. Consequently, the elements accessed more frequently are likely to be located near the front of the list. This mechanism has the advantage in that the probability of convergence to the optimal arrangement is configurable, and it can thus be shown to be asymptotically optimal [32].

\subsubsection{Tree-Based ADSs}

Aside from techniques involving lists, ADS update mechanisms also exist for Binary Search Trees (BSTs). These are based on the same principle of seeking to reduce the overall average access times. However, in the case of trees, rather than bringing frequently accessed elements to the head of a list, update mechanisms seek to bring them closer to the root. This is done through the well-known rotation operation, performed iteratively on a queried element so as to move it towards the root $[3,15]$. As with lists, a range of update mechanisms exists for BSTs. While we do not employ BSTs in our work, we will briefly detail here some of the more well-known adaptive tree strategies ${ }^{3}$.

The analogous strategy to the Move-to-Front rule in the domain of BSTs, is, as one could expect, the Move-to-Root rule. As its name implies, the Move-to-Root rule iteratively performs the rotation operation on a queried element until it is the new root of the tree $[3,15]$. Unlike the Move-to-Front, where moving an element to the head of the list is an $O(1)$ operation, Move-to-Root requires iterative applications of an operation. Furthermore, it can compromise the balanced nature of a tree, and thus, this update mechanism is more costly in BSTs. However, as with the Move-toFront rule, the Move-to-Root offers a lower bound on expected search time, within a constant factor of the optimal tree [15].

\footnotetext{
${ }^{3}$ We record them here because all the list-oriented game playing strategies that we introduce in the subsequent chapters can be also designed using tree-based ADSs. Research along these lines is currently open.
} 
Analogous to the Transposition rule is the Simple Exchange update mechanism, where a single rotation operation is made on the queried element, moving it to its parent's position in the tree, and thus, towards the root [15]. This is advantageous in the domain of BSTs, as this update mechanism, due to it involving only a single rotation, is $O(1)$ in nature, compared to the Move-to-Root scheme. However, as with the Transposition rule, the Simple Exchange rule cannot guarantee the final performance within any constant factor of the tree's optimal configuration [3]. Additionally, while the Transposition rule can offer better asymptotic performance in many cases than the Move-to-Front rule, the Simple Exchange rule has been found to behave poorly even against queries drawn independently from a probability distribution, even when they are not constructed to challenge the data structure $[2,3]$.

Another ADS strategy for binary trees, is the well-known Splay Tree, which replaces normal tree mechanisms with several special operations, and includes the Moveto-Root rule in its basic construction [2]. All standard binary tree operations are accomplished through "splaying", a special process involving three possible operations, which brings the accessed element to the root of the tree [35]. Splay Trees are a well-known variety of data structures that fall outside the area of ADSs. As we will not employ them further in our work, we will not describe them here, in any detail. Many variants of Splay Trees that have been introduced, such as the Tango Tree, known for offering a very tight bound, in comparison to the optimal tree [14, 70].

A number of more complex update mechanisms for BSTs exist, such as the concept of Conditional Rotations. In the case of a BST employing the Conditional Rotation rule, additional information is stored within each node, including the number of times a node has been accessed, and its path length. Collectively, these are employed to determine when the accessed node should be brought closer towards the root and how far it should be moved, so as to reduce the costs of the Move-to-Root rule [10]. We omit more detailed descriptions of these tree-based ADSs as they are not currently pertinent to our work. 


\subsection{Chapter Summary}

In this chapter, we have provided a survey of the fields of game playing, including techniques from both two-player, and multi-player strategies, as well as the techniques from the formerly-unrelated field of ADSs. Given the enormous breadth of each of these fields, we have focused our discussion on those techniques that we will be incorporating in this research endeavour. For the rest of this work, we will describe, and experimentally demonstrate, how ADS-based techniques can be employed to improve intelligent two-player and multi-player game playing strategies. 


\section{Chapter 3}

\section{Improving the Best-Reply Search using ADS}

\subsection{Chapter Overview}

In the previous chapter, we introduced the current techniques for playing two-player and multi-player games, considered to be the state-of-the-art. We also discussed the field of ADSs, which provides the inspiration for the techniques that we develop to improve on game-playing strategies. This chapter ${ }^{1}$ represents the first of our efforts to merge these two fields, by combining techniques derived from list-based ADSs with existing multi-player techniques. We first review and elaborate on move-ordering heuristics for deterministic game playing algorithms, and then showcase the methods by which they can improve on the benefits of alpha-beta pruning, which we earlier discussed in Section 2.4.3. We then introduce the concept of relative opponent threat levels, and develop methods by which they can improve move ordering in a multiplayer game. To the best of our knowledge, this approach has been unexplored in the literature.

\footnotetext{
$[43]$.

${ }^{1}$ The results in this chapter were originally presented in [37] and [40], and in the journal paper
} 
Given that the list-based ADS techniques, discussed in Chapter 2, are capable of moving frequently accessed data objects to the head of the list, we provide a rationale for using an ADS to rank opponents, in the order of the threat they pose to the perspective player. We describe how the salient features of list-based ADSs both enable them to perform this task intuitively, and how this can be accomplished inexpensively. We then propose the Threat-ADS heuristic, which is a move ordering heuristic making use of these principles for the Best-Reply Search (BRS) (introduced in Chapter 2 and detailed in this chapter) that can achieve statistically significant improvements in tree pruning with minimal cost.

\subsection{Move Ordering}

The concept of move ordering, within the context of Mini-Max style game playing, was discussed in Chapter 2, and specifically in Section 2.4.3. Also presented in that chapter was a collection of well-known and proven strategies for improving move ordering in game trees during exploration, which have seen significant analysis in research over the years. As it is particularly relevant to the work presented in this chapter, we will now expand and build on the information previously presented.

\subsubsection{Benefits of Move Ordering}

When we speak of move ordering in game trees, what we refer to is the order of the children of a node, from its leftmost to its rightmost child. When the game tree is explored in a depth-first manner, as in the Mini-Max algorithm, this, consequently, determines the order in which the children are visited. A node in a game tree represents a specific board state, including the player whose turn it is to make a move. Its children represent the resultant board state from each of the player's available moves. As in the reported literature, we will refer to the leftmost child as the "first" move, proceeding to the rightmost child as the "last" move, modeling the children as a list. 
When considering an entire game tree, either a complete tree from the start to the terminal states, or until some cutoff point, the order of the moves is not relevant. The Mini-Max algorithm will return the same final value regardless of the order in which the moves are considered. Move ordering becomes important, however, when tree pruning is employed. This is the case in virtually all practical systems of this sort, given its substantial benefits.

The alpha-beta algorithm (Algorithm 2.1) produces cutoffs when a move is found to have no possibility of influencing the final value of the game tree. It can accomplish this most efficiently, achieving its maximum possible tree pruning, when the best move at each node is always considered first. Therefore, move ordering within the context of the alpha-beta paradigm attempts to achieve this arrangement. Of course, in a real-world game, achieving this perfect move ordering is impossible, because of the fact that, to construct it, one would need to have a perfect knowledge of the game tree and the opponents' strategy. This problem has led to researchers investing significant time and effort in the analysis and development of novel move ordering strategies, in an attempt to develop heuristics to better order the moves. The History and Killer Moves heuristics, discussed in Section 2.4.3.1, are examples of two such techniques.

\subsubsection{Examples of Move Ordering}

To showcase the benefits of move ordering, consider the fragment of an alpha-beta tree in Figure 3.1. We see that an alpha cutoff has occurred on the second level, in the rightmost child, and we further know that it cannot influence the final value of the tree. Thus, we do not need to consider any more of its children, and by pruning at this juncture, the consequent node expansions have been saved.

Observe that, in this example, we would have already examined several of the node's children, before we were able to determine that it could not influence the value of the tree. Consider Figure 3.2, in which the same cutoff is generated by the same child node, but when this occurs much earlier because the powerful move has 


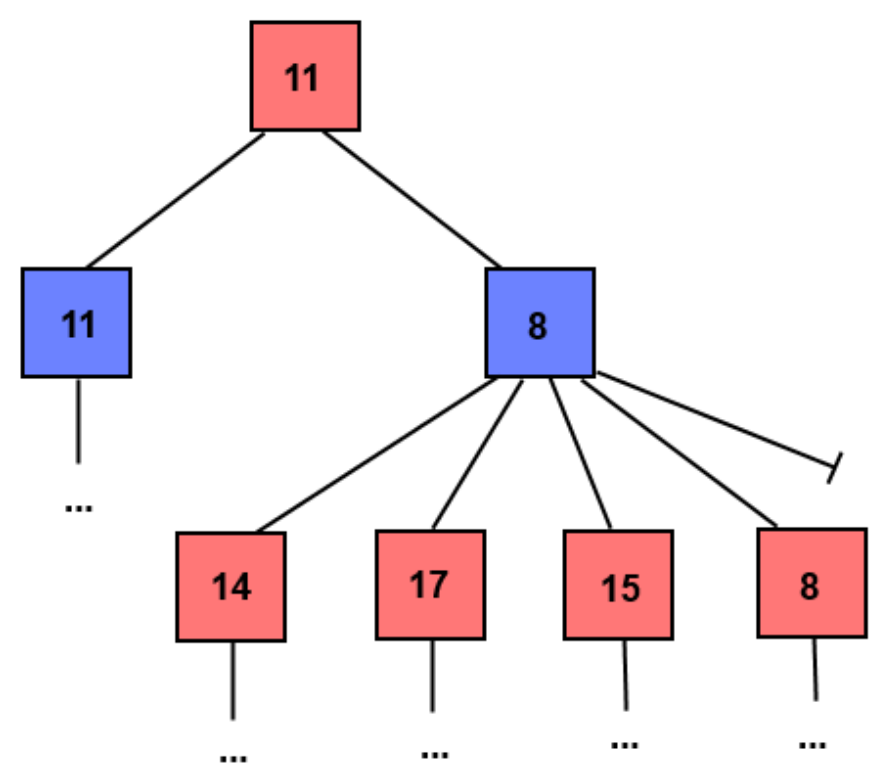

Figure 3.1: An example of alpha cutoff when the move ordering is poor.

been examined first. This can represent a substantial savings in terms of search time, because if these nodes are higher in the tree, each of the nodes eliminated could have thousands or even millions of descendent nodes. We can thus see the potency of move ordering.

There are many possible move ordering heuristics and techniques reported in the literature. As a simple example, consider the case of Chess. Moves that capture pieces are often better than those that do not, and so a simple move ordering heuristic for Chess would be to consider these "capturing" moves first. This, however, is a example of a domain dependent heuristic. While it may generate strong results within the context of Chess, it does not apply to any other games. Domain independent move ordering techniques tend to require more thought and intuition, and some well-known examples have been discussed in Section 2.4.3 and in its subsequent sections. 


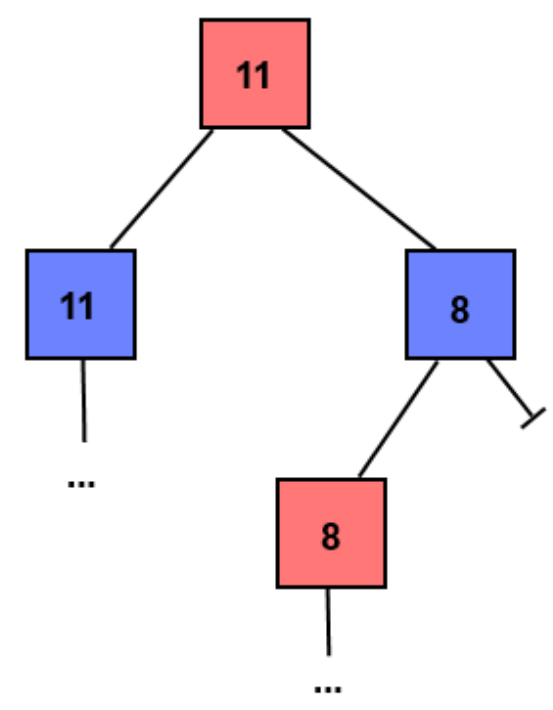

Figure 3.2: An example of alpha cutoff when the move ordering is planned efficiently.

\subsection{Opponent Threat Level}

We now discuss the concept of the so-called opponent threat, which will be related to move ordering in the work presented in this chapter. When we refer to the "threat" posed by an opponent, we are speaking of the degree to which that opponent can minimize the perspective player, either by damaging his position within the game, or by winning the game, and thus posing an obstacle to the perspective player winning the game. We will refer to this degree of threat as the "threat level" of the opponent. Specifically, however, we are interested in the relative opponent threat levels when developing our new strategies here. In other words, we consider how the threat levels of different opponents, in a multi-player context, compare to one another, and the inference of a ranking of the opponents, from the most to the least threatening. 


\subsubsection{Properties of Opponent Threat}

Determining how threatening a given opponent is in a multi-player game, is a phenomenon that is familiar within the field of game playing. As an example, the Softmax-N algorithm, described in Chapter 2, makes an effort to determine how good a particular opponent's strategy is, which translates directly into quantifying the threat that the opponent poses, so as to appropriately achieve opponent modeling $^{2}$. Furthermore, learning how an opponent plays and how effective his strategies are is a core concept in playing the game Poker [31].

When attempting to determine the threat that a specific opponent poses to the perspective player, there are several metrics that can be considered. When we are contemplating the issue of opponent modeling, we are interested in analyzing the opponent's previous decisions. This will help us to construct a model or philosophy of what he is likely to do in the future. In this way, we can also identify intelligent opponents, or those that frequently make effective decisions, and they can be classified as being those that pose a greater threat, relative or otherwise. Another interpretation, which appears less frequently in the literature, is to consider, in a board-based multi-player game, who is the opponent in the most threatening position. We will be making use of this interpretation within the work presented in this chapter.

As an example, consider the layout of the Virus Game (the rules for which are included in Appendix A) shown in Figure 3.3. As players in the Virus Game may "infect" adjacent squares, we can clearly see that the Blue opponent has more moves available to him that minimize the Red player, than the others. Thus, we can determine that the Blue player poses the greatest relative threat.

In this case, it is obvious which opponent is the most threatening. In a more complicated game, it is not always as easy to guess the relative threat of each opponent, as we will showcase below.

\footnotetext{
${ }^{2}$ The details of this paradigm are referred to in Section 2.5.3.1, but are not included here to avoid repetition.
} 


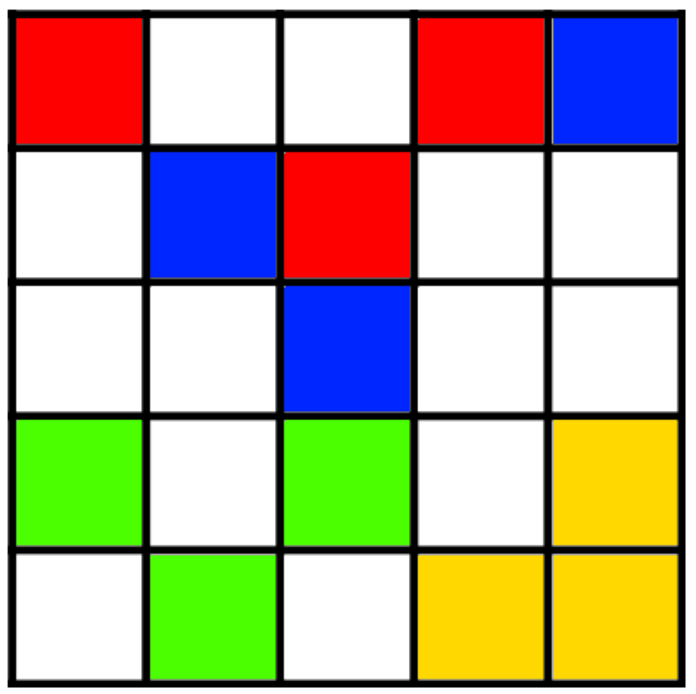

Figure 3.3: An example layout of the Virus Game with a threatening opponent.

\subsubsection{Opponent Threat and Move Ordering}

Observe that there is a parallel between move ordering and relative opponent threat levels. In both cases, we wish to construct a ranking, either of the best to worst move, or the most threatening to the least threatening opponent, both of which can be appropriately modeled as lists. We therefore consider the possibility that these concepts can be combined in some beneficial manner, which is an idea that, to the best of our knowledge, has not been utilized or implemented in the literature until now. We shall now introduce the basis by which this may be accomplished, and will thereafter proceed to show how it can be implemented.

Although both concepts make use of a list-based ranking, there is no direct relation between opponent threat ordering and the corresponding move ordering. In the normal manner of play, each opponent acts in a pre-defined order, and their possible 
moves are collected and considered individually. The long-standing Paranoid and Max- $\mathrm{N}$ algorithms operate in this way, where each opponents' moves are considered on his own level of the game tree. In this case, there is no easy or straightforward way of relating relative opponents' threats and move orderings. However, when one considers the far more recent BRS, we perceive a case where these concepts can be related quite naturally.

Our strategy is motivated by the fact that the BRS transforms a multi-player game tree into a two-player game tree by grouping opponents together into a single "superopponent". At each minimizing level, we consider what moves could be made by each opponent, allowing each of them to act out-of-turn if required, and thus grouping these moves together. From this perspective, we see how one can link opponent threat levels to move ordering.

More threatening opponents, in terms of the current board position, are more likely to produce moves leading to a cut, if their moves are grouped with other opponents, as they can potentially minimize the perspective player more. Therefore, if we consider these opponents' moves first, we anticipate that better move ordering can be achieved, within the context of the BRS. As an example, consider Figure 3.4, which shows the execution of a single minimizing level of the BRS. Note that the move selected, which is highlighted in red, provides the value of the tree at this step.

The reader will notice how the fourth player's move is the strongest, and it provides the value of the tree at this node. Thus, it would have been expedient if we could have considered it earlier, if possible. Now, consider Figure 3.5, in which the relative opponent threat levels are known, and provided in the list at the bottom of the image. In this case, the strongest move is much closer to the leftmost child. Since these moves allow more pruning to occur, this will result in a more efficient search.

This argument shows that knowledge of the relative opponent threat levels can lead to improvements in tree pruning within the BRS. Our intention is to see if we can use this knowledge to provide new heuristics, which not only will make use of opponent threat levels (a phenomenon that has not been combined with move ordering 


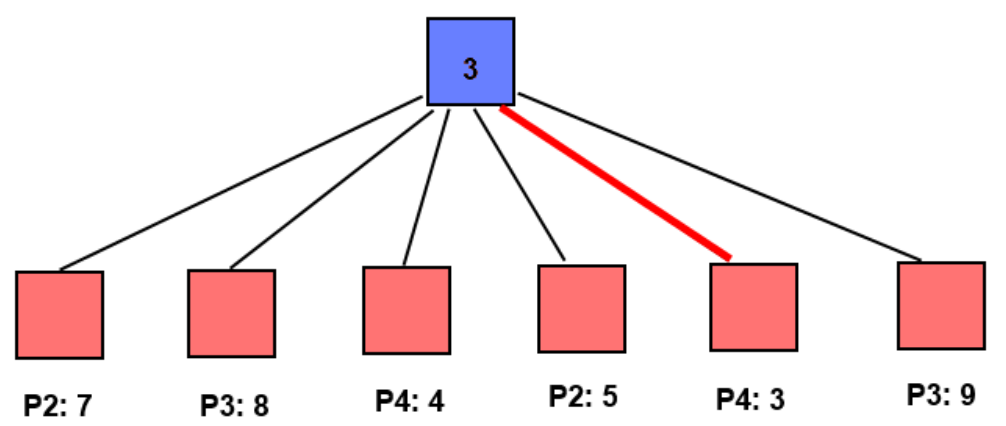

Figure 3.4: The subtree associated with the BRS without considering the various opponents' threat levels.

before), but to also use features specific to a multi-player context. The use of qualities specific to a multi-player context to achieve move ordering is also a concept that, to the best of our knowledge, has not been explored within the literature. The question that we now encounter is the following: How does one acquire the relative opponent threat levels? Clearly, we must, somehow, learn them from the game state in order to get any benefit from them. We will now describe how this can be accomplished, using techniques borrowed from ADSs.

\subsection{Managing Opponent Threat Level Using an ADS}

In the case of the instance of the Virus Game shown in Figure 3.3, it is apparent from visually inspecting the board state that the Blue player has the potential to make 


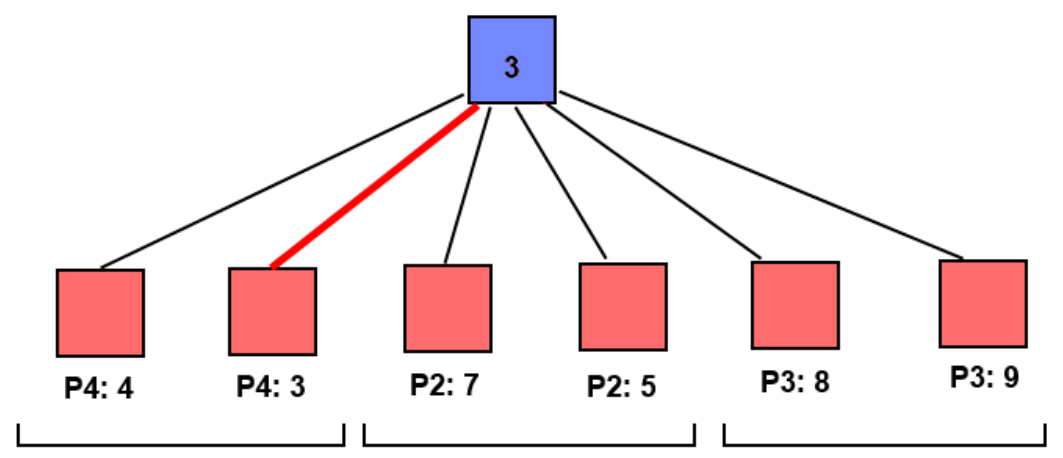

\begin{tabular}{l|l|l|l|} 
Threat Order: & P4 & P2 & P3 \\
\hline
\end{tabular}

Figure 3.5: The subtree associated with the BRS when it is guided by the threat levels of the opponents.

more moves that can minimize the Red player than the other opponents, and thus he poses the greatest threat to the Red player. It is not always the case, however, that such an inference is so readily apparent. Consider, for example, the Focus (the rules for which are included in Appendix A) game shown in Figure 3.6, where the numbers indicate the height of each stack. In this case, one sees that the Yellow player mostly surrounds the perspective player, and one may assume that he poses the greatest threat, as a result. However, the Blue player has two larger stacks that, while further away, due to the rules of Focus, could be used to capture a significant number of the Red player's pieces in a single move, up to a total of four. Thus, we see a situation where a simple heuristic to determine which opponent is the most threatening meets with failure.

Following this failure, one might propose an alternative means of estimating opponent threat levels for Focus, where the presence of large stacks could highlight a 


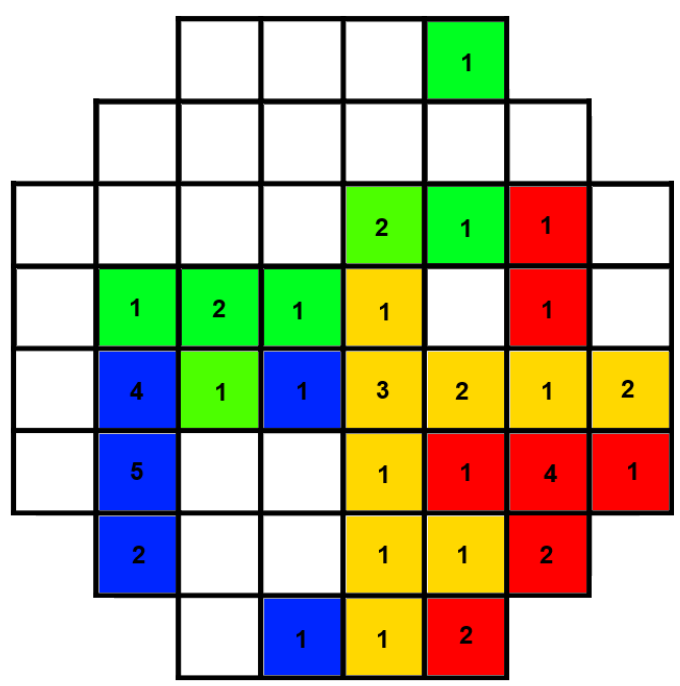

Figure 3.6: An instance of the Focus game showcasing opponent threat levels. The numbers represent the heights of the stacks.

more threatening opponent, which is potentially true. However, while one could certainly construct highly accurate heuristics to rank opponents in terms of threat for specific games, making use of the knowledge of the game's rules and how it is played, one can glean an advantage if a ranking technique could be discovered that works for multiple games. Techniques in this category, which we shall refer to as domain independent techniques, offer considerable value, as they can be applied to a broad set of games without the need for significant redesign. Examples of domain independent techniques include the Killer Moves move ordering technique, discussed in Chapter 2, and the alpha-beta algorithm itself.

As we are interested in making use of threat levels to improve move ordering, we recognize that the system we use to estimate opponent threat levels must be capable of accomplishing this task quickly, and with limited resources. Otherwise, the anticipated savings from improved move ordering may be nullified by the resources spent in learning the threat levels. Furthermore, the opponent threat levels due to 
board positions may change over the course of the game, as opposed to a static concept of threat, such as the opponent's skill. Thus, it is not feasible for us to make use of training techniques that learn how threatening opponents are over a period of time, and rely on this training in the future, as is the case of the Softmax-N algorithm. Furthermore, a technique that can accomplish this in a domain independent manner would offer considerably more value, if it is possible to develop one.

Fortunately, as it turns out, techniques used within ADSs provide exactly the sort of properties we desire. It is here, then, that we can forsee how the fields of game playing and ADSs can be combined. We propose to use a lightweight, listbased ADS to rank our opponents by their relative threat levels. To do this, we will place each of the opponents within an adaptive list (one of those introduced in Chapter 2.7), and employ an update mechanism to allow the ADS to "learn" the relative opponent threat levels. As the structure is a list, the relative threats will be represented naturally, by each opponent's position within the list, from the most to least threatening, at any given point.

An example of how this could work, again using the Virus Game example from Figure 3.3, is outlined in Figure 3.7. In this case, we are presented with the ADS, and the Blue player, through some mechanism, is found to be the most threatening at this snapshot of the game. Therefore, we query the ADS with his identity (here represented as "P2"), and the ADS adjusts its structure as if an element was accessed, moving this opponent to the head of the list. We have thus updated the ranking of each opponent, and can thus leverage this information elsewhere in the tree, for move ordering or, for that matter, for any other purpose that we envisage. 


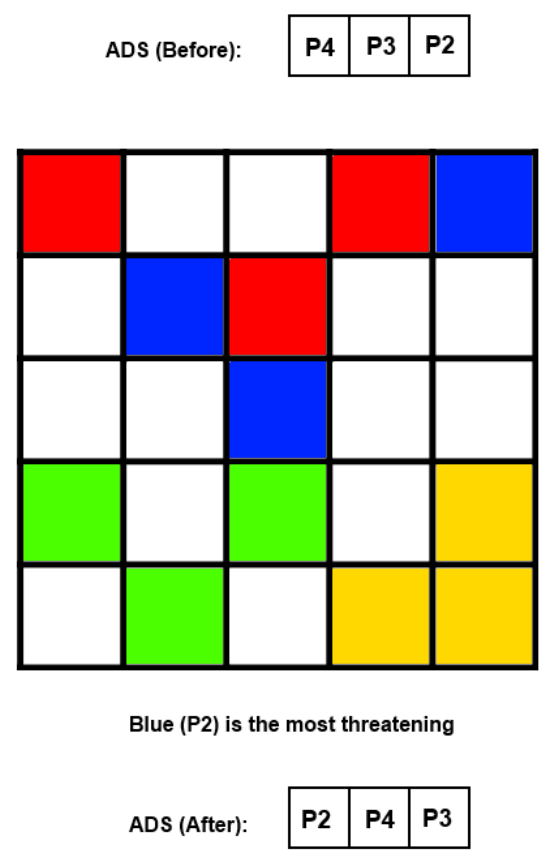

Figure 3.7: A demonstration of how an ADS can be used to learn relative opponent threat levels over time (P1: Red, P2: Blue, P3: Green, P4: Yellow).

This argument demonstrates how the list-based ADS can be used to model relative opponent threats. However it leaves us with an obvious open question: "By what mechanism was the Blue player found the be the most threatening?" A domain dependent technique, which analyzes the board state and concludes that the Blue player has the greatest potential to minimize the perspective player, could provide this mechanism. However, a domain independent technique would be much more valuable in terms of reusability. The answer to this problem will be provided in the next section, within the context of the BRS, along with a complete move ordering heuristic that makes use of it. We have called this strategy the Threat-ADS heuristic. 


\subsection{The Threat-ADS Heuristic}

In the preceding sections, we described all of the pieces required to develop our new move ordering heuristic. The BRS will provide the game playing framework, and we wish to augment its move ordering using relative opponent threat levels. We will accomplish this via storing representations of the opponents within the ADS, where its structure represents a ranking of each opponent, in terms of their respective threats. We will use this representation to improve move ordering in what we consider to be a very intuitive fashion. The remaining unknown element, which we will outline below, is that of determining how and when the ADS can be updated.

\subsubsection{Developing the Threat-ADS Heuristic}

Consider the execution of the BRS, described in detail in Section 2.5.4. We observe that, at each "Min" phase, the BRS determines which opponent has the most minimizing move. The phenomenon of having the most minimizing move against the perspective player, and also being characterized as having the highest relative threat level against that player, are conceptually linked. With that in mind, we propose querying the ADS with the opponent that is seen to possess the most minimizing move during a Min phase, advancing his position in the relative threat ranking, thus allowing the ADS to learn a complete ranking over time.

The next issue that needs to be addressed is that of deciding the Min phases at which we will query the ADS. For the purpose of this chapter, we opt to make the decision to query the ADS with the most threatening opponent at each Min phase of the algorithm, and to correspondingly maintain a single list-based ADS. Alternative schemes will be explored in more detail, along with their benefits and drawbacks, later in this work. To accomplish the move ordering itself, we will simply explore each opponent's moves in the order dictated by the ADS, from the most to the least threatening. 
The salient feature of the Threat-ADS heuristic is the following: At each Min phase, we explore the relevant moves in the order from the most to the least threatening opponent, and then update the ADS by querying the opponent who was determined to have the most minimizing move overall. Algorithm 3.1 shows the complete, formal BRS algorithm, making use of the Threat-ADS heuristic.

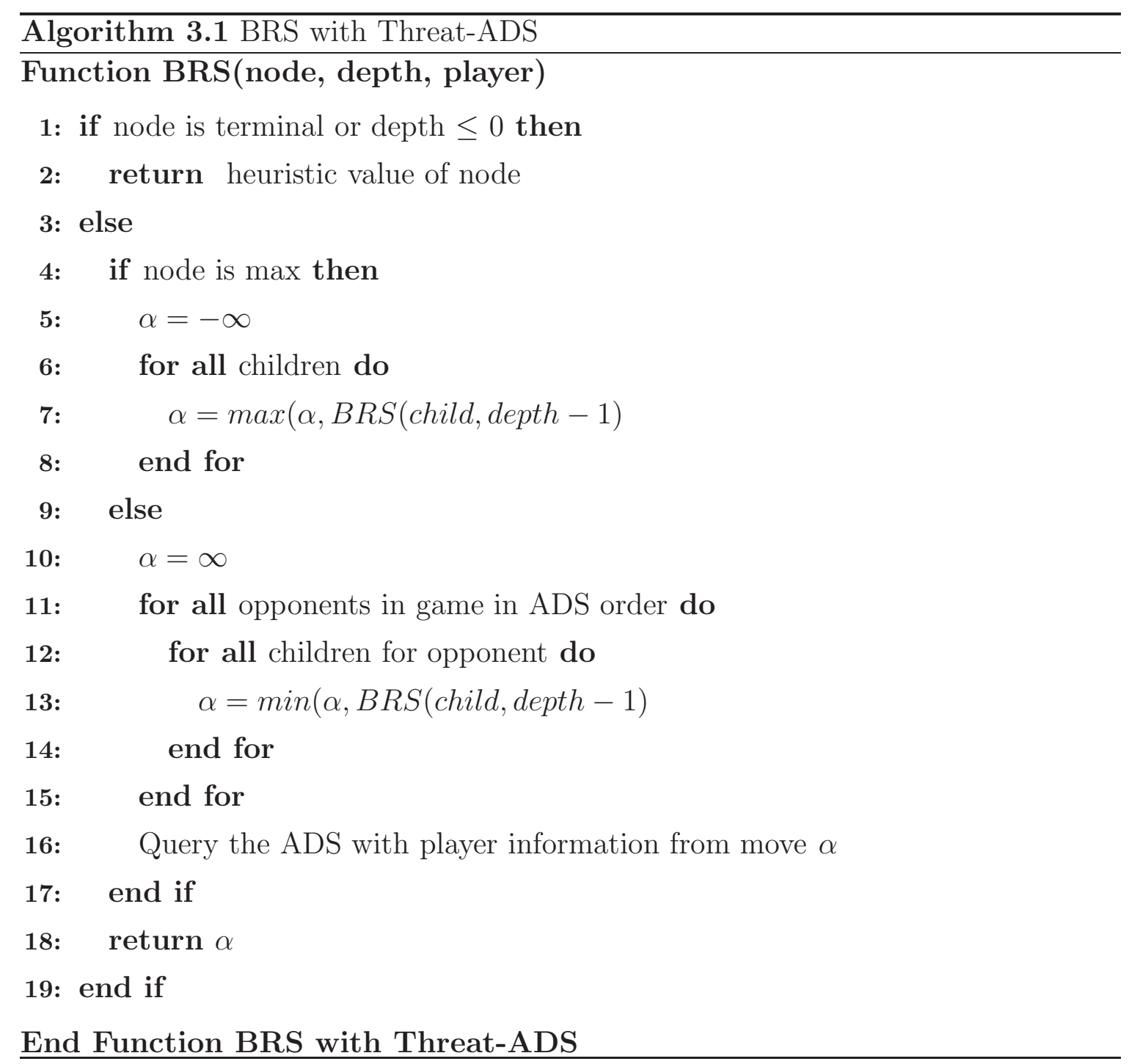

To demonstrate visually the execution of the Threat-ADS heuristic, we have included an example in Figure 3.8. This figure shows how the ADS is updated, based on the identity of the opponent who was found to have the most minimizing move, 
at a certain level of the tree. In our specific example, opponent "P4" has the most minimizing move, and thus the ADS is updated by moving him to the head of the list. Notice that this update is domain independent as opposed to the domain dependent one (i.e., for Virus) in Figure 3.7.

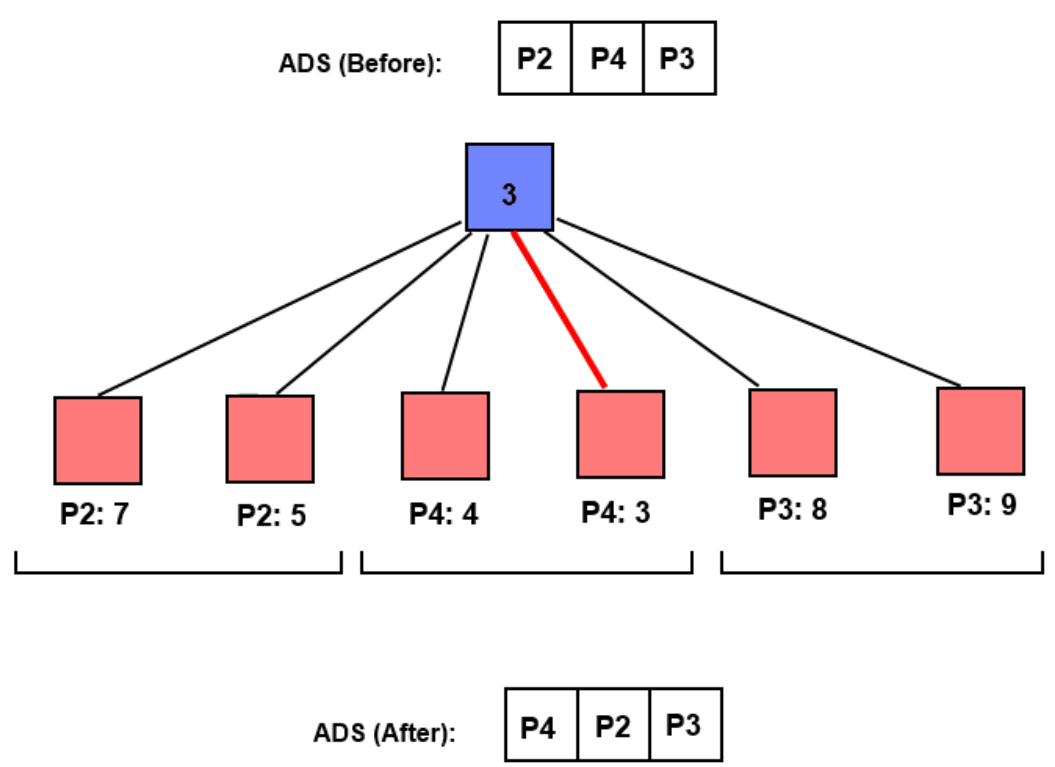

Figure 3.8: A demonstration of how the Threat-ADS heuristic operates over time.

\subsubsection{Salient Features of the Threat-ADS Heuristic}

We first highlight a number of qualities possessed by the Threat-ADS heuristic, and its operating mechanisms. First of all, we observe that, as is common with moveordering heuristics, the use of the Threat-ADS heuristic does not in any way alter the value of the tree. Consequently, there can be no destructive influence on the decision making process that the BRS undergoes. 
More importantly, although the BRS is subject to traditional move ordering techniques, it does not impose any natural ordering on the opponents or how their moves are collected. Therefore, no information can be lost when one uses the Threat-ADS heuristic to decide the order in which one collects moves from the opponent. It is possible that more complex heuristics, such as those making use of expert domain knowledge, could result in a greater reduction of the tree size than the lightweight Threat-ADS heuristic. However, if the developer has a particularly strong move ordering technique for his specific domain, the Threat-ADS heuristic can still be employed, to break ties, or as a lower-priority strategy. This is a common practice in modern game playing engines, such as those proposed in the original BRS system [52].

The Threat-ADS heuristic adds the adaptive linked list to the BRS's memory footprint. Luckily, the list only has a length equal to the number of opponents, which, outside of the unusual case of Population Games (discussed in Chapter 2) to which the BRS does not easily apply, is a very small constant or diminuting (in the case of eliminations) number, and is typically no more than seven.

We furthermore note that the vast majority of list-based ADSs feature very intuitive, constant-time update operations. This generally involves no more than moving the position of a single element within the linked list. This is, clearly, a very small expenditure when compared with other operations that must be completed at each Min node of the tree, such as collecting all possible moves for each opponent, and organizing them into a data structure for exploration. Perhaps more importantly, the Threat-ADS heuristic does not require the opponent moves to be sorted. While one could naively sort a collection of moves in terms of the opponents' positions in the ADS, in fact, we can simply generate moves for each opponent in the order of their occurrences within the ADS. This is in contrast to a number of very powerful heuristics, such as the History heuristic, where sorting of moves is required, and where this operation has been observed to negatively impact runtime [54].

From the above discussion, we thus conclude that invoking the Threat-ADS heuristic incurs a very low cost for the BRS. 


\subsection{Experimental Model}

The fact that the Threat-ADS heuristic can be applied to the BRS, and that its application incurs a very low cost in terms of memory and execution time, are now clear. Indeed, it has the potential to achieve a new kind of move ordering, yet unexplored in the literature, i.e., one that is based on domain independent multi-player specific qualities. However, in order to confirm that the use of the Threat-ADS is worthwhile, we must provide a demonstration and analysis of how it behaves in the context of real-world game trees. Given the extreme difficulties inherent in the formal analysis of game-playing algorithms (as has been discussed in great detail [63]), we, instead, choose to verify its performance via experimentation, as is the accepted practice in the field.

What we are interested in learning is the improvement gained when using ThreatADS, when compared to not using it, to a given search depth within a game tree. While the Threat-ADS is, as discussed above, a game-independent move ordering technique, if we were to only test it in the context of a single game, we would have no information regarding its performance in other games. Thus, we have decided to test it with the spectrum of games discussed in Appendix A, specifically the Virus Game, Chinese Checkers, and Focus. The reasons for this are the following: Chinese Checkers and Focus are chosen due to the fact that they are both acclaimed multiplayer board games, and their use has been confirmed in the paper that originally reported the BRS [52]. They are also contrasted from each other in terms of the objective of the game, as the goal in Chinese Checkers is to race one's opponents to the end state, whereas, in Focus, it is to capture opponent pieces.

We also choose to use the Virus Game, of our own creation, which is another piececapturing game with very different rules and possible moves from Focus, as well as its highly configurable nature, which will assist in running a breadth of experiments. In this context, the Virus game will be played on a 5 -by-5 board, with each player having three pieces in a starting configuration, and where each player will use the "cross" virulence. 
We recall that the Threat-ADS requires the use of an adaptive list to keep track of the relative opponent threat levels. However, when specified in the generality, it does not state the specific update mechanism that must be used. For the purposes of the work in this chapter, however, we will use the "Move-to-Front" update mechanism, discussed in Chapter 2.7, with the Threat-ADS heuristic. Exploration of alternative update mechanisms, as well as their impact on the Threat-ADS heuristic's performance, will be discussed in the next chapter.

Since the Threat-ADS heuristic may be able to retain its knowledge in subsequent turns, we will allow the game to proceed for several turns from the position where we initiate the analysis. Further, as the Threat-ADS does not influence the decisions of the BRS, but only its speed of execution, the actual performance of the BRS cannot be influenced if the ply depth is fixed. Thus, the end result of the game is not a fundamental concern in our experiments, and so, in our experimentation, we will not run the games to termination. Specifically, we will run the Virus Game for ten turns, Chinese Checkers for five, and Focus for three. These numbers are primarily chosen to reduce the execution time of the experiments. In this chapter, we will limit the search to 4-ply. The impact of using ADS-based strategies in trees of larger ply will be investigated later. Given a desire to decrease the running time of the experiments, and to address a broader range of possible board configurations, even those that could result from poor play decisions, opponent players acted randomly in between these turns.

It seems intuitive to use the CPU time as a measure for these experiments, as the purpose of tree pruning is to reduce the runtime of the Mini-Max algorithm. However, it has been observed in the literature that the CPU time is a problematic metric for experiments of these sorts. This is because it is prone to be influenced by the platform used and by the specific implementation [54]. We, therefore, follow the accepted litature and make use of the Node Count (NC) measure, introduced by Schaeffer, and which has been found to be highly correlated to an algorithm's runtime [54]. Formally, the NC measure is defined as the sum of internal and leaf nodes at which computation takes place. This excludes those nodes that were considered, but 
never visited, as an alpha or beta cutoff occurred before that point, at their ancestor. As the NC measure deals only with how the tree is grown, it is not subject to any platform-specific influences.

More specifically, we will take, as our final measure, the sum of the NC over the turns over which the game is played. While the intial experiments reported in [37] were each run two hundred times, we found that this was computationally burdensome and that it also led to a unnecessarily high level of statistical certainty for our purposes. Consequently, in this chapter, so we have limited the work to fifty trials, to save time and allow us to broaden our analysis of the Threat-ADS heuristic, and to permit us to make use of a larger ensemble of experimental settings.

All our experiments were run on an Intel i5 $3.4 \mathrm{GHz}$ processor (a four core machine). Although we have elected to not employ runtime as our metric for comparison, a brief discussion of the running time of the experiments is presented at the end of this chapter, alongside a discussion of our results.

\subsection{Prima Facie Experiments}

To consider the scenarios initially discussed in [37], we will first run the games from the starting position, with four players for the Virus Game and Focus, and six for Chinese Checkers. Subsequent work in this chapter will vary these parameters to obtain a better understanding of its performance within different environments. Table 3.1 showcases the results of the Threat-ADS heuristic ${ }^{3}$, within the context of these settings. As is clear from the table, there is a reduction in computation, or improvement in the efficiency, in all cases, when the Threat-ADS is used alongside the BRS. These results are roughly equivalent to those we first presented in [37], however the degree of improvement is noticably different, with a $6 \%$ reduction in tree size for Focus, and

\footnotetext{
${ }^{3}$ As recommended by the members of Committee evaluating the Thesis proposal, wherever appropriate, all the figures cited in the tables and experimental results, have been rounded to the closest hundred. We are grateful to the members of the Committee for suggesting this.
} 
a $10 \%$ and $12 \%$ reduction for the Virus Game and Chinese Checkers, respectively. For example, in the case of the Virus Game, the NC was reduced from 264, 200 to 237,000 .

Table 3.1: Results of the Threat-ADS for the various Game Models

\begin{tabular}{|c||c||c||c||c||c||c|}
\hline \hline Game & ADS? & Avg. NC & Imp. & Std. Dev. & P-Value & Effect \\
\hline Virus Game & No & 264,200 & - & 32,400 & $3.0 \times 10^{-5}$ & 0.84 \\
Virus Game & Yes & 237,000 & $10 \%$ & 29,100 & $3.0 \times 10^{-5}$ & 0.84 \\
\hline Focus & No & $6,859,100$ & - & 685,300 & $1.9 \times 10^{-3}$ & 0.61 \\
Focus & Yes & $6,443,900$ & $6 \%$ & 805,300 & $1.9 \times 10^{-3}$ & 0.61 \\
\hline C. Checkers & No & $3,485,400$ & - & 850,300 & 0.014 & 0.49 \\
C. Checkers & Yes & $3,070,400$ & $12 \%$ & 836,600 & 0.014 & 0.49 \\
\hline
\end{tabular}

While we see a visible improvement in all cases, it is necessary to employ some form of statistical analysis on the results, to insure that they are significant and not merely due to "chance". In the case of the results obtained in [37], where we utilized an ensemble of 200 experiments, we found that under the Shapiro-Wilk test for normalcy, all the results were likely obtained from a normal distribution. However, our results here present a more general perspective, and the results for Focus, when the Threat-ADS was employed, did not pass the test for normalcy. Furthermore, we observe in this work that failing to reject the null hypothesis that the data is normal, as is the case under Shapiro-Wilk, does not necessarily allow one to conclude that it actually is normal. Therefore, rather than use the parametric $t$-test, as in [37], we instead use the non-parametric Mann-Whitney test for statistical significance in the present experiments. We find that in all cases, we are able to reject the null hypothesis with at least $95 \%$ certainty, showing that the Threat-ADS is producing a statistically significant improvement in all the cases analyzed. The results are thus consistent, and constitute an enhanced version of those reported in [37].

Also included in Table 3.1 is the Effect Size measure, which is simply the standardized mean difference between the two datasets (Cohen's $d$ ) [11]. We have included the Effect Size because, while the $P$-Value indicates the certainty that the difference 
between the datasets is significant, it does not indicate how strong this difference is $[11,69]$. We note that an Effect Size of 0.5 is considered "medium" and one of 0.8 is considered "large" [11], suggesting that the difference is relatively strong.

\subsection{Variation on the Number of Players}

As the Threat-ADS operates by ranking opponents within an adaptive list, and by deriving move ordering from that list, a potential impact on its performance could be the length of the list. This directly translates into the number of opponents present, or the number of players in the game. Therefore, it is of interest to us to vary the number of players in the games, to see how the performence of the Threat-ADS is affected.

In the case of the Virus Game, any number of players can be assigned to play the game, from two to an uncapped number. For Focus, the rules have been specified only for games involving two, three, and four players. Chinese Checkers can handle any number from two to six players with the exception of five. The reason why five players is omitted is because it would afford one player, the one with no opponent across from him, an unfair advantage (the details of this are given in Appendix A). However, as we are not running the game to completion, we have decided to test the five player case as well, with two configurations. The first case is the one in which the perspective player has no opponent across from him, and second is the case where there is no opponent adjacent to him. We hypothesize that the Threat-ADS will have an easier time producing results in the second case, as only one of the four opponents can easily minimize the player at the start of the game. The two player case is of no interest to us, and so we will only run tests with three or more players.

Results for these trials are presented in the next section. Where applicable, results from the previous set of experiments are repeated, to provide consistency across the tables and to allow an easy comparison. The same statistical tests as those posited earlier were performed on the results of these experiments. Given that our previous 
results did not pass checks for normalcy, we do not make the assumption of normalcy when analyzing these results as well. Therefore, we employed the Mann-Whitney test to test for statistical significance in all cases, and the Effect Size is provided, as previously.

\subsubsection{Results}

Table 3.2 shows the results of the experiments when three players are in the game. The Virus Game and Focus each return improvements comparable to those found in the previous section, and in [37], with an $8 \%$ and $6 \%$ reduction in tree size. For example, in the case of the Virus Game, the reduction is from 219, 100 to 201,700. The $P$-Values and Effect Sizes for the Virus Game and Focus are comparable to those in the original experiments, although the Virus Game has a slightly reduced Effect Size. Chinese Checkers saw no improvement at all, and the resultant average when the Threat-ADS is applied is actually slightly higher. This change is furthermore not statistically significant, and has a very low Effect Size. However, given that the players are isolated from each other at the start of a three-player Chinese Checkers game, it matches expectations that the Threat-ADS would not produce improvements, as no opponents can possibly minimize the perspective player.

Table 3.2: Results by Game Model - Three Players

\begin{tabular}{|c||c||c||c||c||c||c|}
\hline \hline Game & ADS? & Avg. NC & Imp. & Std. Dev. & P-Value & Effect \\
\hline Virus Game & No & 219,100 & - & 34,500 & $1.5 \times 10^{-4}$ & 0.50 \\
Virus Game & Yes & 201,700 & $8 \%$ & 25,400 & $1.5 \times 10^{-4}$ & 0.50 \\
\hline Focus & No & $5,290,200$ & - & 499,100 & $1.5 \times 10^{-3}$ & 0.60 \\
Focus & Yes & $4,988,400$ & $6 \%$ & 435,000 & $1.5 \times 10^{-3}$ & 0.60 \\
\hline Chinese Checkers & No & 732,400 & - & 181,700 & 0.18 & 0.07 \\
Chinese Checkers & Yes & 744,900 & $-1.5 \%$ & 148,200 & 0.18 & 0.07 \\
\hline
\end{tabular}

Table 3.3 reports our results when four players are involved in each game. The results for the Virus Game and Focus are the same as those in the previous section. 
Chinese Checkers, however, sees the best performance, with a slightly larger $14 \%$ reduction in tree size in the four player case (a reduction from 1, 380, 900 to 1,191, 700). It is quite likely that this is because only a single opponent is adjacent to the perspective player in a four-player game of Chinese Checkers, and that he is the only one who poses a threat. Therefore, we suspect that Threat-ADS is correctly prioritizing this player's moves in the search. Each of the changes is statistically significant, and the respective Effect Sizes are identical to or very similar to those presented in the previous section.

Table 3.3: Results by Game Model - Four Players

\begin{tabular}{|c||c||c||c||c||c||c|}
\hline \hline Game & ADS? & Avg. NC & Imp. & Std. Dev. & P-Value & Effect \\
\hline Virus Game & No & 264,200 & - & 32,400 & $3.0 \times 10^{-5}$ & 0.84 \\
Virus Game & Yes & 237,000 & $10 \%$ & 29,100 & $3.0 \times 10^{-5}$ & 0.84 \\
\hline Focus & No & $6,859,100$ & - & 685,300 & $1.9 \times 10^{-3}$ & 0.61 \\
Focus & Yes & $6,443,900$ & $6 \%$ & 805,300 & $1.9 \times 10^{-3}$ & 0.61 \\
\hline Chinese Checkers & No & $1,380,900$ & - & 379,400 & $5.4 \times 10^{-3}$ & 0.50 \\
Chinese Checkers & Yes & $1,191,700$ & $14 \%$ & 336,600 & $5.4 \times 10^{-3}$ & 0.50 \\
\hline
\end{tabular}

Consider Table 3.4, where we present our results for five players. The improvements here are generally larger still, with a reduction of $9 \%$ for the Virus Game, $10 \%$ for Chinese Checkers when no opponent is across from the player, and a particularly large $20 \%$ when no opponent is adjacent to the player (from 2, 501, 600 to 2, 095, 100). The superior results in the second case fit our expectations. Our analysis confirms that, in fact, both Chinese Checkers arrangements produced statistically significant results, although the second has a slightly larger Effect Size. Again, though, the actual reduction is larger in the second case, and is in fact our largest improvement yet.

Finally, Table 3.5 holds our results for the six player game. Here we see the results from the previous experiments in Chinese Checkers repeated. The Virus Game sees an improvement of $7 \%$ reduction in the tree size, which is in line with previous results. Again, these values show a medium to high Effect Size, and that the results are 
Table 3.4: Results by Game Model - Five Players

\begin{tabular}{|c||c||c||c||c||c||c|}
\hline \hline Game & ADS? & Avg. NC & Imp. & Std. Dev. & P-Value & Effect \\
\hline Virus Game & No & 265,600 & - & 29,800 & $9.0 \times 10^{-5}$ & 0.79 \\
Virus Game & Yes & 242,000 & $9 \%$ & 31,100 & $9.0 \times 10^{-5}$ & 0.79 \\
\hline Chinese Checkers (1) & No & $2,419,400$ & - & 589,100 & $9.3 \times 10^{-3}$ & 0.38 \\
Chinese Checkers (1) & Yes & $2,191,600$ & $10 \%$ & 872,100 & $9.3 \times 10^{-3}$ & 0.38 \\
\hline Chinese Checkers (2) & No & $2,501,600$ & - & 895,700 & 0.011 & 0.45 \\
Chinese Checkers (2) & Yes & $2,095,100$ & $20 \%$ & 596,000 & 0.011 & 0.45 \\
\hline
\end{tabular}

statistically significant within $95 \%$ certainty.

Table 3.5: Results by Game Model - Six Players

\begin{tabular}{|c||c||c||c||c||c||c|}
\hline \hline Game & ADS? & Avg. NC & Imp. & Std. Dev. & P-Value & Effect \\
\hline Virus Game & No & 284,000 & - & 33,500 & $2.3 \times 10^{-3}$ & 0.79 \\
Virus Game & Yes & 264,400 & $7 \%$ & 33,300 & $2.3 \times 10^{-3}$ & 0.79 \\
\hline Chinese Checkers & No & $3,485,400$ & - & 850,300 & 0.014 & 0.49 \\
Chinese Checkers & Yes & $3,070,400$ & $12 \%$ & 836,600 & 0.014 & 0.49 \\
\hline
\end{tabular}

\subsection{Variation on the Starting State of the Game}

Our previous results clearly demonstrate that the Threat-ADS heuristic is able to obtain a noticable improvement in the games that we have studied, regardless of the number of players (barring the well-understood and expected exception in the case of three-player Chinese Checkers). However, although we ran the game over several turns, these results only deal with the starting state of the game and a small number of moves into the future. In our present study, we are interested in learning how the Threat-ADS heuristic performs in a game that has advanced closer to a mid-game state $^{4}$.

\footnotetext{
${ }^{4}$ We are grateful to the committee members of the Thesis Proposal who suggested this analysis.
} 
To this end, we have run another set of experiments, analogous to those presented in the previous section, where we have advanced the game to a position some "distance" from the starting state. We have accomplished this by allowing the game to run for a set number of turns before we begin to take measurements, and then proceeded to measure the Node Count for the same number of turns as described above. Rather than running the game using random players to this midgame state, we have, instead, made all players intelligent, so as to obtain a more realistic board position. Specifically, the game was permitted to run having all opponents make use of a 2-ply BRS, until the specified midgame state. Afterwards, we reverted to the perspective player running the 4-ply BRS, and the others being random, as with previous experiments.

The number of turns we permitted the game to run for before taking measurements varied between the games. After some observation, we chose 15 turns for the Virus Game, 10 for Chinese Checkers, and 5 for Focus, as these numbers brought us to a more natural mid-game state, with a high level of variation in reasonable board positions. We then recorded the Node Count over the same number of turns as in the previous experiments. As before, we make use of the same statistical tests. Specifically, we again employ the Mann-Whitney test to determine significance, and provide the Effect Size measure, as we do not make the assumption of normalcy.

Our results are presented below.

\subsubsection{Results}

Table 3.6 displays our results for the three player case. We see the results are comparable to those found at the start of the game, with the Virus Game and Focus seeing $9 \%$ and $13 \%$ improvements in tree sizes, respectively, where, for example, in Focus, the NC was reduced from 15,159, 400 to 13,183,000. Again, Chinese Checkers did not provide any improvement. The possible reasons for this will be discussed later in this chapter. 
Table 3.6: Results by Game Model - Three Players - Midgame Start

\begin{tabular}{|c||c||c||c||c||c||c|}
\hline \hline Game & ADS? & Avg. NC & Imp. & Std. Dev. & P-Value & Effect \\
\hline Virus Game & No & 303,100 & - & 36,900 & $<1.0 \times 10^{-5}$ & 0.77 \\
Virus Game & Yes & 274,700 & $9 \%$ & 29,500 & $<1.0 \times 10^{-5}$ & 0.77 \\
\hline Focus & No & $15,159,400$ & - & $3,368,300$ & $1.8 \times 10^{-3}$ & 0.59 \\
Focus & Yes & $13,183,000$ & $13 \%$ & $2,553,000$ & $1.8 \times 10^{-3}$ & 0.59 \\
\hline Chinese Checkers & No & $1,251,000$ & - & 400,000 & 0.42 & 0.02 \\
Chinese Checkers & Yes & $1,262,700$ & $-1 \%$ & 377,600 & 0.42 & 0.02 \\
\hline
\end{tabular}

Table 3.7 presents our results for when there are four players in the game. The reductions appear to be in line with the previous results, showing $7 \%, 10 \%$, and $13 \%$ reductions for the Virus Game, Focus, and Chinese Checkers respectively. However, it was observed that five turns into a four-player Focus game was causing significant deviation in the number of captured pieces for each player. Therefore, we opted to run this experiment with a three turn setup, as well, instead of five, which generated a particularly large $19 \%$ reduction in tree size, from 16,767, 600 to $13,592,300$. On the other hand, five-turn Focus does show a visible reduction in tree size, and as a result it falls just outside the $95 \%$ certainty level, and the corresponding Effect Size is very low. Our three turn experiment, however, achieves a 95\% certainty level.

Table 3.7: Results by Game Model - Four Players - Midgame Start

\begin{tabular}{|c||c||c||c||c||c||c|}
\hline \hline Game & ADS? & Avg. NC & Imp. & Std. Dev. & P-Value & Effect \\
\hline Virus Game & No & 307,100 & - & 38,400 & $6.6 \times 10^{-4}$ & 0.53 \\
Virus Game & Yes & 286,700 & $7 \%$ & 27,200 & $6.6 \times 10^{-4}$ & 0.53 \\
\hline Focus & No & $14,458,000$ & - & $6,546,500$ & 0.051 & 0.21 \\
Focus & Yes & $13,050,400$ & $10 \%$ & $9,806,700$ & 0.051 & 0.21 \\
\hline Focus (3-turn) & No & $16,767,600$ & - & $7,160,900$ & 0.01 & 0.44 \\
Focus (3-turn) & Yes & $13,592,300$ & $19 \%$ & $5,234,600$ & 0.01 & 0.44 \\
\hline Chinese Checkers & No & $3,438,100$ & - & 904,800 & 0.01 & 0.46 \\
Chinese Checkers & Yes & $3,024,300$ & $12 \%$ & 816,400 & 0.01 & 0.46 \\
\hline
\end{tabular}


Consider Table 3.8, where we present our results for the five player case. The reductions, and their statistical qualities, are approximately what we have come to expect, i.e., a $9 \%$ reduction for the Virus Game, and a $10 \%$ and $9 \%$ reduction for the two Chinese Checkers cases, respectively (where the perspective player has no opponent across from him, and when he has no opponent adjacent to him). In the case of Chinese Checkers, an increased variance leads to a lower Effect Size, and, in the second case, when the player has no opponent adjacent to them, our results fall marginally outside being statistical significant. We will undertake a discussion of this case later in this chapter.

Table 3.8: Results by Game Model - Five Players - Midgame Start

\begin{tabular}{|c||c||c||c||c||c||c|}
\hline \hline Game & ADS? & Avg. NC & Imp. & Std. Dev. & P-Value & Effect \\
\hline Virus Game & No & 305,500 & - & 35,000 & $2.5 \times 10^{-4}$ & 0.82 \\
Virus Game & Yes & 276,700 & $9 \%$ & 40,000 & $2.5 \times 10^{-4}$ & 0.82 \\
\hline Chinese Checkers (1) & No & $6,121,800$ & - & $1,810,000$ & 0.017 & 0.36 \\
Chinese Checkers (1) & Yes & $5,469,900$ & $10 \%$ & $1,570,000$ & 0.017 & 0.36 \\
\hline Chinese Checkers (2) & No & $7,171,700$ & - & $2,463,800$ & 0.099 & 0.27 \\
Chinese Checkers (2) & Yes & $6,491,700$ & $9 \%$ & $2,035,800$ & 0.099 & 0.27 \\
\hline
\end{tabular}

Lastly, Table 3.9 holds our results for the six player game. Here we see a reduction of $10 \%$ for the Virus Game, from 299, 300 to 268,000, and a 6\% reduction in the case of Chinese Checkers. We see another incidence of a very small $P$-value for the Virus Game here, although the results for Chinese Checkers were not statistically significant.

Table 3.9: Results by Game Model - Six Players - Midgame Start

\begin{tabular}{|c||c||c||c||c||c||c|}
\hline \hline Game & ADS? & Avg. NC & Imp. & Std. Dev. & P-Value & Effect \\
\hline Virus Game & No & 299,300 & - & 35,100 & $<1.0 \times 10^{-5}$ & 0.89 \\
Virus Game & Yes & 268,000 & $10 \%$ & 32,700 & $<1.0 \times 10^{-5}$ & 0.89 \\
\hline Chinese Checkers & No & $8,167,800$ & - & $2,564,200$ & 0.18 & 0.30 \\
Chinese Checkers & Yes & $7,676,900$ & $6 \%$ & $2,282,700$ & 0.18 & 0.30 \\
\hline
\end{tabular}




\subsection{Evaluation of Results}

The results described above are in line with our hypothesis. They demonstrate that a clear improvement, in terms of tree pruning, can be obtained by applying the ThreatADS heuristic to the BRS. This implies that better move ordering is, indeed, achieved through the use of the Threat-ADS heuristic, and confirms that the ADS is able to successfully identify the most powerful opposing player, and place his moves towards the head of the list. This also confirms that the Threat-ADS performs in accordance with the arguments laid out earlier in this chapter.

The first conclusion we can draw is that the Threat-ADS heuristic can produce statistically significant reductions in tree sizes in the context of three multi-player board games, the Virus Game, Chinese Checkers, and Focus. The degree of the reduction varies between the three games, ranging between $5 \%$ and $20 \%$, and generally centered around a 10\% reduction in tree size. However, generally speaking, Chinese Checkers saw the largest relative reduction in terms of its Node Count, compared to the Virus Game and Focus, except for those situations where the particular configuration of the game limited the ability of any specific opponent to minimize the perspective player, such as the three-player, initial board position case. This is in contrast to results we originally presented in [37], where the reduction for Chinese Checkers, and the statistical certainty of the results, was the smallest of all the games. Given that the same software system was used in gathering our results in this chapter, we suspect that the results in [37] may have been somewhat anomalous, as we have performed a much larger number of experiments involving Chinese Checkers in this work.

Our second conclusion is that the Threat-ADS has the capability to produce valid results when presented with a varied number of opponents. When considering the starting board position, a statistically significant benefit was detected in all the cases examined, except for the three player Chinese Checkers game. As alluded to earlier, in fact, it fits expectations that the Threat-ADS would fail to produce good results in that specific case. The reasoning behind this is that, as no player is adjacent to 
another at the start of the game, there is no capacity for either opponent to minimize the perspective player, and thus there is no meaningful relative threat between the two opponents. In all the other cases examined, there is a clear improvement by invoking the Threat-ADS, and this occurs with a strong statistical certainty.

We can also conclude that, in the majority of cases examined, the Threat-ADS is also capable of generating meaningful reductions in tree size when utilized within the context of a mid-game state. This is a valuable observation, as the opening moves of the game are not necessarily the most interesting, and mid-game states naturally demonstrate a much higher degree of variability than the opening position. As with the starting position, the three player Chinese Checkers game did not generate statistically significant improvements, nor did it yield any real change in its performance at all. It is somewhat more surprising that in the mid-game in the midgame scenario, when the players are capable of minimizing each other in the three player case, no improvement is noticed. A possible explanation could be that neither of the two opponents tends to threaten the perspective player more than his peer, as the board is symmetrical in this case, or that there are not enough pieces on the board in the three player game to block a piece's forward movement effectively.

We also note that we did not obtain statistically significant results in the midgame experiments for four player Focus, when run from the fifth turn, from five player Chinese Checkers, when no opponent is adjacent to the perspective player, and for six player Chinese Checkers. We observe, however, that in all these cases the average Node Counts were, nevertheless, lower when the Threat-ADS was applied. Furthermore particularly high variances were observed in the mid-game experiments. This is to be expected, as we are considering a broad range of possible starting positions for our measurements in these experiments. We consequently conjecture that the high variance may be impeding the appearance of statistically significant results in these cases, even though the reduction in the $\mathrm{NC}$ was consistent. In any case, to avoid presenting misleading results, we maintained the sample size to be consistent between our experiments. 
The Effect Size metric for our experiments ranges between 0.36 and 0.89, when a statistically significant benefit from the Threat-ADS was observed. In interpreting the Effect Size, the commonly discussed values are 0.2 for a small, but noticeable, effect, 0.5 for a medium effect, and 0.8 for a large, very noticable effect $[11,69]$. A well-known weakness of statistical significance tests is that they are dependent both on the size of the effect being tested, and the sample size itself [11]. Therefore, these observed Effect Sizes allow us to conclude that, in general, a medium to large effect is being observed from the application of the Threat-ADS heuristic, and that our statistically significant results are not simply due to oversampling. Thus, we believe that we can confidently confirm that the Threat-ADS heuristic yields a noticeable impact on the tree size produced by the BRS.

The average running time of the experiments was highly variable between the three games used. Running the set of fifty experiments in the context of the Virus Game could be done in roughly a minute, ten to forty minutes in the case of Chinese Checkers (roughly evenly distributed across that range, and increasing with the number of players), and a substantially longer three to five hours in the case of Focus, increasing with both the number of players, and it being even longer when beginning at a midgame board state. Our observations on runtime also support Schaeffer's statements about the Node Count index being correlated to the runtime, with the experiments using the Threat-ADS heuristic taking roughly $10 \%$ less time, on average, than those not employing it.

The reader will observe that, concerning the general behaviour of the ThreatADS's performance, a benefit was obtained in the vast majority of the cases examined and in all three of our chosen game models. As these games differ noticeably in how they are played and the player's objectives, we believe it is reasonable to hypothesize that the Threat-ADS heuristic will also produce useful results in other multi-player board games as well, where players can minimize each other.

These initial results for the Threat-ADS heuristic are particularly promising, as they represent a relatively intuitive application of ADSs to multi-player game playing, 
which is yet able to achieve noticeable results in a number of different cases. As such, since an intuitive and inexpensive heuristic, with a relatively simple application of techniques from the field of ADSs, has produced admissible results, we believe that these results provide a powerful basis for further investigation into incorporating techniques from ADSs into game playing strategies.

\subsection{Chapter Conclusions}

In this chapter, we have presented a heuristic for the BRS, which we have called Threat-ADS, which uses an ADS to achieve move ordering for improved node pruning. This work represents the first reported instance of the merging of ADSs and multiplayer game playing in the literature, as well as the first use of relative opponent threat levels in improving move ordering. We have tested this lightweight heuristic in the context of three multi-player board games, the Virus Game, Focus, and Chinese Checkers, and found that it produces statistically significant results.

Even though the results from this chapter clearly show the efficacy of the ThreatADS heuristic, a number of questions remain open. First of all, in this chapter we exclusively used the "Move-to-Front" update mechanism, although a number of adaptive lists were discussed in Chapter 2.7. Different update mechanisms will lead to a different ranking of the opponents, that could influence, for better or worse, the performance of Threat-ADS. We also note that we have tested the Threat-ADS heuristic only at a 4-ply depth, although it is known that other move ordering heuristics, such as the History heuristic, can lead to a decayed performance in deeper trees [57]. At this juncture, the performance of the Threat-ADS heuristic in deeper trees is unknown.

In the next chapter, we will investigate the above unanswered questions with regards to the Threat-ADS heuristic. Our goal will be to glean a more comprehensive knowledge of its performance, and furthermore, to provide guidance to future attempts to merge ADSs and multi-player game playing. 


\section{Chapter 4}

\section{Improvements to the Threat-ADS Heuristic}

\subsection{Chapter Overview}

In the previous chapter, we introduced the concept of improving multi-player game playing, through techniques derived from the field of ADSs. We proposed and explored the phenomena associated with relative opponent threat levels, and described how a data structure, using established update mechanisms derived from ADSs, can both record and learn these threat levels. Within the context of the BRS, we showed how such a data structure can be naturally updated and used, leading to the specification and design of the Threat-ADS heuristic. Our experiments showed that the ThreatADS heuristic performed well, under the game models discussed in detail in Appendix $A$, in an ensemble of cases that involved varying the number of opponents and the starting point of the game. These results, as discussed previously, show the potential benefits of even a simple application of ADSs. 
In this chapter, we first review a few open questions from the work presented in the previous chapter, and construct a second ensemble of experiments designed to address these new avenues of investigation ${ }^{1}$.

\subsection{Open Questions}

A number of questions can be considered "open" after the work presented in Chapter 3. Of these avenues, the obvious question for deeper investigation is that of invoking other ADS update mechanisms. The results of Chapter 3 clearly demonstrate that the Threat-ADS using the Move-to-Front update mechanism produces admissible results. But, at this current juncture, we do not know if other ADSs are capable of achieving an analogous goal. Furthermore, it could well be the case that other ADSs could outperform the Move-to-Front, leading to a further reduction in the index of interest, i.e., NC. Inquiries related to involving other ADSs are of particular interest, inasmuch as if an alternate ADS is used, the ranking that the Threat-ADS arrives upon could be substantially different, and thus the derived move orderings and prunings in the search tree will be distinct. Finally, given that a specific game's relative opponent threat based on the board position is necessarily dependent upon the individual game model, it may be the case that two ADSs could perform the same under one game model, and differently under another, or that a superior ADS may be inferior when the game model is changed. Indeed, none of these issues have a clear answer, and this warrants investigation.

The second avenue of investigation is related to the depth of the search tree. As discussed earlier in this work, for a variety of reasons, certain heuristics that can perform very well in shallow search trees may be unable to replicate that performance

\footnotetext{
$[43]$

${ }^{1}$ The results in this chapter were originally presented in [38] and [40], and in the journal paper
} 
in deeper trees. While we do not currently have the resources to test the ThreatADS heuristic in very deep trees (in part because we are not making use of domainspecific techniques, but are, rather, interested in investigating techniques that can be applied to a variety of game models), it is worthwhile to examine if its performance is hampered, or even improved, in deeper search trees.

The above points motivate the study undertaken in this chapter.

\subsection{Threat-ADS' Behaviour Using Different ADSs}

To showcase how the move ordering derived from the Threat-ADS can vary, consider Figure 4.1, where the Threat-ADS adjusts its relative opponent levels based on the Move-to-Front update mechanism.

We now contrast the above with Figure 4.2, where the Threat-ADS heuristic operates by working in conjunction with the Transposition update mechanism. Notice that the same move is found to be the most threatening. However, in this case, the resultant data structure, and therefore its perception of the relative opponent threats, is different. Consequently, the next time it is invoked for the purposes of move ordering, the order in which the opponents' moves are explored, is different, which, of course, could lead to a significantly different tree pruning.

The fact that different opponent rankings can be achieved by merely changing the ADS used is certainly interesting. However, this observation would have limited applicability if it were simply an arbitrary decision. One should rather observe that the different ADS update mechanisms could have different motivations in their usage, as well. For example, the Move-to-Front rule is particularly sensitive, since it makes broad changes to the structure based on its input. By contrast, the Transposition rule is less sensitive, since it leads to only marginal changes in the rankings of the players. The Move-Ahead- $k$ and $\operatorname{POS}(k)$ update mechanisms, discussed in Section 2.7, attempt to strike a compromise between these two extreme approaches. Thus, 


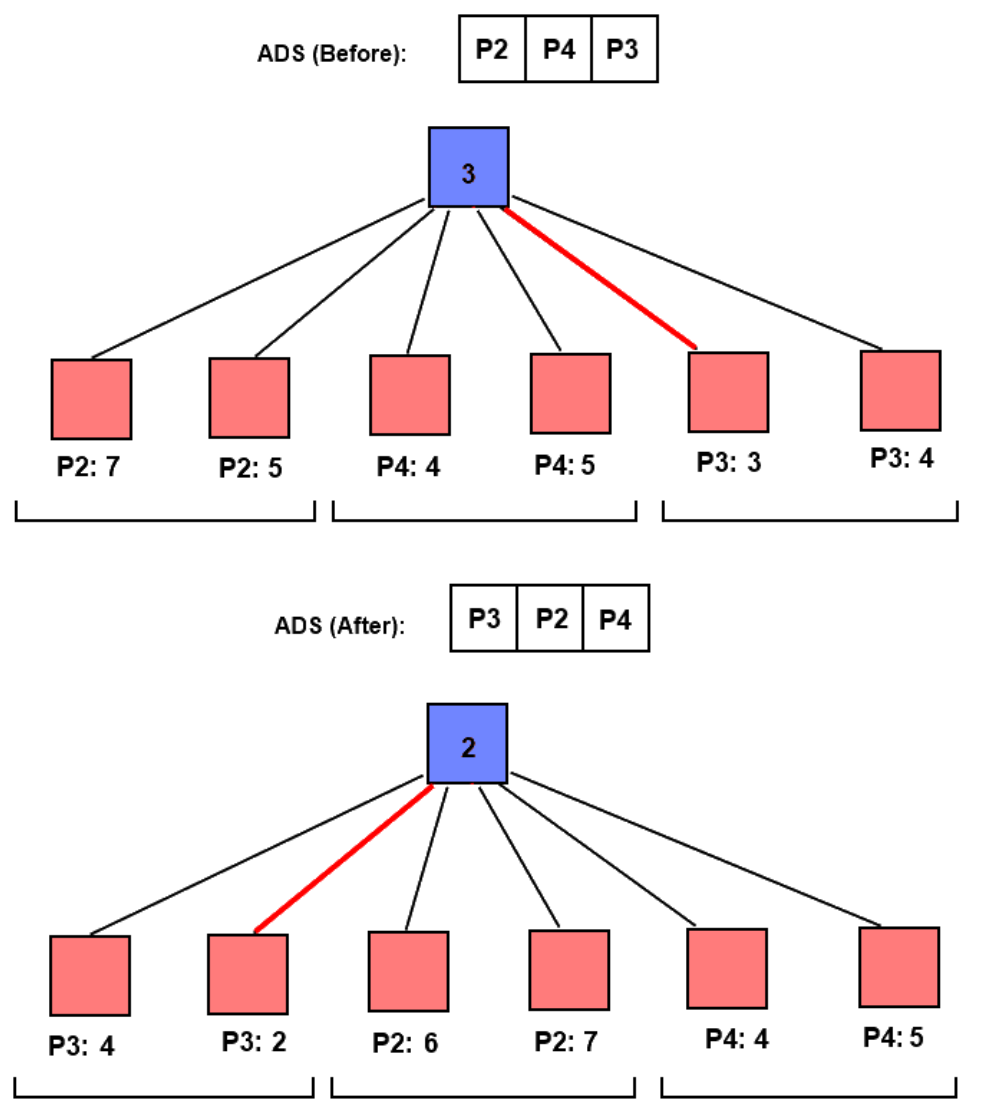

Figure 4.1: A demonstration of the Threat-ADS heuristic updating using a Move-toFront ADS, obtained by moving player P3 to the front of the list.

the decision of which ADS is to be used could be made based on the ADS' qualities, and in relation to the nature of the game model, and it could thus be an informed decision. 


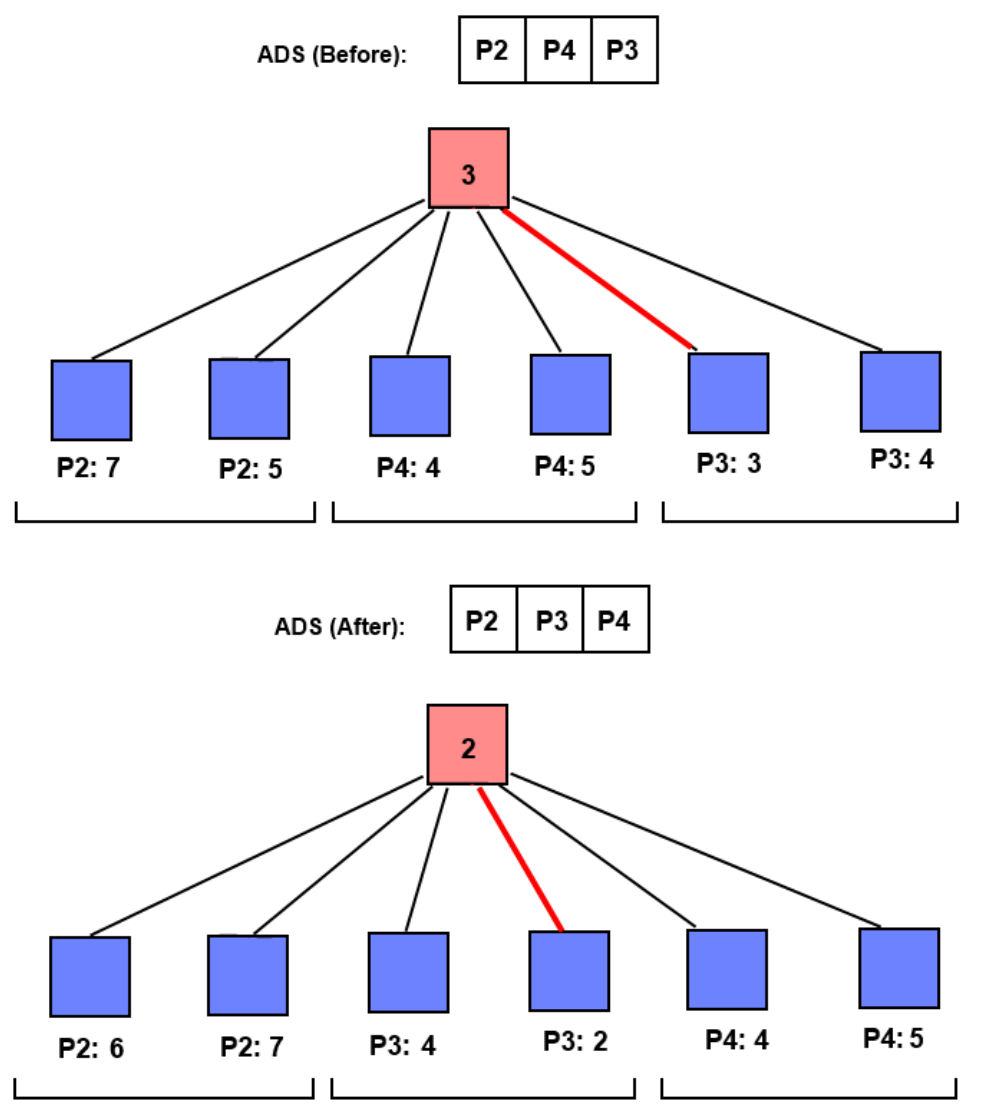

Figure 4.2: A demonstration of the Threat-ADS heuristic updating using a Transposition ADS, obtained by moving player P3 one step closer to the front of the list.

\subsection{Ergodic versus Absorbing ADSs}

While a variety of different update mechanisms can lead to distinct relative opponent threat rankings, and their consequent resultant move orderings, an important distinction exists between ergodic and absorbing ADSs, as explained in Section 2.7. To summarize, an absorbing update mechanism, such as the Stochastic Move-to-Rear rule, eventually converges to a final structure, from which it will not deviate, regardless of future queries. This quality could perhaps be leveraged by the Threat-ADS in 
a situation where, while relative opponent threats will vary throughout the game, one opponent will consistently pose a much greater threat to the perspective player for the majority of the game. An absorbing update mechanism may be able to quickly converge to an arrangement that prioritizes that player, undisturbed by random chance. We showcase an example of a situation where this could be valuable in Figure 4.3, where the same players are likely to vie for control of a particular corner of the board.

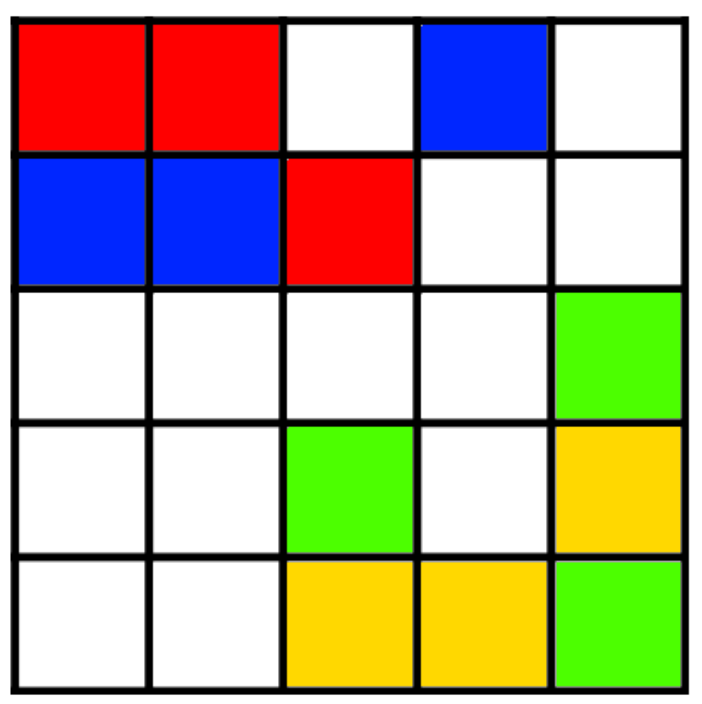

Figure 4.3: An example of two players threatening each other in the Virus Game.

Therefore, although we suspect that changing board positions could cause absorbing update mechanisms to perform worse, we consider it worthwhile to investigate the performance of an absorbing update mechanism in the Threat-ADS heuristic, so as to investigate if its effect can be leveraged in any of our game models. 


\subsection{Investigating the Threat-ADS' Performance at Different Ply Levels}

It is known that certain move ordering heuristics, particularly the highly-regarded History heuristic, have a reduced impact in trees with a larger ply, as the number of turns that the algorithm is permitted to look ahead increases. This can eventually lead to the heuristic being essentially random noise, as it has been reported in the case of the History heuristic [57]. It is therefore, pertinent to understand how the Threat-ADS heuristic performs in deeper trees. Specifically, we are interested in determining if its performance is reduced in larger trees, which would indicate that it does, indeed, decay in very deep trees. Using the method used in the previous chapter, such a phenomenon would be represented by a reduction in the change in the NC measure.

Intuitively, we can hypothesize how and why the Threat-ADS heuristic may be susceptible to decay in deeper trees. This is because it maintains a single ADS, which is used at all levels of the tree, which are explored in a depth-first manner. Therefore, it is easy to envision how the ADS may adapt to relative opponent threat levels several turns into the future in a deeper tree, and then have that information applied earlier - when it is not really applicable. For example, consider Figure 4.4, where we see how, after several turns, the relative opponent threat levels have changed, and a new opponent is the most threatening one to the perspective player. It could be that this effect will cause a reduction in the performance of the Threat-ADS heuristic in a deeper tree.

However, given how quickly the ergodic update mechanisms, in particular, can adapt to a change in the threat levels, it is possible that this effect will not appear. This clearly begs investigation. On the other hand, it may also be the case that different ADSs will be superior in deeper trees, as opposed to shallow ones. 


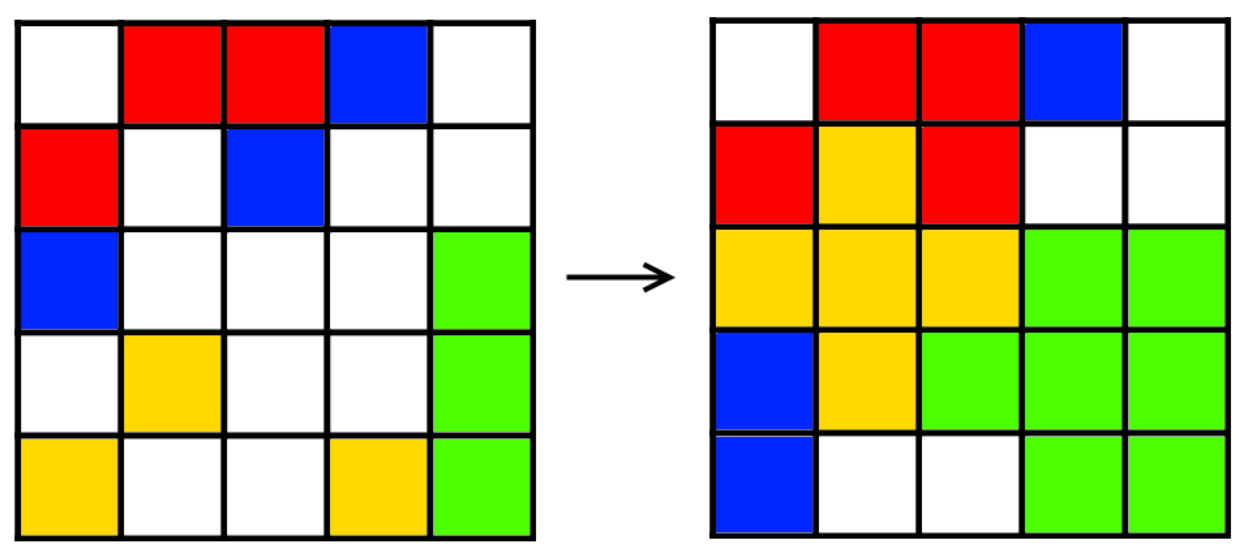

Figure 4.4: An example of how the relative threat levels of opponents can change with time.

\subsection{Experimental Model}

Our experiments in this chapter use the same software system and configurations as in the preceding chapter, as we wish to be able to compare this chapter's work to our previous results. We are interested in determining the improvement in tree pruning from the use of the Threat-ADS heuristic, by making use of a variety of list-based ADS update mechanisms. We are furthermore interested in testing the Threat-ADS heuristic in deeper trees with all of the ADS update mechanisms that we explore. The list-based ADS update mechanisms that we will examine are the Move-to-Front, Transposition, Move-Ahead- $k, \operatorname{POS}(k)$ and the absorbing Stochastic Move-to-Rear.

Each individual experiment uses the same configurations described in the previous chapter. We measure the NC over several turns, which we then aggregate and average over fifty trials. The number of turns that we do this for depends on the game in question, with the same values used in Section 3.6 (ten for the Virus Game, five for Chinese Checkers, and three for Focus). In the case of experiments beginning from a midgame state, we generate the starting position in the same way as we did 
in Chapter 3. The exception to this is for the Virus Game, where we increase the number of players to five, because by doing this the Move-Ahead- $k$ and $\operatorname{POS}(k)$ will be rendered distinct, and will not reduce to the Move-to-Front and Transposition respectively (which will be the case if the list of opponents is of size 3). For the same reason, we have set the value of $k$ to be two. Finally, we have set the learning parameter of the Stochastic Move-to-Rear rule to be 0.99 , to insure that it does not rapidly converge to a final structure.

The experiments we presented in the previous chapter covered a range of factors, including the game model, the number of players, and whether the recording of the measurements began at the starting position of the game or from a midgame state. The work in this chapter adds the update mechanism and the ply depth as variable factors, which were considered static in the context of the previous chapter's work. While a possible option would be to exhaustively repeat all our former experiments with each alternative update mechanism and at deeper ply depths, such an approach is impractical, as each new factor examined would have a multiplicative effect on the number of experiments required. This would take a substantial amount of time, given the runtimes discussed in Section 3.10, and would lead to a large number of likely redundant results. Based on our observations from the previous chapter's work, we have thus decided upon reporting here only a subset of all possible experiments.

As the number of players was not generally found to have a substantial impact on the performance of Threat-ADS, we have chosen to repeat those experiments described in Section 3.7 for each available update mechanism, with the number of players in the Virus Game being increased to five for the reasons discussed above. In addition to this, we also re-mention the single scenario where the number of players did make a major difference, which occurred when comparing six-player Chinese Checkers to four-player Chinese Checkers. We have thus included both of these settings. However, we have omitted the three-player Chinese Checkers scenario, given its failure to produce useful results in that case, as explained in Section 3.10. Also, in the interest of completeness, we have included the special case for midgame four-player Focus, where the game was run for three (or five) turns before any measurements were recorded. 
When considering deeper ply depths, we have tested the Virus Game at a 6ply search depth, with all the update mechanisms mentioned. Given that Chinese Checkers and Focus would take between ten to forty minutes, and three to five hours, respectively, to run to completion at a 4-ply depth, it is not feasible, at this juncture, to test these games at a 6-ply depth. As the average branching factor for both these games is over twenty for both the perspective player and the opponent, the expected time to completion for fifty trials could be well over a week for both of the games, under the current hardware limitations.

\subsection{Initial Board Position Experiments}

We now present our results for those experiments starting from the initial board state, as well as their statistical analysis. These results will be followed by those starting from a midgame board position. As before, we employ the Mann-Whitney test to determine statistical significance, and provide the Effect Size metric to demonstrate the degree of the Threat-ADS' impact.

Table 4.1 shows our results for the Virus Game at a 4-ply search depth. The values when we invoked no ADS (i.e., when there was no invocation of the ThreatADS heuristic) and when it involved the Move-to-Front are from the previous chapter. From this table, we see that invoking any other ergodic ADS produces a similar reduction in tree size, and all these reductions are statistically significant according to the Mann-Whitney test. However, we do observe a slightly smaller improvement if the Transposition rule is used, and a correspondingly smaller Effect Size. As was expected, no real improvement was generated from the absorbing Stochastic Moveto-Rear rule. The best scenario was for the $\operatorname{POS}(K)$ rule, where the improvement is $11 \%$, from 265,600 to 237,000 .

We note that the difference between the $\operatorname{POS}(k)$ and Transposition rules is itself statistically significant, with a $P$-Value of 0.02 , indicating that we may reject the null hypothesis that this change is due to sampling error. 
Table 4.1: Results for the 4-ply Virus Game when the measurement started from an initial game position.

\begin{tabular}{|c||c||c||c||c||c|}
\hline \hline Update Mech. & Avg. NC & Imp. & Std. Dev. & P-Value & Effect \\
\hline None & 265,600 & - & 29,800 & - & - \\
\hline Move-to-Front & 242,000 & $9 \%$ & 31,100 & $1.2 \times 10^{-4}$ & 0.79 \\
Transposition & 250,000 & $6 \%$ & 30,300 & $6.7 \times 10^{-3}$ & 0.53 \\
Move-Ahead- $k$ & 241,600 & $10 \%$ & 39,500 & $1.1 \times 10^{-4}$ & 0.80 \\
POS $(k)$ & 237,000 & $11 \%$ & 29,100 & $<1.0 \times 10^{-5}$ & 0.96 \\
Stochastic MTR & 264,300 & $1 \%$ & 42,800 & 0.41 & 0.04 \\
\hline
\end{tabular}

Table 4.2 contains the results obtained for the 6-ply Virus Game. Here, we can observe a similar consistency between the ergodic data structures, although the improvement is noticeably larger. Again, the Stochastic Move-to-Rear rule failed to produce any real benefit. The best result here was for the Move-Ahead- $k$ update mechanism, which yielded a 15\% improvement, from 11,758, 600 to 10,012,600. We see, in this case, correspondingly very large Effect Sizes, matching the improvement in tree pruning. All ergodic update mechanisms produced a very small $P$-Value, and again, the Stochastic Move-to-Rear difference was not statistically significant.

Table 4.2: Results for the 6-ply Virus Game when the measurement started from an initial game position.

\begin{tabular}{|c||c||c||c||c||c|}
\hline \hline Update Mech. & Avg. NC & Imp. & Std. Dev. & P-Value & Effect \\
\hline None & $11,758,600$ & - & $1,433,000$ & - & - \\
\hline Move-to-Front & $10,027,800$ & $15 \%$ & $1,530,100$ & $<1.0 \times 10^{-5}$ & 1.21 \\
Transposition & $10,249,800$ & $13 \%$ & $1,615,400$ & $<1.0 \times 10^{-5}$ & 1.05 \\
Move-Ahead- $k$ & $10,012,600$ & $15 \%$ & $2,007,900$ & $<1.0 \times 10^{-5}$ & 1.22 \\
POS $(k)$ & $10,201,000$ & $13 \%$ & $1,263,300$ & $<1.0 \times 10^{-5}$ & 1.09 \\
Stochastic MTR & $11,863,100$ & $-1 \%$ & $2,298,700$ & 0.18 & -0.07 \\
\hline
\end{tabular}


We display our results for the four-player Chinese Checkers case in Table 4.3. Here, as there are only three opponents, the Move-Ahead- $k$ and $\operatorname{POS}(k)$ operate identically to the Move-to-Front and Transposition, respectively, and so the values are duplicated, and marked with a "*". They are included here to maintain consistency and readability between the tables. From the results, we observe a slightly better performance from the Move-to-Front update mechanism, compared to the Transposition rule. Again, all ergodic structures generated an improvement, while the absorbing structure did not. The best strategy was the Move-to-Front, generating a $14 \%$ reduction in tree size, from 1,380, 900 to 1,191,700. Although the Transposition/POS $(k)$ produced a smaller change and Effect Size than the Move-to-Front/Move-Ahead- $k$, this difference is not significant, with a $P$-Value of 0.35 .

Table 4.3: Results for the 4-player Chinese Checkers scenario when the measurement started from an initial game position.

\begin{tabular}{|c||c||c||c||c||c|}
\hline \hline Update Mech. & Avg. NC & Imp. & Std. Dev. & P-Value & Effect \\
\hline None & $1,380,900$ & - & 379,400 & - & - \\
\hline Move-to-Front & $1,191,700$ & $14 \%$ & 336,600 & $5.4 \times 10^{-3}$ & 0.50 \\
Transposition & $1,248,100$ & $10 \%$ & 398,300 & 0.02 & 0.35 \\
Move-Ahead- $k^{*}$ & $1,191,700$ & $14 \%$ & 336,600 & $5.3 \times 10^{-3}$ & 0.50 \\
POS $(k)^{*}$ & $1,248,100$ & $10 \%$ & 398,300 & 0.02 & 0.35 \\
Stochastic MTR & $1,391,000$ & $-0.5 \%$ & 413,800 & 0.49 & 0.03 \\
\hline
\end{tabular}

Consider Table 4.4, where we present our results for six-player Chinese Checkers. Again, the ergodic structures all generate an improvement, and the Stochastic Moveto-Rear does not. However, this time the improvement from using the Move-to-Front was highest at a $12 \%$ improvement, and the Move-Ahead- $k$ is noticeably superior to the Transposition rule and $\operatorname{POS}(k)$, a phenomenon which will be discussed later in this chapter. Furthermore, we observe that while the Move-to-Front and MoveAhead- $k$ produced statistically significant improvements in their tree pruning effects, the Transposition and $\operatorname{POS}(k)$ did not. 
Table 4.4: Results for the 6-player Chinese Checkers scenario when the measurement started from an initial game position.

\begin{tabular}{|c||c||c||c||c||c|}
\hline \hline Update Mech. & Avg. NC & Imp. & Std. Dev. & P-Value & Effect \\
\hline None & $3,485,400$ & - & 850,300 & - & - \\
\hline Move-to-Front & $3,070,400$ & $12 \%$ & 836,600 & 0.01 & 0.49 \\
Transposition & $3,267,500$ & $7 \%$ & 924,700 & 0.07 & 0.26 \\
Move-Ahead- $k^{*}$ & $3,149,500$ & $10 \%$ & 888,800 & 0.04 & 0.40 \\
POS $(k)^{*}$ & $3,260,000$ & $6 \%$ & 926,500 & 0.08 & 0.26 \\
Stochastic MTR & $3,462,700$ & $1 \%$ & 957,800 & 0.48 & 0.02 \\
\hline
\end{tabular}

Finally, Table 4.5 shows our results for Focus. Again, the ergodic structures all do well, and roughly the same, and the absorbing structure failed to achieve any noticeable benefit. As Focus is a four-player game, again we see identical values for the Move-Ahead- $k$ and Move-to-Front, as well as for the $\operatorname{POS}(k)$ and Transposition, whose results have been included in the interest of completeness. The best scheme was the Transposition rule, with a $7 \%$ reduction in tree size. As before, all improvements, save for the Stochastic Move-to-Rear, are statistically significant.

Table 4.5: Results for Focus when the measurement started from an initial game position.

\begin{tabular}{|c||c||c||c||c||c|}
\hline \hline Update Mech. & Avg. NC & Imp. & Std. Dev. & P-Value & Effect \\
\hline None & $6,859,100$ & - & 685,300 & - & - \\
\hline Move-to-Front & $6,443,900$ & $6 \%$ & 805,300 & $1.9 \times 10^{-3}$ & 0.61 \\
Transposition & $6,376,200$ & $7 \%$ & 572,000 & $2.2 \times 10^{-3}$ & 0.70 \\
Move-Ahead- $k^{*}$ & $6,443,900$ & $6 \%$ & 805,300 & $1.9 \times 10^{-3}$ & 0.61 \\
POS $(k)^{*}$ & $6,376,200$ & $7 \%$ & 572,000 & $2.2 \times 10^{-3}$ & 0.70 \\
Stochastic MTR & $6,927,400$ & $-1 \%$ & 826,400 & 0.29 & -0.10 \\
\hline
\end{tabular}




\subsection{Midgame Starting Position Experiments}

Having completed our experiments dealing with the initial board state, we will now present our results, and the corresponding analysis, for those experiments starting from a midgame board position.

Table 4.6 shows our results for the 4-ply Virus Game. As before, all ergodic update mechanisms generate a statistically significant improvement, and the Stochastic Move-to-Rear does not. Our best results are with the Transposition rule, with a 10\% reduction in tree size, from 305,500 to 274,800 .

Table 4.6: Average Tree Size for the 4-ply Virus Game when the measurement started from a midgame game position.

\begin{tabular}{|c||c||c||c||c||c|}
\hline \hline Update Mech. & Avg. NC & Imp. & Std. Dev. & P-Value & Effect \\
\hline None & 305,500 & - & 35,000 & - & - \\
\hline Move-to-Front & 276,700 & $9 \%$ & 40,000 & $2.5 \times 10^{-4}$ & 0.82 \\
Transposition & 274,800 & $10 \%$ & 37,400 & $6.0 \times 10^{-5}$ & 0.87 \\
Move-Ahead- $k$ & 280,000 & $8 \%$ & 30,100 & $1.4 \times 10^{-4}$ & 0.72 \\
POS $(k)$ & 285,900 & $6 \%$ & 39,700 & $5.7 \times 10^{-3}$ & 0.56 \\
Stochastic MTR & 298,500 & $1.5 \%$ & 38,400 & 0.20 & 0.20 \\
\hline
\end{tabular}

Consider Table 4.7, which holds our results for the 6-ply Virus Game. Here, at the midgame state, we do not see the same increased performance when compared to the 4-ply case, although the results obtained match those observed earlier, and the performance does not decay. A commentary on the differing performances at different board positions is included in the next section. The best scheme again was a tie between the Transposition rule and the Move-Ahead- $k$, at $10 \%$.

Table 4.8 presents our results for four player Chinese Checkers. The results obtained again match the pattern we have observed all along, i.e., that all ergodic update mechanisms producing an improvement, although with the Transposition/POS $(k)$ having a smaller impact, and at a lower threshold of statistical significance, and the 
Table 4.7: Average Tree Size for the 6-ply Virus Game when the measurement started from a midgame game position.

\begin{tabular}{|c||c||c||c||c||c|}
\hline \hline Update Mech. & Avg. NC & Imp. & Std. Dev. & P-Value & Effect \\
\hline None & $12,468,600$ & - & $1,712,700$ & - & - \\
\hline Move-to-Front & $11,415,000$ & $9 \%$ & $1,315,100$ & $1.5 \times 10^{-3}$ & 0.65 \\
Transposition & $11,277,500$ & $10 \%$ & $1,515,100$ & $8.4 \times 10^{-4}$ & 0.70 \\
Move-Ahead- $k$ & $11,281,900$ & $10 \%$ & $1,419,900$ & $5.2 \times 10^{-4}$ & 0.69 \\
POS $(k)$ & $11,304,700$ & $10 \%$ & $1,712,700$ & $1.2 \times 10^{-3}$ & 0.68 \\
Stochastic MTR & $12,560,200$ & $-0.5 \%$ & $1,793,500$ & 0.36 & -0.05 \\
\hline \hline
\end{tabular}

Stochastic Move-to-Rear rule did not yield such a noticeable change. Observe that the Move-to-Front and Move-Ahead- $k$, as well as the Transposition rule and $\operatorname{POS}(k)$, are the same again due to this being a four-player game. The best results obtained in this case is for the Move-to-Front, with a $12 \%$ reduction in the tree size, from $3,438,100$ to $3,024,300$.

Table 4.8: Average Tree Size for the 4-player Chinese Checkers scenario when the measurement started from a midgame game position.

\begin{tabular}{|c||c||c||c||c||c|}
\hline \hline Update Mech. & Avg. NC & Imp. & Std. Dev. & P-Value & Effect \\
\hline None & $3,438,100$ & - & 904,800 & - & - \\
\hline Move-to-Front & $3,024,300$ & $12 \%$ & 816,400 & 0.01 & 0.46 \\
Transposition & $3,206,200$ & $7 \%$ & 798,400 & 0.12 & 0.26 \\
Move-Ahead- $k^{*}$ & $3,024,300 *$ & $13 \%$ & 816,400 & 0.01 & 0.46 \\
POS $(k)^{*}$ & $3,206,300 *$ & $7 \%$ & 798,400 & 0.12 & 0.26 \\
Stochastic MTR & $3,370,900$ & $2.5 \%$ & $1,194,500$ & 0.22 & 0.07 \\
\hline
\end{tabular}

Table 4.9 holds our results for six-player Chinese Checkers. While the ergodic update mechanisms again perform on a level close to each other, $\operatorname{POS}(k)$ performed slightly worse. The best rule that is applicable here is the Transposition rule, with an $8 \%$ reduction in tree size, within $90 \%$ certainty, similar to the results found in the 
previous chapter. Despite their lessened statistical impact, we do see a reduction in tree size from all of them. We thus suspect that there is an improvement from the use of the Threat-ADS, albeit a somewhat small one in this specific scenario. The Stochastic Move-to-Rear, however, has a particularly small impact in this case, as well.

Table 4.9: Average Tree Size for the 6-player Chinese Checkers scenario when the measurement started from a midgame game position.

\begin{tabular}{|c||c||c||c||c||c|}
\hline \hline Update Mech. & Avg. NC & Imp. & Std. Dev. & P-Value & Effect \\
\hline None & $8,167,800$ & - & $2,564,200$ & - & - \\
\hline Move-to-Front & $7,676,900$ & $6 \%$ & $2,282,700$ & 0.18 & 0.30 \\
Transposition & $7,494,300$ & $8 \%$ & $1,669,200$ & 0.09 & 0.26 \\
Move-Ahead- $k$ & $7,644,500$ & $6 \%$ & $1,833,400$ & 0.21 & 0.20 \\
POS $(k)$ & $7,975,200$ & $3 \%$ & $2,187,400$ & 0.44 & 0.08 \\
Stochastic MTR & $8,202,600$ & $-0.5 \%$ & $2,572,900$ & 0.45 & -0.01 \\
\hline
\end{tabular}

Moving on to Focus, Table 4.10 presents our results for the case involving a fiveturn setup. We observe this time, that the Transposition/POS $(k)$ performed noticeably better (the best results were a $19 \%$ improvement) than the Move-to-Front/MoveAhead- $k$. Our results for the Move-to-Front/Move-Ahead- $k$ fell just outside the $95 \%$ certainty, with a P-Value of 0.051, while the improvement for Transposition/POS $(k)$ was statistically significant. We note too that there was a larger Effect Size, and smaller variance in this latter case. While the Stochastic Move-to-Rear did better in this particular case, its benefit still fell outside the bounds of statistical significance.

Lastly, Table 4.11 shows our results for the special three turn case, used in the previous chapter. This time we see that the results are more in line with the previously obtained results, with all the ergodic update mechanisms performing roughly the same and the Stochastic Move-to-Rear rule producing a very small change, and with similar statistical qualities. Our best results come from the Move-to-Front, with a large $19 \%$ improvement, from 16, 767, 600 to $13,592,300$. 
Table 4.10: Average Tree Size for Focus when the measurement started from a midgame game position, with a five-turn setup.

\begin{tabular}{|c||c||c||c||c||c|}
\hline \hline Update Mech. & Avg. NC & Imp. & Std. Dev. & P-Value & Effect \\
\hline None & $14,458,000$ & - & $6,546,500$ & - & - \\
\hline Move-to-Front & $13,050,400$ & $10 \%$ & $9,806,700$ & 0.051 & 0.21 \\
Transposition & $11,780,600$ & $19 \%$ & $6,433,500$ & 0.02 & 0.41 \\
Move-Ahead- $k^{*}$ & $13,050,400 *$ & $10 \%$ & $9,806,700$ & 0.051 & 0.21 \\
POS $(k)^{*}$ & $11,780,600 *$ & $19 \%$ & $6,433,500$ & 0.02 & 0.41 \\
Stochastic MTR & $13,417,600$ & $7 \%$ & $8,095,000$ & 0.10 & 0.16 \\
\hline
\end{tabular}

Table 4.11: Average Tree Size for Focus when the measurement started from a midgame game position, with a three-turn setup.

\begin{tabular}{|c||c||c||c||c||c|}
\hline \hline Update Mech. & Avg. NC & Imp. & Std. Dev. & P-Value & Effect \\
\hline None & $16,767,600$ & - & $7,160,900$ & - & - \\
\hline Move-to-Front & $13,592,300$ & $19 \%$ & $5,234,600$ & 0.01 & 0.44 \\
Transposition & $13,671,500$ & $18 \%$ & $4,235,600$ & 0.03 & 0.43 \\
Move-Ahead- $k^{*}$ & $13,592,300^{*}$ & $19 \%$ & $5,234,600$ & 0.01 & 0.44 \\
POS $(k)^{*}$ & $13,671,500^{*}$ & $18 \%$ & $4,235,600$ & 0.03 & 0.43 \\
Stochastic MTR & $16,226,300$ & $3 \%$ & $6,711,300$ & 0.46 & 0.08 \\
\hline
\end{tabular}

\subsection{Discussion}

The results presented above reinforce our earlier conclusions, demonstrating that the BRS achieves a clear improvement in move ordering by invoking any of a number of ergodic adaptive list techniques, through the Threat-ADS heuristic. Analogous to our previous results, the use of the Threat-ADS generally resulted in a reduction in the tree size as high as $20 \%$, with an average of around $10 \%$. In most cases, all ergodic update mechanisms produced a statistically significant improvement in the tree size. This guarantees that the improvement observed in the previous chapter was not simply a random consequence of the properties of the Move-to-Front strategy. 
We observe that in the majority of cases, the well-known ergodic adaptive list techniques (the Move-to-Front, Transposition, Move-Ahead- $k$, and $\operatorname{POS}(k)$ rules) achieved roughly equal performance, with some small variances occurring in the NC. However, there exist some noticeable exceptions to this. In the case of the 4-ply Virus Game, from the starting position, the best performing update mechanism, $\operatorname{POS}(k)$, in fact generated a statistically significant improvement from the Transposition rule itself. We furthermore remark that in the case of six-player Chinese Checkers from the starting position, as well as four-player Chinese Checkers from the midgame state, the Move-to-Front and $\operatorname{POS}(k)$ rules performed very well.

We note that the Transposition rule, which performed worse in the majority of cases, is the most conservative update mechanism that we investigated, in terms of its changes to the underlying data structure made with each query. We also observe that the Move-Ahead-2, in the case of a six-player game, is similarly more conservative in terms of list changes than either the Move-to-Front or $\operatorname{POS}(2)$. We therefore believe that the results indicate that, especially in the case of Chinese Checkers, more sensitive update mechanisms allow the Threat-ADS heuristic to adapt more quickly to a rapidly changing board position, producing better results than a more conservative strategy. Contrasting this, we observe that the Transposition rule in fact performed better when we consider the case of Focus, starting from a midgame state, with a five-turn setup. We clarify this phenomenon as follows: After five turns into the game of Focus, it is likely that one player has achieved a strong lead, and thus the Transposition rule may be quickly prioritizing him, and thereafter keeping the strong opponent's identity at the front of the list, rather than replacing him as frequently as the Move-to-Front rule does. This leads to a possible paradigm of the system changing the update mechanism employed as the game progresses to a different state.

Unlike the ergodic structures, the Stochastic Move-to-Rear rule did not produce any statistically significant advantage, or disadvantage, compared to the BRS without the use of Threat-ADS. This implies that, at least in the game models examined, convergence to a fixed structure is not advantageous, as relative opponent threat levels tend to be shifting over the course of the game. This phenomenon of the 
Stochastic Move-to-Rear rule reflects our expectations, and we hypothesize that this property will also extend to other absorbing ADSs. However, we reinforce our position that the Threat-ADS is interested in finding the opponent with the strongest board position, which naturally changes over time. Absorbing ADSs may be of use in other, yet-unexplored techniques, that are concerned with finding the opponent who is the most skilled, which represents an alternative conception of opponent threat discussed in Section 3.3.

We did observe slightly better performance from the Stochastic Move-to-Rear rule in the specific case of midgame Focus, with a five-turn setup. As with the Transposition rule, we suspect this may be caused by the fact that five turns into the game, it is likely that a single opponent would have captured several pieces and be close to winning. The Stochastic Move-to-Rear rule may then be converging to place this opponent at the front of the list, and is thus able to glean some benefit.

Finally, from our experiments, we observe that the same reduction in tree size is observed in both 6-ply and 4-ply trees, in the midgame start case. However, in the case of experiments starting from the initial board state, we did, in fact, observe an improvement in tree pruning in the 6-ply case, compared to the 4-ply case. In the Virus Game, at the start of the game, the players may be quite isolated from each other, compared to the midgame state where most squares have been claimed. Therefore, we offer as a possible explanation of this phenomena, that the relative opponent threats at the 6-ply level are very likely the same as at the 4-ply level. Thus, at a 6-ply depth, the Threat-ADS is able to utilize the learning achieved higher in the game tree to a greater effect.

Compared to our results originally reported in [38], we observe that the average NC, when the Threat-ADS is applied, is roughly the same, and that it is, in fact, the BRS without Threat-ADS that performed worse in this work, in a deeper tree. This aside, our results certainly support the conclusion that the Threat-ADS' advantages persist in deeper trees. While we do admit that a 6-ply tree is not what one would consider to be a "very deep" game tree, the exclusion of domain specific heuristics, 
and the highly explosive nature of most multi-player games, makes it infeasible for any analysis to be done in deeper trees. Thus, our conclusion is that there is no noticeable decay in performance as the tree gets deeper, which we believe to be a non-trivial observation, given that it does not hold for other established heuristics. This certainly constitutes a valuable result considering the minimal additional work involved in including an ADS in the game play philosophy. The investigation of the Threat-ADS in deeper game trees, perhaps with access to more powerful hardware resources, remains an avenue that is still unexplored.

\subsection{Chapter Conclusions}

In this chapter, we have attempted to generalize the work presented previously on the behaviour of the Threat-ADS heuristic. We have shown how different ADSbased update mechanisms can produce different results, in terms of the efficacy of the heuristic. Furthermore, we have provided evidence that the Threat-ADS heuristic does not deteriorate as the depth of the search increases. All the results presented have also been accompanied by a formal Mann-Whitney statistical analysis. We have finally demonstrated that, in the case of the Threat-ADS, absorbing ADS paradigms do not appear to be as useful as ergodic ones.

The next chapter will introduce a new method by which game playing, in both multi-player and two-player cases, can be augmented by incorporating ADSs. 


\section{Chapter 5}

\section{History-Based Move Ordering using ADSs}

\subsection{Chapter Overview}

In the previous two chapters, we introduced the Threat-ADS heuristic, which achieves move ordering by ranking opponents using an ADS. Additionally, we analyzed its performance under a wide range of experimental configurations, and verified that it could, generally speaking, achieve a statistically significant improvement in terms of move ordering. We have thus confirmed that the Threat-ADS serves as a strong basis for the application of ADS-based techniques to game playing.

In this chapter, we will expand on these concepts with a new style of move ordering heuristic, which makes use of move history, rather than opponent threat, as its ranking metric. We will show that this technique, which we have named History-ADS, is also able to achieve statistically significant improvements in move ordering, above and beyond what the Threat-ADS could accomplish, and that it can be applied to twoplayer and multi-player games, under a similarly varied set of parameters ${ }^{1}$.

\footnotetext{
${ }^{1}$ The work in this chapter was originally published in [39], and in its journal form in [44].
} 


\subsection{Motivation}

The previous chapters introduced techniques specific to multi-player games, employing the quality of relative opponent threats, ranked through an ADS, to achieve move ordering. We found that our new technique, the Threat-ADS heuristic, was able to achieve statistically significant gains in terms of move ordering through a low-cost application of an ADS.

Given the success of ADS-based techniques within the context of multi-player games, we are now interested in investigating whether they have applications within the context of two-player game playing, as well. While multi-player games represent a relatively unexplored domain, the emphasis in the literature has been on two-player game playing, and this continues to be the case. Thus, the introduction of powerful ADS-based techniques for two-player game playing has the potential to demonstrate the power of applying ADSs to game playing in a much broader context. Furthermore, as the majority of multi-player techniques are derived from two-player counterparts, most two-player techniques involving ADSs would also be applicable in the multiplayer domain.

Given that our proven success with ADS-based techniques is within the context of move ordering, for our first foray into the domain of two-player games, we will again target move ordering as our area for improvement. However, we intuitively cannot make use of relative opponent threats in the two-player context, as there is only a single opponent. Thus, we require a new metric by which move ordering can be accomplished. Here, we turn to the established idea of utilizing knowledge of which moves were earlier determined to be good, so as to accomplish superior move ordering later in the search. This metric, defined here as move history, is our new criterion. The proven efficacy of move ordering schemes in the past, such as in the History heuristic and Killer Moves [54], which make use of such criteria, provide a strong motivation for examining whether ADS-based techniques can effectively use it, or not. 


\subsection{Move Ordering Using Move History}

The idea of ordering moves on the basis of move history is based on an intuitive assumption: If a move is found to be "good" in some location within the game tree, it is likely to also prove to be beneficial elsewhere, and should be explored earlier. For example, within the context of iterative deepening, the well-known idea of ordering moves based on the principal variation of the previous iteration (discussed in Chapter 2 ) is an example of move ordering using move history. If the moves in question were found to be good in the previous iteration, we hypothesize that they will be good in the current iteration, and thus they form the leftmost nodes at each level of the tree.

In this work, however, when speaking about a move being "good", we generally mean that this is a move that had earlier produced either an alpha or beta cut in the tree. Thus, if the same move is encountered elsewhere, our hypothesis is that it is more likely than its peers to produce a cut again, and thus we search it before the others. Well-known move ordering strategies, such as the History heuristic and Killer Moves, also discussed in Chapter 2, operate based on this idea. As the search proceeds, moves which produce a cut are stored in a data structure of some kind, and if they or similar moves are encountered again, they are prioritized according to some criterion.

As a visual demonstration of how tree pruning can be improved through this method, consider first Figure 5.1, where we showcase a fragment of an alpha-beta search tree where the same move produces a cut in different locations, but move ordering based on move history is not employed.

Now, consider Figure 5.2, which shows an equivalent fragment of the search tree, but where move history has been invoked. Observe that the number of moves searched is reduced in the second case. 

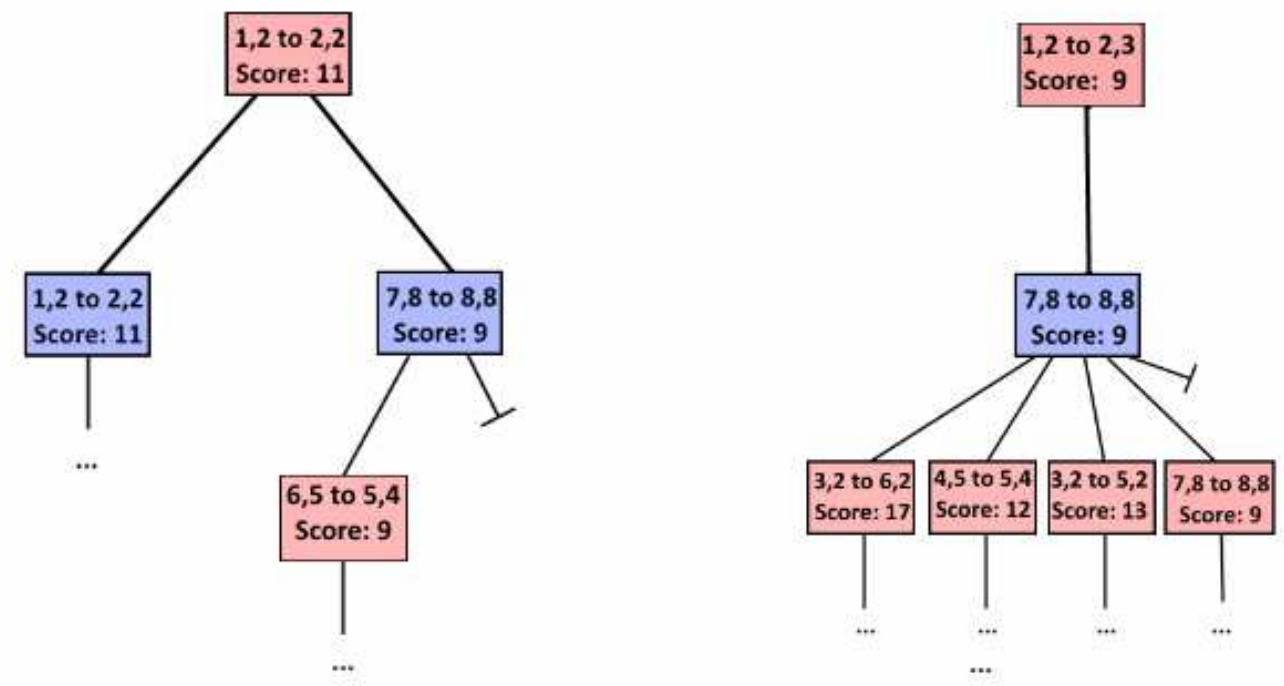

Figure 5.1: An example of an alpha-beta search without including move ordering that is based on move history. Observe that, while the move $(7,8)$ to $(8,8)$ produced a cut earlier in the search, it is not prioritized when it is encountered elsewhere, and thus more nodes are explored.

It is important to recognize that move ordering strategies based on move history can be used in both two-player and multi-player game playing, and thus, as mentioned earlier, the techniques that we develop in this chapter will be applicable to both of these cases.

\subsection{Managing Move History Using an ADS}

We have established that in order to accomplish move ordering through the use of move history, we must somehow record those moves that are "good" (that produce cuts within the tree), and recall them elsewhere when they are encountered again. 

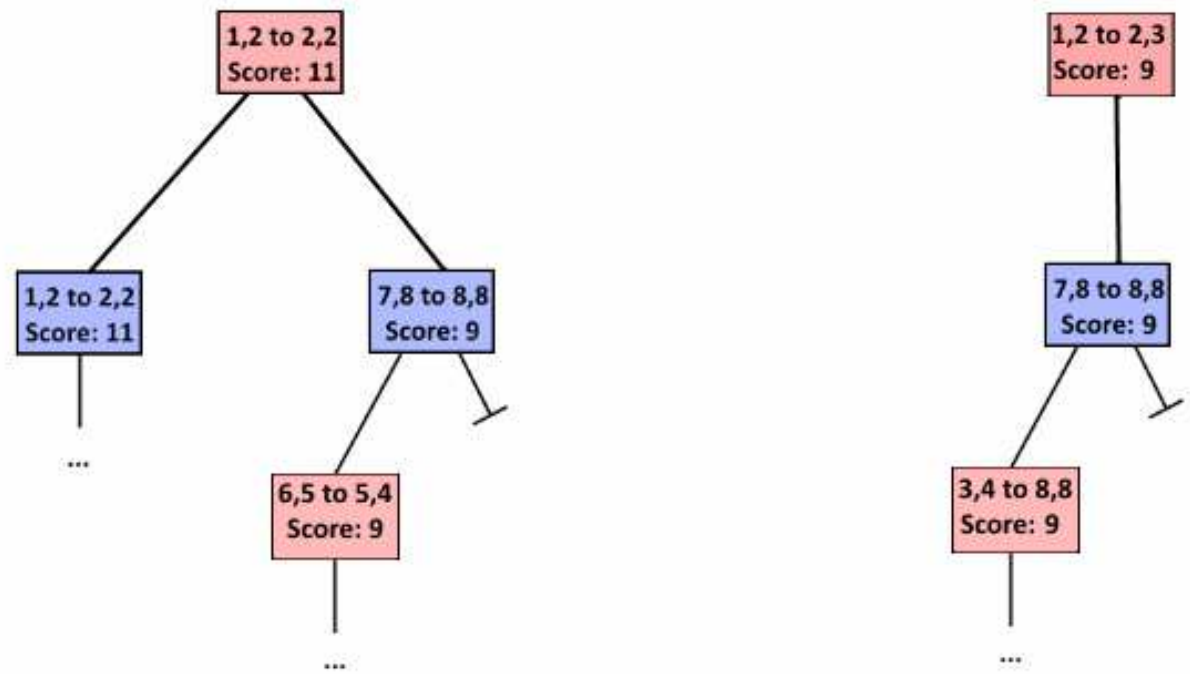

Figure 5.2: An example of an alpha-beta search augmented with move ordering that is based on move history. In this case, the move $(7,8)$ to $(8,8)$ is prioritized somehow, and fewer nodes are explored later in the search.

Furthermore, in the case that at any level of the tree multiple moves are encountered that have previously produced a cut, it would be beneficial to order them in some meaningful way, as opposed to searching them before their neighbors in any arrangement. Lastly, we seek a mechanism by which we record and rank moves that have produced a cut, to be as efficient as possible. We are thus in a situation similar to what we were formerly, in Section 3.4, and again, an ADS provides exactly the sort of qualities we desire.

Rather than utilizing an ADS whose elements are opponents, we, instead, can employ an ADS containing moves. However, unlike the case with the Threat-ADS, we begin with an empty ADS, i.e., of one of those described in Section 2.7. When a move is found to produce a cut, we query the ADS with the identity of that move. To illustrate this, in the case of Chess, we would query it with the co-ordinates of the 
square the piece originated from, and the co-ordinates of its destination, as one does in invoking the History heuristic. If the ADS already contains the move's identity, its position within the ADS is changed according to the ADSs' update mechanisms. If it is not within the ADS, it is instead appended to the end, and immediately moved as if it were queried.

As an example of how the ADS can manage move history over the course of the game, consider Figure 5.3, which showcases its learning process and application over a fragment of the search.
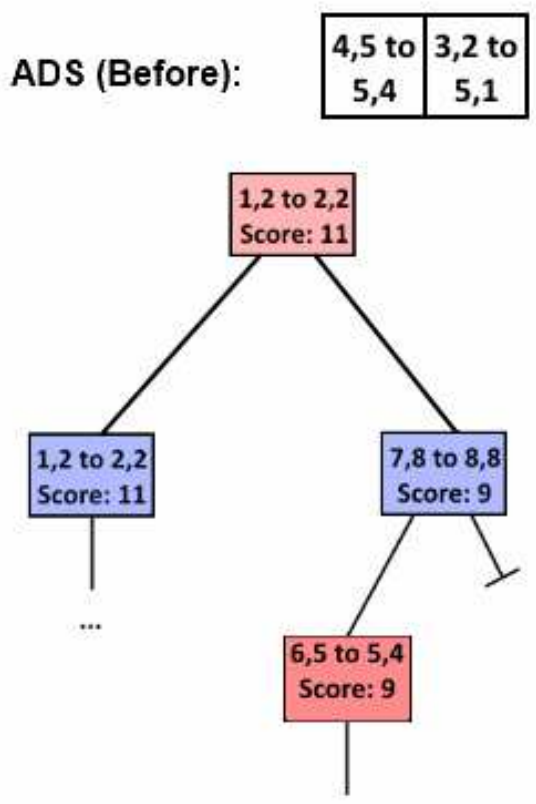

$\cdots$
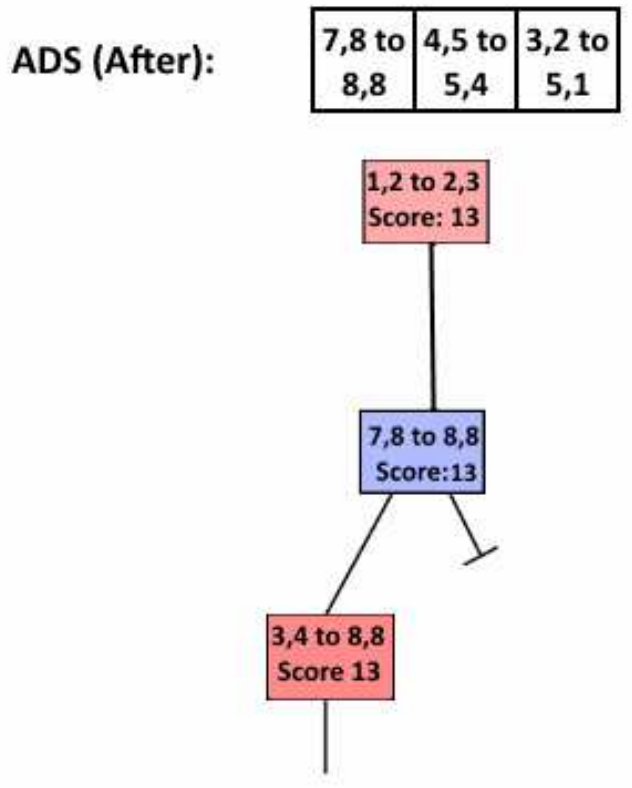

-..

Figure 5.3: A demonstration of how an ADS can be used to manage move history over time. The move $(7,8)$ to $(8,8)$ produces a cut, and so it is moved to the head of the list, and informs the search later.

Unlike in the case of the Threat-ADS, which, at most, contained only a few elements that represented the number of opponents, the ADS in the present setting could contain hundreds of elements, as many moves could produce a cut over the 
course of the search. Thus, there are more possible ways than a simple list to envision this ADS, which is a topic that we intend to explore elsewhere. For the purposes of this chapter, however, we will begin with the conceptually simplest style of ADS, where a single adaptive list simply holds all moves that produce a cut for either the perspective player or the opponent (or opponents, in the BRS case), and it adapts according to its update mechanism.

\subsection{The History-ADS Heuristic}

Having explained how the move history can be used to augment move ordering, and how an ADS can be used to both learn and employ it, we will now detail a formal heuristic, designed in a manner that is analogous to the Threat-ADS in Chapter 3. This heuristic employs the conceptually simple ADS to accomplish move ordering in either a two-player or multi-player game. We refer to this heuristic as the History-ADS heuristic, and its development and features are described in the following sections.

\subsubsection{Developing the History-ADS Heuristic}

To develop the History-ADS heuristic, we must, first of all, understand how to update the ADS at an appropriate juncture, i.e., through querying it with the identity of a move that has performed a cut. This is analogous to querying the ADS with the most threatening opponent within the context of the Threat-ADS. Then, at each Max and Min node, we must specify how we are to order the moves based on the order of the ADS.

Fortunately, within the context of the alpha-beta search, there is a very intuitive location to query the ADS, which is where an alpha or beta cutoff occurs, before terminating that branch of the search. To actually accomplish the move ordering, when we expand a node and gather the available moves, we propose to explore them in the order dictated by the ADS. 
The last issue that must be considered is that, unlike in the Threat-ADS where opponent threats were only relevant on Max nodes, when considering move history, the information is relevant on both Max and Min nodes. Furthermore, we observe that a move that produces a cut on a Max node may not be likely to produce a cut on a Min node, and vice versa. This would occur, for example, if the perspective player and the opponent do not have analogous moves, such as in Chess or Checkers, or if they are some distance from each other. We thus employ two list-based ADSs within the History-ADS heuristic, one of which is used on Max nodes, while the other is used on Min nodes. Algorithm 5.1 shows the Mini-Max algorithm, when it incorporates the History-ADS heuristic.

\subsubsection{Salient Features of the History-ADS Heuristic}

The History-ADS heuristic, in its construction, has some aspects similar to the Threat-ADS heuristic. As with the Threat-ADS, it does not, in any way, alter the final value of the tree, and thus cannot deteriorate the decision-making capabilities of the Mini-Max or BRS algorithms. Similar to the Threat-ADS, the ADSs are added to the search algorithm's memory footprint, and their update mechanisms with regard to its running time.

Compared to the Threat-ADS, the History-ADS can be expected to employ a much larger data structure, as there will be many more possible moves than total opponents in any non-trivial game. Furthermore, as illustrated above, we maintain two separate ADSs in the case of the History-ADS, which rank minimizing and maximizing moves, respectively. The History-ADS thus remembers any move that produces a cut within its ADS for the entire search, even if it never produces a cut again and lingers near the end of the list. Further, we emphasize that new moves could be regularly added to the corresponding lists. However, while this may appear to suggest that the data structures are of unbound size, depending on how we identify a move, there are, in fact, a limited number of moves that can be made within a game. 


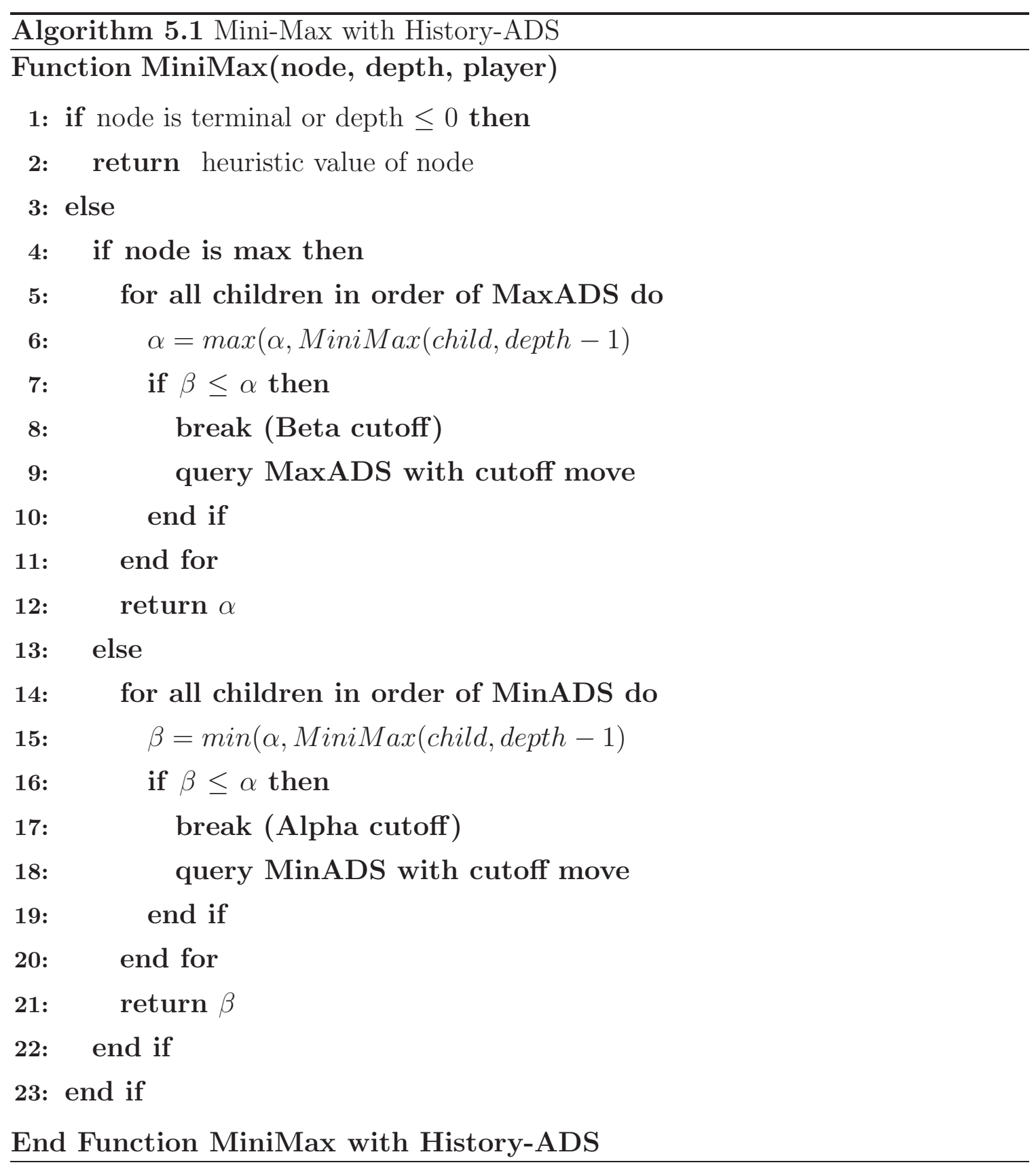


Revisiting the example of Chess, if, as in the case of the History heuristic, we consider a move to be identified by the co-ordinates of the square in which the moved piece originated, and the co-ordinates of its destination square, we see that there are a maximum of 4096 possible moves. This figure serves as an upper bound on the size of the ADS. In the case of the History heuristic, an array of 4096 values, or whichever number is appropriate for the game, is maintained for both minimizing and maximizing moves, whereas the History-ADS only maintains information on those moves that have actually produced a cut. Unarguably, this is a significantly smaller subset. We thus conclude that the memory requirements of the History-ADS are upper bounded by the highly-regarded History heuristic.

Perhaps more importantly, unlike the History heuristic, which requires moves to be sorted based on the values in its arrays, the History-ADS shares the advantageous quality of the Threat-ADS in that it does not require sorting. One can simply explore moves, if applicable, in the order specified by the ADS, thus allowing it to share the strengths of the Killer Moves heuristic, while simultaneously maintaining information on all those moves that have produced a cut.

Lastly, unlike the Threat-ADS, which was specific to the BRS, the History-ADS works within the context of the two-player Mini-Max algorithm. However, since the BRS views a multi-player game as a two-player game by virtue of it treating the opponents as a single entity, the History-ADS heuristic is also applicable to it, and it thus functions in both two-player and multi-player contexts. We will thus be investigating its performance in both these avenues in the following sections.

\subsection{Experimental Model}

As the two heuristics share many conceptual commonalities, we consider it useful to employ a similar set of experiments in analyzing the performance of the History-ADS heuristic (as in Chapters 3 and 4), so as to allow the performance of the heuristics to be more easily compared. However, as our work presented in this chapter applies to both 
the two-player and multi-player cases, and since we desire to improve the execution time of the experiments, we have opted to not use precisely the same software system or configuration. Thus, we will not be directly using any values presented in the previous chapters, in order to mitigate any factors that could influence the respective performance in an unforseen way.

As before, we are interested in learning the improvement gained from using the History-ADS heuristic, when compared to a search that does not employ it. Thus, we accomplish this by taking an aggregate of the NC over several turns, which we then average over fifty trials. We will repeat this experiment with a variety of games, with different ADS update mechanisms, and at varying ply depths, so as to provide us information about the History-ADS, its benefits and drawbacks, and its overall efficacy.

As in the previous chapters, we will employ the Virus Game, Focus, and Chinese Checkers when considering the multi-player case. This will allow our results for the History-ADS to be more easily compared with our results for the Threat-ADS. However, since we are also considering the two-player case, we require an expanded testing set of games. While the Virus Game, Focus, and Chinese Checkers can all be played with two players, we have elected to employ some more well-known two-player games, rather than using the same games in their two-player configurations. This is done so as to provide a wider testing base for the History-ADS, which will be used in future chapters as well. The new two-player games that we will employ are Othello, which will be described in some detail in Appendix A, and the very well-known Checkers, or Draughts. However, the requirement in Checkers that forces jumps when possible, often leads to a game with a very small branching factor. This factor can vary greatly especially in different midgame states. Thus, for our experiments, we choose to relax this rule, and do not require that a player must necessarily make an available jump. We shall refer to this game as "Relaxed Checkers". Lastly, we will use Focus in both the two-player and multi-player cases. 
In testing the Threat-ADS heuristic, we had varied the update mechanism used, the ply depth of the search, and the number of players, as well as beginning the experiments at the initial board position, and a midgame position. As the HistoryADS is an entirely new heuristic, it is desirable to test it with the same number of parameters as with the Threat-ADS. However, testing all possible combinations would have an explosive effect on the number of experiments, and clutter our results with redundant or uninteresting cases. Therefore, based upon our results from previous chapters, we opt to select a subset of the possible number of experiments, i.e., selecting those cases that are most likely to have an impact on the performance of the HistoryADS.

With regard to the ADSs themselves, rather than test the complete set of ergodic updating mechanisms, we choose the extremes of the Move-to-Front and Transposition rules, omitting the $\operatorname{POS}(k)$ and Move-Ahead- $k$ rules, which serve as compromises between them. Further, we do not include the Stochastic Move-to-Rear, given its previous failure. Given the execution time of the experiments, reported previously, we limit our experiments using Focus and Chinese Checkers to a 4-ply search depth. However, as in Chapter 4, we test at both a 4-ply and 6-ply depth in the case of the Virus Game. In the case of the new two-player games, they generally have a much smaller branching factor, and so we test them at 4-ply, 6-ply, and 8-ply search depths. Lastly, as the History-ADS deals with moves rather than players, we maintain the number of players in multi-player games to be four, with the exception of Chinese Checkers, where we test six as well, given the difference in performance noted earlier between those cases.

All of our experiments will again be performed from an initial board state, summing the total $\mathrm{NC}$ over the first few moves of the game, and starting from a midgame board state, recorded using the same method described in the preceding chapters. We take measurements over the same number of turns for each of the games used in the previous chapters, that is, ten for the Virus Game, five for Chinese Checkers, and three for Focus. Finally, when considering a midgame board state, we allow them to run for the same number of turns before taking measurements, which is fifteen 
for the Virus Game, ten for Chinese Checkers, and five for Focus. For Othello and Relaxed Checkers, we decide to take measurements over five turns for each of them, and advance the game five turns for Relaxed Checkers, and ten for Othello, before taking measurements in the midgame case.

As in previous chapters, we do not make the assumption of normalcy, and employ the Mann-Whitney test to determine the statistical significance, as well as providing the Effect Size metric to analyze our results.

As before, all our experiments are run on an Intel i5 $3.4 \mathrm{GHz}$ processor. Our results are presented in the next section.

\subsection{Results for Two-Player Games}

First, consider Table 5.1, where we present our results for the game Othello. We observe a consistent pattern for all ply depths, as well as initial and midgame starting positions. In all cases, the History-ADS heuristic generated a noticeable improvement in terms of NC, and the Move-to-Front update mechanism consistently outperformed the Transposition rule. The degree of improvement, in terms of percentage, was also consistently larger when the tree was larger, either due to a greater branching factor in the midgame state, or a deeper search, with the best improvement being a substantial $47 \%$, starting from a midgame position, at an 8-ply depth, using the Move-to-Front rule, from 182, 500 to 95, 600. This is, by all descriptions, a substantial improvement, which is supported by our test for significance. While the Move-toFront rule outperformed the Transposition rule in all cases, the difference between the two was not statistically significant to a $95 \%$ certainty threshold in any case. Effect Sizes ranged between 0.5 and 0.8, with a larger Effect Size roughly corresponding to a larger improvement. 
Table 5.1: Results of applying the History-ADS heuristic for Othello in various configurations.

\begin{tabular}{|c||c||c||c||c||c||c||c|}
\hline \hline Ply & Mid & Update Mech. & Avg. NC & Imp. & Std. Dev. & P-Value & Effect \\
\hline 4 & No & None & 669 & - & 205 & - & - \\
4 & No & Move-to-Front & 523 & $22 \%$ & 162 & $2.2 \times 10^{-4}$ & 0.71 \\
4 & No & Transposition & 572 & $15 \%$ & 225 & 0.016 & 0.47 \\
\hline 6 & No & None & 5061 & - & 2385 & - & - \\
6 & No & Move-to-Front & 3827 & $24 \%$ & 1692 & $6.0 \times 10^{-3}$ & 0.51 \\
6 & No & Transposition & 4057 & $20 \%$ & 2096 & 0.015 & 0.42 \\
\hline 8 & No & None & 38,800 & - & 20,300 & - & - \\
8 & No & Move-to-Front & 26,800 & $30 \%$ & 12,000 & $1.2 \times 10^{-3}$ & 0.59 \\
8 & No & Transposition & 29,700 & $23 \%$ & 12,300 & 0.014 & 0.45 \\
\hline \hline 4 & Yes & None & 2199 & - & 745 & - & - \\
4 & Yes & Move-to-Front & 1699 & $23 \%$ & 633 & $2.2 \times 10^{-3}$ & 0.67 \\
4 & Yes & Transposition & 1761 & $20 \%$ & 597 & 0.01 & 0.59 \\
\hline 6 & Yes & None & 20,100 & - & 9899 & - & - \\
6 & Yes & Move-to-Front & 14,500 & $28 \%$ & 6303 & $6.0 \times 10^{-3}$ & 0.57 \\
6 & Yes & Transposition & 15,200 & $24 \%$ & 6751 & 0.015 & 0.49 \\
\hline 8 & Yes & None & 182,500 & - & 114,000 & - & - \\
8 & Yes & Move-to-Front & 95,600 & $47 \%$ & 50,200 & $<1.0 \times 10^{-5}$ & 0.76 \\
8 & Yes & Transposition & 113,700 & $38 \%$ & 60,900 & $1.3 \times 10^{-4}$ & 0.60 \\
\hline
\end{tabular}

Table 5.2 presents our results for Relaxed Checkers. We observe very similar patterns in this case, compared to Othello, where the History-ADS always generated a noticeable improvement, although to a much higher level of statistical certainty. This improvement was usually greater when the tree was larger. Effect Sizes were larger, compared to Othello, particularly in the case of the initial board state, with the highest measure being a very large 3.58. Again, the Move-to-Front rule always outperformed the Transposition rule when all other parameters were kept equal, and the difference between the two was found to be statistically significant itself in all 
cases. Our best improvement in this case was a reduction of the tree size of $63 \%$, and this occurred in the 8-ply case, starting from a midgame position and using the Move-to-Front update mechanism, representing a reduction in NC from 859, 200 to 317,100 .

Table 5.2: Results of applying the History-ADS heuristic to Relaxed Checkers in various configurations.

\begin{tabular}{|c||c||c||c||c||c||c||c|}
\hline \hline Ply & Mid & Update Mech. & Avg. NC & Imp. & Std. Dev. & P-Value & Effect \\
\hline 4 & No & None & 5930 & - & 864 & - & - \\
4 & No & Move-to-Front & 4712 & $20 \%$ & 461 & $<1.0 \times 10^{-5}$ & 1.41 \\
4 & No & Transposition & 5148 & $13 \%$ & 675 & $<1.0 \times 10^{-5}$ & 0.90 \\
\hline 6 & No & None & 78,600 & - & 10,600 & - & - \\
6 & No & Move-to-Front & 40,800 & $48 \%$ & 5619 & $<1.0 \times 10^{-5}$ & 3.58 \\
6 & No & Transposition & 48,600 & $38 \%$ & 6553 & $<1.0 \times 10^{-5}$ & 2.84 \\
\hline 8 & No & None & 910,300 & - & 172,400 & - & - \\
8 & No & Move-to-Front & 361,800 & $60 \%$ & 55,900 & $<1.0 \times 10^{-5}$ & 3.18 \\
8 & No & Transposition & 435,000 & $52 \%$ & 68,400 & $<1.0 \times 10^{-5}$ & 2.76 \\
\hline \hline 4 & Yes & None & 5447 & - & 1859 & - & - \\
4 & Yes & Move-to-Front & 3772 & $31 \%$ & 1257 & $<1.0 \times 10^{-5}$ & 0.90 \\
4 & Yes & Transposition & 4497 & $17 \%$ & 1474 & $4.5 \times 10^{-3}$ & 0.51 \\
\hline 6 & Yes & None & 64,000 & - & 25,700 & - & - \\
6 & Yes & Move-to-Front & 36,100 & $44 \%$ & 12,700 & $<1.0 \times 10^{-5}$ & 1.08 \\
6 & Yes & Transposition & 43,600 & $32 \%$ & 16,600 & $<1.0 \times 10^{-5}$ & 0.79 \\
\hline 8 & Yes & None & 859,200 & - & 408,500 & - & - \\
8 & Yes & Move-to-Front & 317,100 & $63 \%$ & 134,600 & $<1.0 \times 10^{-5}$ & 1.33 \\
8 & Yes & Transposition & 422,200 & $51 \%$ & 160,600 & $<1.0 \times 10^{-5}$ & 1.07 \\
\hline
\end{tabular}

Our results for Focus are presented in Table 5.3. Again, as in the case of the previous two games, we see a similar pattern emerge, but even stronger than in the case of Relaxed Checkers, with an extraordinarily high 10.39 Effect Size in the case of 
Move-to-Front, from the initial board position. The Move-to-Front rule outperformed the Transposition rule, and, as Focus has a much larger branching factor than either Othello or Relaxed Checkers, the improvement noted is much greater, and better in the midgame case. The difference between the two update mechanisms was statistically significant itself, again. The best improvement was $77 \%$, in the midgame case, using the Move-to-Front rule, from 10,602, 200 nodes to 2, 417, 000 nodes.

Table 5.3: Results of applying the History-ADS heuristic to two-player Focus in initial and midgame states.

\begin{tabular}{|c||c||c||c||c||c||c||c|}
\hline \hline Ply & Mid & Update Mech. & Avg. NC & Imp. & Std. Dev. & P-Value & Effect \\
\hline 4 & No & None & $5,254,700$ & - & 381,400 & - & - \\
4 & No & Move-to-Front & $1,290,700$ & $75 \%$ & 88,000 & $<1.0 \times 10^{-5}$ & 10.39 \\
4 & No & Transposition & $1,795,700$ & $66 \%$ & 157,900 & $<1.0 \times 10^{-5}$ & 9.07 \\
\hline \hline 4 & Yes & None & $10,602,200$ & - & $3,466,600$ & - & - \\
4 & Yes & Move-to-Front & $2,417,000$ & $77 \%$ & 636,700 & $<1.0 \times 10^{-5}$ & 2.37 \\
4 & Yes & Transposition & $2,914,700$ & $73 \%$ & 760,400 & $<1.0 \times 10^{-5}$ & 2.22 \\
\hline
\end{tabular}

\subsection{Results for Multi-Player Games}

Consider Table 5.4, where we present our results for the Virus Game. We observe very similar trends to those observed in the two-player environment, where the Moveto-Front rule outperformed the Transposition rule, and the savings were generally greater as the size of the tree increases. The best savings was $55 \%$ in the 6-ply case from the initial board state, using the Move-to-Front rule, from 10, 463, 700 to 4,693, 900. Although the Move-to-Front rule did outperform the Transposition rule in all experiments, the difference between the two was, in the case of the Virus Game, not statistically significant. The Effect Size varies between 2.6 and 4.6, showing a very strong impact of the History-ADS. 
Table 5.4: Results of applying the History-ADS heuristic to the Virus Game in various configurations.

\begin{tabular}{|c||c||c||c||c||c||c||c|}
\hline \hline Ply & Mid & Update Mech. & Avg. NC & Imp. & Std. Dev. & P-Value & Effect \\
\hline 4 & No & None & 253,600 & - & 28,600 & - & - \\
4 & No & Move-to-Front & 157,000 & $38 \%$ & 17,900 & $<1.0 \times 10^{-5}$ & 3.37 \\
4 & No & Transposition & 164,600 & $35 \%$ & 22,800 & $<1.0 \times 10^{-5}$ & 3.11 \\
\hline 6 & No & None & $10,463,700$ & - & $1,261,700$ & - & - \\
6 & No & Move-to-Front & $4,693,900$ & $55 \%$ & $1,012,100$ & $<1.0 \times 10^{-5}$ & 4.57 \\
6 & No & Transposition & $4,851,100$ & $54 \%$ & 738,700 & $<1.0 \times 10^{-5}$ & 4.45 \\
\hline \hline 4 & Yes & None & 308,800 & - & 40,700 & - & - \\
4 & Yes & Move-to-Front & 187,800 & $41 \%$ & 17,800 & $<1.0 \times 10^{-5}$ & 2.97 \\
4 & Yes & Transposition & 199,300 & $36 \%$ & 20,700 & $<1.0 \times 10^{-5}$ & 2.69 \\
\hline 6 & Yes & None & $12,801,300$ & - & $1,952,500$ & - & - \\
6 & Yes & Move-to-Front & $5,940,000$ & $54 \%$ & 831,700 & $<1.0 \times 10^{-5}$ & 3.51 \\
6 & Yes & Transposition & $6,063,900$ & $53 \%$ & 974,500 & $<1.0 \times 10^{-5}$ & 3.45 \\
\hline
\end{tabular}

Table 5.5 holds our results for multi-player Focus. Our results are very similar again to the two-player case, with particularly large savings, although the Effect Size is smaller, likely due to the large variance.. Our best result was a very large $78 \%$ reduction in tree size from invoking the Move-to-Front rule, and from the midgame board state, from 14,192, 200 to 3, 236,800. In this case, the Move-to-Front rule's improvement over Transposition was only statistically significant in the initial case.

Finally, Table 5.6 contains our results for Chinese Checkers. The performance in this case followed slightly different patterns than our other experiments. While the Move-to-Front rule consistently outperformed the Transposition rule, the difference was not as pronounced in this case. Furthermore, our best performance was a $65 \%$ reduction from the Move-to-Front in the four player case, from the initial board state, which is the smallest tree, as opposed to the largest. The possible reasons for this observation will be discussed presently. Effect Sizes ranged between 1.75 and 2.2. 
Table 5.5: Results of applying the History-ADS heuristic to multi-player Focus in initial and midgame cases.

\begin{tabular}{|c||c||c||c||c||c||c||c|}
\hline \hline Ply & Mid & Update Mech. & Avg. NC & Imp. & Std. Dev. & P-Value & Effect \\
\hline 4 & No & None & $6,973,500$ & - & 981,000 & - & - \\
4 & No & Move-to-Front & $2,183,100$ & $69 \%$ & 183,900 & $<1.0 \times 10^{-5}$ & 4.88 \\
4 & No & Transposition & $2,737,900$ & $61 \%$ & 271,200 & $<1.0 \times 10^{-5}$ & 4.32 \\
\hline \hline 4 & Yes & None & $14,192,200$ & - & $8,400,200$ & - & - \\
4 & Yes & Move-to-Front & $3,236,800$ & $78 \%$ & $1,728,600$ & $<1.0 \times 10^{-5}$ & 1.30 \\
4 & Yes & Transposition & $3,566,700$ & $75 \%$ & $1,863,800$ & $<1.0 \times 10^{-5}$ & 1.26 \\
\hline
\end{tabular}

\subsection{Discussion}

Our results presented in the previous sections strongly reinforce the hypothesis that an ADS managing the move history, employed by the History-ADS heuristic, can achieve improvements in tree pruning through better move ordering, in both two-player and multi-player games. This confirms that such an ADS does, indeed, correctly prioritize the most effective moves, based on their previous performance elsewhere in the tree, as initially hypothesized.

Our results confirm that the History-ADS heuristic is able to achieve a statistically significant reduction in NC in three two-player games, Othello, Relaxed Checkers, and the two-player variant of Focus, as well as three multi-player games, the Virus Game, Chinese Checkers, and the multi-player variant of Focus employed in the testing of the Threat-ADS. Unlike with the Threat-ADS, where the degree of reduction ranged between $5 \%$ and $20 \%$, and which centered around $10 \%$, we observe in this case a drastic variation in the reduction between cases, i.e., from $13 \%$ to a value as high as $77 \%$, depending on the game and the depth of the search. Despite the wider range, the overall savings are certainly larger when the History-ADS is employed, compared to the Threat-ADS, in the cases where both strategies are applicable. 
Table 5.6: Results of applying the History-ADS heuristic to Chinese Checkers in various configurations.

\begin{tabular}{|c||c||c||c||c||c||c||c|}
\hline \hline Ply & Mid & U. Mech. & Avg. NC & Imp. & Std. Dev. & P-Value & Effect \\
\hline 4 (4-play) & No & None & $1,376,800$ & - & 416,800 & - & - \\
4 (4-play) & No & MtF & 485,500 & $65 \%$ & 135,400 & $<1.0 \times 10^{-5}$ & 2.14 \\
4 (4-play) & No & Transp. & 504,800 & $63 \%$ & 130,800 & $<1.0 \times 10^{-5}$ & 2.09 \\
\hline 4 (6-play) & No & None & $3,366,200$ & - & $1,097,500$ & - & - \\
4 (6-play) & No & MtF & $1,254,200$ & $63 \%$ & 316,200 & $<1.0 \times 10^{-5}$ & 1.92 \\
4 (6-play) & No & Transp. & $1,316,300$ & $61 \%$ & 338,100 & $<1.0 \times 10^{-5}$ & 1.87 \\
\hline \hline 4 (4-play) & Yes & None & $3,340,200$ & - & 933,000 & - & - \\
4 (4-play) & Yes & MtF & $1,313,600$ & $61 \%$ & 365,300 & $<1.0 \times 10^{-5}$ & 2.17 \\
4 (4-play) & Yes & Transp. & $1,360,900$ & $59 \%$ & 313,900 & $<1.0 \times 10^{-5}$ & 2.12 \\
\hline 4 (6-play) & Yes & None & $8,259,600$ & - & $2,640,800$ & - & - \\
4 (6-play) & Yes & MtF & $3,401,100$ & $59 \%$ & 899,300 & $<1.0 \times 10^{-5}$ & 1.84 \\
4 (6-play) & Yes & Transp. & $3,654,100$ & $56 \%$ & 919,800 & $<1.0 \times 10^{-5}$ & 1.74 \\
\hline
\end{tabular}

Our second observation is that, unlike in the previous chapter, where the best update mechanism for the Threat-ADS was different between cases, in this case we observe that in all cases, the Move-to-Front rule outperformed the Transposition rule, and in many cases, generally corresponding to larger overall savings. This is a reasonable outcome, as unlike with the Threat-ADS, the adaptive list may contain dozens to hundreds of elements, and it would take quite a bit of time for the Transposition rule to migrate a particularly strong move to the head of the list. As opposed to this, the Move-to-Front would migrate the move to the front quickly, and would likely keep it there. This phenomenon also confirms that the order of elements within the list matters to the move ordering. In other words, merely maintaining an unsorted collection of moves that have produced a cut will not perform as well as employing an adaptive list, further supporting the use of the History-ADS heuristic. 
When considering the wide range of savings between the various cases, we observe that in general, the larger the overall game tree, the greater are the proportional savings. There are a number of possible reasons why this is the case. Firstly, we note that the History-ADS retains information on all moves that produce a cut, and in a larger tree, it is likely that there will be more moves of this nature. Thus, the History-ADS has, available to it, more information to work with. Secondly, in games with a very large branching factor, such as Focus or Chinese Checkers, many of the available moves tend not to be very good, and the prioritization of a small number of strong moves can thus cut off huge "chunks" of the search space. Lastly, in the case of deeper trees, the History-ADS has more opportunities to apply its knowledge of strong moves gleaned from other levels of the tree, similar to the reasoning behind the original History heuristic.

The exception to the above trend occurs in the case of Chinese Checkers. While Chinese Checkers, with its large branching factor, usually sees a larger reduction in tree size compared to Othello or Relaxed Checkers, savings for all the cases within the context of Chinese Checkers are roughly equivalent. Furthermore, for the the four player case, with measurements being taken from the initial board position, we observe the best performance, despite having the smallest overall tree size. What this suggests is that moves that produce a cut in Chinese Checkers may not have a very strong natural ranking between them, and so attempting to rank them in the ADS does not help as much as it does for the other games. This would also explain why the Move-to-Front rule did not outperform the Transposition rule to the same extent as in the other cases.

While the system employed is slightly different from the one used to test the Threat-ADS, much of the same code was employed, and the reader can clearly see that the values for the BRS without the History-ADS roughly corresponded to the equivalent values in the previous chapters, if one made a rough comparison with the performance of the Threat-ADS. Thus, it appears as though the History-ADS outperforms the Threat-ADS to an extreme extent. For example, in the case of the four-player Virus Game, from the initial board position, the Threat-ADS using the 
Move-to-Front rule generates a $10 \%$ reduction in tree size. As opposed to this, the History-ADS generates a $38 \%$ reduction under the same configuration. This is not particularly surprising, however, as there are many more possible moves than opponents, and so the History-ADS achieves that improved saving with a larger adaptive list. That the Threat-ADS obtains a quarter of the History-ADS' savings with a list of size three is, in and of itself, impressive. Indeed, it remains a viable option in cases where the more complex History-ADS is deemed unnecessary.

\subsection{Chapter Conclusions}

In this chapter, we introduced the History-ADS heuristic, which employs concepts introduced by the Threat-ADS heuristic to order moves, rather than opponents, using an ADS. The History-ADS heuristic ranks moves based on how recently in the tree they produced a cut, similar to the established Killer Moves heuristic and the History heuristic. Unlike the Threat-ADS, the History-ADS is applicable to both two-player and multi-player games, and was shown to achieve statistically significant improvements in tree pruning in a range of games in both domains, and to a noticeably greater degree than the Threat-ADS.

Although the History-ADS performs very well in a variety of situations, it makes use of a conceptually very simple ADS to rank the moves. It simply places all moves that produce a cut into an ADS, and performs the same update whenever a cut occurs, regardless of the level of tree at which the cut exists. As discussed in Chapter 2 , this tends to disproportionately favour moves that produce a cut deep into the tree, and both the Killer Moves and History heuristics contain mechanisms to mitigate it. A currently open question is whether an ADS strategy like the History-ADS can be created that also attempts to handle this, and if it can improve on the performance of the History-ADS.

The next chapter will deal with the construction of such a heuristic, based on the popular Killer Moves heuristic. 


\section{Chapter 6}

\section{Refinements of the History-ADS Heuristic}

\subsection{Chapter Overview}

In the previous chapter, we introduced the idea of applying ADS-based techniques to two-player and multi-player games, employing the move history metric to achieve move ordering. Based on these concepts, we developed the History-ADS heuristic, an approach that was analogous to the Threat-ADS heuristic, employing move history rather than opponent threat as the ordering criterion. We showed that under a wide range of game models and experimental configurations, the History-ADS heuristic was able to obtain substantial gains in terms of tree pruning.

In this chapter, we explore alternative implementation strategies to refine the History-ADS heuristic, to reduce its cost, and to explore potential avenues for improving its performance in various cases ${ }^{1}$.

\footnotetext{
${ }^{1}$ The work in this chapter was originally published in [41], and in its journal form in [44].
} 


\subsection{Open Questions}

We already know that an ADS-based move ordering strategy could obtain significant benefits when move history is employed. Indeed, the History-ADS heuristic could obtain more than a $75 \%$ reduction in tree sizes in some cases. Unfortunately, the History-ADS heuristic, as described earlier, employs only the naive approach of maintaining a single adaptive list, that holds all moves. The exploration of more refined models and strategies remains an open question.

The first possible refinement we consider is that of restricting the maximum size of the ADS. The reader should observe that all moves that create a cut are stored within the ADS employed by the History-ADS heuristic, even if they never produce a cut again. As a result of this, the length of the adaptive list used by the History-ADS heuristic is bounded by the number of possible moves in the game, which could potentially be a very large number. However, it may be the case that, if we utilized a list that is artificially bounded, we could observe only a marginally-reduced performance, while simultaneously drastically decreasing the memory footprint of the History-ADS heuristic.

The second avenue of research is inspired by the History and Killer Moves heuristics, discussed in Chapter 2. In both cases, moves that can found to be good at a specific level of the tree can be sometimes recognized as being potentially poor at other levels, and it may thus not be beneficial to prioritize them. The History-ADS heuristic considers all levels of the tree to be equally important, and thus a separate refinement would be to consider if the History-ADS can benefit from considering the level at which a move produced a cut.

The above motivates the work done in this chapter. 


\subsection{Restricting the Length of the ADS}

This section describes our investigation into providing a bound on the maximum size of the list used by the History-ADS heuristic.

\subsubsection{Strategy and Technique}

To initiate discussions, consider the fact that the History-ADS heuristic updates its ADS whenever a move is found to produce a cut, using the identity of that move. Its position is either changed according to the chosen ADS update mechanism if it is already in the list, or it is appended to the end of the list, after which its position is immediately updated. Thus, any element that produces a cut remains in the list, and it is never pruned, potentially leading to a very large data structure.

Retaining all the information pertaining to moves that have produced a cut is logically beneficial. However, it is possible, and in fact very reasonable to hypothesize, that the majority of savings do not come from moves which are near the tail of the list, but rather near the front. Therefore, if we provide a maximum size on the list, and only retain elements in those positions, it may be possible to noticeably curtail the size of the list, providing some guarantees on its memory performance, while maintaining the vast majority of savings provided by the History-ADS. The way in which we will accomplish this is by forgetting any element of the list that falls to position $N+1$, if the maximum is $N$. Otherwise, the History-ADS will operate as it did in the previous chapter. An example of such an ADS updating over several queries, operating with a bounded list, is presented in Figure 6.1.

Beyond limiting the memory usage of the History-ADS heuristic, if the developer is attempting to avoid sorting moves by generating them in the order of the ADS, as described in the previous chapter, having to traverse a very long list to do this could defeat the purpose of omitting sorting. Thus, demonstrating that the History-ADS achieves good results with a smaller list, mitigates implementation concerns, as well. 


\begin{tabular}{|c|c|c|c|}
\hline 7,8 to & 4,5 to & 3,2 to & 6,7 to \\
8,8 & 5,4 & 5,1 & 6,6 \\
\hline
\end{tabular}

\begin{tabular}{|c|c|c|c|c|}
\hline 1,3 to & 7,8 to & 4,5 to & 3,2 to & 6,7 to \\
1,6 & 8,8 & 5,4 & 5,1 & 6,6 \\
\hline
\end{tabular}

\begin{tabular}{|c|c|c|c|c|c|}
\hline 2,1 to & 1,3 to & 7,8 to & 4,5 to & 3,2 to & 6,7 to \\
2,2 & 1,6 & 8,8 & 5,4 & 5,1 & 6,6 \\
\hline
\end{tabular}

\begin{tabular}{|c|c|c|c|c|}
\hline 3,2 to & 2,1 to & 1,3 to & 7,8 to & 4,5 to \\
5,1 & 2,2 & 1,6 & 8,8 & 5,4 \\
\hline
\end{tabular}

\begin{tabular}{|c|c|c|c|c|c|}
\hline 1,1 to & 3,2 to & 2,1 to & 1,3 to & 7,8 to & 4,5 to \\
1,2 & 5,1 & 2,2 & 1,6 & 8,8 & 5,4 \\
\hline
\end{tabular}

Figure 6.1: An example of a History-ADS's list sequence updating over several queries, with a maximum length of 5 . The ADS starts with a list of length four, and is queried with the move $(1,3)$ to $(1,6)$, which it moves to the front. It is then queried with $(2,1)$ to $(2,2)$, and as $(6,7)$ to $(6,6)$ is pushed to the sixth position, it is forgotten (highlighted in grey). The process continues as it is queried with $(3,2)$ to $(5,1)$, causing only an internal change, and finally $(1,1)$ to $(1,2)$, pushing $(4,5)$ to $(5,4)$ off the end of the list.

\subsubsection{Experimental Model}

In the interest of continuity, we employ the same software system developed to originally test the History-ADS heuristic, extending the implementation of the heuristic to allow for limitations on the maximum length of the list. As the code is otherwise unchanged, we maintain the values presented in the previous chapter for cases where the Mini-Max or Best-Reply Search algorithms were used without the HistoryADS heuristic. We furthermore execute a very similar set of trials to those originally performed to validate the History-ADS. 
In the previous chapter, we tested the Move-to-Front and Transposition rules, and found strong evidence that, in the case of the History-ADS heuristic, the Move-toFront update mechanism strongly outperforms the Transposition rule. As our focus in this chapter is on the maximum length and the multi-level ADSs, we limit the update mechanism to only the Move-to-Front rule. Aside from that, our experimental configuration is identical to that presented in the previous chapter, taking the aggregate $\mathrm{NC}$ over the same number of turns as before, with the same games and parameters as in that chapter. Furthermore, the relevant measurements are taken from both the initial board position and the midgame state ${ }^{2}$.

When considering limitations on the maximum length of the ADS, we repeat the experiments for an unbounded list (analogous to the Move-to-Front results from the previous chapter), and with lists bounded at lengths of 5 and 20. From the perspective of our study, we recognize that cases where the maximum length is substantially larger than 20 are not meaningful, given that most games will not fill a list of this length during the execution of the search. At the upper level, the list length of 20 is therefore a value that allows a substantial amount of information to be recalled, while providing a limit that the search will reach, and may thus have an effect on its performance. On the other hand, on the lower level, the list length of 5 is chosen because with a limit of 5, the History-ADS heuristic's adaptive list length is more in line with the very short list employed by the Threat-ADS.

As before, our analysis includes the Mann-Whitney test to determine the statistical significance, and the Effect Size metric. We employ the same Intel i5 $3.4 \mathrm{GHz}$ processor as in the previous chapter. Our results are presented in the next section.

\footnotetext{
${ }^{2}$ To avoid unnecessary repetition, the reader is referred to the previous chapter for the precise configuration of these experiments and the games employed.
} 


\subsubsection{Results for Bounded ADSs}

Our results for Othello with limits on the ADS' size are presented in Table 6.1. As expected, we observed similar improvements gained from the History-ADS as we did in the previous chapter, and in each case the improvement remains statistically significant. The improvement was observably lessened when the ADS is capped, and a stricter limit on the length resulted in a lesser reduction in the Node Count. This was consistent across all cases. In a very encouraging scenario, the decrease was by only $1 \%$ (from $26 \%$ to $25 \%$, a change of 3727 to 3779 , from a result of 5061 employing no ADS), when the ply depth was 6 and the size of the list was bounded by 20, from the initial board position. Those results marked with a "*" represent cases where the difference with an unbound list was itself statistically significant. For Othello, these were the 4-ply case from the initial board state, and when the search depth is 8-ply at both initial and midgame states.

Table 6.2 presents our results for Checkers. A similar pattern was observed as in the case of Othello, where the smaller the length of the list, the less the improvement gleaned from the History-ADS heuristic. In the very best case, the reduction in savings was only $2 \%$, when the size of the list was bounded by 20 , from the initial board state (regardless of ply depth). As before, all invocations of the HistoryADS heuristic lead to a statistically significant reduction in the tree size, and those results marked with a "** represent cases where the unbound list did better with $95 \%$ confidence.

Consider Table 6.3, which presents our results for Focus with varying limits on the ADS' size. We observe the same pattern as we did in Othello and Checkers, in both the two-player and the multi-player cases, although the difference in savings between an unbounded list and a list of length 20 or 5 is quite a bit smaller, with even the worst case being $78 \%$ to $75 \%$ in the 4-ply midgame case, which was only a $3 \%$ difference. While the difference in savings between varying maximum lengths was not as pronounced as in Othello or Relaxed Checkers, we observe that it was still statistically significant in some cases, marked with a "*", at the initial board state. 
Table 6.1: Results of applying the History-ADS heuristic to Othello with a varying maximum length on the ADS.

\begin{tabular}{|c|c|c|c|c|c|c|c|}
\hline Ply & Mid & Limit & Avg. NC & Imp. & Std. Dev & P-Value & Effect \\
\hline 4 & No & No ADS & 669 & - & 205 & - & - \\
\hline 4 & No & Unbound & 525 & $21 \%$ & 175 & $3.2 \times 10^{-4}$ & 0.70 \\
\hline 4 & No & 20 & 572 & $15 \%$ & 196 & $5.8 \times 10^{-3}$ & 0.47 \\
\hline 4 & No & 5* & 596 & $11 \%$ & 196 & 0.041 & 0.36 \\
\hline 6 & $\mathrm{No}$ & No ADS & 5061 & - & 2385 & - & - \\
\hline 6 & No & Unbound & 3727 & $26 \%$ & 1552 & $1.7 \times 10^{-3}$ & 0.56 \\
\hline 6 & No & 20 & 3779 & $25 \%$ & 1884 & $3.3 \times 10^{-3}$ & 0.54 \\
\hline 6 & No & 5 & 3961 & $22 \%$ & 1603 & 0.013 & 0.46 \\
\hline 8 & No & No ADS & 38,800 & - & 20,300 & - & - \\
\hline 8 & No & Unbound & 23,600 & $40 \%$ & 10,800 & $<1.0 \times 10^{-5}$ & 0.75 \\
\hline 8 & No & 20 & 24,900 & $36 \%$ & 11,000 & $1.1 \times 10^{-5}$ & 0.68 \\
\hline 8 & No & $5^{*}$ & 28,600 & $26 \%$ & 12,200 & $5.8 \times 10^{-3}$ & 0.50 \\
\hline 4 & Yes & No ADS & 2199 & - & 745 & - & - \\
\hline 4 & Yes & Unbound & 1702 & $23 \%$ & 570 & $2.7 \times 10^{-4}$ & 0.67 \\
\hline 4 & Yes & 20 & 1787 & $19 \%$ & 663 & $2.6 \times 10^{-3}$ & 0.55 \\
\hline 4 & Yes & 5 & 1840 & $16 \%$ & 576 & 0.010 & 0.48 \\
\hline 6 & Yes & No ADS & 20,100 & - & 9899 & - & - \\
\hline 6 & Yes & Unbound & 13,300 & $34 \%$ & 6916 & $7.0 \times 10^{-5}$ & 0.69 \\
\hline 6 & Yes & 20 & 13,900 & $31 \%$ & 6846 & $6.9 \times 10^{-4}$ & 0.63 \\
\hline 6 & Yes & 5 & 14,800 & $26 \%$ & 7916 & $1.7 \times 10^{-3}$ & 0.54 \\
\hline 8 & Yes & No ADS & 182,500 & - & 114,000 & - & - \\
\hline 8 & Yes & Unbound & 92,900 & $49 \%$ & 49,700 & $<1.0 \times 10^{-5}$ & 0.79 \\
\hline 8 & Yes & 20 & 108,800 & $40 \%$ & 63,800 & $7.0 \times 10^{-5}$ & 0.65 \\
\hline 8 & Yes & $5^{*}$ & 115,900 & $36 \%$ & 59,800 & $3.1 \times 10^{-5}$ & 0.58 \\
\hline
\end{tabular}


Table 6.2: Results of applying the History-ADS heuristic to Relaxed Checkers with a varying maximum length on the ADS.

\begin{tabular}{|c||c||c||c||c||c||c||c|}
\hline \hline Ply & Mid & Limit & Avg. NC & Imp. & Std. Dev. & P-Value & Effect \\
\hline 4 & No & No ADS & 5930 & - & 864 & - & - \\
4 & No & Unbound & 4638 & $22 \%$ & 493 & $>1.0 \times 10^{-5}$ & 1.49 \\
4 & No & 20 & 4755 & $20 \%$ & 406 & $>1.0 \times 10^{-5}$ & 1.36 \\
4 & No & 5 & 4776 & $19 \%$ & 516 & $>1.0 \times 10^{-5}$ & 1.33 \\
\hline 6 & No & No ADS & 78,600 & - & 10,600 & - & - \\
6 & No & Unbound & 41,000 & $48 \%$ & 5588 & $<1.0 \times 10^{-5}$ & 3.55 \\
6 & No & 20 & 42,700 & $46 \%$ & 5292 & $<1.0 \times 10^{-5}$ & 3.39 \\
6 & No & $5^{*}$ & 44,700 & $44 \%$ & 5545 & $<1.0 \times 10^{-5}$ & 3.20 \\
\hline 8 & No & No ADS & 910,300 & - & 172,400 & - & - \\
8 & No & Unbound & 358,400 & $61 \%$ & 53,200 & $<1.0 \times 10^{-5}$ & 3.20 \\
8 & No & 20 & 374,800 & $59 \%$ & 55,700 & $<1.0 \times 10^{-5}$ & 3.11 \\
8 & No & $5^{*}$ & 397,600 & $56 \%$ & 78,300 & $<1.0 \times 10^{-5}$ & 2.97 \\
\hline \hline 4 & Yes & No ADS & 5447 & - & 1859 & - & - \\
4 & Yes & Unbound & 3681 & $33 \%$ & 1138 & $<1.0 \times 10^{-5}$ & 0.94 \\
4 & Yes & $20^{*}$ & 4130 & $24 \%$ & 1304 & $1.9 \times 10^{-4}$ & 0.70 \\
4 & Yes & $5^{*}$ & 4217 & $23 \%$ & 1363 & $4.3 \times 10^{-4}$ & 0.66 \\
\hline 6 & Yes & No ADS & 64,000 & - & 25,700 & - & - \\
6 & Yes & Unbound & 34,400 & $46 \%$ & 12,400 & $<1.0 \times 10^{-5}$ & 1.15 \\
6 & Yes & 20 & 36,700 & $43 \%$ & 14,200 & $<1.0 \times 10^{-5}$ & 1.06 \\
6 & Yes & 5 & 39,500 & $39 \%$ & 16,800 & $<1.0 \times 10^{-5}$ & 0.96 \\
\hline 8 & Yes & No ADS & 859,200 & - & 408,500 & - & - \\
8 & Yes & Unbound & 293,100 & $66 \%$ & 100,000 & $<1.0 \times 10^{-5}$ & 1.39 \\
8 & Yes & 20 & 332,900 & $61 \%$ & 132,700 & $<1.0 \times 10^{-5}$ & 1.29 \\
8 & Yes & $5^{*}$ & 397,100 & $54 \%$ & 192,500 & $<1.0 \times 10^{-5}$ & 1.13 \\
\hline
\end{tabular}


Table 6.3: Results of applying the History-ADS heuristic to Focus with a varying maximum length on the ADS.

\begin{tabular}{|c||c||c||c||c||c||c||c|}
\hline \hline Ply & Mid & Limit & Avg. NC & Imp. & Std. Dev. & P-Value & Effect \\
\hline 4 & No & No ADS & $5,254,700$ & - & 381,400 & - & - \\
4 & No & Unbound & $1,264,200$ & $76 \%$ & 90,900 & $<1.0 \times 10^{-5}$ & 10.46 \\
4 & No & 20 & $1,274,500$ & $76 \%$ & 96,300 & $<1.0 \times 10^{-5}$ & 10.43 \\
4 & No & $5^{*}$ & $1,332,200$ & $75 \%$ & 108,500 & $<1.0 \times 10^{-5}$ & 10.28 \\
\hline 4 (Multi) & No & No ADS & $6,973,500$ & - & 981,000 & - & - \\
4 (Multi) & No & Unbound & $2,150,300$ & $70 \%$ & 164,900 & $<1.0 \times 10^{-5}$ & 4.92 \\
4 (Multi) & No & 20 & $2,209,500$ & $69 \%$ & 164,500 & $<1.0 \times 10^{-5}$ & 4.86 \\
4 (Multi) & No & 5 & $2,275,200$ & $68 \%$ & 154,400 & $<1.0 \times 10^{-5}$ & 4.79 \\
\hline \hline 4 & Yes & No ADS & $10,602,200$ & - & $3,456,600$ & - & - \\
4 & Yes & Unbound & $2,388,700$ & $78 \%$ & 631,400 & $<1.0 \times 10^{-5}$ & 2.37 \\
4 & Yes & $20^{*}$ & $2,516,600$ & $76 \%$ & 709,800 & $<1.0 \times 10^{-5}$ & 2.34 \\
4 & Yes & $5^{*}$ & $2,628,500$ & $75 \%$ & 847,200 & $<1.0 \times 10^{-5}$ & 2.30 \\
\hline 4 (Multi) & Yes & No ADS & $14,192,200$ & - & $8,400,200$ & - & - \\
4 (Multi) & Yes & Unbound & $3,156,400$ & $79 \%$ & $1,695,300$ & $<1.0 \times 10^{-5}$ & 1.31 \\
4 (Multi) & Yes & 20 & $3,310,600$ & $77 \%$ & $1,697,300$ & $<1.0 \times 10^{-5}$ & 1.30 \\
4 (Multi) & Yes & 5 & $3,370,500$ & $76 \%$ & $1,406,800$ & $<1.0 \times 10^{-5}$ & 1.29 \\
\hline
\end{tabular}

Table 6.4 contains our results for the Virus Game. Our observations were analogous to those for the other three cases presented so far. While the History-ADS heuristic produced noticeable gains in all cases, they were lessened by a limit being placed on the maximum length of the ADS, or in many cases, mostly unchanged. Again, the worst change observed was a reduction in savings from $56 \%$ to $51 \%$ in the 6-ply case, or 4,649, 000 to 5,109,400, with measurements taken from the initial board state, which was, again, a very small change of only $5 \%$. While we observed that a limit of 20 caused very little decay in performance, a limit of 5 generally caused a statistically significant drop, although savings were still quite high. 
Table 6.4: Results of applying the History-ADS heuristic to the Virus Game with a varying maximum length on the ADS.

\begin{tabular}{|c||c||c||c||c||c||c||c|}
\hline \hline Ply & Mid & Limit & Avg. NC & Imp. & Std. Dev. & P-Value & Effect \\
\hline 4 & No & No ADS & 253,600 & - & 28,600 & - & - \\
4 & No & Unbound & 154,400 & $39 \%$ & 20,800 & $<1.0 \times 10^{-5}$ & 3.47 \\
4 & No & 20 & 157,500 & $38 \%$ & 19,000 & $<1.0 \times 10^{-5}$ & 3.36 \\
4 & No & $5^{*}$ & 163,200 & $36 \%$ & 19,000 & $<1.0 \times 10^{-5}$ & 3.20 \\
\hline 6 & No & No ADS & $10,463,700$ & - & $1,261,700$ & - & - \\
6 & No & Unbound & $4,649,000$ & $56 \%$ & 767,100 & $<1.0 \times 10^{-5}$ & 4.60 \\
6 & No & 20 & $4,832,300$ & $54 \%$ & 640,400 & $<1.0 \times 10^{-5}$ & 4.46 \\
6 & No & $5^{*}$ & $5,109,400$ & $51 \%$ & 657,800 & $<1.0 \times 10^{-5}$ & 4.24 \\
\hline \hline 4 & Yes & No ADS & 308,800 & - & 40,700 & - & - \\
4 & Yes & Unbound & 186,800 & $39 \%$ & 23,100 & $<1.0 \times 10^{-5}$ & 3.00 \\
4 & Yes & 20 & 186,500 & $39 \%$ & 22,200 & $<1.0 \times 10^{-5}$ & 3.00 \\
4 & Yes & 5 & 194,300 & $37 \%$ & 21,600 & $<1.0 \times 10^{-5}$ & 2.81 \\
\hline 6 & Yes & No ADS & $12,801,300$ & - & $1,952,500$ & - & - \\
6 & Yes & Unbound & $5,872,700$ & $55 \%$ & 862,900 & $<1.0 \times 10^{-5}$ & 3.55 \\
6 & Yes & 20 & $5,913,100$ & $54 \%$ & 821,300 & $<1.0 \times 10^{-5}$ & 3.53 \\
6 & Yes & $5^{*}$ & $6,397,000$ & $51 \%$ & 746,900 & $<1.0 \times 10^{-5}$ & 3.29 \\
\hline
\end{tabular}

Finally, Table 6.5 presents our results for Chinese Checkers. The patterns we observed were similar to those for the other four games. However, in the case of 4 player Chinese Checkers, from the initial board position, a maximum list size of 5 outperformed a list size of 20 . In general, for this game, the variability was very small, such as from $62 \%$ to $61 \%$ in the 4-ply, six player case, with a limit on the list of size of 20 , from $1,282,500$ to $1,331,900$. As before, even considering the worst situation, the largest change observed was from $66 \%$ to $62 \%$, in the 4-ply, four player case, with measurements taken from the initial board position. In this case, although a more restricted list usually performed marginally worse, none of the limits on length were significantly worse than each other. 
Table 6.5: Results of applying the History-ADS heuristic to Chinese Checkers with a varying maximum length on the ADS.

\begin{tabular}{|c||c||c||c||c||c||c||c|}
\hline \hline Ply & Mid & Limit & Avg. NC & Imp. & Std. Dev. & P-Value & Effect \\
\hline 4 (4-play) & No & No ADS & $1,376,800$ & - & 416,800 & - & - \\
4 (4-play) & No & Unbound & 475,600 & $66 \%$ & 124,700 & $<1.0 \times 10^{-5}$ & 2.16 \\
4 (4-play) & No & 20 & 530,700 & $62 \%$ & 125,200 & $<1.0 \times 10^{-5}$ & 2.03 \\
4 (4-play) & No & 5 & 525,000 & $62 \%$ & 156,600 & $<1.0 \times 10^{-5}$ & 2.04 \\
\hline 4 (6-play) & No & No ADS & $3,366,300$ & - & $1,097,500$ & - & - \\
4 (6-play) & No & Unbound & $1,282,500$ & $62 \%$ & 368,000 & $<1.0 \times 10^{-5}$ & 1.90 \\
4 (6-play) & No & 20 & $1,331,900$ & $61 \%$ & 331,800 & $<1.0 \times 10^{-5}$ & 1.85 \\
4 (6-play) & No & 5 & $1,361,200$ & $60 \%$ & 297,500 & $<1.0 \times 10^{-5}$ & 1.83 \\
\hline \hline 4 (4-play) & Yes & No ADS & $3,340,200$ & - & 933,000 & - & - \\
4 (4-play) & Yes & Unbound & $1,236,700$ & $63 \%$ & 335,000 & $<1.0 \times 10^{-5}$ & 2.25 \\
4 (4-play) & Yes & 20 & $1,328,900$ & $60 \%$ & 329,900 & $<1.0 \times 10^{-5}$ & 2.16 \\
4 (4-play) & Yes & 5 & $1,367,700$ & $59 \%$ & 454,700 & $<1.0 \times 10^{-5}$ & 2.11 \\
\hline 4 (6-play) & Yes & No ADS & $8,259,600$ & - & $2,640,800$ & - & - \\
4 (6-play) & Yes & Unbound & $3,200,100$ & $61 \%$ & 817,900 & $<1.0 \times 10^{-5}$ & 1.92 \\
4 (6-play) & Yes & 20 & $3,294,200$ & $60 \%$ & 840,800 & $<1.0 \times 10^{-5}$ & 1.88 \\
4 (6-play) & Yes & 5 & $3,341,800$ & $60 \%$ & 932,600 & $<1.0 \times 10^{-5}$ & 1.86 \\
\hline
\end{tabular}

The above results support our hypothesis that the History-ADS heuristic is capable of maintaining most of its performance even when the length of its list is restricted. A more detailed discussion will be presented in Section 6.5. 


\subsection{Multi-level ADSs}

This section describes our investigation in using multiple ADSs, one for each level of the tree.

\subsubsection{Strategy and Technique}

The History-ADS heuristic maintains a single adaptive list, which is updated whenever a move produces a cut, and is used to order moves when they are encountered elsewhere in the tree. It performs this operation "blindly", without giving consideration to the location in the tree where the move produced a cut, relative to its current location. Thus, if moves are found to produce cuts at the lowest levels of the tree, they will be prioritized at the upper levels of the tree later in the search.

While this may lead to improved savings, as certain moves may be very strong regardless of which level of the tree they occur on, there is a potential weakness in such a blind invocation. Consider the case where a move produces a cut at the highest level of the tree, at node $N$. It is thus added to the adaptive list, and the search continues deeper into the tree, exploring it in a depth-first manner. Deeper in the tree, many moves are likely to produce cuts, and these will be added to the adaptive list ahead of the first move. When the search returns to the higher levels of the tree, and explores a neighbour of $N$, these moves will be prioritized first, over the move that produced a cut at its neighbour. However, intuitively the move that produced a cut at $N$, which is a more similar game state compared to those deeper in the tree, is likely to be stronger at the current node.

Furthermore, by handling all moves equally, as there are many more nodes towards the bottom of the tree compared to the top, moves that are strong near the bottom of the tree will receive many more updates and thus a higher ranking in the adaptive list. This will occur even though cuts near the top of the tree are comparatively more valuable. Both the Killer Moves and History heuristics employ mechanisms to 
mitigate these effects [54]. Inspired by this, we augment the History-ADS heuristic with multiple ADSs, one for each level, and use them only within the contexts of their sibling nodes. An example of the History-ADS using multiple ADSs, one for each level, is provided in Figure 6.2.
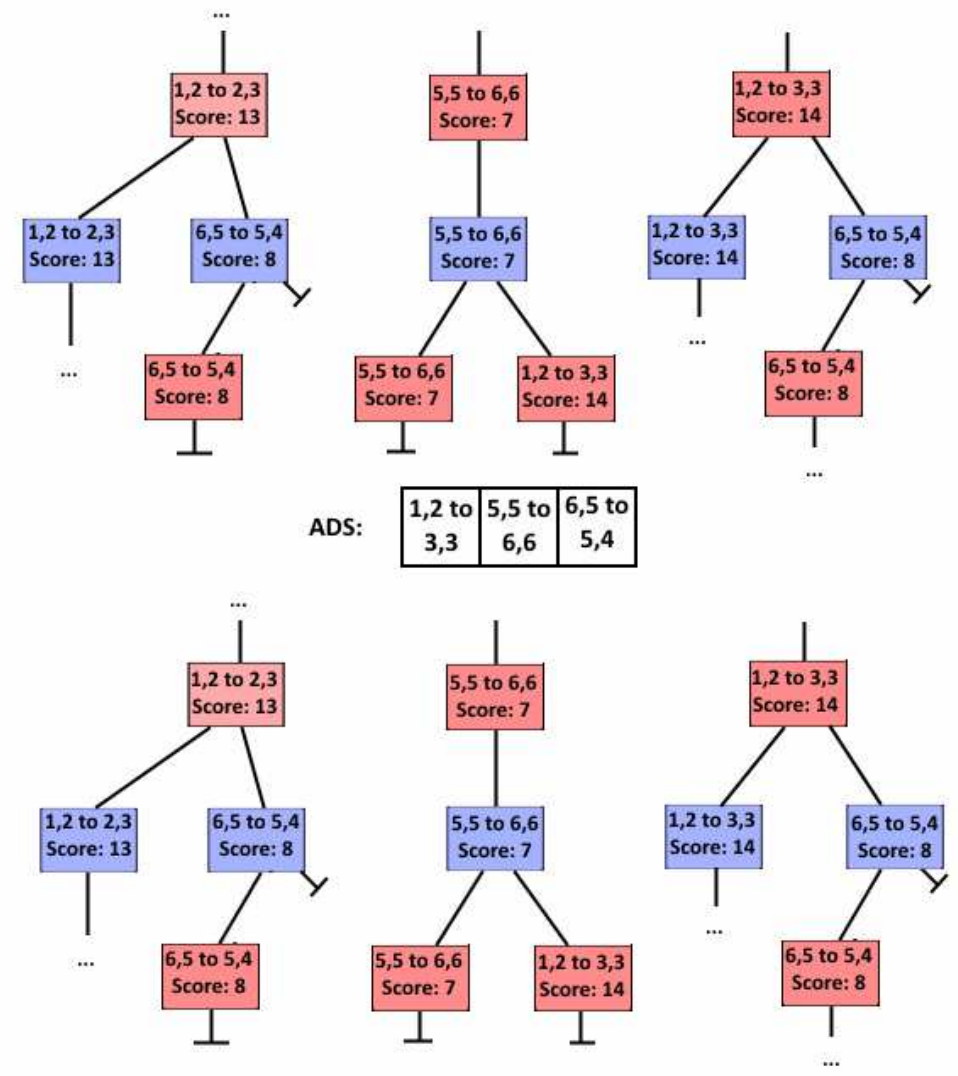

ADSs
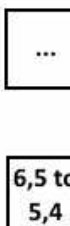

Figure 6.2: An example of the utility of multiple ADSs in the context of the HistoryADS heuristic. In the figure on the top, the incorrect move is prioritized due to it producing a cut lower in the tree in a completely different region of the search.

The use of multi-level ADSs may lead to a reduction in performance, given that learning cannot be applied at different levels of the tree. But given the precedence set by the existing techniques reported in the literature and the potential benefits, we consider it a meaningful avenue of inquiry. 


\subsubsection{Experimental Model}

The suite of experiments conducted for multi-level ADSs are largely analogous to those described earlier, for our analysis into bounded lists. We employ the same software system, and based on our earlier results, invoke only the Move-to-Front rule as our update mechanism. As before, we record the aggregate NC over the same number of turns, from both midgame and initial board positions.

The trials are also configured identically to those for bounded lists, with the History-ADS heuristic utilizing a multi-level ADS. We also consider multi-level ADSs with a length limit of 5 , with the goal of jointly combining the two phenomena presented in this chapter. We choose the length limit of 5 because, as we are already maintaining multiple ADSs, we can hold more information than a single ADS with a length that is limited to 5 .

Compared to the case of examining a maximum length on the ADS, we only perform experiments involving multi-level ADSs in cases where the ply depth is at least 6 . The reason for this is that as it stands, the History-ADS heuristic maintains separate ADSs for each player, and pruning cannot take place at the top level (the root) or the bottom (the leaf nodes), implying that the multi-level ADS approach is identical to a single ADS for 4-ply trials.

The same Intel i5 $3.4 \mathrm{GHz}$ processor was employed as in the previous section. We again use the Mann-Whitney test to determine the statistical significance, and provide the Effect Size metric.

\subsubsection{Experimental Results for Multi-Level ADSs}

Table 6.6 presents our results for multi-level ADSs, in the domain of Othello. The values for a non-multi-level ADS are the same as those for an unbounded ADS in the previous section. We observed that, similar to the limit on the ADS, the use of a multi-level ADS reduces performance by a consistent but small amount. Limiting the 
multi-level ADS's size further reduces the performance, in the majority of cases. In the best case, savings were reduced from $39 \%$ to $36 \%$, from 23,600 to 24,900 , when a multi-level ADS with no size limitation was employed, in the 8-ply case, from the initial board position. In all cases but one, the History-ADS produced statistically significant improvements in tree pruning, the exception being the use of multi-level ADSs with a length bounded by 5 , in the 6-ply case from the initial board state. However, in only a single case, marked with a "*", was the difference between multilevel ADSs and non-multi-level ADSs itself significant to a $95 \%$ certainty threshold.

Table 6.6: Results of applying the History-ADS heuristic to Othello with multi-level ADSs.

\begin{tabular}{|c||c||c||c||c||c||c||c|}
\hline \hline Ply & Mid & Multi? & Avg. NC & Imp. & Std. Dev. & P-Value & Effect \\
\hline 6 & No & No ADS & 5061 & - & 2385 & - & - \\
6 & No & No & 3727 & $26 \%$ & 1552 & $1.7 \times 10^{-3}$ & 0.56 \\
6 & No & Yes & 4110 & $19 \%$ & 1828 & 0.023 & 0.40 \\
6 & No & Yes (5-limit) & 4305 & $15 \%$ & 1843 & 0.089 & 0.37 \\
\hline 8 & No & No ADS & 38,800 & - & 20,300 & - & - \\
8 & No & No & 23,600 & $39 \%$ & 10,800 & $<1.0 \times 10^{-5}$ & 0.75 \\
8 & No & Yes & 24,900 & $36 \%$ & 11,900 & $1.0 \times 10^{-4}$ & 0.68 \\
8 & No & Yes (5-limit) & 25,600 & $34 \%$ & 13,100 & $2.9 \times 10^{-4}$ & 0.65 \\
\hline \hline 6 & Yes & No ADS & 20,100 & - & 9899 & - & - \\
6 & Yes & No & 13,300 & $34 \%$ & 6916 & $7.0 \times 10^{-5}$ & 0.69 \\
6 & Yes & Yes & 14,700 & $27 \%$ & 7279 & $1.8 \times 10^{-3}$ & 0.55 \\
6 & Yes & Yes (5-limit) & 16,000 & $20 \%$ & 7283 & 0.017 & 0.42 \\
\hline 8 & Yes & No ADS & 182,500 & - & 114,000 & - & - \\
8 & Yes & No & 92,900 & $49 \%$ & 49,700 & $<1.0 \times 10^{-5}$ & 0.79 \\
8 & Yes & Yes & 109,600 & $40 \%$ & 56,500 & $1.0 \times 10^{-4}$ & 0.64 \\
8 & Yes & Yes (5-limit)* & 104,500 & $42 \%$ & 50,600 & $<1.0 \times 10^{-5}$ & 0.68 \\
\hline
\end{tabular}


Our results for Relaxed Checkers are shown in Table 6.7. The patterns observed were similar to those for Othello, with the use of multi-level ADSs performing slightly worse than the original version, although noticeably worse than in the case of Othello, with cases where the difference between the single-level and multi-level ADS was itself significant noted with a "*", as usual. The smallest change was from $46 \%$ to $40 \%$, in the 6-ply case, where measurements were taken from a midgame board position.

Table 6.7: Results of applying the History-ADS heuristic to Relaxed Checkers with multi-level ADSs.

\begin{tabular}{|c||c||c||c||c||c||c||c|}
\hline \hline Ply & Mid & Multi? & Avg. NC & Imp. & Std. Dev. & P-Value & Effect \\
\hline 6 & No & No ADS & 78,600 & - & 10,600 & - & - \\
6 & No & No & 41,000 & $48 \%$ & 5588 & $<1.0 \times 10^{-5}$ & 3.55 \\
6 & No & Yes* & 45,300 & $42 \%$ & 5977 & $<1.0 \times 10^{-5}$ & 3.14 \\
6 & No & Yes (5-limit)* & 46,900 & $40 \%$ & 6727 & $<1.0 \times 10^{-5}$ & 3.00 \\
\hline 8 & No & No ADS & 910,300 & - & 172,400 & - & - \\
8 & No & No & 358,400 & $61 \%$ & 53,200 & $<1.0 \times 10^{-5}$ & 3.20 \\
8 & No & Yes & 394,300 & $57 \%$ & 53,500 & $<1.0 \times 10^{-5}$ & 2.99 \\
8 & No & Yes (5-limit) & 416,400 & $54 \%$ & 68,300 & $<1.0 \times 10^{-5}$ & 2.86 \\
\hline \hline 6 & Yes & No ADS & 64,000 & - & 25,700 & - & - \\
6 & Yes & No & 34,400 & $46 \%$ & 12,400 & $>1.0 \times 10^{-5}$ & 1.15 \\
6 & Yes & Yes* & 38,100 & $40 \%$ & 15,400 & $>1.0 \times 10^{-5}$ & 1.06 \\
6 & Yes & Yes (5-limit)* & 37,800 & $41 \%$ & 13,000 & $>1.0 \times 10^{-5}$ & 1.02 \\
\hline 8 & Yes & No ADS & 859,200 & - & 408,500 & - & - \\
8 & Yes & No & 293,100 & $66 \%$ & 100,000 & $>1.0 \times 10^{-5}$ & 1.39 \\
8 & Yes & Yes & 350,400 & $59 \%$ & 162,900 & $>1.0 \times 10^{-5}$ & 1.25 \\
8 & Yes & Yes (5-limit)* & 403,900 & $53 \%$ & 149,700 & $>1.0 \times 10^{-5}$ & 1.11 \\
\hline
\end{tabular}

Lastly, we present our results for the Virus Game with multi-level ADSs in Table 6.8. While we observed, as before, that the performance worsened when the multilevel approach was employed, the difference was quite a bit smaller in the context of the Virus Game. The loss in savings was very slight in the best case, from $56 \%$ to 
$55 \%$, or from 4,649,000 to 4,744,400, from the initial board position. In the case of the Virus Game, the difference between the multi-level ADS approach and the standard History-ADS heuristic was not enough to achieve statistical significance.

Table 6.8: Results of applying the History-ADS heuristic to the Virus Game with multi-level ADSs.

\begin{tabular}{|c||c||c||c||c||c||c||c|}
\hline \hline Ply & Mid & Multi? & Avg. NC & Imp. & Std. Dev. & P-Value & Effect \\
\hline 6 & No & No ADS & $10,463,700$ & - & $1,261,700$ & - & - \\
6 & No & No & $4,649,000$ & $56 \%$ & 767,100 & $>1.0 \times 10^{-5}$ & 4.60 \\
6 & No & Yes & $4,744,400$ & $55 \%$ & 597,700 & $>1.0 \times 10^{-5}$ & 4.53 \\
6 & No & Yes (5-limit) & $4,800,200$ & $54 \%$ & 817,200 & $>1.0 \times 10^{-5}$ & 4.49 \\
\hline \hline 6 & Yes & No ADS & $12,801,200$ & - & $1,952,500$ & - & - \\
6 & Yes & No & $5,872,700$ & $55 \%$ & 862,900 & $>1.0 \times 10^{-5}$ & 3.55 \\
6 & Yes & Yes & $5,989,700$ & $53 \%$ & 652,600 & $>1.0 \times 10^{-5}$ & 3.49 \\
6 & Yes & Yes (5-limit) & $6,159,300$ & $52 \%$ & 833,200 & $>1.0 \times 10^{-5}$ & 3.40 \\
\hline
\end{tabular}

\subsection{Discussion}

Our results in this chapter support those presented earlier for the History-ADS heuristic, demonstrating that the History-ADS heuristic is able to consistently produce large improvements in tree pruning, over a wide range of games and configurations.

We found that when limiting the maximum size of the ADS, while there was some reduction in performance, as was expected, the loss was very slight in most cases. This confirms our hypothesis that elements near the head of the list tend to remain there, and provide the majority of the move ordering benefits, with diminishing returns as the list gets longer. The fact that the majority of savings are still maintained in all cases even when the list is limited to only have five elements, successfully addresses one of the concerns we had with of the History-ADS heuristic, namely that the length of the adaptive list can potentially be quite large. 
The trend was consistent across all cases, with the limit of 20 performing marginally worse than an unbound list, and the limit of 5 doing a little worse. The performance varied between game models, however in the best case, tied with a number of others, saw a reduction of only $1 \%$, from $62 \%$ to $61 \%$, in six-player Chinese Checkers. Even in the worse cases, we observed that the majority of the savings remained, even when the list is bounded by a maximum of 5 . While this pattern was consistent across all cases, it was only statistically significant in approximately half the cases, spread roughly evenly amongst the games. The exception to this is Chinese Checkers, which, similar to the last chapter, deviated from the pattern in that it was much more uniform. This further reinforces our suggestion that in the case of Chinese Checkers, only a few similar moves are necessary to achieve the majority of the pruning available to the History-ADS heuristic.

The multi-level ADS approach was found to do worse than the single ADS approach, suggesting that savings that may be gained for prioritizing moves that produced a cut on the current level of the tree, if they exist, are offset by the inability to apply what the algorithm has learned to other levels of the tree. We can thus conclude that the "absolutist" approach of having a separate ADS at each level of the tree is likely not the optimal way to address concerns of overvaluing moves that are strong near the bottom of the tree. However, the multi-level ADS approach may not be completely useless. If the History-ADS heuristic is employed alongside other, perhaps domain-specific move ordering heuristics, then prioritizing only the best moves at each level may be as effective. This approach begs for more investigation.

Despite the presence of multiple ADSs, limiting the size of the list to 5 reduced improvements even more. Observing the internal functioning of the search, the reason for this appears to be that the limit is only a factor at the lowest levels of the tree, where many more moves produce cuts and the limit impacts the ADS heavily. As opposed to this, levels closer to the root do not require as much space. This is especially visible in the case of 8-ply Othello from the midgame case, where a multilevel ADS with a maximum size of 5 did not, in fact, produce a statistically significant improvement in tree pruning, which is the first time that such an event has occurred 
for the History-ADS heuristic. Overall, however, the multi-level ADS' performance was close to the original version in most cases.

A final point of interest is that, combining the concepts of multi-level ADSs and bounded lists, the existing Killer Moves heuristic can, in fact, be modeled in terms of the History-ADS heuristic. This can be accomplished with a multi-level ADS with a bounded length of 2, as, summarizing the discussion from Chapter 2, traditionally two Killer Moves are maintained for each level of the tree, employing the Move-toFront heuristic. The Move-to-Front heuristic accomplishes the replacement scheme of keeping the most recent Killer Move, and the oldest one. Different replacement schemes can be accomplished through different update mechanisms, and maintaining more Killer Moves per level can be achieved by changing the bound on the list.

Relying on our observations on the multi-level ADS approach, in the next chapter we will build on this further to allow learning from other levels of the tree to be used, while still attempting to manage issues of nodes near the bottom of the tree being overly favoured.

\subsection{Chapter Conclusions}

Our work in this chapter has examined the effects of two possible refinements of the History-ADS heuristic. The first of these was a limitation on the length of the list, to bound and reduce the memory footprint of the History-ADS heuristic. The second involved providing a separate ADS for each level of the game tree. Our results provide strong evidence that while some loss of performance is observed by restricting the ADS' length, the majority of savings remain even with a very short list. The multilevel approach also led to some loss of performance, suggesting that the application of learning at multiple levels of the tree is more important than prioritizing nodes that produce cuts at sibling nodes. 
A further attempt to manage prioritization of moves from multiple levels of the ADS, and an examination of the History-ADS heuristic in comparison to established move ordering strategies, is the focus of the next chapter. 


\section{Chapter 7}

\section{History-ADS Compared to Existing Move-Ordering Heuristics}

\subsection{Chapter Overview}

In the previous chapters, we introduced the concept of ordering moves based on their history by employing the mechanisms of an ADS, leading to the creation of the History-ADS heuristic. The History-ADS heuristic demonstrated that it could achieve substantial reductions in tree size in a wide variety of two-player and multiplayer cases. In the last chapter, we also examined two possible refinements to the History-ADS heuristic, inspired by both the History Heuristic and the Killer Moves heuristic, both of which are well-known move ordering techniques, which also employ a history-like metric.

Given the strong performance of the History-ADS heuristic, and its inspiration from these two highly-regarded strategies, in this chapter we will directly compare the History-ADS with both the History Heuristic, and Killer Moves. We will also present an alternative, approximate implementation of the History Heuristic using another ADS. Given our previous observations, we expect that the History-ADS heuristic will 
perform on a level that is comparable with these established techniques, and we have reason to strongly suspect it may, in fact, do better in many environments ${ }^{1}$.

\subsection{Motivation}

We have earlier demonstrated the potential benefits of the History-ADS heuristic in a wide range of environments, and explored a large number of possible configurations within these environments. Unlike the Threat-ADS heuristic, however, which pioneered an entirely new concept, i.e., that of using opponent threats to achieve move ordering, the History-ADS heuristic achieves move ordering through move history, a known metric. While large reductions, of up to $78 \%$, for a relatively lightweight technique, clearly demonstrate the success of the History-ADS, we cannot be sure of its actual relative benefits, unless it is compared to the established, well-known techniques of a similar nature.

Given the similarities in principle behind the History-ADS heuristic, and both the History Heuristic and Killer Moves, we have elected to present a comparison between its performance and these two well-known methods, under a testing domain that is similar to the one employed in the previous chapters. By providing this comparison, we believe that we will be able to place the History-ADS heuristic's achievements in the proper context and perspective. Furthermore, it may actually be the case that the History-ADS heuristic is able to outperform one or both of them, which would be a very valuable result, worthy of recognition.

In fact, we have reason to suspect that the History-ADS heuristic may be capable of outperforming the History Heuristic in at least some domains. This is based on the premise of our results presented in Chapter 5, and more specifically, on the fact that the Move-to-Front rule almost always performed better than the Transposition rule. The History Heuristic, described in Chapter 2, employs a more complex mechanism

\footnotetext{
${ }^{1}$ The work in this chapter was originally published in [42]
} 
to rank moves. It is, however, less sensitive to change than the Move-to-Front rule. It may prove to be the case that, in at least some domains, the extreme adaptability of the Move-to-Front rule will outperform even the elaborate History Heuristic. This begs investigation.

The Killer Moves heuristic is already conceptually very similar to the multi-level History-ADS heuristic. In fact, it can be implemented in terms of the History-ADS, as will be explained presently. Again, given that Killer Moves retains fewer moves per level of the tree than any variant of the multi-level History-ADS explored in the previous chapter, it is likely that this trend will continue, and that the History-ADS will be able to outperform it in a range of domains.

The potential to achieving performance on the level of the well-regarded History Heuristic and Killer Moves, using an ADS, motivates the work presented in this chapter.

\subsection{ADS-based implementation of the History Heuris- tic}

We have already observed that a potential drawback of the History-ADS heuristic is based on its application of the ADS equivalently at all levels of the tree. This is because it maintains a single adaptive list of moves, which is updated according to its update mechanism whenever a cut is produced, regardless of the level of the tree in which that cut originated. This information is then used to order moves in all levels of the tree, which presents a potential "infirmity", as a move that was found to be strong on a specific level of the tree, may not be effective at a different ply depth. Indeed, this is a factor that both the History Heuristic and Killer Moves compensate for [54]. Furthermore, moves that produce cuts near the bottom of the tree would have a disproportionate impact on its structure. 
Based on this reasoning, a potential refinement of the History-ADS heuristic occurs when a separate ADS is maintained for each level of the tree, as referred to by the concept of "multi-level ADSs". However, while this configuration continued to produce strong move ordering in nearly all examined cases, it was generally outperformed by the basic version of the History-ADS. We reasoned that this was likely due to the scheme's inability to apply the learning achieved at other levels of the game tree at all ply levels. Consequently, while some gains may have been made by avoiding prioritizing incorrect moves, they did not outpace losses due to this effect.

It should be emphasized that the History Heuristic uses a single data structure, applied at all levels of the tree, and mitigates the impact of strong moves from deeper ply depths by maintaining a set of counters associated with each possible move. These counters are incremented by a much greater amount if the move produces a cut higher in the tree (as described in Chapter 2). Inspired by this, in this chapter we present an ADS implementation of this strategy, which modifies the History-ADS heuristic to employ the concept of such a "variable" counter.

The History-ADS heuristic does not maintain counters associated with the moves within the ADS. This is a feature that is intentionally included in the design, because the use of counters would necessitate sorting, and would require the History-ADS to employ the Frequency Count approach, which is well-known for its weaknesses. However, the acclaimed Move-Ahead- $k$ update mechanism, discussed earlier in this work and tested with the Threat-ADS heuristic, provides a mechanism by which we can utilize the History Heuristic's counter updates, within the context of the HistoryADS heuristic.

With regard to the Move-Ahead- $k$ rule, rather than set $k$ to be a fixed value, we will modify it contextually, based on the location in the game tree at which a cut occurred. The intention is to use this mechanism to mimic how the History Heuristic increments its counters. Specifically, when a cut occurs, we will set $k$ to be depth $\times$ depth (recall that depth has the value of unity at the bottom of the tree, and has a larger value higher up), analogous to the History Heuristic's original specification [54]. Thus, cuts 
at higher levels will cause moves to advance much farther forward in the list than those closer to the bottom. An example of the execution of this strategy is presented in Figure 7.1.

\begin{tabular}{|c|c|c|c|c|c|}
\hline 7,8 to & 4,5 to & 3,2 to & 6,7 to & 1,3 to & 2,1 to \\
8,8 & 5,4 & 5,1 & 6,6 & 1,6 & 2,2 \\
\hline
\end{tabular}

\begin{tabular}{|c|c|c|c|c|c|c|}
\hline 7,8 to & 4,5 to & 1,1 to & 3,2 to & 6,7 to & 1,3 to & 2,1 to \\
$\mathbf{8 , 8}$ & 5,4 & 4,1 & 5,1 & 6,6 & 1,6 & $\mathbf{2 , 2}$ \\
\hline
\end{tabular}

\begin{tabular}{|c|c|c|c|c|c|c|}
\hline $\mathbf{7 , 8}$ to & 1,3 to & $\mathbf{4 , 5}$ to & $\mathbf{1 , 1}$ to & $\mathbf{3 , 2}$ to & $\mathbf{6 , 7}$ to & $\mathbf{2 , 1}$ to \\
$\mathbf{8 , 8}$ & 1,6 & $\mathbf{5 , 4}$ & $\mathbf{4 , 1}$ & $\mathbf{5 , 1}$ & $\mathbf{6 , 6}$ & $\mathbf{2 , 2}$ \\
\hline
\end{tabular}

\begin{tabular}{|c|c|c|c|c|c|c|}
\hline 1,1 to & $\mathbf{7 , 8}$ to & $\mathbf{1 , 3}$ to & $\mathbf{4 , 5}$ to & $\mathbf{3 , 2}$ to & $\mathbf{6 , 7}$ to & 2,1 to \\
4,1 & $\mathbf{8 , 8}$ & $\mathbf{1 , 6}$ & $\mathbf{5 , 4}$ & $\mathbf{5 , 1}$ & $\mathbf{6 , 6}$ & $\mathbf{2 , 2}$ \\
\hline
\end{tabular}

Figure 7.1: A demonstration of how our approximation of the History Heuristic using an ADS updates the list of available moves. The move $(1,1)$ to $(4,1)$ is found to produce a cut at depth 2 , and is added to the end of the list, and then moved ahead by a value of 4 (depth $\times$ depth) places. Thereafter, the move $(1,3)$ to $(1,6)$ is found to produce a cut, also at depth 2 , and is moved ahead by 4 places as well. Finally, $(1,1)$ to $(4,1)$ produces a cut at depth 3 , and is moved ahead 9 places, to the front.

We remark that this is not a perfect simulation of the History Heuristic. This is because the lack of counters can produce such a scenario, as depicted in Figure 7.1, where a move can only be placed at the head of the list, not beyond it. Thus, this ADS-based approximation of the History Heuristic will remain slightly more susceptible to cuts deeper in the tree having a disproportionate impact. However, the benefits of the History-ADS heuristic, which include a smaller data structure and which do not require sorting, are very likely to outpace these drawbacks. 


\subsection{Experimental Model}

Our experiments in this chapter are necessarily analogous to those presented previously in this work for the History-ADS heuristic, particularly to those from the previous chapter. We wish to examine the performance of our ADS-based implementation of the History Heuristic's update mechanism, which we will abbreviate as HH-ADS, in the same environments in which we examined the limitations on the length of the ADS, and in the initial trials for the History-ADS. To avoid repetition, we refer the reader to the previous chapters for the specific test cases that will be employed, and why each is chosen.

Aside from the HH-ADS implementation, we will also present, for the first time, a direct comparison between the History-ADS, and the History Heuristic itself. We employ an implementation of the original history heuristic as specified in its introductory paper, where moves are identified by their originating and destination squares on the game board (as the History-ADS heuristic operates), and each counter is incremented by the value of depth $\times$ depth when a cut is produced.

As the HH-ADS and the History Heuristic have specific implementations, based on the established literature, neither of them require any additional parameters. As before, all experiments are done using the same software system, running on the same Intel i5 3.4 GHz processor. We present the average $\mathrm{NC}$, and employ the MannWhitney test for statistical significance, and also, as before, provide the Effect Sizes. Our results are presented in the next section. 


\subsection{Results for the History Heuristic}

Our results comparing the History-ADS heuristic, HH-ADS, and the History Heuristic in the context of Othello are presented in Table 7.1. The reader can see that, although the performances were very similar in most situations, the History-ADS heuristic outperformed both the new HH-ADS, and the History Heuristic itself, in every case. For example, in the 6-ply case from an initial board position, the History-ADS heuristic produced an average NC of 3,727, and the History Heuristic 4, 136 (contrast with no ADS in the previous chapter having an average of 5,061). We observed that in every situation, the History-ADS and HH-ADS produced statistically significant change from the default (omitted from the tables in this chapter, but provided previously). As opposed to this, the History Heuristic did not achieve 95\% certainty in the case of 6-ply Othello, with measurements taken from the initial board state. The Effect Size measures were between 0.5 and 0.8 , indicating a moderate to large effect, with an expectedly lower measure for the History Heuristic.

Table 7.2 showcases our results comparing the three techniques in the context of Relaxed Checkers. We observed that, although the same pattern was observed as in the context of Othello, with the original History-ADS heuristic having outperformed HH-ADS, which in turn outperformed the History Heuristic, the History Heuristic itself did noticeably worse in some cases. This happened particularly where the number of nodes was larger, such as in the 8-ply case from the initial board state, where the History-ADS heuristic performed 37\% better, producing a NC of 358,400 compared to 567,900. In all cases, this time, all heuristics generated an improvement in tree pruning with a very high statistical certainty, and the Effect Sizes ranged between 0.8 and 3.0, indicating a very large effect.

Table 7.3 holds our results for Focus, and we saw a very similar pattern yet again, with the HH-ADS having been slightly outperformed by the History-ADS heuristic, and the original History Heuristic did noticeably worse in all cases, with the HistoryADS heuristic doing $38 \%$ better in the case of multi-player focus at the midgame state. In all cases, as in the previous two chapters, every heuristic produced a very 
Table 7.1: Results comparing the History-ADS, the HH-ADS, and the History Heuristic in Othello.

\begin{tabular}{|c||c||c||c||c||c||c|}
\hline \hline Ply & Mid & Method & Avg. NC & Std. Dev. & P-Value & Effect \\
\hline 4 & No & History-ADS & 525 & 175 & $3.2 \times 10^{-4}$ & 0.70 \\
4 & No & HH-ADS & 570 & 191 & 0.011 & 0.46 \\
4 & No & History Heuristic & 575 & 196 & 0.018 & 0.34 \\
\hline 6 & No & History-ADS & 3727 & 1552 & $1.7 \times 10^{-3}$ & 0.56 \\
6 & No & HH-ADS & 3789 & 1659 & $7.3 \times 10^{-3}$ & 0.53 \\
6 & No & History Heuristic & 4136 & 1711 & 0.071 & 0.37 \\
\hline 8 & No & History-ADS & 23,600 & 10,800 & $<1.0 \times 10^{-5}$ & 0.75 \\
8 & No & HH-ADS & 24,000 & 11,600 & $<1.0 \times 10^{-5}$ & 0.73 \\
8 & No & History Heuristic & 24,100 & 10,200 & $<1.0 \times 10^{-5}$ & 0.72 \\
\hline \hline 4 & Yes & History-ADS & 1701 & 570 & $2.7 \times 10^{-4}$ & 0.67 \\
4 & Yes & HH-ADS & 1731 & 623 & $6.2 \times 10^{-4}$ & 0.63 \\
4 & Yes & History Heuristic & 1779 & 628 & $5.5 \times 10^{-3}$ & 0.56 \\
\hline 6 & Yes & History-ADS & 13,300 & 6916 & $7.0 \times 10^{-5}$ & 0.69 \\
6 & Yes & HH-ADS & 13,400 & 5801 & $6.9 \times 10^{-5}$ & 0.68 \\
6 & Yes & History Heuristic & 15,500 & 6939 & $9.4 \times 10^{-3}$ & 0.47 \\
\hline 8 & Yes & History-ADS & 92,900 & 50,000 & $<1.0 \times 10^{-5}$ & 0.79 \\
8 & Yes & HH-ADS & 95,500 & 47,000 & $7.0 \times 10^{-5}$ & 0.76 \\
8 & Yes & History Heuristic & 111,100 & 57,400 & $1.5 \times 10^{-4}$ & 0.63 \\
\hline
\end{tabular}

statistically strong impact on the NC. Again, the Effect Sizes for the History Heuristic are slightly smaller, as would be expected given its smaller impact on tree pruning, with the Effect Sizes, in general, being very large, with the smallest being over 1.0. 
Table 7.2: Results comparing the History-ADS, the HH-ADS, and the History Heuristic in Relaxed Checkers.

\begin{tabular}{|c||c||c||c||c||c||c|}
\hline \hline Ply & Mid & Method & Avg. NC & Std. Dev. & P-Value & Effect \\
\hline 4 & No & History-ADS & 4649 & 493 & $<1.0 \times 10^{-5}$ & 1.49 \\
4 & No & HH-ADS & 4854 & 612 & $<1.0 \times 10^{-5}$ & 1.24 \\
4 & No & History Heuristic & 5041 & 609 & $<1.0 \times 10^{-5}$ & 1.03 \\
\hline 6 & No & History-ADS & 41,000 & 5588 & $<1.0 \times 10^{-5}$ & 3.55 \\
6 & No & HH-ADS & 46,800 & 4783 & $<1.0 \times 10^{-5}$ & 3.00 \\
6 & No & History Heuristic & 54,800 & 9018 & $<1.0 \times 10^{-5}$ & 2.25 \\
\hline 8 & No & History-ADS & 358,400 & 53,200 & $<1.0 \times 10^{-5}$ & 3.20 \\
8 & No & HH-ADS & 389,800 & 57,100 & $<1.0 \times 10^{-5}$ & 3.01 \\
8 & No & History Heuristic & 567,900 & 97,000 & $<1.0 \times 10^{-5}$ & 1.99 \\
\hline \hline 4 & Yes & History-ADS & 3681 & 1138 & $<1.0 \times 10^{-5}$ & 0.94 \\
4 & Yes & HH-ADS & 3974 & 1274 & $1.9 \times 10^{-4}$ & 0.79 \\
4 & Yes & History Heuristic & 4010 & 1206 & $<1.0 \times 10^{-5}$ & 0.77 \\
\hline 6 & Yes & History-ADS & 34,400 & 12,400 & $<1.0 \times 10^{-5}$ & 1.15 \\
6 & Yes & HH-ADS & 35,700 & 13,700 & $<1.0 \times 10^{-5}$ & 1.10 \\
6 & Yes & History Heuristic & 42,800 & 14,500 & $<1.0 \times 10^{-5}$ & 0.83 \\
\hline 8 & Yes & History-ADS & 293,900 & 100,000 & $<1.0 \times 10^{-5}$ & 1.39 \\
8 & Yes & HH-ADS & 334,900 & 153,300 & $<1.0 \times 10^{-5}$ & 1.28 \\
8 & Yes & History Heuristic & 533,600 & 265,200 & $<1.0 \times 10^{-5}$ & 0.80 \\
\hline
\end{tabular}

Our results for the Virus Game are presented in Table 7.4. Yet again, we observed the same pattern as in Othello, Relaxed Checkers, and Focus, with no deviation from the established pattern. The History-ADS heuristic performed best in all cases, up to $29 \%$ better in the 6-ply midgame case, with a $\mathrm{NC}$ of $5,872,700$ compared to the History Heuristic's 8,193,900. Again, each technique's benefit is to a very strong level of statistical certainty, with very large Effect Sizes over 2.0 in all cases, and a lowered Effect Size in the case of the History Heuristic. 
Table 7.3: Results comparing the History-ADS, the HH-ADS, and the History Heuristic in Focus.

\begin{tabular}{|c||c||c||c||c||c||c|}
\hline \hline Ply & Mid & Method & Avg. NC & Std. Dev. & P-Value & Effect \\
\hline 4 & No & History-ADS & $1,264,100$ & 90,900 & $<1.0 \times 10^{-5}$ & 10.46 \\
4 & No & HH-ADS & $1,346,300$ & 92,100 & $<1.0 \times 10^{-5}$ & 10.24 \\
4 & No & History Heuristic & $1,978,600$ & 221,500 & $<1.0 \times 10^{-5}$ & 8.59 \\
\hline 4 (Multi) & No & History-ADS & $2,150,300$ & 164,900 & $<1.0 \times 10^{-5}$ & 4.92 \\
4 (Multi) & No & HH-ADS & $2,239,800$ & 205,000 & $<1.0 \times 10^{-5}$ & 4.82 \\
4 (Multi) & No & History Heuristic & $3,358,000$ & 350,800 & $<1.0 \times 10^{-5}$ & 3.69 \\
\hline \hline 4 & Yes & History-ADS & $2,388,700$ & 631,400 & $<1.0 \times 10^{-5}$ & 2.37 \\
4 & Yes & HH-ADS & $2,595,700$ & 817,800 & $<1.0 \times 10^{-5}$ & 2.31 \\
4 & Yes & History Heuristic & $3,495,200$ & $1,035,000$ & $<1.0 \times 10^{-5}$ & 2.05 \\
\hline 4 (Multi) & Yes & History-ADS & $3,156,400$ & $1,695,300$ & $<1.0 \times 10^{-5}$ & 1.31 \\
4 (Multi) & Yes & HH-ADS & $3,448,600$ & $1,607,000$ & $<1.0 \times 10^{-5}$ & 1.28 \\
4 (Multi) & Yes & History Heuristic & $5,054,900$ & $3,008,900$ & $<1.0 \times 10^{-5}$ & 1.09 \\
\hline
\end{tabular}

Finally, Table 7.5 contains our results for Chinese Checkers. In this game, while the History Heuristic was always outperformed, the HH-ADS approach achieved better results than the original History-ADS heuristic in half the cases; the four-player midgame case, and six-player initial board position case. However, as with the other game models, in all cases the History-ADS heuristic and HH-ADS performed almost identically, with the largest difference being $17 \%$ in the six-player, midgame state. Yet again, all techniques produced an improvement in terms of $\mathrm{NC}$ with a very strong statistical certainty.

Our detailed discussion of these results is provided later in this chapter, in Section 7.9 . 
Table 7.4: Results comparing the History-ADS, the HH-ADS, and the History Heuristic in the Virus Game.

\begin{tabular}{|c||c||c||c||c||c||c|}
\hline \hline Ply & Mid & Method & Avg. NC & Std. Dev. & P-Value & Effect \\
\hline 4 & No & History-ADS & 154,400 & 20,800 & $<1.0 \times 10^{-5}$ & 3.47 \\
4 & No & HH-ADS & 161,500 & 17,200 & $<1.0 \times 10^{-5}$ & 3.21 \\
4 & No & History Heuristic & 194,900 & 25,100 & $<1.0 \times 10^{-5}$ & 2.05 \\
\hline 6 & No & History-ADS & $4,649,000$ & 767,100 & $<1.0 \times 10^{-5}$ & 4.60 \\
6 & No & HH-ADS & $4,687,500$ & 740,400 & $<1.0 \times 10^{-5}$ & 4.58 \\
6 & No & History Heuristic & $6,857,600$ & $1,075,300$ & $<1.0 \times 10^{-5}$ & 2.86 \\
\hline \hline 4 & Yes & History-ADS & 186,800 & 23,100 & $<1.0 \times 10^{-5}$ & 3.00 \\
4 & Yes & HH-ADS & 193,900 & 28,200 & $<1.0 \times 10^{-5}$ & 2.82 \\
4 & Yes & History Heuristic & 210,900 & 25,300 & $<1.0 \times 10^{-5}$ & 2.40 \\
\hline 6 & Yes & History-ADS & $5,872,700$ & 862,900 & $<1.0 \times 10^{-5}$ & 3.55 \\
6 & Yes & HH-ADS & $5,988,500$ & 772,700 & $<1.0 \times 10^{-5}$ & 3.49 \\
6 & Yes & History Heuristic & $8,193,900$ & $1,075,300$ & $<1.0 \times 10^{-5}$ & 2.36 \\
\hline
\end{tabular}

\subsection{Implementing the Killer Moves heuristic in the context of the History-ADS Heuristic}

The concept of multi-level ADSs, explored in the previous chapter, was based directly on the established Killer Moves heuristic, first discussed in Chapter 2. The Killer Moves heuristic seeks to limit the impact of cuts made on different levels of the tree to the ordering, by completely restricting information to a single level of the tree [54]. This heuristic retains a small number of moves at each level of the tree, which have been found to be good (i.e., produced a cut on that ply level), and which are explored first, if applicable, when moves are generated at each level of the tree.

The technique maintains a small number of moves, which produced cuts at each level of the tree. Generally, only one or two are recalled, and it simultaneously retains only the latest moves which have produced a cut, without employing counters. The 
Table 7.5: Results comparing the History-ADS, the HH-ADS, and the History Heuristic in Chinese Checkers.

\begin{tabular}{|c||c||c||c||c||c||c|}
\hline \hline Ply & Mid & Method & Avg. NC & Std. Dev. & P-Value & Effect \\
\hline 4 (4-play) & No & History-ADS & 475,600 & 124,700 & $<1.0 \times 10^{-5}$ & 2.16 \\
4 (4-play) & No & HH-ADS & 488,700 & 136,500 & $<1.0 \times 10^{-5}$ & 2.13 \\
4 (4-play) & No & History Heuristic & 550,100 & 147,400 & $<1.0 \times 10^{-5}$ & 1.98 \\
\hline 4 (6-play) & No & History-ADS & $1,282,500$ & 368,000 & $<1.0 \times 10^{-5}$ & 1.90 \\
4 (6-play) & No & HH-ADS & $1,219,800$ & 310,700 & $<1.0 \times 10^{-5}$ & 1.96 \\
4 (6-play) & No & History Heuristic & $1,546,700$ & 445,100 & $<1.0 \times 10^{-5}$ & 1.66 \\
\hline \hline 4 (4-play) & Yes & History-ADS & $1,236,600$ & 335,000 & $<1.0 \times 10^{-5}$ & 2.25 \\
4 (4-play) & Yes & HH-ADS & $1,176,900$ & 311,400 & $<1.0 \times 10^{-5}$ & 2.32 \\
4 (4-play) & Yes & History Heuristic & $1,589,000$ & 413,500 & $<1.0 \times 10^{-5}$ & 1.88 \\
\hline 4 (6-play) & Yes & History-ADS & $3,200,100$ & 817,900 & $<1.0 \times 10^{-5}$ & 1.92 \\
4 (6-play) & Yes & HH-ADS & $3,328,500$ & 918,000 & $<1.0 \times 10^{-5}$ & 1.87 \\
4 (6-play) & Yes & History Heuristic & $3,938,600$ & $1,089,900$ & $<1.0 \times 10^{-5}$ & 1.64 \\
\hline
\end{tabular}

similarities between the History-ADS heuristic, employing a multi-level ADS, and the Killer Moves heuristic are thus intuitively obvious. In fact, Killer Moves is equivalent to a specific configuration of the History-ADS. Specifically, this is the History-ADS heuristic, employing a multi-level ADS, with a limit on the maximum length of each ADS being either one or two, if the Move-to-Front rule is employed. An example of how this is accomplished in a specific execution is shown in Figure 7.2.

Given that employing a multi-level ADS did not perform as well as a single ADS, and a strict limit on the length produced even worse performance, we have a strong reason to suspect that the History-ADS heuristic can, in fact, outperform the established Killer Moves heuristic. If it were the case that it could outperform both the History Heuristic and Killer Moves, this would, indeed, be a very strong demonstration of its strengths. This motivation renders such a comparison worthwhile. 


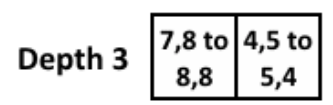

\begin{tabular}{c|c|c|}
\hline Depth 2 & \begin{tabular}{c|c}
3,2 to & 6,7 to \\
5,1 & 6,6 \\
\hline
\end{tabular} \\
\hline
\end{tabular}

Depth $1 \quad \begin{gathered}1,3 \text { to } \\ 1,6\end{gathered}$

Depth 3 \begin{tabular}{c|c|}
\hline 7,8 to & 4,5 to \\
8,8 & 5,4 \\
\hline
\end{tabular}

Depth $\mathbf{2}$\begin{tabular}{|c|c|c|}
\hline 1,1 to & $\mathbf{3 , 2}$ to & 6,7 to \\
4,1 & $\mathbf{5 , 1}$ & 6,6 \\
\hline
\end{tabular}

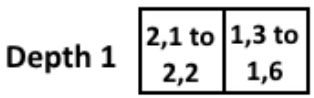

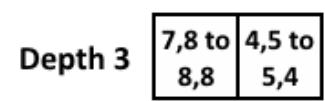

\begin{tabular}{c|c|c|}
\hline Depth 2 & \begin{tabular}{c|c|}
3,2 to & 6,7 to \\
5,1 & 6,6 \\
\hline
\end{tabular} \\
\hline
\end{tabular}

\begin{tabular}{c|c|c|}
\hline Depth 1 & 2,1 to & $\mathbf{1 , 3}$ to \\
2,2 & $\mathbf{1 , 6}$ \\
\hline
\end{tabular}

\begin{tabular}{c|c|c|}
\hline Depth 2 & 1,1 to & 3,2 to \\
4,1 & 5,1 \\
\hline
\end{tabular}

Depth 1 \begin{tabular}{|c|c|}
\hline 2,1 to & 1,3 to \\
2,2 & 1,6 \\
\hline
\end{tabular}

Figure 7.2: A demonstration of how the Killer Moves technique can be implemented using an ADS. Two, or fewer, moves are maintained initially at the three provided depths. The move $(2,1)$ to $(2,2)$ produces a cut at depth 1 , and so it is moved to the head of that list. Next, the move $(1,1)$ to $(4,1)$ produces a cut at depth 2 , so it is added to the list and moved to the front, and the move $(6,7)$ to $(6,6)$ is forgotten. Lastly, the move $(7,8)$ to $(8,6)$ produces a cut at depth 3 , and so it is placed at the head of the list, and $(4,5)$ to $(5,4)$ is forgotten. This process is identical to what the Killer Moves technique achieves.

\subsection{Experimental Model}

Our experiments involving the Killer Moves heuristic are identical to those already presented in this chapter for the History Heuristic, as in both cases, we are comparing the performance of the History-ADS heuristic to an established, previously-specified method. In each of our game models and configurations we will compare the original, single ADS version of the History-ADS heuristic and the Killer Moves heuristic, recording the average $\mathrm{NC}$ in each case. 
When we originally presented results for multi-level ADSs in the previous chapter, we limited the cases examined to a subset, specifically those with a ply depth greater than four. This was done because cuts cannot take place at the leaf or root nodes. Thus, in the four ply case, there exists only a single MIN ply and a single MAX ply where cuts can possibly take place. As the History-ADS, following the model of the History Heuristic, maintains separate data structures for MIN and MAX nodes, there would be no functional difference between a multi-level ADS and a single ADS. As opposed to this, in the case of Killer Moves, we will present results for all possible cases, as it was originally specified for the general case.

In this context, we allow the Killer Moves heuristic to maintain two moves per level, rather than only one. Additionally, we record the average NC, and employ the Mann-Whitney test for statistical significance, and also provide the corresponding Effect Sizes.

\subsection{Results for Killer Moves}

Table 7.6 presents our results comparing the Killer Moves and the History-ADS heuristic in the case of Othello. We observed that, at each ply depth from four to eight, and at both the initial and midgame states, the History-ADS heuristic outperformed Killer Moves, although both techniques generated similar performance, when compared to values when no ADS was involved, as reported in the previous chapter. For example, in the 6-ply initial board position case, the History-ADS achieved an NC of 3, 727, Killer Moves yielded a value of 4,013, and no ADS produced an NC of 5,061. We observed that in all cases, similar to the previous experiments, both the HistoryADS and the Killer Moves produced a statistically significant gain in tree pruning,

with an Effect Size ranging between 0.52 and 0.75, indicating a moderate to strong effect. The Effect Size for Killer Moves was slightly smaller, representing its slightly worse performance. 
Table 7.6: Results comparing the History-ADS and Killer Moves in Othello.

\begin{tabular}{|c||c||c||c||c||c||c|}
\hline \hline Ply & Mid & Method & Avg. NC & Std. Dev. & P-Value & Effect \\
\hline 4 & No & History-ADS & 525 & 175 & $3.2 \times 10^{-4}$ & 0.70 \\
4 & No & Killer Moves & 560 & 180 & $5.7 \times 10^{-3}$ & 0.53 \\
\hline 6 & No & History-ADS & 3727 & 1552 & $1.7 \times 10^{-3}$ & 0.56 \\
6 & No & Killer Moves & 4013 & 1720 & 0.015 & 0.44 \\
\hline 8 & No & History-ADS & 23,600 & 10,800 & $<1.0 \times 10^{-5}$ & 0.75 \\
8 & No & Killer Moves & 28,400 & 15,000 & $3.3 \times 10^{-3}$ & 0.68 \\
\hline \hline 4 & Yes & History-ADS & 1701 & 570 & $2.7 \times 10^{-4}$ & 0.67 \\
4 & Yes & Killer Moves & 1788 & 631 & $2.9 \times 10^{-3}$ & 0.55 \\
\hline 6 & Yes & History-ADS & 13,300 & 6916 & $7.0 \times 10^{-5}$ & 0.69 \\
6 & Yes & Killer Moves & 15,500 & 6696 & 0.015 & 0.47 \\
\hline 8 & Yes & History-ADS & 92,900 & 50,000 & $<1.0 \times 10^{-5}$ & 0.79 \\
8 & Yes & Killer Moves & 113,700 & 66,500 & $1.4 \times 10^{-4}$ & 0.64 \\
\hline
\end{tabular}

Table 7.7 holds our results comparing the History-ADS and Killer Moves in the context of Relaxed Checkers. We again see a consistent pattern emerging in the data. The History-ADS heuristic outperformed the Killer Moves technique in all situations, and in some cases, particularly in the 8-ply case, by a noticeable margin. For example, the History-ADS performed 33\% better than Killer Moves in the 8-ply midgame case, comparing a $\mathrm{NC}$ of 293, 000 to 440,000 . We observed a very statistically strong impact in all cases, with a range of effect sizes between 0.66 and the very high value of 3.55.

Considering Table 7.8, we see the trend continue, with the History-ADS heuristic outperforming the Killer Moves technique in all cases. Although Focus has a much larger branching factor than Relaxed Checkers, with a much higher NC, the results were quite a bit closer in this case, with the History-ADS performing $11 \%$ better than Killer Moves in the two-player, initial case (NCs of 1,421,300 and 1,264,300, respectively), and all others being closer. The possible reasons for this will be explored later in this chapter. The same trend was observed as with the previous games, 
Table 7.7: Results comparing the History-ADS and Killer Moves in Relaxed Checkers.

\begin{tabular}{|c||c||c||c||c||c||c|}
\hline \hline Ply & Mid & Method & Avg. NC & Std. Dev. & P-Value & Effect \\
\hline 4 & No & History-ADS & 4649 & 493 & $<1.0 \times 10^{-5}$ & 1.49 \\
4 & No & Killer Moves & 4927 & 529 & $<1.0 \times 10^{-5}$ & 1.16 \\
\hline 6 & No & History-ADS & 41,000 & 5588 & $<1.0 \times 10^{-5}$ & 3.55 \\
6 & No & Killer Moves & 52,200 & 6723 & $<1.0 \times 10^{-5}$ & 2.50 \\
\hline 8 & No & History-ADS & 358,400 & 53,200 & $<1.0 \times 10^{-5}$ & 3.20 \\
8 & No & Killer Moves & 470,300 & 87,800 & $<1.0 \times 10^{-5}$ & 2.55 \\
\hline \hline 4 & Yes & History-ADS & 3681 & 1138 & $<1.0 \times 10^{-5}$ & 0.94 \\
4 & Yes & Killer Moves & 4213 & 1126 & $6.0 \times 10^{-4}$ & 0.66 \\
\hline 6 & Yes & History-ADS & 34,400 & 12,400 & $<1.0 \times 10^{-5}$ & 1.15 \\
6 & Yes & Killer Moves & 39,100 & 14,700 & $<1.0 \times 10^{-5}$ & 0.97 \\
\hline 8 & Yes & History-ADS & 293,100 & 100,000 & $<1.0 \times 10^{-5}$ & 1.39 \\
8 & Yes & Killer Moves & 440,000 & 231,400 & $<1.0 \times 10^{-5}$ & 1.02 \\
\hline
\end{tabular}

with both techniques generating an improvement to $\mathrm{NC}$ with a very high margin of statistical certainty, and a large Effect Size.

Our results for the Virus Game are presented in Table 7.9. As before, while the performance was similar, as in the case with Focus, the History-ADS heuristic outperformed the Killer Moves technique in all cases, at both the midgame and initial board positions. A typical example is seen in the 6-ply initial board position, where the History-ADS performed $10 \%$ better than Killer Moves, given the figures.

Lastly, Table 7.10, containing our results for Chinese Checkers, continues the trend. In this case, the results for the History-ADS heuristic and Killer Moves were very close to each other, especially in the midgame case, with six players, where the performances were almost identical. Otherwise, the History-ADS performed better in every case. 
Table 7.8: Results comparing the History-ADS and Killer Moves in Focus.

\begin{tabular}{|c||c||c||c||c||c||c|}
\hline \hline Ply & Mid & Method & Avg. NC & Std. Dev. & P-Value & Effect \\
\hline 4 & No & History-ADS & $1,264,300$ & 90,900 & $<1.0 \times 10^{-5}$ & 10.46 \\
4 & No & Killer Moves & $1,421,300$ & 105,100 & $<1.0 \times 10^{-5}$ & 10.04 \\
\hline 4 (Multi) & No & History-ADS & $2,150,300$ & 164,900 & $<1.0 \times 10^{-5}$ & 4.92 \\
4 (Multi) & No & Killer Moves & $2,224,500$ & 175,000 & $<1.0 \times 10^{-5}$ & 4.84 \\
\hline \hline 4 & Yes & History-ADS & $2,388,700$ & 631,400 & $<1.0 \times 10^{-5}$ & 2.37 \\
4 & Yes & Killer Moves & $2,678,400$ & 648,000 & $<1.0 \times 10^{-5}$ & 2.29 \\
\hline 4 (Multi) & Yes & History-ADS & $3,156,400$ & $1,695,300$ & $<1.0 \times 10^{-5}$ & 1.31 \\
4 (Multi) & Yes & Killer Moves & $3,258,700$ & $1,525,400$ & $<1.0 \times 10^{-5}$ & 1.30 \\
\hline
\end{tabular}

\subsection{Discussion}

The results we have presented in this chapter demonstrate the power of the HistoryADS heuristic, even when compared to the established, highly-regarded techniques, specifically, the Killer Moves strategy, and the History Heuristic. We found that in nearly every case examined, the History-ADS heuristic outperformed both of these established techniques, or performed on a level comparable to them.

The discussion is more meaningful when we observe that the HH-ADS, a variation of the History-ADS heuristic, which employed a specially-configured ADS, was designed to emulate the History Heuristic's update mechanism. Indeed, this was achieved through modifying the Move-Ahead- $k$ rule so that moves are transposed a number of positions towards the front in relation to the level of the tree in which they produced a cut, to limit the impact of cuts near the bottom of the tree. However, in nearly every case examined, the simple Move-to-Front rule outperformed the HHADS. The HH-ADS did outperform the Move-to-Front rule in the context of Chinese Checkers, in some cases, and was generally very close, but it was reliably outmatched overall. Furthermore, to our surprise, the History Heuristic, which is highly acclaimed itself, performed even worse than HH-ADS, in some cases by a very large amount, 
Table 7.9: Results comparing the History-ADS and Killer Moves in the Virus Game.

\begin{tabular}{|c||c||c||c||c||c||c|}
\hline \hline Ply & Mid & Method & Avg. NC & Std. Dev. & P-Value & Effect \\
\hline 4 & No & History-ADS & 154,400 & 20,800 & $<1.0 \times 10^{-5}$ & 3.47 \\
4 & No & Killer Moves & 173,400 & 21,600 & $<1.0 \times 10^{-5}$ & 2.80 \\
\hline 6 & No & History-ADS & $4,649,000$ & 767,100 & $<1.0 \times 10^{-5}$ & 4.60 \\
6 & No & Killer Moves & $5,211,200$ & 858,600 & $<1.0 \times 10^{-5}$ & 4.16 \\
\hline \hline 4 & Yes & History-ADS & 186,800 & 23,100 & $<1.0 \times 10^{-5}$ & 3.00 \\
4 & Yes & Killer Moves & 210,100 & 17,000 & $<1.0 \times 10^{-5}$ & 2.42 \\
\hline 6 & Yes & History-ADS & $5,872,700$ & 862,900 & $<1.0 \times 10^{-5}$ & 3.55 \\
6 & Yes & Killer Moves & $6,380,100$ & 990,900 & $<1.0 \times 10^{-5}$ & 3.29 \\
\hline
\end{tabular}

failing even to gain a statistically significant reduction in $\mathrm{NC}$ in the case of six-player Othello, when the measurements were taken from the initial board position.

Although we were somewhat surprised that the History Heuristic was outperformed by the History-ADS heuristic in every case examined, reviewing our results from the previous chapters and its relative performance to the HH-ADS, such an outcome is rather predictable. We had earlier observed that, in the context of the History-ADS heuristic, the Move-to-Front rule consistently outperformed the less sensitive Transposition rule. In this context, since the HH-ADS transposes elements towards the head of the list a variable number of spaces, it occupies a middle ground between the Move-to-Front rule and the Transposition rule, in terms of sensitivity. Thus, although it attempts to more intelligently manage the qualities of the game tree, our results support the conclusion that the History-ADS heuristic benefits most from adapting as quickly as possible.

The strategy of the History Heuristic, however, is even less sensitive to change. This is because the ranking of the moves will only change when one move's counter exceeds another. As opposed to this, the HH-ADS changes its structure by some amount every time it is queried. Furthermore, our results show that the History Heuristic allows very strong moves to gain a substantial lead over all others. This is 
Table 7.10: Results comparing the History-ADS and Killer Moves in Chinese Checkers.

\begin{tabular}{|c||c||c||c||c||c||c|}
\hline \hline Ply & Mid & Method & Avg. NC & Std. Dev. & P-Value & Effect \\
\hline 4 (4-play) & No & History-ADS & 475,600 & 124,700 & $<1.0 \times 10^{-5}$ & 2.16 \\
4 (4-play) & No & Killer Moves & 490,000 & 126,500 & $<1.0 \times 10^{-5}$ & 2.13 \\
\hline 4 (6-play) & No & History-ADS & $1,282,400$ & 368,000 & $<1.0 \times 10^{-5}$ & 1.90 \\
4 (6-play) & No & Killer Moves & $1,305,100$ & 341,500 & $<1.0 \times 10^{-5}$ & 1.88 \\
\hline \hline 4 (4-play) & Yes & History-ADS & $1,236,600$ & 335,000 & $<1.0 \times 10^{-5}$ & 2.25 \\
4 (4-play) & Yes & Killer Moves & $1,268,400$ & 380,900 & $<1.0 \times 10^{-5}$ & 2.22 \\
\hline 4 (6-play) & Yes & History-ADS & $3,200,100$ & 817,900 & $<1.0 \times 10^{-5}$ & 1.92 \\
4 (6-play) & Yes & Killer Moves & $3,195,000$ & 799,100 & $<1.0 \times 10^{-5}$ & 1.92 \\
\hline
\end{tabular}

in contrast to the case of the HH-ADS, where the move cannot go beyond the head of the list. Indeed, when viewing the History Heuristic's internal updates as the search proceeded, in both the Virus Game and Othello, a single move would quickly gain a nearly insurmountable lead. This is the likely reason for the History Heuristic's poor performance, compared to the History-ADS heuristic, and the HH-ADS. This is also consistent with our previous observations. Our results strongly suggest that the History-ADS heuristic outperforms the History Heuristic under a broad set of board games, and we hypothesize, based on these results, that it is likely to do so in others as well.

The fact that the single ADS, Move-to-Front History-ADS heuristic outperforms the Killer Moves strategy is not surprising, and is exactly in line with our hypothesis. Indeed, we had earlier determined that a single ADS would generally outperform a multi-level ADS, and that a multi-level ADS with a restriction on its length would have its performance hampered even further. As the Killer Moves technique is identical, in spirit, to the History-ADS with a multi-level ADS, and with a limit of two on the length, we would expect it to be outmatched by the single, unbounded ADS. Our results, clearly, support that. 
The degree by which the Killer Moves technique was outperformed varied between the various game models, with it doing best in Chinese Checkers, and worst in Relaxed Checkers. Given that it can only maintain a very small number of moves, this suggests that storing more information achieves a superior move ordering in the case of Relaxed Checkers, but it is not so critical in the case of Chinese Checkers, with the other games falling between these extreme cases. This is consistent with our findings for bounding the length of the ADS in the previous chapter, and demonstrates the benefits of testing techniques on a range of game models.

\subsection{Chapter Conclusions}

Our work in this chapter demonstrates that the History-ADS heuristic is able to produce strong gains in terms of tree pruning. Additionally, it is also capable of outperforming the established Killer Moves technique and History Heuristic in a wide range of game models and configurations. This is a particularly strong result, which serves to justify its usage in game playing engines, particularly given its lightweight qualities, and the fact that it does not need any additional sorting.

Our results further reinforce the idea that, in the context of the History-ADS heuristic, the most basic configuration tends to perform best. We confirm this because the single, unbound, Move-to-Front implementation of the History-ADS outperformed both established heuristics, as well as one that attempted to model the History Heuristic in the context of ADSs. This suggests that within the perspective of move ordering, that is based on a move history criterion, a simpler mechanism is, in fact, superior!

The next chapter concludes the thesis, summarizing our contributions in achieving move ordering through opponent threat and move history. The chapter also suggests avenues for future research. 


\section{Chapter 8}

\section{Summary, Contributions, and Future Work}

In this work, we have extensively explored the application of ADSs to the field of game playing, particularly in the context of move ordering, in deterministic approaches based on the alpha-beta search. We have verified, in a wide range of situations and game models, in both two-player and multi-player games, that the use of ADSs can achieve significant benefits in terms of tree pruning, translating to a smaller search space. In this final chapter, we summarize the conclusions of the thesis as a whole, the contributions of our work to the field, and discuss potential limitations of our investigation as well as avenues for future work.

\subsection{Summary by Chapters}

Our investigation was inspired, at its outset, by the observation that multi-player games remain a relatively unexplored topic within game playing literature, despite the prevalence of games involving more than two players in the real world. Acknowledging that strategies for multi-player games generally have difficulty performing on a level 
comparable with their two-player counterparts, we turned to the field of ADSs, with the objective of inspiring novel approaches to this problem. The work presented in this thesis represents the first application of ADS-based techniques to the field of intelligent game play.

Chapter 2: This chapter presented our survey of the field of two-player and multiplayer game playing. We discussed state-of-the-art and traditional approaches, with a particular emphasis on the alpha-beta technique, and the move ordering strategies to enhance the latter. We also presented a survey of the field of ADSs, and the techniques from that domain that were to be employed in the subsequent chapters as an "improving agent".

Chapter 3: Initially, we focused our attention on the Best-Reply Search (BRS), as it is a relatively new, state-of-the-art multi-player game playing technique, that was found to outperform previously-known methods in a variety of game models [52]. We first presented the idea of using an ADS as a ranking mechanism within the context of game playing, and specifically, incorporating it into the concept of ranking opponents based on their relative threats. This knowledge was then applied to the BRS to achieve improved move ordering, leading to the design and implementation of the Threat-ADS heuristic. Our results clearly determine that, despite employing only a very small ADS, the Threat-ADS is able to achieve statistically significant improvements, in terms of the Node Count (NC), in each game model. Furthermore, it was able to maintain these results, in general, with variations on both the number of players, and at midgame board positions.

Chapter 4: Based on our success with the Threat-ADS heuristic, we next strove to address a number of unanswered questions. Specifically, we extended our experiments to deal with variations on the update mechanism employed by the ADS, given the wide range of varied update mechanisms reported in ADS literature, and their differing qualities. We also examined the performance of the Threat-ADS heuristic in deeper game trees, and compared absorbing update mechanisms with the more common ergodic variety. We found that the Threat-ADS' performance did not deteriorate 
in deeper trees, and that all of the ergodic update mechanisms examined performed very similarly, although, as was originally hypothesized, the absorbing strategies did not produce noticeable improvements in tree pruning.

Chapter 5: Given that the Threat-ADS heuristic was able to achieve a consistent, statistically significant reduction in tree size, in a wide range of cases, only employing an extremely small adaptive list (often no more than three elements), we determined that it was worthwhile to investigate the applicability of ADSs in the context of two-player game playing, as well. We initiated this investigation by presenting a method by which an ADS can be used to achieve move ordering via the ranking of the moves themselves, similar in concept to the Threat-ADS' ranking by opponent. We specified the History-ADS heuristic, which makes use of this strategy, to examine this concept. Our experiments confirmed that, in all two-player and multi-player games examined, in a variety of ply depths and at both initial and midgame positions, the History-ADS was able to achieve substantial improvements in terms of the NC. Generally, we determined that the more sensitive Move-to-Front update mechanism outperformed the Transposition rule, indicating that a larger change to the list's structure is generally beneficial to the History-ADS heuristic.

Chapter 6: While the History-ADS heuristic was proven to be very powerful, it possessed some qualities that could be limiting its performance, or causing it to waste time or space. Particularly, the History-ADS heuristic, as originally specified, applied all its insight equally to all levels of the game tree, with a list of an unbounded length, which could grow quite large if many different moves in the search produced cuts. We thus examined placing a limit on the length of the History-ADS' list, and using a different ADS for each level of the tree, which we referred to as a multi-level ADS. Our results demonstrated that even with a very small ADS list where the length was bounded by five, the History-ADS maintained a majority of its performance, thus proving that the History-ADS heuristic does not require a large data structure, or a potentially expensive list searching mechanism. The multi-level ADS strategy did not produce an improvement in performance, suggesting that applying learning at multiple levels of the tree is more important than prioritizing based on the ply level. 
Chapter 7: Based on our promising results with the History-ADS heuristic, we deemed it worthwhile to compare the History-ADS heuristic to two established and well-known move ordering heuristics, namely, the Killer Moves strategy, and the History Heuristic. We presented this comparison in this chapter, along with methods by which these strategies could be implemented, or approximated, using ADSs. We found, to some surprise, that the History-ADS heuristic, in its original form, performed better than either the Killer Moves strategy or the History Heuristic. While the original History-ADS heuristic outperformed our ADS-based approximation of the History Heuristic, the ADS-based version did outperform the original. These results reinforce the idea that larger adaptations are more beneficial than smaller ones in the context of history-based move ordering, and emphasize that in this domain, simpler strategies, are, in fact, superior.

\subsection{Contributions of the Thesis}

Our work has provided significant, and varied contributions to the field of game playing, over the course of our investigations. These are listed below:

- Our work represents the first combination of the fields of game playing, and ADSs. Prior to our research, no other method of applying ADSs to game playing, or to potential enhancements of alpha-beta related techniques, existed in the literature.

- We have presented new strategies, by which an ADS can be employed to order moves, in an effective and dynamic manner, in a game tree. This has led to statistically-significant improvements in tree pruning, in both two-player and multi-player games. 
- We have introduced the concept of employing opponent threats, in the context of a multi-player game, to order moves. While opponent threats are a known concept in multi-player game play, the idea of using them to enhance move ordering represents a novel approach.

- Employing the previous two ideas, we have specified and implemented the Threat-ADS heuristic, a general, domain independent, move ordering heuristic, for the state-of-the-art BRS. We have demonstrated that this can achieve statistically significant savings in terms of tree pruning, in a wide range of cases.

- Inspired by existing strategies to order moves in the game tree, based on their historical performance, we have specified the similarly domain-independent History-ADS heuristic for both two-player and multi-player games. We have experimentally proven its substantial capacity to reduce tree sizes.

- We have offered possible extensions to the History-ADS heuristic. This has been done by examining the possibility of limiting its memory impact, as well as prioritizing its information based on ply levels.

- Our work has described how the established Killer Moves strategy, and the History heuristic, can be related to ADSs. In particular, we have specified how the Killer Moves strategy can be implemented using ADSs, and how the History Heuristic can be approximated. We have named our ADS-based approximation of the History Heuristic as the HH-ADS. It too serves as a general-use move ordering strategy.

- We have demonstrated, in our final round of experiments, that the History-ADS heuristic is, in fact, able to outperform both the Killer Moves strategy, and the History Heuristic. 
- Apart from the conceptual contributions, with regard to the depth of experimentation, we have examined a very wide range of possible configurations, game models, and domains. This was done in order for us to demonstrate the applicability of our results in the general sense, and the power of our novel heuristics. These are as follows:

- The multi-player games Focus and Chinese Checkers, as well as the Virus Game of our own design;

- The two-player games Relaxed Checkers and Othello, and the two-player version of Focus;

- A range of ergodic update mechanisms, specifically the Move-to-Front, Transposition, Move-Ahead- $k$, and $\operatorname{POS}(k)$ strategies;

- Absorbing versus Ergodic ADS strategies;

- Variation on the number of players in multi-player games;

- Initial and midgame starting positions for the games;

- Variation on the memory limits applied to the History-ADS heuristic.

\subsection{Limitations and Future Work}

Exploring the intersection between two, formerly unrelated fields of Computer Science is an ambitious goal, and thus, by necessity, our work had to be restricted in some ways, both for time, and for space within the thesis itself. Furthermore, technology and resource limitations prevented us from exploring some avenues. However, these limitations also provide possible avenues of future research. These, along with other unexplored avenues, are listed below. 
- While we examined a substantial range of different games, as well as number of well-known and studied update mechanisms, additional insight could be gained by a large scale study, examining the Threat-ADS, History-ADS, and perhaps other ADS-based techniques in a truly large set of games. Certainly, there are enough known board games to provide such a vast testing environment, in both the two-player, and multi-player case. This could be the subject of a larger study, involving a number of researchers working in tandem on these individual game engines, and experiments.

- Our experiments in this work were limited by the capacity of a standard personal computer, thus limiting the ply depth of our search, particularly in those games possessing a particularly large branching factor, specifically Focus and Chinese Checkers. While our results strongly support the hypothesis that the ThreatADS and History-ADS heuristics maintain their performances as the ply depth of the search increases, it would be fascinating if their performances are studied in very deep trees. This could be accomplished by the use of a large, multiprocessor machine, which, unfortunately, was outside of our availability for this work.

- The Threat-ADS and History-ADS heuristics provide powerful, domain independent strategies, which can be employed to play a wide range of games to which the BRS, and alpha-beta search in general, apply. However, it is certainly the case that, in the context of well-studied games, such as Chess, domain dependent techniques, or specific refinements to general use strategies, are important, as well. Given our success with ADS-based techniques in the domain independent situation, it is worthwhile to investigate if they can be applied in domain-specific strategies as well, perhaps in terms of opponent modeling in a card game, such as Poker. Additionally, specific refinements to the ThreatADS or History-ADS heuristics may allow them to perform even better in the context of specific games, and when they are augmented by domain dependent heuristics. 
- The ADS-based techniques introduced in this work all deal with the idea of move ordering. However, while this is a natural application for a ranking mechanism, insight gained from ADSs may be applicable in other subfields of game playing as well. For example, consider the Max-N algorithm, described in Chapter 2. The Max-N algorithm, unlike alpha-beta for two-player games, may encounter meaningful ties, which in general, are broken to the left. However, considering an ADS that can rank opponents based on their relative threat, it may be more reasonable to break ties assuming each player will attempt to minimize his most powerful opponent, if two moves provide him with an equal value. This represents one possible application of an ADS outside move ordering.

- Consider the Monte-Carlo UCT algorithm, briefly discussed in Chapter 2. This strategy chooses which move is to be expanded based on its UCTValue, which could be very close in some contexts. It may be useful to provide an ADS to rank opponents, or possible moves, or even board states, as a secondary mechanism whenever two UCTValues are very close, or identical. Determining when to use the ADS, and when to employ the UCTValue, is itself an avenue of investigation, and we suggest that thresholds could be learned via any number of machine learning strategies. Research in this direction could be extremely promising.

- In this work, due to our desire to formulate a linear ranking, we focused our attention on adaptive lists. However, as described in Chapter 2, a wide range of ADSs exist for tree-based structures as well. Besides considering situations outside the direct ranking of move ordering, there may be applicability of tree-based ADSs to game playing as well. Alternatively, the "looser" ranking provided by the levels of a tree may have its advantages. The applicability of tree-based ADSs to game playing, as well as its potential performance, is currently an open question. 


\section{Appendix A}

\section{Appendix: Game Models}

\section{A.1 Overview}

In the course of this work, we have implemented a range of games, in order to properly test the ADS-based heuristics we have developed. This appendix specifies in detail the rules of the games we have chosen to employ, as well as the respective implementation decisions (such as their board evaluation metrics), that had to be made in order to create the test bed for our work. Specifically, we will discuss the Virus Game, a highly configurable testing game of our own creation, the games Focus and Chinese Checkers, as well as the two player games Othello, and Checkers or Draughts, which we have modified to Relaxed Checkers, to render the tests to be more meaningful. 


\section{A.2 The Virus Game}

The Virus Game is a "test-bed" game that may be played on an arbitrarily sized board by any number of players (at least two). This game is of our own creation, designed to be fast-running, and highly configurable. It is, however, based on similar games that have been discussed in the past, particularly those that model artificial life systems [1].

Rules of the Virus Game: The Virus Game is played on a $j$-by- $k$ sized gridshaped board, which may be of any arbitrary size, although it must at least be large enough to accommodate all the players. In the context of our experiments, we employed a 5-by-5 board, in order to force players to interact with a greater frequency. The number of players is any number $n$ where $n \geq 2$, and thus the Virus Game may be played as a two-player or a multi-player game. Each player begins with $m$ pieces, where again $m$ is a configurable parameter with $m \geq 1$.

Upon initialization (or setup) of the game, each of the players' $m$ pieces are placed randomly somewhere on the board. This decision to randomly place the pieces, was made after some experimentation. It was done so as to insure that the game did not play out in the same manner every time, thus guaranteeing that our move ordering heuristics would be tested under a varied range of possible game trees. In the context of other games studied in the game playing literature, this is usually compensated for in the game-playing algorithm with a small random factor [52]. However as we were the ones who designed the Virus Game, we took advantage of the opportunity to randomize starting positions directly. Figure A.1 shows a possible starting position for the Virus Game, on a 5-by-5 board with four players.

The play proceeds in a turn-based order around the "table", similar to most multiplayer combinatorial games, from the first player, who is usually determined randomly, again to avoid repetition. During a player's turn, he may "infect" any square that he controls, or is adjacent to a square that he controls. In this version of the game, we do not consider squares on the diagonal to be adjacent, however changing this should 


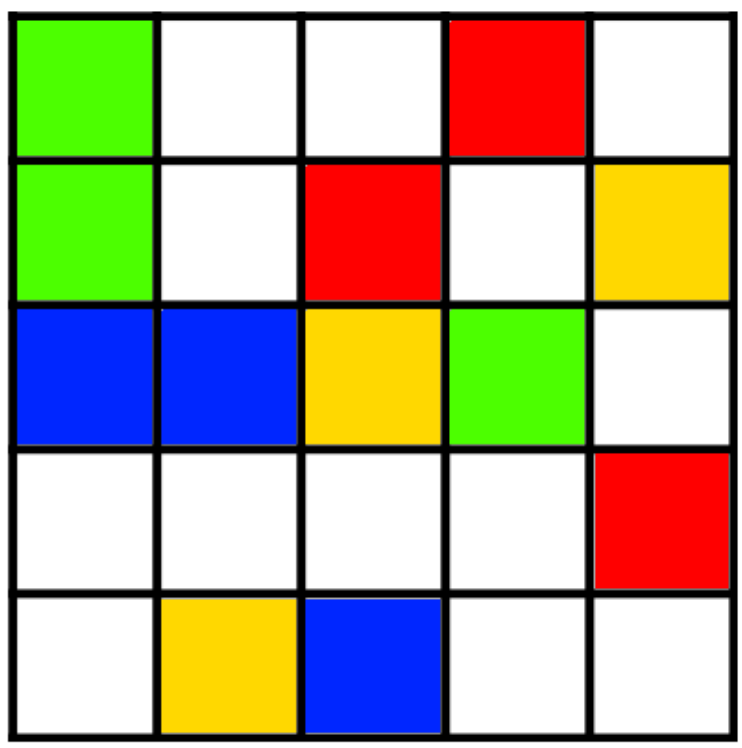

Figure A.1: The starting position for the Virus Game. Player 1 is red, and players 2, 3 , and 4 are blue, yellow, and green, respectively.

not change the properties of the game considerably. Only a single square may be infected like this, after which the play proceeds to the next player. The player claims the square that he has infected, if he did not already control it. He also can claim a number of adjacent squares, which are usually the ones immediately above, below, and to the right and left of the infected square. The squares claimed are configurable according to the player's "virulence", as discussed below. A sample move in the Virus Game, for the red player, is shown in Figure A.2.

When a player has no more pieces on the board, he is not able to make a move, and is thus considered to be eliminated, just as in the case of other territory control or piece capturing multi-player games. Play proceeds until only a single player remains, at which point that player is declared to be the Winner. 

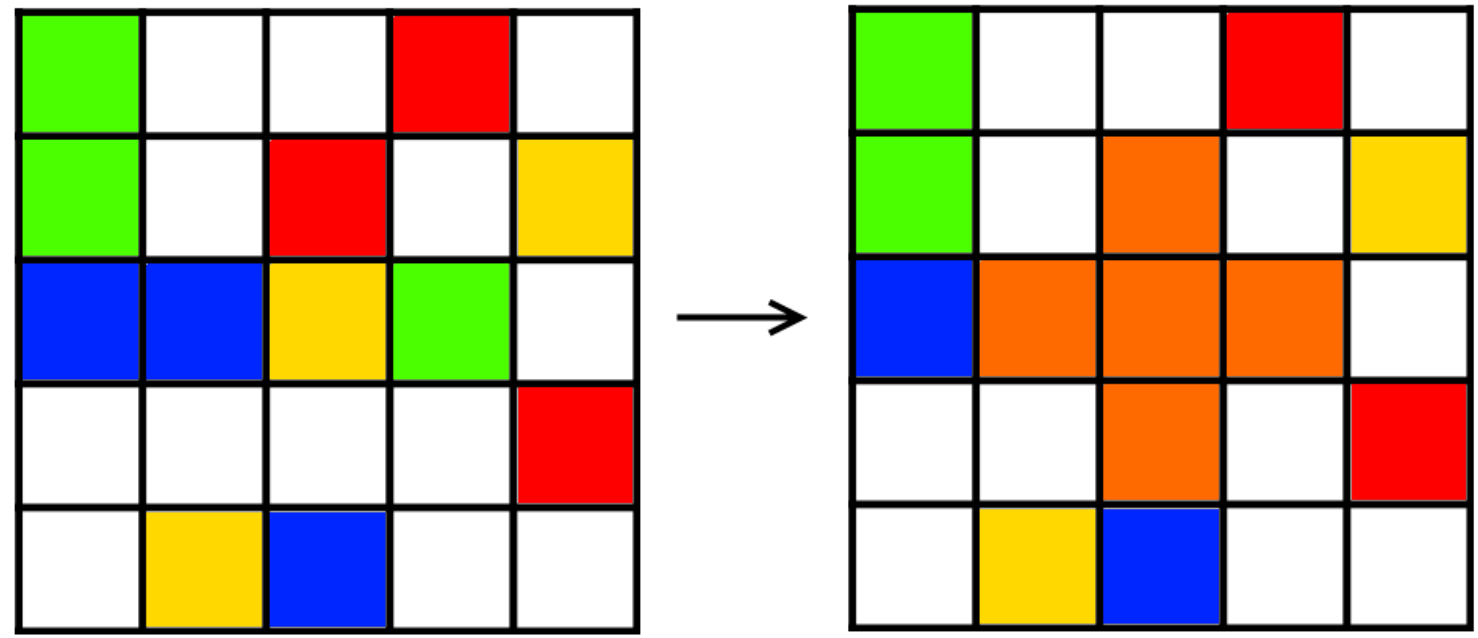

Figure A.2: A possible move for the red player in the Virus Game. The player has infected the central square and his claimed squares are coloured orange.

We observe that as a game, the Virus Game is not particularly exciting or challenging. Players can easily cancel each others' progress out by simply reclaiming the squares taken by their opponents, and nothing prevents them from doing this. In fact, should play come down to two players with equivalent virulences, optimal play will result in the two of them canceling each others moves perpetually, and the game will never end. However, the configurability of the model and its ease of implementation make it a useful game model when we are interested in measuring quantities other than which player actually wins the game.

Board Evaluation for the Virus Game: In order to allow for the game playing techniques described in this thesis to function within the context of our chosen game models, rather than simply developing software to allow play of the games in question, we must also decide upon and implement an evaluation heuristic function for each of them. 
Luckily, this task is not an onerous one in the context of the Virus Game, as it is observed to be a particularly simple game, by design, where the only move available to a player is to attempt to capture, or infect, more squares. As befits such a simple game, we employ the simple evaluation function of taking the value of the game state to be the number of squares that a player currently controls.

Virulence: As mentioned above, the squares a player claims when he infects a square, are configurable based on that player's virulence. A player's virulence determines how many squares, and which squares, he claims when he takes his turn. Therefore, the virulence provides a mechanism to configure the relative strength of the player, as a player with a "better" virulence will have more tactically rewarding options available to him than to others. Also, assuming virulence is the same between players, a different virulence specifies a different variant of the Virus Game. As the virulence is represented by a three-by-three grid of binary active or inactive squares, it is clear that a player's virulence may have between one and nine "active" squares. It is intuitive that a virulence with more active squares could be considered better than one that does not.

As well as the number of squares, the locations of those squares surrounding the central, infected square can contribute to the strength of a given virulence. For example, consider the two arrangements, each with four active squares, in Figure A.3. The virulence arrangement on the left has all of its active squares near the top of the grid, whereas the one on the right is more balanced. Therefore, a player using the left virulence would have issues attacking a player located below him on the board, while that same player would perform very well attacking a player above him. Thus, the player has a circumstantial advantage or disadvantage based on the current board position. The use of unbalanced virulences can necessitate different tactics within the context of the Virus Game. 

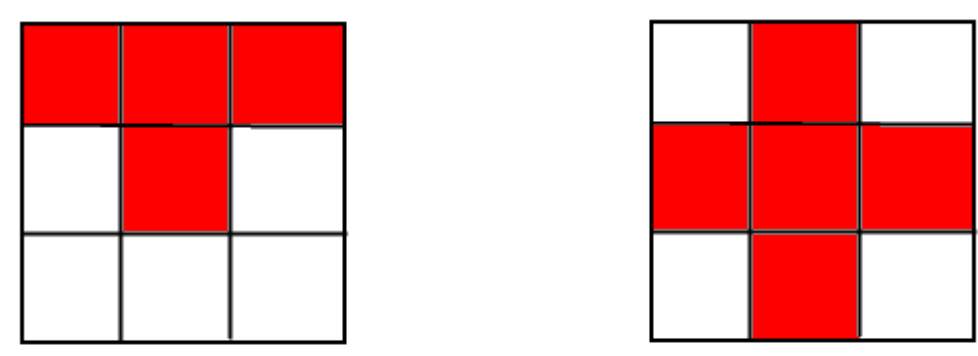

Figure A.3: A comparison between a top-heavy (unbalanced) virulence and a balanced virulence.

Given that all directions in the Virus Game are equivalent (as there are no fixed start positions, and all directions are functionally identical), while an unbalanced virulence can offer an advantage based on a board state, the direction being "unbalanced" should not matter. Thus, it is possible to rotate or flip a virulence without altering its functionality. We refer to two virulences that are equivalent through rotations and flips as being isomorphic. For example, consider the set of virulences in Figure A.4. All of these virulences are isomorphic to each other, and are thus considered functionally equivalent within the context of the Virus Game. This observation simplifies the need to explore a wide variety of virulence configurations, to achieve a given result. 

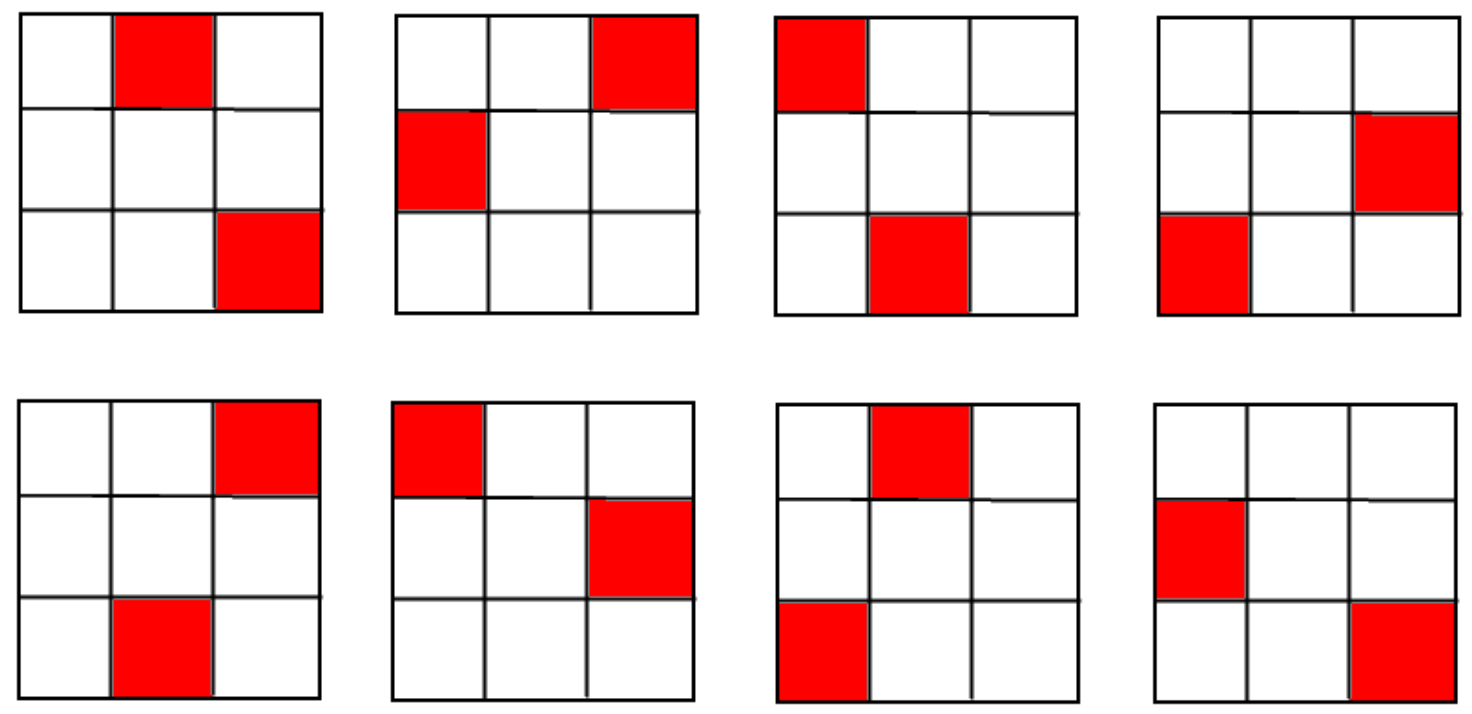

Figure A.4: A set of virulences that are considered to be "isomorphic" to each other.

\section{A.3 Focus}

Focus is a commercially-released board game for two to four players, first published in 1963 by Sackson, and subsequently re-released many times, including under the names Domination and Domino [50]. Focus is notable for its large branching factor, as well as for allowing some variety of moves that do not usually exist in grid-based board games, such as incorporating the third dimension into its possible moves and scoring system. As a result of these characteristics, Focus has seen application in the past as a testing environment for state-of-the-art game playing techniques [52].

Rules of Focus: Focus is played on an 8-by-8 board (in fact it was originally designed to be playable on a checkerboard, similar to Chess), by between two and four players. Unlike most checkerboard-based games, the corner three squares on each side are omitted, and players are not permitted to move pieces into or out of those squares. The setup for Focus depends on the number of players that are participating in the game, and the pieces are initially placed in one of three fixed positions, corresponding 
to that number. The positions for the two, three, and four player variants of the game are shown in Figure A.5. With some examination, it is clear that these positions are intended to be as fair as possible for each player.
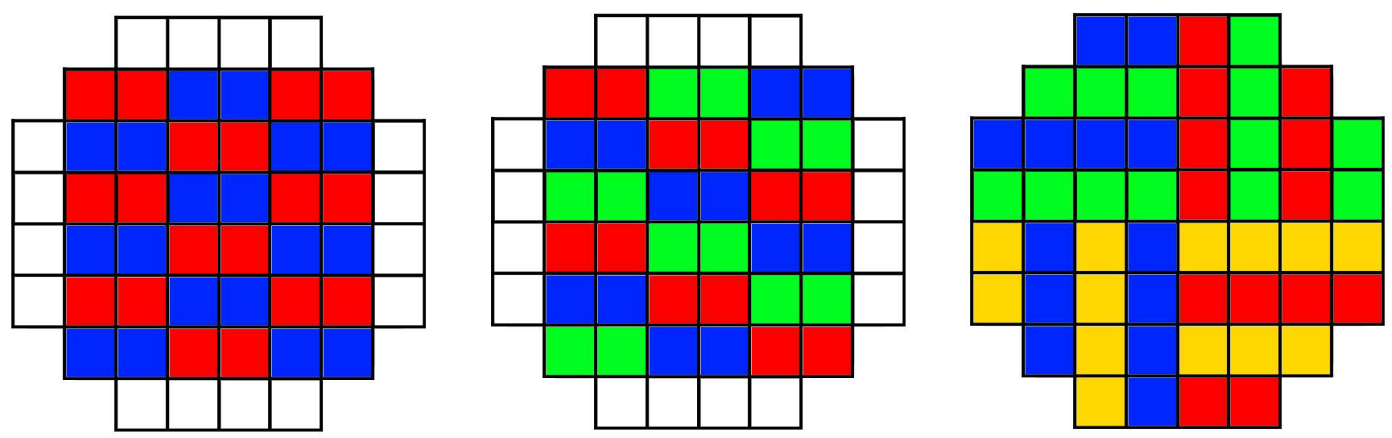

Figure A.5: The two, three, and four player starting positions for Focus.

Unlike most games of this type, Focus is complicated by the fact that pieces may be placed on top of each other, adding another dimension to the game's state space. The need for this three-dimensional game space must be accounted for in Focus' implementation, and thus, the implementation of Focus is substantially more involved than many other, simpler games. Furthermore, the Z-axis greatly increases the number of possible game positions and moves.

Play begins with a randomly chosen player, and then proceeds around the "table" in a clockwise manner. During his turn, a player may move any stack of pieces a number of squares orthogonally (vertically or horizontally), from its starting position, equal to the size of the stack, as long as he "controls" the stack. This "control" is determined, simply, by the player's piece occupying the topmost position on the stack. The player may move a stack a number of squares less than its size, and also may split a stack, moving only the top $n$ pieces. In this case, the split stack may only be moved up to $n$ squares. So, for example, if a player controlled a stack that was four pieces high, he could move the stack one to four squares up, down, left, or right, or could take the top two pieces only and move them up to two squares in the same four directions. However, he could not move the top two pieces three squares to the left, for example. 
If a move places a stack on top of another stack, the stacks are merged, where the moved pieces are placed on top of the other stack. If the newly created, merged stack has a size of $n>5$, then the bottom $n-5$ pieces are considered to be "captured" by the player who made the move, and are removed from the board. If a player captures his own piece, this opens up a new kind of move, where the player can place the captured piece back onto the board, on top of any stack, instead of moving a stack. To clarify how moves are made in Focus, a number of possible moves are shown in Figure A.6.
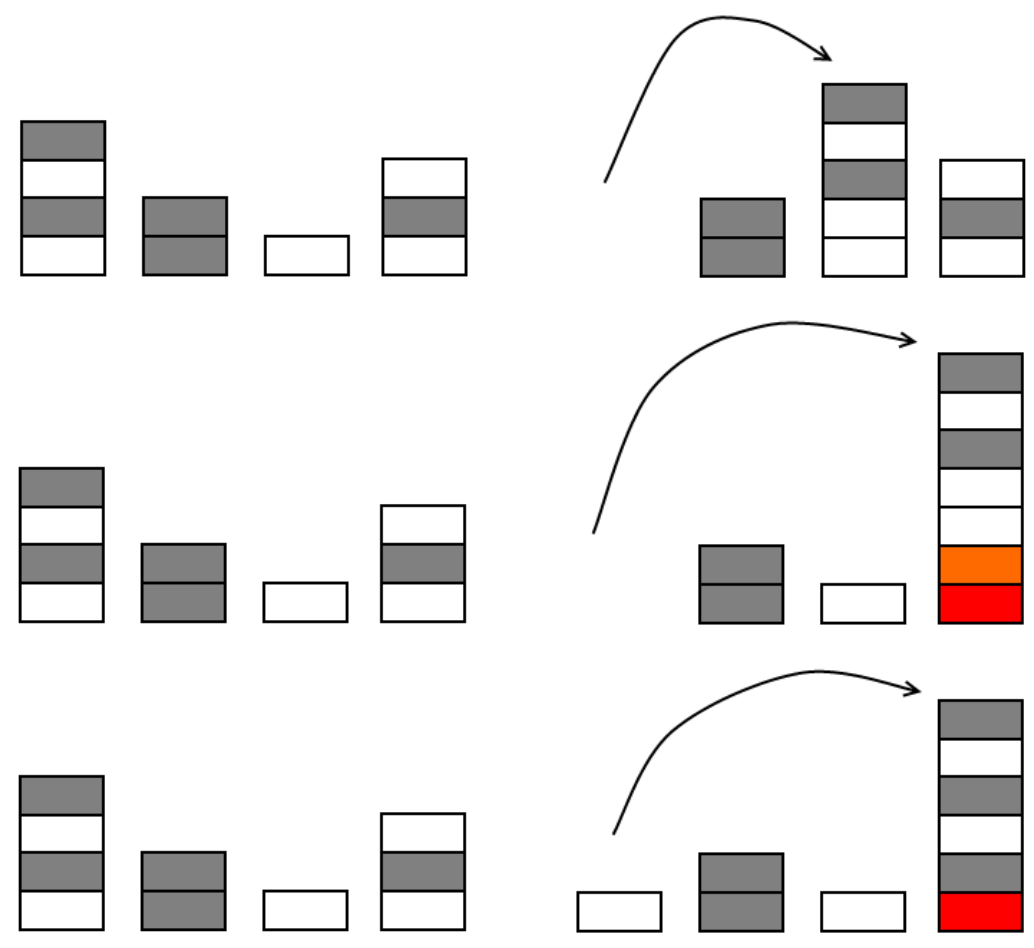

Figure A.6: Possible moves for the grey player in Focus, playing against white. Captured enemy pieces are coloured red, and the player's own captured pieces are coloured orange. 
Focus usually ends when only one player is capable of making a valid move, in which case he is declared to be the Winner. However, games with this termination criterion can take a very long time to complete, and therefore an alternative winning condition, due to Schadd and Winands, has also been specified in [52]. Under this winning condition, in the two-player version of the game, the player is declared to be the Winner if he has captured six pieces from the opponent. In the three-player case, a player is reckoned to have won if he has captured ten pieces in total, or three from each opponent. Finally, in the four player case, the player is said to have won if he has captured ten pieces in total, or two from each opponent. Finally, if the game is not decided after 300 moves, it is declared a draw. We make use of this shortened version of the game in this work, however it should be noted that games of Focus never reach even close to the move limit, usually being decided within a tenth of that.

The presence of stack splitting, as well as the second type of move where a player places one of his own pieces back on the board, renders Focus a tactically complex game. It, furthermore, has an extremely large branching factor. Consider, for instance, the situation where a player controls a stack of four pieces, near the center of the board. The entire stack could be moved to a total of 16 different positions (four steps in each direction, orthogonally), and besides these, the top three moves could be moved to a total of 12 different positions, the top two to 8 , and the very top piece alone, to 4 different positions (immediately adjacent to the stack). While some of these positions will be out of bounds in some configurations and thus be invalid, this single stack alone presents a total of nearly forty moves to the player, and multiple stacks may be present on the board for the player to choose from.

Furthermore, if a player has captured one of his own pieces, he may put it on any stack on the board instead of moving a stack, adding 52 possible moves of a completely different type. Thus, it is easy to see how a player may have well over a hundred possible moves available to him at times. This massive branching factor and complex state space must be accounted for in the game's implementation. We emphasize that a substantial effort was expended during the course of this research, to ensure that our Focus engine could run quickly enough to serve as a proper testing environment. 
Board Evaluation for Focus: The board evaluation function for Focus, that we will make use of in this work, is the same as the one that was introduced in [52], and is specified with special consideration for the shortened form of the game. We have chosen this approach to render our work to be as consistent as possible with the original testing environment of the BRS, specified in [52]. The board evaluation function consists of two terms. The first is the number of pieces, at minimum, that a player requires to win, under either of the win conditions (the minimum total, or the number from each player). This value is inverted, through subtraction from a sufficiently large number (ten is the maximum, so this is the number used), and multiplied by 1000, thus insuring that moves that take the player closer to a win are favoured. The second term favours having pieces located high in a stack, as these pieces are hard to capture, and may represent control of stacks, which is particularly valuable if the stack is tall. This term is equal to the sum, for each piece the player controls, of its height squared, which is added to the previous term. Finally, as Focus has a static starting position, unlike the Virus Game, a random factor of five is added to the evaluation function, to prevent repetitious play.

\section{A.4 Chinese Checkers}

Chinese Checkers is a well-known board game for two to four, or six players (five is excepted), first developed in 1893 and reprinted many times since [29]. Due to its relatively simple rules and large branching factor, as well as its popularity, as with Focus, it has seen use as a testing environment for game playing techniques and related research $[52,64]$.

Rules of Chinese Checkers: Chinese Checkers is played on a star shaped board, rather than a normal grid-based checkerboard, where each position is adjacent to six others, in a hexagon around it. Under normal circumstances, the board contains 121 positions, with ten on each of the "points" of the star [29]. However, this board size leads to a very large branching factor in the game tree, and therefore, a smaller 
version, with 73 positions total and six on each of the points is more commonly used in the literature $[52,64]$. We have chosen to employ this version in our work. Each of the players starts with enough pieces to fill one of the corners of the star, which is assigned to them based on the number of players in total, so in the smaller version of the game each player begins play with six pieces. Starting board arrangements for two, three, four, and six players are shown in Figure A.7. It is clear to see from this diagram, that the game cannot be played evenly with five players, as one player would not have an opponent across from him, and would thus have a significant advantage.
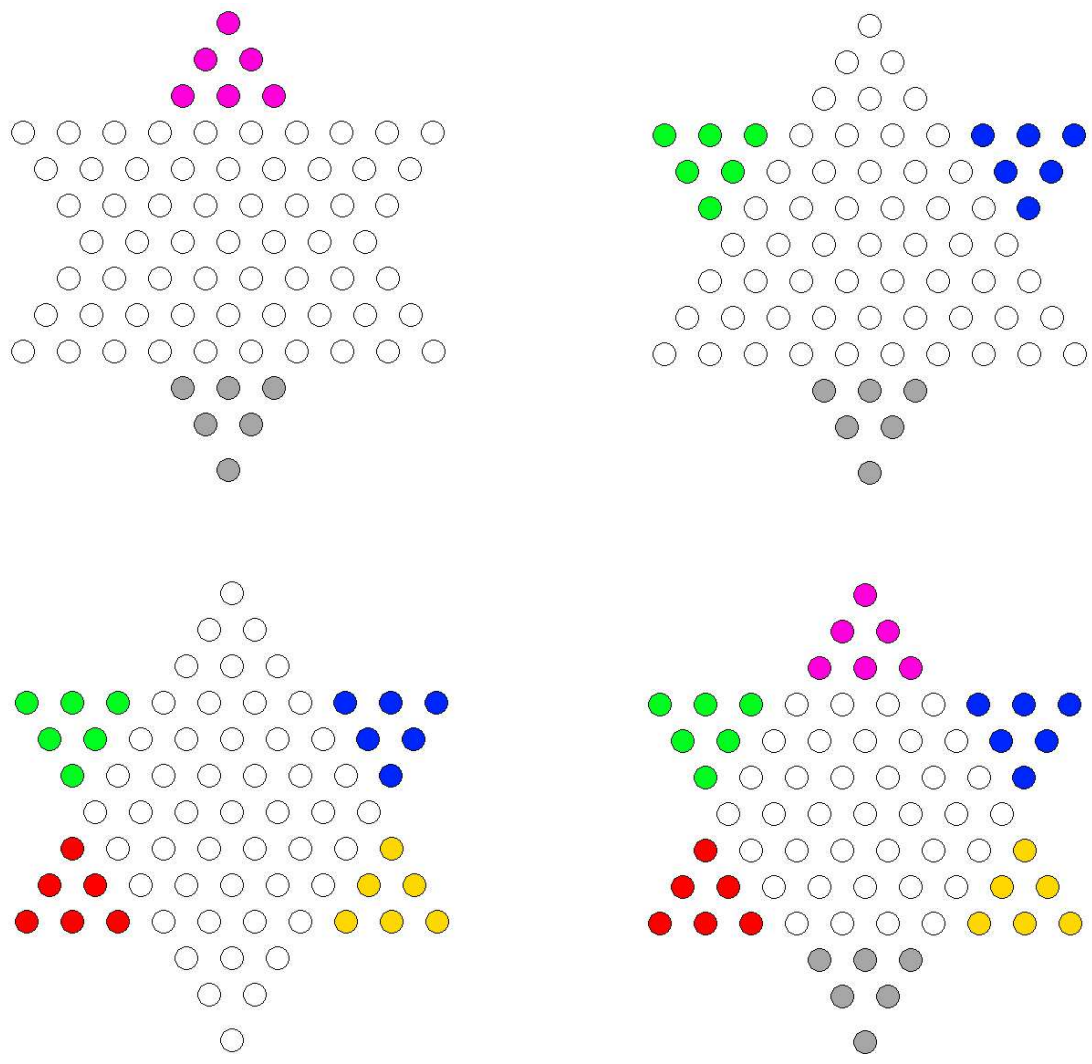

Figure A.7: The two, three, four, and six player starting positions for Chinese Checkers. 
During a player's turn, he may move any one his pieces to an unoccupied position adjacent to its current position, after which play passes to the next player. We will refer to this as a "normal" move. However, if an adjacent position is occupied, but the position adjacent to it in the same direction is not occupied, the player may make a "jumping" move, moving his piece directly to that unoccupied position, hopping over the adjacent piece. From here, as part of the same move, the player is permitted to make another jumping move with the same piece, if one is available, and to continue doing so until he chooses to stop. The player may not follow a series of jumping moves with a normal move. An example of a chain of jumping moves is shown in Figure A.8.

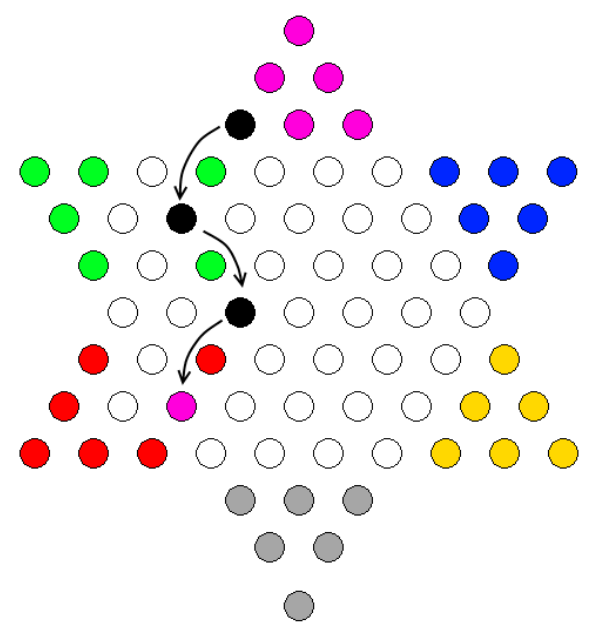

Figure A.8: A series of jumping moves by the purple player. The position the piece started at and passed through are coloured black.

Play continues until one player has managed to move all his pieces into the point on the star opposite from where he began, at which point that player is declared to be the Winner. Under some versions of the game, the remaining players will continue to play to decide the second, third, and so on ranks [29]. However, our work using Chinese Checkers as a game model is not as concerned with such a winning criterion, so this phase of the game is omitted. 
Even in its smaller form, Chinese Checkers possesses an appreciably large branching factor. Consider briefly, if the player's six pieces are spread about the center of the board. As each space in Chinese Checkers is adjacent to six others, and pieces can move in any direction, 36 different, single step moves are available to the player. Furthermore, as jumps can be chained, there may be many more available possibilities for a single piece to move around the board, in a variety of ways. Furthermore, unlike Focus, where, despite a massive branching factor, certain moves, i.e. moving large stacks to capture many pieces, are obviously strong, outside of the metric that the player should move their pieces towards the goal state, many intuitively "equal" moves exist in the context of Chinese Checkers.

The implementation of Chinese Checkers is complicated by the star-shaped board, as the board cannot be directly represented in memory using a simple, two-dimensional array. However, due to the large branching factor and strategic qualities of the game, employing a more complex, although easier to work with, data structure is not acceptable due to its impact on the game engine's runtime. Thus, in our Chinese Checkers implementation, we constructed a mapping of valid moves, to allow a two-dimensional array of an uneven size to be employed instead of resorting to a graph. Although this required a substantial refinement and testing process to insure accuracy to the rules of the game, it was necessary to be able to use Chinese Checkers as a testing environment.

Board Evaluation for Chinese Checkers: A number of board evaluation heuristics exist for Chinese Checkers, of varying complexity. The one, which is perhaps the best recorded, is the use of a lookup table, similar to a Transposition Table, that only factors in the player's own pieces, and that contains the minimum number of moves needed to win the game [64]. Clearly, factoring in only the player's pieces will lead to an erroneous assessment of the game state, particularly during the middle of the game. However, as this technique allows for perfect play in the end phases of the game, it usually outperforms other, simpler heuristics [52, 64]. Of course, this board evaluation function must be inverted, so that positions where a smaller number of moves are needed to win the game are more strongly favoured. 
A secondary board evaluation function, that is useful when setting up the perfect play table is problematic or too time consuming, is to take the sum of the distances, within the board, of each player's piece to the goal state. This evaluation function is analogous to the lookup table technique, if only single step moves were allowed within the game. Certainly, as it factors in less information, this evaluation function will be outperformed by the lookup table, although it does not require the storage and construction of a large table. Thus, given that our work is interested in measuring the $\mathrm{NC}$, rather than the absolute win-rate, we have chosen to employ this evaluation strategy, as opposed to the lookup table.

\section{A.5 Relaxed Checkers}

The game Checkers, also called Draughts, is a particularly well-known, and extensively studied two-player board game, that is widely played competitively. Although, recently, a mathematical solution for perfect Checkers play was presented [55], given the game's importance in historical game playing literature, and that it is a game with very familiar rules, we consider it to still have a great deal of utility as a testing environment for general purpose game playing strategies. Furthermore, while this makes the game less interesting from the perspective of a player, we shall make modifications to the game of Checkers so as to increase its state space and branching factor, as detailed presently.

Rules of Relaxed Checkers: The rules for Checkers are particularly well known, and will only be detailed here briefly for the sake of completeness. Checkers is played on an 8-by-8 checkerboard, between two players. Each player begins the game with 12 pieces, which are arranged on the top and bottom three rows, for each player, respectively, on the dark coloured squares on the board. The light coloured squares are not used in the game. The starting position for Checkers is shown in Figure A.9. 


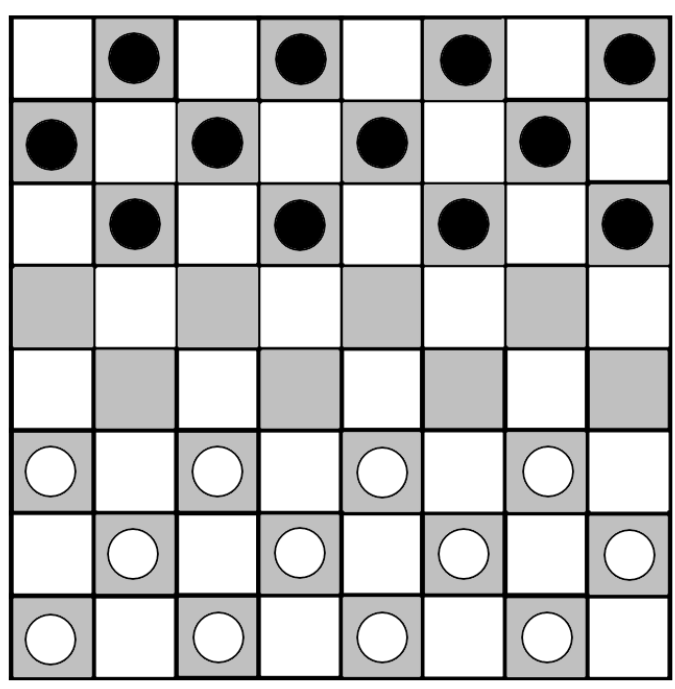

Figure A.9: The starting position for the game Checkers.

During a player's turn, he may move a single piece, diagonally, one position forward, to an unoccupied square. Thus, a single piece in Checkers will typically have only two possible moves available to it at any given time. Alternatively, if a piece is adjacent to an opponent's piece, and an unoccupied space exists across from it, the player can, instead, "jump" that piece, moving directly to said unoccupied space, as in the case of Chinese Checkers. The "jumped" piece is considered captured, and is removed from play. Furthermore, as in Chinese Checkers, jumping moves may be chained together, capturing multiple pieces in a single turn and advancing one's piece towards the opposite side of the board.

When a player's piece reaches the opposite side of the board, and cannot move forward anymore, it is promoted to be a "King" piece, and may thereafter move backwards and forwards freely, along the diagonals, and make capturing moves in either direction as well. When a player cannot make a move in Checkers, he is considered to have lost, and the other player is declared the Winner. This can be achieved by either capturing all of the opponent's pieces, or by placing them in a position where they cannot make a legal move on his turn. 
A major rule in Checkers is that not only may a player make a capturing jump if one is presented, but, in fact, he is required to do so. This rule is necessary for the strategic nature of the game, as a player can force the opponent to make a disadvantageous capturing move, setting the opponent up for a devastating response. However, in this work, we are interested in tree pruning, and not in creating a competitive Checkers player. Thus the larger branching factor afforded by relaxing this rule is advantageous to our research goals. Consequentially, we do not require players to make capturing moves, and if a sequence of jumps is presented to a player, he may choose to take only some of them. We refer to this new version of the game, as "Relaxed Checkers".

Even with the relaxation of the "mandatory capture" rule, Relaxed Checkers generally presents a smaller branching factor than both Focus and Chinese Checkers, and this is useful to us in the context of testing the performance of our strategies in trees with a greater ply depth. Relaxed Checkers did not present the same implementation difficulties that Focus and Chinese Checkers did. However the fact that players may choose to make only some capturing moves in a sequence required a refined move generation system, to avoid excessive computations in checking all the possibilities. Furthermore, in a normal Checkers engine, if capturing moves exist, one does not need to even generate non-capturing moves. As opposed to this, Relaxed Checkers did not afford us this luxury.

Board Evaluation for Relaxed Checkers: Given its extensive history in game playing literature, Checkers engines typically made use of highly refined board evaluation strategies, considering dozens of weighted factors, before the formal solution to Checkers was presented [55]. Among these factors include the number of pieces, the number of Kings, whether King pieces are trapped or able to move freely around the board, and a range of circumstantial variables within the game. However, we are more interested in using Checkers as a testing environment, rather than creating a highly refined, hand-tuned competitive strategy for the game ${ }^{1}$, and so certain forms of expert knowledge may not be applicable.

\footnotetext{
${ }^{1}$ Indeed, given it has been solved, such an endeavour would be nearly pointless, save as an exercise.
} 
Based on the above, we will only consider the highest weighted factors in our Relaxed Checkers evaluation, specifically, the number of total pieces, and the number of Kings, if the Kings are not trapped and unable to move about the board freely. The evaluation heuristic awards each player a point for each piece they control, and another half point per King piece, if the King is not trapped. The final value of the game state, for a given player, is its score, minus his opponents' score under the same heuristic.

\section{A.6 Othello}

Othello, also called Reversi, is a popular two-player board game, with an extensive history in the field of AI game playing, including, in its multi-player version, in the context of the BRS [52]. Othello lacks the complexity of Focus or of Chinese Checkers. Nevertheless, it has a number of unique qualities that set it aside from these games, including how pieces are captured, that make it useful as our final testing environment.

Rules of Othello: The version of Othello that we are concerned with, and is the most common commercial version, is played on an 8-by-8 board, similar to Focus and Checkers. Unlike in either of those games, however, the entire board is available to the players, although other restrictions are placed on available moves. The game's starting position is fixed, with the four central squares being occupied by the two of each player's pieces, alternating in colour in a clockwise fashion. To clarify matters, the starting position for Othello is shown in Figure A.10.

Play in Othello always begins with the black player. During his turn, a player may place a piece anywhere on the board, such that one or more of the opponents' pieces are "flanked" by this new piece, and one of his existing pieces on the board. This causes each of the flanked pieces to be captured and replaced by the player's pieces, or "flipped" in common parlance, given that Othello pieces are usually two-sided. Pieces cannot be placed on the board in isolation in Othello, and may only be played in this flanking position. An example of a valid Othello move for white is shown in 


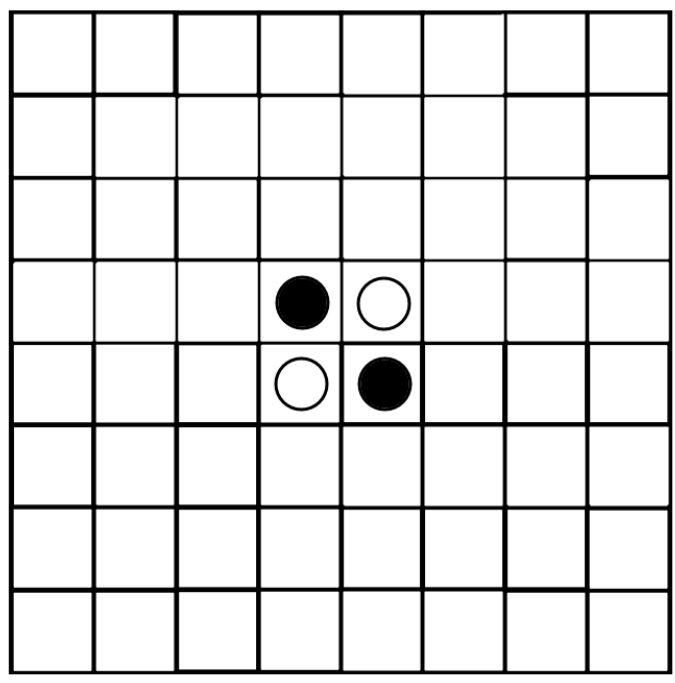

Figure A.10: The starting position for the game Othello.

Figure A.11. It is possible to place a piece in such a manner that two flanking lines are formed, in which case all the opponents' pieces along both these lines are flipped.

Unlike in Checkers or Chess, a player neither loses nor causes the end of the game if he is unable to make a valid move in Othello. Rather, he skips his turn, and the opponent is able to move again. The game ends when neither player can make a valid move, at which point the player with the larger number of pieces on the board, is declared the Winner.

Othello was comparatively the easiest game engine to implement for our purposes, given its relatively simple rules, square board, and the fact that moves do not "chain" together, as jumps do in the context of Relaxed Checkers or Chinese Checkers. Furthermore, given its restrictive number of available moves, Othello has a much smaller branching factor, and thus did not require as highly refined of a game engine as, say, Focus. However, this smaller branching factor allowed us to test the History-ADS heuristic in deeper Othello trees, which proved to be useful to our work. 

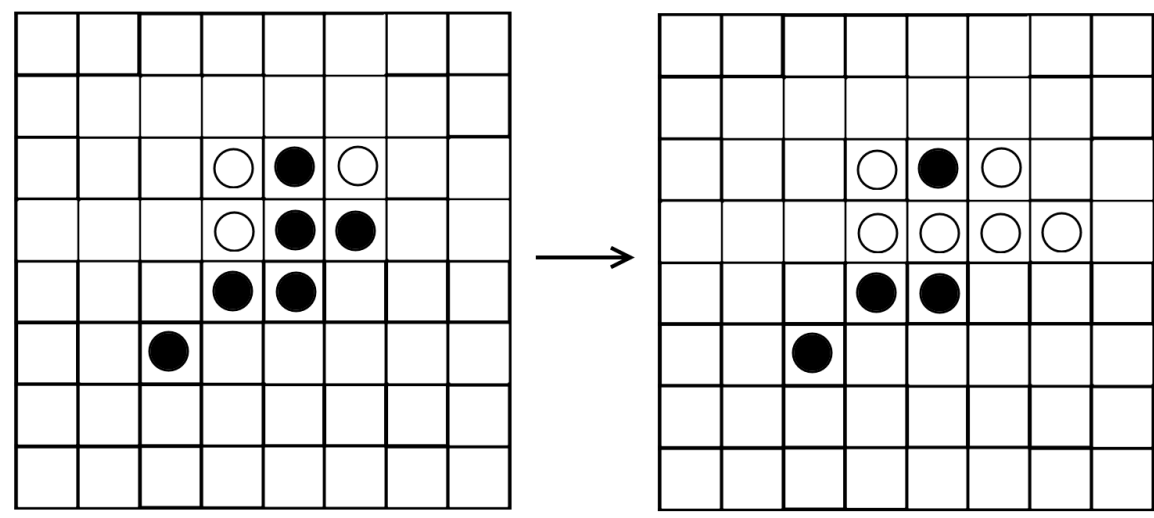

Figure A.11: An example of a valid move in Othello.

Board Evaluation for Othello: Competitive board evaluation functions for Othello, and its variants, are pattern based, employing a large database of pre-existing games, in order to determine the best possible move to make at each stage [52]. However, given the objectives in this research, we did not consider it to be an efficient use of our time, and thus decided to implement the simple evaluation heuristic of the number of pieces a player controls, minus the number his opponent controls, which proved sufficient for our purposes.

\section{A.7 Summary}

This appendix to the thesis has described, in detail, the games employed in our work, and the challenges faced in implementing their engines. Specifically, this involved the Virus Game, Focus, Chinese Checkers, Relaxed Checkers, and Othello. It has also specified the board evaluation functions that we employed in our work, for maximum transparency, and for the benefit of those attempting to replicate our work. 


\section{Bibliography}

[1] C. Adami, C. T. Brown, and W. K. Kellogg. Evolutionary learning in the $2 \mathrm{~d}$ artificial life system "avida". In Artificial Life IV, pages 377-381. MIT Press, 1994.

[2] S. Albers and J. Westbrook. Self-organizing data structures. In Online Algorithms, pages 13-51, 1998.

[3] B. Allen and I. Munro. Self-organizing binary search trees. Journal of the ACM, 25, 1978.

[4] G. M. Baudet. An analysis of the full alpha-beta pruning algorithm. In Proceedings of the tenth annual ACM symposium on Theory of computing, pages 296-313, 1978.

[5] J. Best. Ibm watson: How the jeopardy-winning supercomputer was born, and what it wants to do next. Contemporary article on Watson's victory in Jeopardy, 2013.

[6] R. Borie, C. Tovey, and S. Koenig. Algorithms and complexity results for pursuitevasion problems. In Proceedings of the Twenty-First International Joint Conference on Artificial Intelligence, pages 59-66, 2009.

[7] D. M. Breuker, J. W. H. M. Uiterwijk, and H. J. Van Den Herik. Replacement schemes for transposition tables. ICCA Journal, 17:183-193, 1994. 
[8] M. Buro. Probcut: An effective selective extension of the alphabeta algorithm. ICCA Journal, 18:71-76, 1995.

[9] M. S. Campbell and T. A. Marsland. A comparison of minimax tree search algorithms. Artificial Intelligence, pages 347-367, 1983.

[10] R. P. Cheetham, B. J. Oommen, and D. T. H. Ng. Adaptive structuring of binary search trees using conditional rotations. In Knowledge and Data Engineering, IEEE Transactions on, 1993.

[11] R. Coe. It's the effect size, stupid: What effect size is and why it is important. In Annual Conference of the British Educational Research Association, University of Exeter, Exeter, Devon, 2002.

[12] A. Cook and W. Edmondson. Using chunking to optimise an alpha-beta search. In Technologies and Applications of Artificial Intelligence (TAAI), 2010 International Conference on, pages 166-171, 2010.

[13] T. H. Corman, C. E. Leiserson, R. L. Rivest, and C. Stein. Introduction to Algorithms, pages 302-320. MIT Press, Upper Saddle River, NJ, USA, 3rd edition, 2009.

[14] E. D. Demaine, D. Harmon, J. Iacono, and M. Patrascu. Dynamic optimalityalmost. In Special issue of selected papers from the 45th Annual IEEE Symposium on Foundations of Computer Science., 2007.

[15] R. P. Dobrow and J. A. Fill. Rates of convergence for a self-organizing scheme for binary search trees. In Annals of Applied Probability, 1994.

[16] M. Esser, M. Gras, M. H. M. Winands, M. P. D. Schadd, and M. Lanctot. Improving best-reply search. In Proceedings of CG'2013 the Eighth International Conference on Computers and Games, pages 125-137, 2014.

[17] V. Estivill-Castro. Move-To-End is best for double-linked lists. In Proceedings of the Fourth International Conference on Computing and Information, pages 84-87, 1992. 
[18] D. Fudenberg and J. Tirole. Game Theory. MIT Press, 1991.

[19] S. Gelly and Y. Wang. Exploration Exploitation in Go: UCT for Monte-Carlo Go. In Proceedings of NIPS'06, the 2006 Annual Conference on Neural Information Processing Systems, 2006.

[20] G. H. Gonnet, J. I. Munro, and H. Suwanda. Towards self-organizing linear search. In Proceedings of FOCS'r9, the 1979 Annual Symposium on Foundations of Computer Science, pages 169-171, 1979.

[21] J. H. Hester and D. S. Hirschberg. Self-organizing linear search. ACM Computing Surveys, 17:285-311, 1985.

[22] J. Huang, Z. Liu, L. Benjie, and X. Feng. Pruning in uct algorithm. In Proceedings of TAAI'10, the 2010 International conference on Technologies and Applications of Artificial Intelligence, pages 177-181, 2010.

[23] C. A. Kirts, M. A. Tumeo, and J. M. Sinz. The commons game: its instructional value when used in a natural resources management context. Simul. Gaming, pages $5-18,1991$.

[24] A. Kishimoto and J. Schaeffer. Distributed game-tree search using transposition table driven work scheduling. In Parallel Processing, 2002. Proceedings. International Conference on, pages 323-330, 2002.

[25] D. E. Knuth and R. W. Moore. An analysis of alpha-beta pruning. Artificial Intelligence, 6:293-326, 1975.

[26] L. Kocsis and C. Szepesvari. Bandit based monte-carlo planning. In Proceedings of ECML'06, the 2006 European Conference on Machine Learning, pages 282293, 2006.

[27] R. E. Korf. Multiplayer alpha-beta pruning. In Artificial Intelligence, vol. 48 no. 1, 1991. 
[28] C. Luckhardt and K. Irani. An algorithmic solution of n-person games. In Proceedings of the AAAI'86, pages 158-162, 1986.

[29] M. S. Mohr. The New Games Treasury. Mariner Books, 1997.

[30] C. Moyer. How google's alphago beat a go world champion. The Atlantic, 2016.

[31] T Onisawa and T Yono. Construction of poker playing system considering strategies. In Proceedings of CyberGames'06, the 2006 International Conference on Game Research and Development, pages 121-128, 2006.

[32] B. J. Oommen and E. R. Hansen. Expedient stochastic move-to-front and optimal stochastic move-to-rear list organizing strategies. In Lecture Notes in Computer Science, 1986.

[33] B. J. Oommen and D. C. Y. Ma. Deterministic learning automata solutions to the equipartitioning problem. IEEE Transactions on Computers, 37, 1988.

[34] B. J. Oommen and D. T. H. Ng. Optimal constant space move-to-rear list organization. In Lecture Notes in Computer Science, 1989.

[35] S. Pettie. Splay trees, davenport-schinzel sequences, and the deque conjecture. In Proceedings of the nineteenth annual ACM-SIAM symposium on Discrete algorithms, 2008.

[36] A. Plaat, J. Schaeffer, W. Pijls, and A. de Bruin. A minimax algorithm better than alpha-beta? no and yes. Technical report, Department of Computer Science, University of Alberta, 1995.

[37] S. Polk and B. J. Oommen. On applying adaptive data structures to multi-player game playing. In Proceedings of AI'2013, the Thirty-Third SGAI Conference on Artificial Intelligence, pages 125-138, 2013.

[38] S. Polk and B. J. Oommen. On enhancing recent multi-player game playing strategies using a spectrum of adaptive data structures. In In Proceedings of TAAI'2013, the 2013 Conference on Technologies and Applications of Artificial Intelligence, pages 164-169, 2013. 
[39] S. Polk and B. J. Oommen. Enhancing history-based move ordering in game playing using adaptive data structures. In Proceedings of ICCCI'2015, the 7th International Conference on Computational Collective Intelligence Technologies and Applications, pages 225-235, 2015.

[40] S. Polk and B. J. Oommen. Novel AI strategies for multi-player games at intermediate board states. In Proceedings of IEA/AIE'2015, the Twenty-Eighth International Conference on Industrial, Engineering, and Other Applications of Applied Intelligent Systems, pages 33-42, 2015.

[41] S. Polk and B. J. Oommen. Space and depth-related enhancements of the historyads strategy in game playing. In In Proceedings of CIG'2015, the 2015 IEEE Conference on Computational Intelligence and Games, pages 322-327, 2015.

[42] S. Polk and B. J. Oommen. Challenging established move ordering strategies with adaptive data structures. In Proceedings of IEA/AIE'2016, the Twenty-Ninth International Conference on Industrial, Engineering, and Other Applications of Applied Intelligent Systems, 2016.

[43] S. Polk and B. J. Oommen. Novel threat-based ai strategies for multi-player games. In Submitted for Publication, 2016.

[44] S. Polk and B. J. Oommen. On achieving history-based move ordering in adversarial board games using adaptive data structures. In Transactions on Computational Collective Intelligence XXII, pages 10-44, 2016.

[45] A. Reinefeld and T. A. Marsland. Enhanced iterative-deepening search. IEEE Transactions on Pattern Analysis and Machine Intelligence, 16:701-710, 1994.

[46] A. Rimmel, O. Teytaud, C. Lee, S. Yen, M. Wang, and S. Tsai. Current frontiers in computer go. IEEE Transactions on Computational Intelligence and Artificial Intelligence in Games, page in press, 2010.

[47] R. L. Rivest. On self-organizing sequential search heuristics. In Proceedings of the 1974 IEEE Symposium on Switching and Automata Theory, pages 63-67, 1974. 
[48] S. J. Russell and P. Norvig. Artificial Intelligence: A Modern Approach. PrenticeHall, Inc., Upper Saddle River, NJ, USA, 3rd edition, 2009.

[49] S. J. Russell and P. Norvig. Artificial Intelligence: A Modern Approach, pages 161-201. Prentice-Hall, Inc., Upper Saddle River, NJ, USA, 3rd edition, 2009.

[50] S. Sacksin. A Gamut of Games. Random House, 1969.

[51] M. P. D. Schadd and M. H. M. Winands. Quiescence search for stratego. In BNAIC 2009 T. Calders, K. Tuyls, M. Pechenizkiy, eds., pages 225-232, 2009.

[52] M. P. D. Schadd and M. H. M. Winands. Best Reply Search for multiplayer games. IEEE Transactions on Computational Intelligence and AI in Games, 3:57-66, 2011.

[53] M. P. D. Schadd, M. H. M. Winands, and J. W. H. M. Uiterwijk. Chanceprobcut: forward pruning in chance nodes. In Proceedings of the 5th international conference on Computational Intelligence and Games, pages 178-185, 2009.

[54] J Schaeffer. The history heuristic and alpha-beta search enhancements in practice. IEEE Transactions on Pattern Analysis and Machine Intelligence, 11:12031212, 1989.

[55] J. Schaeffer, N. Burch, Y. Bjornsson, A. Kishimoto, M. Muller, R. Lake, P. Lu, and S. Sutphen. Checkers is solved. Science, 14:1518-1522, 2007.

[56] S. Schaffer. Enlightened automata. In The Sciences in Enlightened Europe, pages 126-164. University of Chicago Press, 1999.

[57] E. Schrder. Move ordering in rebel. Discussion of move ordering techniques used in REBEL, a powerful chess engine, 2007.

[58] C. E. Shannon. Programming a computer for playing Chess. Philosophical Magazine, 41:256-275, 1950.

[59] A. Silver. Top 10 man-vs.-machine moments. Time Magazine, 2011. 
[60] B. Skyrms. The stag hunt and the evolution of social structure. Cambridge University Press, 2004.

[61] D. D. Sleator and R. E. Tarjan. Amortized efficiency of list update and paging rules. Communications of the ACM, 28:202-208, 1985.

[62] N. Sturtevant. A comparison of algorithms for multi-player games. In Proceedings of the Third International Conference on Computers and Games, pages 108-122, 2002.

[63] N. Sturtevant. Multi-Player Games: Algorithms and Approaches. PhD thesis, University of California, 2003.

[64] N. Sturtevant. An analysis of uct in multi-player games. In In Computers and Games, 2008.

[65] N. Sturtevant and M. Bowling. Robust game play against unknown opponents. In Proceedings of AAMAS'06, the 2006 International Joint Conference on Autonomous Agents and Multiagent Systems, pages 713-719, 2006.

[66] N. Sturtevant, M. Zinkevich, and M. Bowling. Prob-Maxn: Playing n-player games with opponent models. In Proceedings of AAAI'06, the 2006 National Conference on Artificial Intelligence, pages 1057-1063, 2006.

[67] I. Szita, G. Chaslot, and P. Spronck. Monte-carlo tree search in settlers of catan. In Advances in Computer Games: 12th International Conference, ACG 2009, pages 21-32, 2010.

[68] I. Szita, C. Guillame, and P. Spronck. Monte-carlo tree search in settlers of catan. In Proceedings of ACG'09, the 2009 Conference on Advances in Computer Games, pages 21-32, 2009.

[69] A. Turner and J Miller. The importance of topology evolution in neuroevolution: A case study using cartesian genetic programming of artificial neural networks. In In Proceedings of AI'2013, the Thirty-Third SGAI Conference on Artificial Intelligence, pages 213-226, 2013. 
[70] C. C. Wang, J. Derryberry, and D. D. Sleator. O(log $\log n)$-competitive dynamic binary search trees. In Proceedings of the seventeenth annual ACM-SIAM symposium on Discrete algorithm, 2006.

[71] Y. Wang and S. Gelly. Modifications of uct and sequence-like simulations for monte-carlo go. In Computational Intelligence and Games, 200\%. CIG 200\%. IEEE Symposium on, pages 175-182, 2007.

[72] F. Xiao and Z Liu. Modification of uct algorithm with quiescent search in computer go. In Proceedings of TAAI'10, the 2010 International Conference on Technologies and Applications of Artificial Intelligence, pages 481-484, 2010.

[73] J. Zhang, X. Wang, J. Lin, and Z. Xu. Uct algorithm in imperfect information multi-player military Chess game. In Proceedings of the 11th Joint Conference on Information Sciences, 2008.

[74] I. Zuckerman. The mp-mix algorithm: Dynamic search strategy selection in multiplayer adversarial search. IEEE Transactions on Computational Intelligence and AI in Games, 3, 2011.

[75] I. Zuckerman, A. Felner, and S. Kraus. Mixing search strategies for multi-player games. In Proceedings of IJCAI'09, the Twenty-first International Joint Conferences on Artificial Intelligence, pages 646-651, 2009. 